\title{
Rifamycin Biosynthetic Congeners: Isolation and Total Synthesis of Rifsaliniketal, and Total Synthesis of Salinisporamycin and Saliniketals A and B
}

\author{
Yu Feng, ${ }^{\dagger}$ Jun Liu, ${ }^{\dagger}$ Yazmin P. Carrasco, ${ }^{\dagger}$ John B. MacMillan, ${ }^{*, \dagger}$ and Jef K. De Brabander ${ }^{*, \dagger, \ddagger}$ \\ ${ }^{\dagger}$ Department of Biochemistry and ${ }^{\ddagger}$ Harold C. Simmons Comprehensive Cancer Center, The University of Texas Southwestern \\ Medical Center at Dallas, 5323 Harry Hines Boulevard, Dallas, Texas 75390-9038
}

\section{SUPPORTING INFORMATION}

\section{Contents}

1. General Experimental...................................................................

2. Isolation and Characterization of Rifsaliniketal .....................................S2

3. Experimental Procedures...............................................................S6

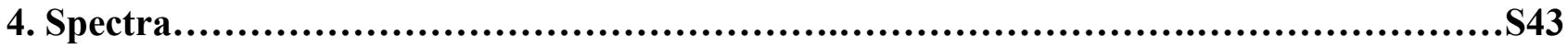




\section{General Experimental}

Unless otherwise noted, commercially available materials were used without further purification.

Solvents used for moisture sensitive operations were tapped from a solvent purification system immediately before use. Reactions were performed under an atmosphere of nitrogen with magnetic stirring unless noted otherwise. Flash chromatography (FC) was performed using E. Merck silica gel 60 (240-400 mesh). Thin layer chromatography was performed using precoated plates purchased from $E$. Merck (silica gel $60 \mathrm{PF}_{254}, 0.25 \mathrm{~mm}$ ) that were visualized using a $\mathrm{KMnO}_{4}$ or $\mathrm{Ce}$ (IV) stain.

Nuclear magnetic resonance (NMR) spectra were recorded on a Varian Inova-400, Varian Inova-500 or Varian Inova-600 spectrometer at operating frequencies of 400/500/600 MHz ( ${ }^{1} \mathrm{H}$ NMR) or 100/125/150 $\mathrm{MHz}\left({ }^{13} \mathrm{C}\right.$ NMR). Chemical shifts $(\delta)$ are given in ppm relative to residual solvent (usually chloroform $\delta$ 7.26 for ${ }^{1} \mathrm{H}$ NMR or $\delta 77.16$ for proton decoupled ${ }^{13} \mathrm{C}$ NMR), and coupling constants $(J)$ in $\mathrm{Hz}$. Multiplicity is tabulated as s for singlet, $\mathrm{d}$ for doublet, $\mathrm{t}$ for triplet, $\mathrm{q}$ for quadruplet, and $\mathrm{m}$ for multiplet, whereby the prefix app is applied in cases where the true multiplicity is unresolved, and br when the signal in question is broadened.

Infrared spectra were recorded on a Perkin-Elmer I1000 series FTIR with wavenumbers expressed in $\mathrm{cm}^{-1}$ using samples prepared as thin films between salt plates. Electrospray ionization mass spectra (ESI-MS) were recorded on a Shimadzu 2010-LCMS. Optical rotations were measured at $20{ }^{\circ} \mathrm{C}$ on a Rudolph Research Analytical Autopol ${ }^{\circledR}$ IV polarimeter.

Procedures for the preparation of compounds $\mathbf{1 b}, \mathbf{1 5}, \mathbf{1 4 b}, \mathbf{2 8 - 3 0}$, 22-anti, 23-anti, 33, 39-41, and 42b-45b along with compound characterizations and copies of NMR spectra can be found in the Supporting Information associated with: Liu, J.; De Brabander, J. K. J. Am. Chem. Soc. 2009, 131, 12562-12563.

\section{Isolation and Characterization of Rifsaliniketal}

\section{Collection and Phylogenetic Analysis}

The marine-derived bacterium strain SNB-003, was isolated from sediment sample collected from Trinity

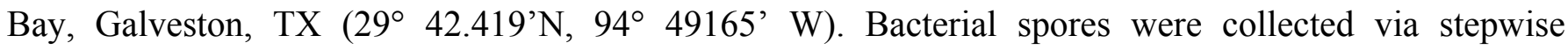
centrifugation as follows: $2 \mathrm{~g}$ of sediment was dried over $24 \mathrm{~h}$ in an incubator at $35^{\circ} \mathrm{C}$ and the resulting

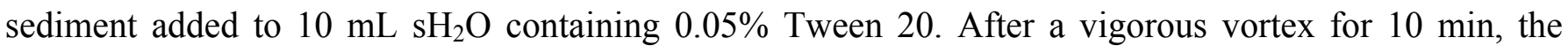
sediment was centrifuged at $18000 \mathrm{rpm}$ for $25 \mathrm{~min}\left(4^{\circ} \mathrm{C}\right)$ and the resulting spore pellet collected. The resuspended spore pellet ( $4 \mathrm{~mL} \mathrm{sH} 2 \mathrm{O}$ ) was plated on an acidified Gauze media, giving rise to individual colonies of SNB-003 after two weeks. Analysis of the 16S rRNA sequence of SNB-003 revealed $98 \%$ identity to Salinospora arenicola.

Cultivation and extraction: Bacterium SNB-003 was cultured in $120 \AA \sim 2.8 \mathrm{~L}$ Fernbach flasks each containing $1 \mathrm{~L}$ of a seawater based medium (10 g starch, $4 \mathrm{~g}$ yeast extract, $2 \mathrm{~g}$ peptone, $1 \mathrm{~g} \mathrm{CaCO}, 40 \mathrm{mg}$ 
$\left.\mathrm{Fe}_{2}\left(\mathrm{SO}_{4}\right)_{3} .4 \mathrm{H}_{2} \mathrm{O}, 100 \mathrm{mg} \mathrm{KBr}\right)$ and shaken at $200 \mathrm{rpm}$ at $27^{\circ} \mathrm{C}$. After seven days of cultivation, sterilized XAD-7-HP resin (20 g/L) was added to adsorb the organic products, and the culture and resin were shaken at $200 \mathrm{rpm}$ for $2 \mathrm{~h}$. The resin was filtered through cheesecloth, washed with deionized water, and eluted with acetone. The acetone soluble fraction was dried in vacuo to yield $22.1 \mathrm{~g}$ of extract.

Isolation of 9: The dried crude extract $(22.1 \mathrm{~g})$ was purified using solvent/solvent partitioning. The active methanol soluble portion (16.0 g) was further partitioned using EtOAc and $\mathrm{H}_{2} \mathrm{O}$ (1:1 mixture). The ethyl acetate layer $(2.2 \mathrm{~g})$ was purified via reversed phase flash column chromatography, eluting with a step gradient of $\mathrm{H}_{2} \mathrm{O}$ and $\mathrm{MeOH}$ (90:10 to 100:0) collecting 12 fractions. Fraction 12 (89.5 mg) was purified by reversed phase HPLC (Phenomenex Luna, Phenyl-Hexyl, $250 \AA \AA \sim 10 \mathrm{~mm}, 2.5 \mathrm{ml} / \mathrm{min}, 5 \mu \mathrm{m}$, UV = 254 $\mathrm{nm}$ ) using a gradient solvent system from $20 \%$ to $100 \% \mathrm{CH}_{3} \mathrm{CN}(+0.1 \%$ formic acid) over $20 \mathrm{~min}$, collecting 11 fractions. Fraction $4(8.7 \mathrm{mg})$ was further purified by reversed phase HPLC (Phenomenex Luna, Phenyl-Hexyl, $250 \AA ̊ 10 \mathrm{~mm}, 2.5 \mathrm{ml} / \mathrm{min}, 5 \mu \mathrm{m}, \mathrm{UV}=254 \mathrm{~nm}$ ), a gradient solvent system was utilized (10\% to $100 \% \mathrm{CH} 3 \mathrm{CN}+0.1 \%$ formic acid over $26 \mathrm{~min})$ to give $9(1.70 \mathrm{mg})$.

\section{Rifsaliniketal (9)}

Physical state: yellow solid

Optical Rotation: $[\alpha]_{D}^{20}=+30.4(c=0.5, \mathrm{MeOH})$

UV (MeOH) $\lambda \max (\log \varepsilon) 216$ (3.80), 281 (3.71), 338 (3.73), 433 (3.44)

${ }^{1}$ H NMR, ${ }^{13}$ C NMR, COSY, HMBC correlations: (table S1).

MS (ES): $m / z 664.2[\mathrm{M}+\mathrm{Na}]^{+}, 640.2[\mathrm{M}-\mathrm{H}]^{-}$

HR-ESIMS: $m / z$ 642.2901 $[\mathrm{M}+\mathrm{H}]^{+}\left(\mathrm{C}_{34} \mathrm{H}_{44} \mathrm{NO}_{11} \text {, calculated 642.2908); 664.2713 [M }+\mathrm{Na}\right]^{+}$ $\left(\mathrm{C}_{34} \mathrm{H}_{43} \mathrm{NO}_{11} \mathrm{Na}\right.$, calculated 664.2728)

Conversion of 9 to 9a: To a solution of $9(0.37 \mathrm{mg}, 0.57 \mu \mathrm{mol})$ dissolved in dry $\mathrm{MeOH}(300 \mu \mathrm{L})$ was added $\mathrm{TMSCHN}_{2}(0.6 \mu \mathrm{L})$. After stirring for $15 \mathrm{~min}$, the reaction was stopped and the mixture was dried under nitrogen and was purified by reverse phase HPLC (Phenomenex Luna, C18, $150 \mathrm{~mm} \AA \sim 4.6 \mathrm{~mm}, 5$ $\mu \mathrm{m})$ isocratic conditions were utilized $\left(58 \% \mathrm{CH}_{3} \mathrm{CN}+0.1 \%\right.$ formic acid over $\left.15 \mathrm{~min}\right)$ followed by a gradient system from $58 \%$ to $100 \% \mathrm{CH}_{3} \mathrm{CN}+0.1 \%$ formic acid over 8 min to give 9a $(0.20 \mathrm{mg}, 53 \%$ yield). Rotamers were present in the sample in an approximate ratio of 0.6:0.4.

\section{Rifsaliniketal methyl ester (9a)}

${ }^{1}$ H NMR (600 MHz, MeOH-d4); signals of the major rotamer: $\delta 7.44$ (s, 1H), 6.78 (dd, $J=11.2 \mathrm{~Hz}, 15.2$ $\mathrm{Hz}, 1 \mathrm{H}), 6.48(\mathrm{~d}, J=11.2,1 \mathrm{H}), 6.04(\mathrm{dd}, J=8.0 \mathrm{~Hz}, 15.2 \mathrm{~Hz}, 1 \mathrm{H}), 4.21(\mathrm{dd}, J=6.7 \mathrm{~Hz}, 3.4 \mathrm{~Hz}, 1 \mathrm{H}), 3.94$ (d, $J=10.5 \mathrm{~Hz}, 1 \mathrm{H}), 3.87$ (s, 3H), 3.79 (d, $J=9.1 \mathrm{~Hz}, 1 \mathrm{H}), 3.51(\mathrm{dd}, J=8.3 \mathrm{~Hz}, 4.3 \mathrm{~Hz}, 1 \mathrm{H}), 2.43(\mathrm{~m}, 1 \mathrm{H})$, $2.07(\mathrm{~s}, 3 \mathrm{H}), 2.01(\mathrm{~s}, 3 \mathrm{H}), 1.78-1.83(\mathrm{~m}, 1 \mathrm{H}), 1.83-1.86(\mathrm{~m}, 1 \mathrm{H}), 1.86-1.89(\mathrm{~m}, 1 \mathrm{H}), 1.89-1.93(\mathrm{~m}, 1 \mathrm{H})$, 
1.93-1.97 (m, 1H), 1.97-2.01(m, 1H), 2.01-2.05 (m, 1H), 1.39 (s, 3H), 1.01 (d, $J=7.3 \mathrm{~Hz}, 3 \mathrm{H}), 0.99$ (d, $J$ $=7.3 \mathrm{~Hz}, 3 \mathrm{H}), 0.89(\mathrm{~d}, J=7.1 \mathrm{~Hz}, 3 \mathrm{H}), 0.71(\mathrm{~d}, J=6.9 \mathrm{~Hz}, 3 \mathrm{H})$

MS (ES): $\mathrm{m} / \mathrm{z} 678.3[\mathrm{M}+\mathrm{Na}]^{+}, 654.2[\mathrm{M}-\mathrm{H}]^{-}$

Table S1. 1D and 2D NMR data of rifsaliniketal (9) in $\mathrm{CD}_{3} \mathrm{OD}(600 \mathrm{MHz})$

\begin{tabular}{|c|c|c|c|c|}
\hline No. & $\delta_{\mathrm{H}}$, mult. $(J$ in $\mathrm{Hz})$ & $\boldsymbol{\delta}_{\mathbf{C}}$ & COSY & НМВС \\
\hline 1 & & $\mathrm{a}$ & & \\
\hline 2 & & $141.1 \mathrm{qC}^{\mathrm{b}}$ & & 3 \\
\hline 3 & 7.66, s & $117.5 \mathrm{CH}^{\mathrm{b}}$ & & $2,4,10$ \\
\hline 4 & & $183.8 \mathrm{qC}^{\mathrm{b}}$ & & 3 \\
\hline 5 & & $117.5 \mathrm{qC}^{\mathrm{b}}$ & & 12 \\
\hline 6 & & $162.6 \mathrm{qC}^{\mathrm{b}}$ & & 12 \\
\hline 7 & & $a^{2}$ & & \\
\hline 8 & & a & & \\
\hline 9 & & a & & \\
\hline 10 & & $128.6 \mathrm{qC}^{\mathrm{b}}$ & & 3 \\
\hline 11 & & a & & \\
\hline 12 & $2.16, \mathrm{~s}$ & $7.9 \mathrm{CH}_{3}{ }^{\mathrm{b}}$ & & 5,6 \\
\hline 13 & & $169.7 \mathrm{qC}$ & & 15,29 \\
\hline 14 & & $129.3 \mathrm{qC}$ & & 16,29 \\
\hline 15 & $6.46, \mathrm{~d}(11.2)$ & $138.0 \mathrm{CH}$ & 16,29 & $13,16,17,29$ \\
\hline 16 & $6.78, \mathrm{dd}(11.2,15.2)$ & $127.3 \mathrm{CH}$ & 15,17 & $14,15,18$ \\
\hline 17 & $6.02, \mathrm{dd}(8.0,15.2)$ & $145.4 \mathrm{CH}$ & 16,18 & $15,18,19,30$ \\
\hline 18 & 2.42, ddq $(9.1,8.0,6.9)$ & $42.0 \mathrm{CH}$ & $17,19,30$ & $16,17,19,30$ \\
\hline 19 & $3.77, \mathrm{dd}(9.1,1.4)$ & $75.5 \mathrm{CH}$ & 18,20 & $17,18,20,21,30,31$ \\
\hline 20 & $1.86-1.89, \mathrm{~m}$ & $35.8 \mathrm{CH}$ & $19,21,31$ & 21,31 \\
\hline 21 & $3.51, \mathrm{dd}(8.3,4.3)$ & $77.9 \mathrm{CH}$ & 20,22 & 19,22 \\
\hline 22 & $1.83-1.86, \mathrm{~m}$ & $36.7 \mathrm{CH}$ & $21,23,32$ & 21,32 \\
\hline 23 & $3.95, \mathrm{dd}(10.6,1.1)$ & $74.7 \mathrm{CH}$ & 22,24 & $21,22,32,34$ \\
\hline 24 & $1.97-2.01, \mathrm{~m}$ & $35.0 \mathrm{CH}$ & $23,25,34$ & \\
\hline 25 & $4.22, \mathrm{dd}(6.7,3.4)$ & $81.3 \mathrm{CH}$ & 24,26 & 23,28 \\
\hline $26 a$ & $1.89-1.93, \mathrm{~m}$ & $24.6 \mathrm{CH}_{2}$ & 25 & $24,25,27$ \\
\hline $26 b$ & 1.93-1.97, m & & & \\
\hline $27 \mathbf{a}$ & $1.78-1.83, \mathrm{~m}$ & $34.9 \mathrm{CH}_{2}$ & & 26 \\
\hline $27 b$ & $2.01-2.05, \mathrm{~m}$ & & & \\
\hline 28 & & $106.2 \mathrm{qC}$ & & 25,34 \\
\hline 29 & $2.08, \mathrm{~s}$ & $20.3 \mathrm{CH}_{3}$ & 15 & $13,14,15$ \\
\hline 30 & $0.99, \mathrm{~d}(6.9)$ & $16.7 \mathrm{CH}_{3}$ & 18 & $17,18,19$ \\
\hline 31 & $1.01, \mathrm{~d}(7.2)$ & $10.9 \mathrm{CH}_{3}$ & 20 & $19,20,21$ \\
\hline 32 & $0.89, \mathrm{~d}(7.0)$ & $10.1 \mathrm{CH}_{3}$ & 22 & $21,22,23$ \\
\hline 33 & $1.39, \mathrm{~s}$ & $24.0 \mathrm{CH}_{3}$ & & 27,28 \\
\hline 34 & $0.73, \mathrm{~d}(6.9)$ & $12.6 \mathrm{CH}_{3}$ & 24 & $23,24,25$ \\
\hline
\end{tabular}

${ }^{a}$ Shifts not determined due to small amount of material. ${ }^{b}$ Shifts determined from HMBC. 
Table S2. ${ }^{1} \mathrm{H}$ and ${ }^{13} \mathrm{C}$ NMR for natural rifsaliniketal $(600 \mathrm{MHz})$ and natural salinisporamycin $(500 \mathrm{MHz})$ in $\mathrm{CD}_{3} \mathrm{OD}$

\begin{tabular}{|c|c|c|c|c|}
\hline \multicolumn{3}{|c|}{ Rifsaliniketal } & \multicolumn{2}{|l|}{ Salinisporamycin } \\
\hline No. & $\delta_{\mathrm{H}}$, mult. $(J$ in $\mathrm{Hz})$ & $\delta_{\mathrm{C}}$ & $\delta_{\mathrm{H}}$, mult. $(J$ in $\mathrm{Hz})$ & $\delta_{\mathrm{C}}$ \\
\hline 1 & & $\mathrm{a}$ & & 181.3 \\
\hline 2 & & $141.1^{\mathrm{b}}$ & & 143.2 \\
\hline 3 & $7.66, \mathrm{~s}$ & $117.5^{\mathrm{b}}$ & $7.55, \mathrm{~s}$ & 116.4 \\
\hline 4 & & $183.8^{\mathrm{b}}$ & & 187.9 \\
\hline 5 & & $117.5^{\mathrm{b}}$ & $6.96, \mathrm{~s}$ & 112.7 \\
\hline 6 & & $162.6^{\mathrm{b}}$ & & 164.5 \\
\hline 7 & & a & & 117.8 \\
\hline 8 & & a & & 172.0 \\
\hline 9 & & a & & 106.6 \\
\hline 10 & & $128.6^{\mathrm{b}}$ & & 132.5 \\
\hline 11 & & a & $\mathrm{c}$ & c \\
\hline 12 & $2.16, \mathrm{~s}$ & $7.9^{\mathrm{b}}$ & 2.06 & 8.2 \\
\hline 13 & & 169.7 & & 170.1 \\
\hline 14 & & 129.3 & & 129.7 \\
\hline 15 & $6.46, \mathrm{~d}(11.2)$ & 138.0 & $6.46, \mathrm{~d}(11.3)$ & 138.6 \\
\hline 16 & $6.78, \mathrm{dd}(11.2,15.2)$ & 127.3 & 6.79, dd $(10.9,15.0)$ & 127.6 \\
\hline 17 & $6.02, \mathrm{dd}(8.0,15.2)$ & 145.4 & $6.03, \mathrm{dd}(8.3,15.0)$ & 146.0 \\
\hline 18 & $2.42, \mathrm{ddq}(9.1,8.0,6.9)$ & 42.0 & $2.43, \mathrm{~m}(8.3,7.5)$ & 42.4 \\
\hline 19 & $3.77, \mathrm{dd}(9.1,1.4)$ & 75.5 & $3.78, \mathrm{dd}(9.8,1.9)$ & 75.8 \\
\hline 20 & $1.86-1.89, \mathrm{~m}$ & 35.8 & $1.83, \mathrm{~m}(6.8,4.5,1.9)$ & 36.4 \\
\hline 21 & $3.51, \mathrm{dd}(8.3,4.3)$ & 77.9 & $3.50, \mathrm{dd}(8.3,4.5)$ & 78.4 \\
\hline 22 & $1.83-1.86, \mathrm{~m}$ & 36.7 & 1.82, br dq $(8.3,6.8,1.5)$ & 37.1 \\
\hline 23 & $3.95, \mathrm{dd}(10.6,1.1)$ & 74.7 & 3.94, br d $(10.5,1.5)$ & 75.2 \\
\hline 24 & $1.97-2.01, \mathrm{~m}$ & 35.0 & 1.97, br dq $(10.5,6.8,3.8)$ & 35.4 \\
\hline 25 & $4.22, \mathrm{dd}(6.7,3.4)$ & 81.3 & 4.20, br d $(6.8,3.8)$ & 81.8 \\
\hline $26 \mathbf{a}$ & $1.89-1.93, \mathrm{~m}$ & 24.6 & $1.93, \mathrm{~m}$ & 24.1 \\
\hline $26 b$ & $1.93-1.97, \mathrm{~m}$ & & $1.88, \mathrm{~m}$ & \\
\hline $27 \mathbf{a}$ & $1.78-1.83, \mathrm{~m}$ & 34.9 & $1.80, \mathrm{~m}$ & 35.4 \\
\hline $27 b$ & $2.01-2.05, \mathrm{~m}$ & & $2.01-2.05, \mathrm{~m}$ & \\
\hline 28 & & 106.2 & & 106.6 \\
\hline 29 & $2.08, \mathrm{~s}$ & 20.3 & $2.07, \mathrm{~m}(1.1)$ & 20.7 \\
\hline 30 & $0.99, \mathrm{~d}(6.9)$ & 16.7 & $0.99, \mathrm{~d}(6.8)$ & 17.1 \\
\hline 31 & $1.01, \mathrm{~d}(7.2)$ & 10.9 & $1.00, \mathrm{~d}(6.8)$ & 11.3 \\
\hline 32 & $0.89, \mathrm{~d}(7.0)$ & 10.1 & $0.88, \mathrm{~d}(6.8)$ & 10.5 \\
\hline 33 & $1.39, \mathrm{~s}$ & 24.0 & $1.39, \mathrm{~s}$ & 24.4 \\
\hline 34 & $0.73, \mathrm{~d}(6.9)$ & 12.6 & $0.71, \mathrm{~d}(6.8)$ & 13.0 \\
\hline
\end{tabular}

${ }^{a}$ Shifts not determined due to small amount of material. ${ }^{b}$ Shifts determined from HMBC. ${ }^{c}$ Carboxylate absent in salinisporamycin. 


\section{Experimental Procedures}

\section{$(3 S, 4 S)-3-M e t h y l o c t-1-e n-7-y n-4-o l ~(17)$}

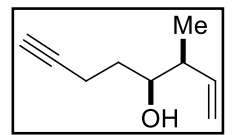

To a solution of pent-4-yn-1-ol $(1.68 \mathrm{~g}, 20 \mathrm{mmol})$ in $\mathrm{CH}_{2} \mathrm{Cl}_{2}(160 \mathrm{~mL})$ was added $\mathrm{NaHCO}_{3}(3.36 \mathrm{~g}, 40 \mathrm{mmol})$ and Dess-Martin periodinane $(17.68 \mathrm{~g}, 40 \mathrm{mmol})$ in one portion. The resulting mixture was allowed to stir for $2 \mathrm{~h}$ at RT. After removing the solvent, the residue was resolved in EtOAc $(150 \mathrm{~mL})$ and saturated aqueous $\mathrm{Na}_{2} \mathrm{~S}_{2} \mathrm{O}_{3}(100 \mathrm{~mL})$ and saturated aqueous $\mathrm{NaHCO}_{3}(50 \mathrm{~mL})$ were added. After stirring for $3 \mathrm{~h}$, the biphasic solution was separated. The aqueous phase was washed with ether $(100 \mathrm{~mL} \times 2)$ and the combined organic layers were washed with brine $(100$ $\mathrm{mL}$ ), dried over $\mathrm{MgSO}_{4}$, filtered, and concentrated in vacuum to provide the desired aldehyde as a colorless oil ( $1.54 \mathrm{~g}, 94 \%$, crude).

Potassium tert-butoxide (2.9 g, $26.0 \mathrm{mmol}, 2.0$ equiv) was heated at $80{ }^{\circ} \mathrm{C}$ under high vacuum overnight. Then, THF $(10 \mathrm{~mL})$ was added and the suspension was cooled to $-78^{\circ} \mathrm{C}$. Cis-2-butene $(3.3 \mathrm{~mL})$ was then added via cannula, followed by $n$-BuLi (1.6 $\mathrm{M}$ in hexane, $17.0 \mathrm{~mL}, 2.1$ equiv) dropwise to produce an orange suspension. After $10 \mathrm{~min}$ at $-45^{\circ} \mathrm{C}$, the reaction mixture was cooled to $-78{ }^{\circ} \mathrm{C}$, followed by the dropwise addition (15 min) of a solution of (+)-Ipc 2 BOMe (8.54 g, $27 \mathrm{mmol}, 2.05$ equiv) in THF (30 mL). After an additional $30 \mathrm{~min}$ at $-78^{\circ} \mathrm{C}$, the colorless slurry was treated with $\mathrm{BF}_{3} \cdot \mathrm{OEt}_{2}(3.7 \mathrm{~mL}, 29 \mathrm{mmol}$, 2.1 equiv; added dropwise over $10 \mathrm{~min}$ ), followed by a dropwise addition of a solution of the aboveprepared pent-4-ynal $(1.07 \mathrm{~g}, 13.0 \mathrm{mmol})$ in THF $(5 \mathrm{~mL}$ and $5 \mathrm{~mL}$ rinse). After an additional $3 \mathrm{~h} \mathrm{at} \mathrm{-}$ $78^{\circ} \mathrm{C}$, the pale yellow slurry was charged with $3 \mathrm{~N}$ aq. $\mathrm{NaOH}(20 \mathrm{~mL})$ and allowed to slowly warm to RT. During this time, $\mathrm{H}_{2} \mathrm{O}_{2}(30 \%$ aq., $6 \mathrm{~mL})$ was added in $1 \mathrm{~mL}$ portions to control gas evolution and the resulting mixture was heated at reflux for $1 \mathrm{~h}$. The biphasic solution was then cooled to ambient temperature and diluted with water $(30 \mathrm{~mL})$. The aqueous phase was then washed with ether $(30 \mathrm{~mL} \times 2)$ and the combined organic layers were washed with brine $(20 \mathrm{~mL})$, dried over $\mathrm{MgSO}_{4}$, filtered, and concentrated in vacuum. After FC (silica gel, $0 \rightarrow 10 \%$, EtOAc/hexanes), homoallylic alcohol 17 (1.56 g, $11.3 \mathrm{mmol}, 87 \%$ yield; >20:1 dr) was obtained.

Physical state: colorless oil.

TLC: $R_{f}=0.50(3: 1$, hexanes/EtOAc)

Optical Rotation: $[\alpha]_{D}^{20}=-22.3\left(c=2.0, \mathrm{CHCl}_{3}\right)$

${ }^{1} \mathbf{H}$ NMR $\left(400 \mathrm{MHz}, \mathrm{CDCl}_{3}\right) \delta$ 5.72-5.82 (m, 1H), 5.06-5.13 (m, 2H), 3.56-3.68 (m, 1H), 2.24-2.39 (m, $3 \mathrm{H}), 1.91(\mathrm{t}, J=2.6 \mathrm{~Hz}, 1 \mathrm{H}), 1.70-1.80(\mathrm{~m}, 1 \mathrm{H}), 1.48-1.62(\mathrm{~m}, 2 \mathrm{H}), 1.04(\mathrm{~d}, J=6.9 \mathrm{~Hz}, 3 \mathrm{H})$

${ }^{13}$ C NMR $\left(100 \mathrm{MHz}, \mathrm{CDCl}_{3}\right) \delta 140.6,115.9,84.5,73.7,68.8,43.8,32.7,15.5,14.6$

MS (ES) calculated for $\mathrm{C}_{9} \mathrm{H}_{15} \mathrm{O}[\mathrm{M}+\mathrm{H}]^{+} 139.1$, found 139.1 


\section{Triethyl(((3S,4S)-3-methyloct-1-en-7-yn-4-yl)oxy)silane (S1)}

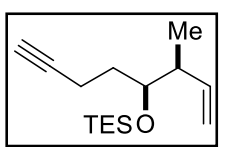

To a solution of homoallylic alcohol $17(1.38 \mathrm{~g}, 10 \mathrm{mmol})$ in $\mathrm{CH}_{2} \mathrm{Cl}_{2}(25 \mathrm{~mL})$ was added triethylsilyl chloride $(3.01 \mathrm{~g}, 20 \mathrm{mmol})$, imidazole $(2.04 \mathrm{~g}, 30 \mathrm{mmol})$ and DMAP $(610 \mathrm{mg}$, $5 \mathrm{mmol})$ at $0{ }^{\circ} \mathrm{C}$. After stirring for 2 hours at RT, saturated aqueous $\mathrm{NaHCO}_{3}(10 \mathrm{~mL})$ was added. The biphasic solution was separated and the aqueous phase was washed with ethyl acetate $(10 \mathrm{~mL}$ $\times 3)$. The combined organic layers were washed with brine $(20 \mathrm{~mL})$, dried over $\mathrm{MgSO}_{4}$, filtered, and concentrated in vacuo. After FC (silica gel, 0 $\rightarrow 2 \%$, EtOAc/hexanes), the desired silyl ether S1 (2.4 g, 9.5 mmol, 95\% yield) was obtained.

Physical state: colorless oil

TLC: $R_{f}=0.4(20: 1$, hexanes/EtOAc)

Optical rotation: $[\alpha]_{D}^{20}=-19.5\left(c=1.0, \mathrm{CHCl}_{3}\right)$

${ }^{1}$ H NMR (400 MHz, $\left.\mathrm{CDCl}_{3}\right) \delta$ 5.76-5.83 (m, 1H), 4.90-5.06 (m, 2H), 3.62-3.67 (m, 1H), 2.15-2.35 (m, $3 \mathrm{H}), 1.91(\mathrm{t}, J=2.6 \mathrm{~Hz}, 1 \mathrm{H}), 1.50-1.70(\mathrm{~m}, 2 \mathrm{H}), 0.95-0.97(\mathrm{~m}, 9 \mathrm{H}), 0.61(\mathrm{q}, J=8.0 \mathrm{~Hz}, 6 \mathrm{H})$

${ }^{13}$ C NMR $\left(100 \mathrm{MHz}, \mathrm{CDCl}_{3}\right) \delta 140.7,114.6,84.8,74.8,68.4,43.2,32.5,15.4,14.7,7.2,5.3$

MS (ES) calculated for $\mathrm{C}_{15} \mathrm{H}_{29} \mathrm{OSi}[\mathrm{M}+\mathrm{H}]^{+} 253.2$, found 253.2

(2R,3S)-2-Methyl-3-((triethylsilyl)oxy)hept-6-ynal (13)

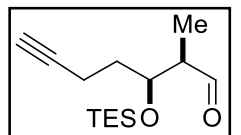

To a solution of the above-prepared alkene $\mathbf{S 1}(2.016 \mathrm{~g}, 8.0 \mathrm{mmol})$ in acetone $/ \mathrm{H}_{2} \mathrm{O}(40 \mathrm{~mL}$, 10:1) was added $\mathrm{OsO}_{4}(0.4 \mathrm{mmol}, 4.0 \mathrm{~mL}, 0.1 \mathrm{M}$ in $t-\mathrm{BuOH})$ and $\mathrm{NMO}(1.84 \mathrm{~g}, 16.0$ mmol) and the mixture was stirred for 2 hours at RT. After removal of he solvents in vacuo, the crude residue was dissolved in $\mathrm{CH}_{2} \mathrm{Cl}_{2}(40 \mathrm{~mL})$, and $\mathrm{Pb}(\mathrm{OAc})_{4}(5.316 \mathrm{~g}, 12 \mathrm{mmol}, 1.5 \mathrm{eq})$ and pyridine (3.0 eq) were added at $0{ }^{\circ} \mathrm{C}$. After stirring for overnight at $\mathrm{RT}$, aq. $\mathrm{CuSO}_{4}(20 \mathrm{~mL})$ was added. The biphasic solution was then separated. The aqueous phase was washed with ethyl acetate $(10 \mathrm{~mL} \times 3)$ and the combined organic layers were washed with brine $(20 \mathrm{~mL})$, dried over $\mathrm{MgSO}_{4}$, filtered, and concentrated in vacuo. After FC (silica gel, $0 \rightarrow 10 \%$, EtOAc/hexanes), the desired product 13 (1.73 g, $6.8 \mathrm{mmol}, 85 \%$ yield) was obtained.

Physical state: pale yellow oil

TLC: $R_{f}=0.35(2: 1$, hexanes/EtOAc)

Optical rotation: $[\alpha]_{D}^{20}=-24.8\left(c=1.0, \mathrm{CHCl}_{3}\right)$

${ }^{1}$ H NMR $\left(400 \mathrm{MHz}, \mathrm{CDCl}_{3}\right) \delta 9.81(\mathrm{~s}, 1 \mathrm{H}), 4.26(\mathrm{ddd}, J=7.5,5.5,3.8 \mathrm{~Hz}, 1 \mathrm{H}), 2.50(\mathrm{qd}, J=7.0,3.5 \mathrm{~Hz}$, 1H), $2.24(\mathrm{td}, J=7.1,2.7 \mathrm{~Hz}, 2 \mathrm{H}), 1.97(\mathrm{t}, J=2.7 \mathrm{~Hz}, 1 \mathrm{H}), 1.58-1.76(\mathrm{~m}, 2 \mathrm{H}), 1.06(\mathrm{~d}, J=7.0 \mathrm{~Hz}, 3 \mathrm{H})$, $0.94(\mathrm{t}, J=7.9 \mathrm{~Hz}, 9 \mathrm{H}), 0.61(\mathrm{q}, J=8.2 \mathrm{~Hz}, 6 \mathrm{H})$

${ }^{13} \mathbf{C}$ NMR $\left(100 \mathrm{MHz}, \mathrm{CDCl}_{3}\right) \delta 204.9,83.8,70.9,69.1,51.4,32.9,14.9,8.0,6.9,5.0$

MS (ES) calculated for $\mathrm{C}_{14} \mathrm{H}_{27} \mathrm{O}_{2} \mathrm{Si}[\mathrm{M}+\mathrm{H}]^{+} 255.2$, found 255.2 
(4R,5S,6S,7S)-5-Hydroxy-4, 6-dimethyl-7((triethylsilyl)oxy)undec-10-yn-3-one (18-syn)

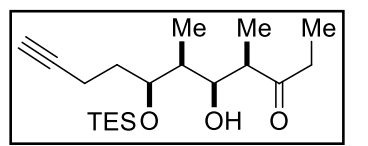

$\mathrm{TiCl}_{4}\left(1.0 \mathrm{M}\right.$ in $\left.\mathrm{CH}_{2} \mathrm{Cl}_{2} ; 1.2 \mathrm{~mL}\right)$ and $\mathrm{Bu}_{3} \mathrm{~N}(185 \mathrm{mg}, 1.4 \mathrm{mmol})$ were successively added to a stirred solution of 3-pentanone (134 mg, $1.0 \mathrm{mmol})$ in $\mathrm{CH}_{2} \mathrm{Cl}_{2}(2.0 \mathrm{~mL})$ at $-78{ }^{\circ} \mathrm{C}$ under an $\mathrm{Ar}$ atmosphere. After $30 \mathrm{~min}$, aldehyde 13 (305 $\mathrm{mg}, 1.2 \mathrm{mmol}$ ) was added to the mixture, which was stirred at $-78{ }^{\circ} \mathrm{C}$ for $2 \mathrm{~h}$. The reaction mixture was quenched with water $(2 \mathrm{~mL})$ and was extracted ether $(5 \mathrm{~mL} \times 3)$. The organic phase was washed with water, brine, dried with $\mathrm{MgSO}_{4}$ and concentrated in vacuo. The obtained crude oil was purified by FC (silica gel, $0 \rightarrow 10 \%$, EtOAc/hexanes) to give the aldol product 18-syn (192 $\mathrm{mg}, 53 \%)$.

Note: This experiment was repeated two times with yields varying between 43-53\%. When two equivalents of titanium enolate were used, the yields varied between $37-46 \%$ yield. In all cases, the ratio of 18-syn versus other diastereomers was $>10: 1$ (measured from crude NMR). Varying amounts (TLC) of compound 19 were observed during all of the above experiments. In one case, we obtained an analytically pure sample for characterization.

\section{Characterization data for 18-syn:}

Physical state: pale-yellow oil

TLC: $R_{f}=0.30(5: 1$, hexanes/EtOAc)

Optical rotation: $[\alpha]_{D}^{20}=-24.2\left(c=1.03\right.$ in $\left.\mathrm{CDCl}_{3}\right)$

${ }^{1}$ H NMR $\left(400 \mathrm{MHz}, \mathrm{CDCl}_{3}\right) \delta$ 3.87-3.97 (m, 2H), 3.08 (br s, 1H), 2.82 (dq, J= 7.0, 7.0 Hz, 1H), 2.36$2.62(\mathrm{~m}, 2 \mathrm{H}), 2.06-2.18(\mathrm{~m}, 2 \mathrm{H}), 1.95($ br s, $1 \mathrm{H}), 1.63-1.76(\mathrm{~m}, 2 \mathrm{H}), 1.50-1.58(\mathrm{~m}, 1 \mathrm{H}), 1.17(\mathrm{~d}, J=7.0$ $\mathrm{Hz}, 3 \mathrm{H}), 1.04(\mathrm{t}, J=7.6 \mathrm{~Hz}, 3 \mathrm{H}), 0.95$ (t, $J=7.9 \mathrm{~Hz}, 9 \mathrm{H}), 0.90(\mathrm{~d}, J=6.8 \mathrm{~Hz}, 3 \mathrm{H}), 0.62(\mathrm{q}, J=7.9 \mathrm{~Hz}$, $6 \mathrm{H})$

${ }^{13} \mathbf{C}$ NMR $\left(100 \mathrm{MHz}, \mathrm{CDCl}_{3}\right) \delta 215.8,83.5,75.3,74.5,69.1,49.2,38.2,35.4,32.7,15.1,12.7,7.9,7.6$, $7.1,5.5$

MS (ES) calculated for $\mathrm{C}_{19} \mathrm{H}_{36} \mathrm{O}_{3} \mathrm{SiNa}[\mathrm{M}+\mathrm{Na}]^{+}$363.2, found 363.2

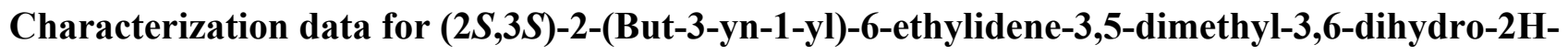
pyran (19):

Physical state: pale yellow oil

TLC: $R_{f}=0.80(5: 1$, hexanes/EtOAc)

Optical rotation: $[\alpha]_{D}^{20}=-11.2\left(c=0.25\right.$ in $\left.\mathrm{CH}_{2} \mathrm{Cl}_{2}\right)$

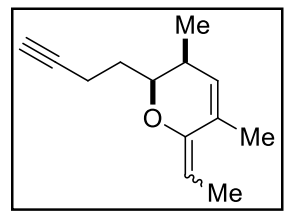

${ }^{1}$ H NMR $\left(400 \mathrm{MHz}, \mathrm{CDCl}_{3}\right) \delta 5.58(\mathrm{~d}, J=5.7 \mathrm{~Hz}, 1 \mathrm{H}), 4.69(\mathrm{q}, J=6.9, \mathrm{~Hz}, 1 \mathrm{H}), 3.91(\mathrm{dt}, J=10.1,3.4$ $\mathrm{Hz}, 1 \mathrm{H}), 2.26-2.50(\mathrm{~m}, 2 \mathrm{H}), 2.17-2.25(\mathrm{~m}, 1 \mathrm{H}), 1.94(\mathrm{t}, J=2.8 \mathrm{~Hz}, 1 \mathrm{H}), 1.80-1.87(\mathrm{~m}, 1 \mathrm{H}), 1.74(\mathrm{~s}, 3 \mathrm{H})$, $1.68(\mathrm{~d}, J=7.2 \mathrm{~Hz}, 3 \mathrm{H}), 1.52-1.65(\mathrm{~m}, 1 \mathrm{H}), 0.84(\mathrm{~d}, J=6.8 \mathrm{~Hz}, 3 \mathrm{H})$

MS (ES) calculated for $\mathrm{C}_{13} \mathrm{H}_{18} \mathrm{ONa}[\mathrm{M}+\mathrm{Na}]^{+} 213.1$, found 213.2 
Aldol coupling of aldehyde 13 and oxazolidinone 20: $\mathrm{TiCl}_{4}\left(1 \mathrm{M}\right.$ in $\left.\mathrm{CH}_{2} \mathrm{Cl}_{2}, 0.671 \mathrm{~mL}\right)$ was added dropwise to a solution of N-propionyl-4-benzyl-oxazolidininone 20 (142 mg, $0.61 \mathrm{mmol})$ in $\mathrm{CH}_{2} \mathrm{Cl}_{2}(3$ $\mathrm{mL})$ at $0{ }^{\circ} \mathrm{C}$. After stirring for $5 \mathrm{~min},(-)$-sparteine $(350 \mu \mathrm{L}, 1.53 \mathrm{mmol})$ was added and stirring was continued for $20 \mathrm{~min}$. A solution of aldehyde $13(170 \mathrm{mg}, 0.67 \mathrm{mmol})$ in $\mathrm{CH}_{2} \mathrm{Cl}_{2}(3 \mathrm{~mL})$ was added and stirred for $1 \mathrm{~h}$ at $0{ }^{\circ} \mathrm{C}$. The mixture was quenched with saturated aqueous $\mathrm{NH}_{4} \mathrm{Cl}(10 \mathrm{~mL})$ and $\mathrm{CH}_{2} \mathrm{Cl}_{2}(10$ $\mathrm{mL})$. The aqueous phase was extracted with EtOAc $(10 \mathrm{~mL} \times 3)$. The combined organic phase was dried over $\mathrm{MgSO}_{4}$. After concentration in vacuo, the crude residue was purified by FC (silica gel, $0 \rightarrow 10 \%$, EtOAc/hexanes) to give compound 21-anti (100 mg, 34\%) and 21-syn (120 mg, 39\%).

Characterization data for $(S)-4-B e n z y l-3-((2 S, 3 R, 4 S, 5 S)-3-h y d r o x y-2,4-d i m e t h y l-5-((t r i e t h y l s i l y l)-$ oxy)non-8-ynoyl)oxazolidin-2-one (21-anti):

Physical state: pale yellow solid

TLC: $R_{f}=0.50(3: 1$, hexanes/EtOAc)

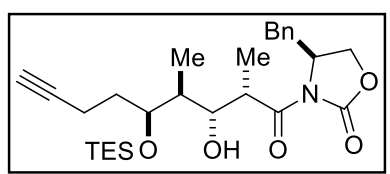

Optical rotation: $[\alpha]_{D}^{20}=+35.5\left(c=0.8\right.$ in $\left.\mathrm{CDCl}_{3}\right)$

${ }^{1}$ H NMR $\left(400 \mathrm{MHz}, \mathrm{CDCl}_{3}\right) \delta$ 7.13-7.37 (m, 5H), 4.67 (dtd, $\left.J=10.1,7.5,2.8 \mathrm{~Hz}, 1 \mathrm{H}\right), 4.34(\mathrm{~s}, 1 \mathrm{H}), 4.13-$ $4.25(\mathrm{~m}, 2 \mathrm{H}), 3.98-4.05(\mathrm{~m}, 2 \mathrm{H}), 3.86(\mathrm{qd}, J=6.9,2.4 \mathrm{~Hz}, 1 \mathrm{H}), 3.35(\mathrm{dd}, J=13.3,2.8 \mathrm{~Hz}, 1 \mathrm{H}), 2.75(\mathrm{dd}$, $J=13.3,10.1 \mathrm{~Hz} 1 \mathrm{H}), 2.25-2.38(\mathrm{~m}, 1 \mathrm{H}), 2.08-2.23(\mathrm{~m}, 1 \mathrm{H}), 1.95$ (br s, 1H), 1.80-1.87 (m, 1H), $1.65-$ $1.76(\mathrm{~m}, 2 \mathrm{H}), 1.21(\mathrm{~d}, J=6.8 \mathrm{~Hz}, 3 \mathrm{H}), 0.96(\mathrm{t}, J=8.0 \mathrm{~Hz}, 9 \mathrm{H}), 0.85(\mathrm{~d}, J=6.0 \mathrm{~Hz}, 3 \mathrm{H}), 0.64(\mathrm{q}, J=8.0$ $\mathrm{Hz}, 6 \mathrm{H})$

${ }^{13} \mathbf{C}$ NMR $\left(100 \mathrm{MHz}, \mathrm{CDCl}_{3}\right) \delta 175.2,153.5,135.7,129.6,129.1,127.5,84.0,75.6,73.3,69.1,66.4,56.1$, $41.0,39.9,37.9,30.8,15.6,12.5,8.6,7.0,5.1$

MS (ES) calculated for $\mathrm{C}_{27} \mathrm{H}_{42} \mathrm{NO}_{5} \mathrm{Si}[\mathrm{M}+\mathrm{H}]^{+} 488.3$, found 488.3

Characterization data for $(S)-4-B e n z y l-3-((2 R, 3 S, 4 S, 5 S)-3-h y d r o x y-2,4-d i m e t h y l-5-((t r i e t h y l s i l y l)-$ oxy)non-8-ynoyl)-oxazolidin-2-one (21-syn):

Physical state: pale yellow solid

TLC: $R_{f}=0.35$ (3:1, hexanes:EtOAc)

Optical rotation: $[\alpha]_{D}^{20}=-27.8\left(c=1.0\right.$ in $\left.\mathrm{CH}_{2} \mathrm{Cl}_{2}\right)$

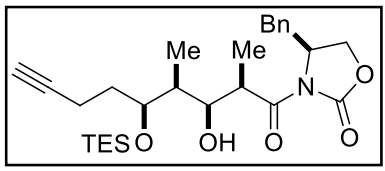

${ }^{1}$ H NMR $\left(400 \mathrm{MHz}, \mathrm{CDCl}_{3}\right) \delta$ 7.12-7.37 (m, 5H), 4.65 (ddt, $\left.J=10.3,6.8,3.2 \mathrm{~Hz}, 1 \mathrm{H}\right), 3.93-4.23(\mathrm{~m}, 5 \mathrm{H})$, $3.32(\mathrm{dd}, J=13.3,3.4 \mathrm{~Hz}, 1 \mathrm{H}), 3.19(\mathrm{~s}, 1 \mathrm{H}), 2.66(\mathrm{dd}, J=13.2,10.0 \mathrm{~Hz}, 1 \mathrm{H}), 2.10-2.23(\mathrm{~m}, 2 \mathrm{H}), 1.94(\mathrm{t}$, $J=2.6 \mathrm{~Hz}, 1 \mathrm{H}), 1.74(\mathrm{dt}, J=7.1,7.0 \mathrm{~Hz}, 2 \mathrm{H}), 1.64-1.70(\mathrm{~m}, 1 \mathrm{H}), 1.28(\mathrm{~d}, J=6.2 \mathrm{~Hz}, 3 \mathrm{H}), 0.96(\mathrm{t}, J=8.0$ $\mathrm{Hz}, 9 \mathrm{H}), 0.94(\mathrm{~d}, J=8.0 \mathrm{~Hz}, 3 \mathrm{H}), 0.63$ (q, $J=7.9 \mathrm{~Hz}, 6 \mathrm{H})$

${ }^{13}$ C NMR $\left(100 \mathrm{MHz}, \mathrm{CDCl}_{3}\right) \delta 177.3,153.0,135.4,129.5,129.2,127.6,83.4,74.9,74.6,69.2,66.1,55.5$, $41.0,38.4,38.2,32.7,15.0,13.5,7.6,7.1,5.4$

MS (ES) calculated for $\mathrm{C}_{27} \mathrm{H}_{42} \mathrm{NO}_{5} \mathrm{Si}[\mathrm{M}+\mathrm{H}]^{+} 488.3$, found 488.3 
Acetonides 23-anti or 23-syn: PPTS (10 mol\%) was added to a solution of aldol adduct 21-anti or 21-syn in $\operatorname{EtOH}(0.03 \mathrm{M})$ and stirred at room temperature until TLC analysis indicated the reaction was complete. After concentration in vacuo, the crude residue was dissolved in acetone/2,2-dimethoxypropane (4:1, 0.03 M) and PPTS (6 mol\%) was added. This mixture was stirred at room temperature for $30 \mathrm{~min}$; quenched by the addition of $\mathrm{Et}_{3} \mathrm{~N}$ (1 equiv.), then concentrated in vacuo. The crude was purified by FC (silica gel, $0 \rightarrow 20 \%$, EtOAc/hexanes) to give compound 23-anti or 23-syn.

Characterization data for $(S)-4-b e n z y l-3-((S)-2-((4 R, 5 R, 6 S)-6-(b u t-3-y n-1-y l)-2,2,5-t r i m e t h y l-1,3-$ dioxan-4-yl)propanoyl)oxazolidin-2-one (23-anti):

Physical state: yellow solid

TLC: $R_{f}=0.55(3: 1$, hexanes/EtOAc)

Optical rotation: $[\alpha]_{D}^{20}=46.2\left(c=1.43\right.$ in $\left.\mathrm{CH}_{2} \mathrm{Cl}_{2}\right)$

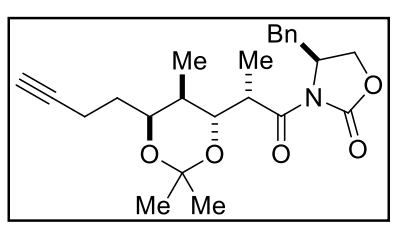

${ }^{1}$ H NMR (400 MHz, $\left.\mathrm{CDCl}_{3}\right) \delta: 7.34-7.18(\mathrm{~m}, 5 \mathrm{H}), 4.62(\mathrm{dq}, J=6.4,3.2,1 \mathrm{H}), 4.18-4.11(\mathrm{~m}, 2 \mathrm{H}), 4.04-$ $3.98(\mathrm{~m}, 1 \mathrm{H}), 3.92(\mathrm{dt}, J=10.4,3.2,1 \mathrm{H}), 3.60(\mathrm{dd}, J=7.2,4.8,1 \mathrm{H}), 3.33(\mathrm{dd}, J=13.2,3.2,1 \mathrm{H}), 2.76(\mathrm{dd}$, $J=13.2,9.6,1 \mathrm{H}), 2.34-2.17$ (m, 2H), 1.93 (br s, 1H), 1.91-1.84 (m, 1H), 1.68-1.61 (m, 1H), 1.53-1.43 (m, $1 \mathrm{H}), 1.32(\mathrm{~s}, 3 \mathrm{H}), 1.28(\mathrm{~s}, 3 \mathrm{H}), 1.26(\mathrm{~d}, J=6.8,3 \mathrm{H}), 0.89(\mathrm{~d}, J=6.8,3 \mathrm{H})$

${ }^{13}$ C NMR $\left(75 \mathrm{MHz}, \mathrm{CDCl}_{3}\right) \delta 175.0,153.4,135.5,129.7,129.1,127.5,100.7,84.2,75.4,68.7,68.0,66.3$, 56.1, 41.2, 38.0, 37.0, 30.0, 25.2, 23.9, 15.3, 12.3, 11.8; IR (film, $\mathrm{cm}^{-1}$ ): 3290, 2936, 1781, 1698, 1382, 1210

MS (ES) calculated for $\mathrm{C}_{24} \mathrm{H}_{32} \mathrm{NO}_{5}[\mathrm{M}+\mathrm{H}]^{+} 414.2$; found 414.2

Note: The spectra of this acetonide are identical to the spectra of the acetonide of 22-anti synthesized from 30 (Scheme 5B). [Liu, J.; De Brabander, J. K. J. Am. Chem. Soc. 2009, 131, 12562-12563]

Characterization data for $(S)-4-b e n z y l-3-((R)-2-((4 S, 5 R, 6 S)-6-(b u t-3-y n-1-y l)-2,2,5-t r i m e t h y l-1,3-$ dioxan-4-yl)propanoyl)oxazolidin-2-one (23-syn):

Physical state: yellow solid

TLC: $R_{f}=0.60(3: 1$, hexanes/EtOAc)

Optical rotation: $[\alpha]_{D}^{20}=-20.2\left(c=0.85\right.$ in $\left.\mathrm{CH}_{2} \mathrm{Cl}_{2}\right)$

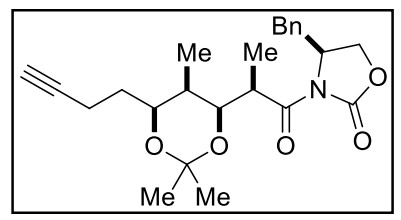

${ }^{1} \mathbf{H}$ NMR $\left(400 \mathrm{MHz}, \mathrm{CDCl}_{3}\right) \delta$ 7.18-7.36 (m, 5H), 4.62-4.70 (m, 1H), 4.13-4.25 (m, 3H), 3.94-4.10 (m, 2H), 3.27 (dd, $J=13.3,3.6 \mathrm{~Hz}, 1 \mathrm{H}), 2.71$ (dd, $J=13.4,9.6 \mathrm{~Hz}, 1 \mathrm{H}), 2.14-2.32$ (m, 2H), 1.90 (br s, 1H), 1.69-1.80 (m, 1H), 1.40-1.53 (m, 2H), $1.45(\mathrm{~s}, 3 \mathrm{H}), 1.38(\mathrm{~s}, 3 \mathrm{H}), 1.25(\mathrm{~d}, J=6.7 \mathrm{~Hz}, 3 \mathrm{H}), 0.87(\mathrm{~d}, J=$ $13.2 \mathrm{~Hz}, 3 \mathrm{H})$

${ }^{13}$ C NMR $\left(100 \mathrm{MHz}, \mathrm{CDCl}_{3}\right) \delta 175.5,152.9,135.2,129.6,129.2,127.6,99.6,84.2,75.2,71.3,68.6,66.1$, $55.4,39.9,38.4,33.4,31.8, \mathbf{3 0 . 1}, \mathbf{1 9 . 8}, 15.9,15.0,6.0$

MS (ES) calculated for $\mathrm{C}_{24} \mathrm{H}_{32} \mathrm{NO}_{5}[\mathrm{M}+\mathrm{H}]^{+} 414.2$, found 414.3 
Preparation of ethyl ketone 18-syn from 21-syn: To a stirred solution of syn aldol product 21-syn (25 $\mathrm{mg}, 0.05 \mathrm{mmol})$ in $\mathrm{CH}_{2} \mathrm{Cl}_{2}(3 \mathrm{~mL})$ at $-78{ }^{\circ} \mathrm{C}$ was added EtMgBr $(2 \mathrm{M}$ in THF, 3.5 equiv, $0.175 \mathrm{mmol}$, $87.5 \mu \mathrm{L})$. After stirring at that temperature for $6 \mathrm{~h}$, the reaction was quenched by the addition of water, followed by extraction with $\mathrm{CH}_{2} \mathrm{Cl}_{2}$, washing the organic phase with aqueous saturated $\mathrm{NH}_{4} \mathrm{Cl}$, and drying over $\mathrm{MgSO}_{4}$. After removal of the solvent, the residue was purified by FC (silica gel, $2 \% \rightarrow 20 \%$, EtOAc/hexanes) to yield ethyl ketone 18-syn (4.7 mg, 33\%) as a pale yellow oil. The spectra of this ethyl ketone are identical to the spectra of ethyl ketone 18-syn synthesized from 13 (Scheme 3).

$\left[{ }^{1} \mathrm{H}-\mathrm{NMR}\right.$ analysis of the crude mixture indicated that the remainder of the mass balance was primarily the diethyl carbinol from over-addition. We have not attempted to purify or characterize this byproduct].

(2R,4R,5S)-1-(Benzyloxy)-5-hydroxy-2,4-dimethylnon-8-yn-3-one (26)

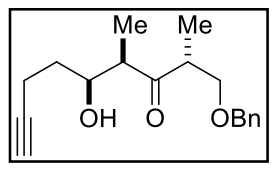

To a solution of $(+)$-diisopinocampheylboron triflate $(5.1 \mathrm{mmol})$ and $i-\operatorname{Pr}_{2} \mathrm{NEt}(1.76 \mathrm{~mL}$, $10.2 \mathrm{mmol})$ in $\mathrm{CH}_{2} \mathrm{Cl}_{2}(5 \mathrm{~mL})$ at $-78{ }^{\circ} \mathrm{C}$ was added dropwise a solution of ketone 25 (700 mg, $3.4 \mathrm{mmol}$ ) [prepared according to: Paterson, I.; Razzak, M.; Anderson, E. A. Org. Lett. 2008, 10, 3295-3298] in $\mathrm{CH}_{2} \mathrm{Cl}_{2}\left(2 \mathrm{~mL}+1 \mathrm{~mL}\right.$ washing). The reaction was stirred at $-78{ }^{\circ} \mathrm{C}$ for $15 \mathrm{~min}$, then warmed to $0{ }^{\circ} \mathrm{C}$ and stirred for $2 \mathrm{~h}$. The enolate solution was recooled to $-78^{\circ} \mathrm{C}$ and pent-4ynal 24 (836 mg, $10.2 \mathrm{mmol})$ was added. The reaction was stirred at $-78{ }^{\circ} \mathrm{C}$ for $3 \mathrm{~h}$ and placed in the freezer $\left(-23^{\circ} \mathrm{C}\right)$ for $16 \mathrm{~h}$. The reaction was then warmed to $0{ }^{\circ} \mathrm{C}$ and quenched with excess $\mathrm{MeOH}(7 \mathrm{~mL})$ and $\mathrm{pH} 7$ phosphate buffer $(7 \mathrm{~mL})$. Hydrogen peroxide solution $(30 \%$ aqueous, $3.5 \mathrm{~mL}, 31 \mathrm{mmol})$ was then added dropwise and the reaction stirred for $1 \mathrm{~h}$, with warming to RT. The mixture was extracted with $\mathrm{CH}_{2} \mathrm{Cl}_{2}(10 \mathrm{~mL} \times 4)$ and the combined organic fractions were washed with saturated aqueous $\mathrm{NaHCO}_{3}$, dried $\left(\mathrm{MgSO}_{4}\right)$, filtered and concentrated in vacuo to remove residual solvent. The crude was purified by FC (silica gel, $0 \rightarrow 10 \%$ EtOAc/hexanes) to give the aldol adduct 26 (813 $\mathrm{mg}, 83 \%$ ).

Physical state: pale yellow oil

TLC: $R_{f}=0.30(3: 7$, EtOAc/hexanes)

Optical rotation: $[\alpha]_{D}^{20}=-16.2(c=1.0$ in EtOAc $)$

${ }^{1}$ H NMR $\left(400 \mathrm{MHz}, \mathrm{CDCl}_{3}\right) \delta$ 7.20-7.38 (m, 5H), $4.45(\mathrm{~s}, 2 \mathrm{H}), 4.14(\mathrm{br} \mathrm{d}, J=9.7 \mathrm{~Hz}, 1 \mathrm{H}), 3.64(\mathrm{dd}, J=$ 9.4, 8.4 Hz, 1H), 3.40-3.50 (m, 1H), 3.18 (dqd, $J=9.6,7.0,4.6 \mathrm{~Hz}, 1 \mathrm{H}), 2.90$ (br s, 1H), 2.68-2.78 (m, 1H), 2.18-2.37 (m, 2H), 1.91 (br s, 1H), 1.56-1.72 (m, 1H), 1.44-1.55 (dtd, $J=13.9,7.8,3.5 \mathrm{~Hz}, 1 \mathrm{H}), 1.06$ $(\mathrm{d}, J=7.0 \mathrm{~Hz}, 3 \mathrm{H}), 1.00(\mathrm{~d}, J=6.9 \mathrm{~Hz}, 3 \mathrm{H})$

${ }^{13}$ C NMR $\left(100 \mathrm{MHz}, \mathrm{CDCl}_{3}\right) \delta 217.7,137.4,128.5,127.9,127.8,84.0,73.5,73.3,69.4,68.5,51.1,44.8$, $32.3,15.2,13.6,9.0$

MS (ES) calculated for $\mathrm{C}_{18} \mathrm{H}_{25} \mathrm{O}_{3}[\mathrm{M}+\mathrm{H}]^{+}, 289.2$, found: 289.2 


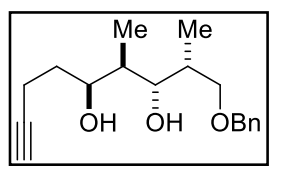

To $5 \mathrm{~mL}$ of acetic acid at $0{ }^{\circ} \mathrm{C}$ was added portionwise $\mathrm{NaBH}_{4}(293 \mathrm{mg}, 7.7 \mathrm{mmol})$. After completion of gas evolution (about $10 \mathrm{~min}$ ), the reaction was allowed to warm to RT and stirred for $1 \mathrm{~h}$. To this solution was added a solution of $\beta$-hydroxy ketone $\mathbf{2 6}$ (373 $\mathrm{mg}$ in $2.5 \mathrm{~mL}$ acetic acid, $0.77 \mathrm{mmol}$ ). After $70 \mathrm{~min}$, the reaction was concentrated in vacuo. The residue was poured into saturated aqueous $\mathrm{NaHCO}_{3}\left(25 \mathrm{~mL}\right.$, caution!). The aqueous layer was extracted with $\mathrm{CH}_{2} \mathrm{Cl}_{2}$ $(30 \mathrm{~mL} \times 3)$ and the combined organic phase was washed with brine, dried over $\mathrm{Na}_{2} \mathrm{SO}_{4}$ and concentrated in vacuo. The crude residue (300 mg, 80\%) was used as such in the next step (see general procedure for cycloisomerization of alkynediols).

\section{General procedure for the cycloisomerization of alkynediols}

To a solution of alkynediol (22-syn, 22-anti, 27, or 31-syn) in freshly distilled THF (0.1 M) was added Zeise's dimer $\left(\left[\mathrm{Pt}\left(\mathrm{CH}_{2} \mathrm{CH}_{2}\right) \mathrm{Cl}_{2}\right]_{2}, 5 \mathrm{~mol} \%\right)$ and the solution was stirred at room temperature for 5 min to $1.5 \mathrm{~h}$. When the reaction was complete, it was quenched with $300 \mu \mathrm{L}_{\text {of }} \mathrm{NEt}_{3}$ per $\mathrm{mL}$ of THF solvent. The mixture was concentrated in vacuo and purified by FC (silica gel, $0 \rightarrow 35 \%$, EtOAc/hexanes) to give the compounds 32-35 with yields as reported in Table 1.

Characterization data for $(1 S, 3 S, 4 R, 5 S)-3-((R)-1-(B e n z y l o x y) p r o p a n-2-y l)-1,4-d i m e t h y l-2,8-d i o x a b i-$ cyclo[3.2.1]octane (32):

Physical state: yellow oil (95\% yield from crude 27)

TLC: $R_{f}=0.25(1: 10$, EtOAc/hexanes $)$

Optical rotation: $[\alpha]_{D}^{20}=-9.2\left(c=0.8\right.$ in $\left.\mathrm{CH}_{2} \mathrm{Cl}_{2}\right)$

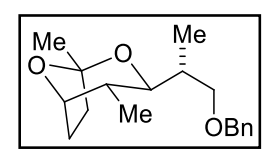

${ }^{1} \mathbf{H}$ NMR $\left(500 \mathrm{MHz}, \mathrm{CDCl}_{3}\right) \delta$ 7.24-7.34 (m, 5H), $4.49(\mathrm{~d}, J=12.0 \mathrm{~Hz}, 1 \mathrm{H}), 4.47(\mathrm{~d}, J=12.0 \mathrm{~Hz}, 1 \mathrm{H})$, 4.15 (m, 1H), 3.67 (dd, $J=11.2,4.8 \mathrm{~Hz}, 1 \mathrm{H}), 3.33$ (br d, $J=11.5 \mathrm{~Hz}, 1 \mathrm{H}), 3.28(\mathrm{dd}, J=11.2,7.2 \mathrm{~Hz}, 1 \mathrm{H})$, 2.13-1.74 (m, 6H), $1.46(\mathrm{~s}, 3 \mathrm{H}), 1.02(\mathrm{~d}, J=6.9 \mathrm{~Hz}, 3 \mathrm{H}), 0.73(\mathrm{~d}, J=6.9 \mathrm{~Hz}, 3 \mathrm{H})$

${ }^{13} \mathbf{C}$ NMR $\left(125 \mathrm{MHz}, \mathrm{CDCl}_{3}\right) \delta 138.9,128.6,127.4,105.0,80.1,77.9,73.7,71.9,35.2,34.7,33.9,24.0$, 24.0, $16.6,13.5$

MS (ES) calculated for $\mathrm{C}_{18} \mathrm{H}_{26} \mathrm{O}_{3} \mathrm{Na}[\mathrm{M}+\mathrm{Na}]^{+} 313.2$, found 313.2

Characterization data for $(2 R, 3 S, 4 R, 5 S)-1-((S)-4-B e n z y l-2-0 x 00 x a z o l i d i n-3-y l)-3,5-d i h y d r o x y-2,4-$ dimethylnonane-1,8-dione (34):

Physical state: yellow solid ( $81 \%$ yield)

TLC: $R_{f}=0.18(1: 1$, hexanes/EtOAc)

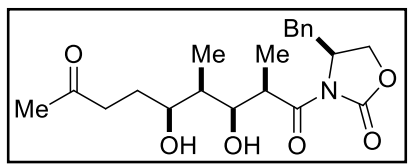

Optical rotation: $[\alpha]_{D}^{20}=+12.5\left(c=0.20\right.$ in $\left.\mathrm{CH}_{2} \mathrm{Cl}_{2}\right)$ 
${ }^{1}$ H NMR $\left(400 \mathrm{MHz}, \mathrm{CDCl}_{3}\right) \delta$ 7.13-7.35 (m, 5H), 4.65-4.75 (m, 1H), 3.98-4.25 (m, 4H), $3.78(\mathrm{br} \mathrm{d}, J=$ $9.2 \mathrm{~Hz}, 1 \mathrm{H}), 3.26$ (dd, $J=13.4,4.4 \mathrm{~Hz}, 1 \mathrm{H}), 3.00(\mathrm{br} \mathrm{d}, J=3.6 \mathrm{~Hz}, 1 \mathrm{H}), 2.74$ (dd, $J=13.4,9.5 \mathrm{~Hz}, 1 \mathrm{H}$ ), 2.5-2.70 (m, 2H), 2.16 (s, 3H), 1.70-1.82 (m, 1H), 1.53-1.62 (m, 2H), 1.24 (d, $J=6.4 \mathrm{~Hz}, 3 \mathrm{H}), 1.01$ (d, $J$ $=7.0 \mathrm{~Hz}, 3 \mathrm{H})$

${ }^{13}$ C NMR $\left(100 \mathrm{MHz}, \mathrm{CDCl}_{3}\right) \delta 210.0,176.1,153.6,135.2,129.6,129.2,127.7,75.8,74.0,66.5,55.4$, $41.1,40.9,38.3,30.3,29.0,12.1,7.1$

MS (ES) calculated for $\mathrm{C}_{21} \mathrm{H}_{30} \mathrm{NO}_{6}[\mathrm{M}+\mathrm{H}]^{+} 392.2$, found 392.2

Characterization data for $(S)-2-((1 S, 3 R, 4 R, 5 S)-1,4-D i m e t h y l-2,8-d i o x a b i c y c l o[3.2 .1]$ octan-3-yl)propan-1-ol (35):

Physical state: colorless oil (94\% yield)

TLC: $R_{f}=0.50(1: 2$, hexanes/EtOAc)

Optical rotation: $[\alpha]_{D}^{20}=-11.1\left(c=0.20\right.$ in $\left.\mathrm{CHCl}_{3}\right)$

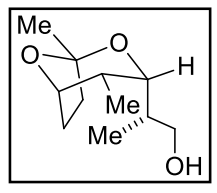

${ }^{1}$ H NMR (400 MHz, $\left.\mathrm{CDCl}_{3}\right) \delta 4.00-4.06(\mathrm{~m}, 1 \mathrm{H}), 3.56(\mathrm{br} \mathrm{dd}, J=8.7 \mathrm{~Hz}, 1 \mathrm{H}), 3.49(\mathrm{br} \mathrm{d}, J=10.2 \mathrm{~Hz}$, $1 \mathrm{H}), 3.28$ (br d, $J=10.1 \mathrm{~Hz}, 1 \mathrm{H}), 2.00-2.11(\mathrm{~m}, 2 \mathrm{H}), 1.60-1.85$ (m, 4H), 1.41 (s, 3H), 1.10 (d, $J=6.8 \mathrm{~Hz}$, $3 \mathrm{H}), 1.02(\mathrm{~d}, J=6.8 \mathrm{~Hz}, 3 \mathrm{H})$

${ }^{13}$ C NMR $\left(100 \mathrm{MHz}, \mathrm{CDCl}_{3}\right) \delta 106.8,84.1,73.2,62.1,41.9,39.9,36.9,29.6,21.4,15.2,9.7$

MS (ES) calculated for $\mathrm{C}_{11} \mathrm{H}_{20} \mathrm{O}_{3} \mathrm{Na}[\mathrm{M}+\mathrm{Na}]^{+} 223.1$, found 223.2

\section{(R)-2-((1S,3S,4R,5S)-1,4-Dimethyl-2,8-dioxabicyclo[3.2.1]octan-3-yl)propan-1-ol (S2)}

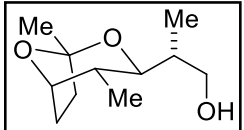

To a solution of benzyl ether $32(65 \mathrm{mg}, 0.22 \mathrm{mmol})$ in absolute EtOH $(0.5 \mathrm{~mL})$ was added $\mathrm{Pd} / \mathrm{C}(5 \%$ by weight, $15 \mathrm{mg}$ ) in one portion. The slurry was degassed, purged with $\mathrm{H}_{2}$ three times and stirred vigorously for $1 \mathrm{~h}$ at RT. The slurry was filtered through celite ${ }^{\circledR}$, eluting with EtOAc and concentrated in vacuo to give the title alcohol S2 (43 mg, 96\%) that was used without further purification. All spectral data matched those reported by Paterson [Paterson, I.; Razzak, M.; Anderson, E. A. Org. Lett. 2008, 10, 3295-3298] and material obtained from $\mathrm{LiBH}_{4}$ reduction of $\mathbf{3 3}$ as reported by us [Liu, J.; De Brabander, J. K. J. Am. Chem. Soc. 2009, 131, 12562-12563].

Physical state: colorless oil

TLC: $R_{f}=0.20$ (3:7, EtOAc/hexanes)

Optical rotation: $[\alpha]_{D}^{20}=-20.7\left(c=1.0, \mathrm{CDCl}_{3}\right)$

${ }^{1} \mathbf{H}$ NMR $\left(400 \mathrm{MHz}, \mathrm{CDCl}_{3}\right) \delta 4.22(\mathrm{dd}, J=6.9,3.6 \mathrm{~Hz}, 1 \mathrm{H}), 3.72(\mathrm{dd}, J=10.9,3.6 \mathrm{~Hz}, 1 \mathrm{H}), 3.68-3.61$ (m, 2H), $2.42(\mathrm{~m}, 1 \mathrm{H}), 2.07-1.76(\mathrm{~m}, 6 \mathrm{H}), 1.46(\mathrm{~s}, 3 \mathrm{H}), 1.00(\mathrm{~d}, J=7.1 \mathrm{~Hz}, 3 \mathrm{H}), 0.71(\mathrm{~d}, J=6.9 \mathrm{~Hz}, 3 \mathrm{H})$

${ }^{13} \mathbf{C}$ NMR $\left(100 \mathrm{MHz}, \mathrm{CDCl}_{3}\right) \delta 105.0,80.1,77.6,67.6,35.1,34.5,34.1,24.0,23.9,12.6,9.4$

MS (ES) calculated for $\mathrm{C}_{11} \mathrm{H}_{21} \mathrm{O}_{3}[\mathrm{M}+\mathrm{H}]^{+}$201.1, found 201.1 

cooled to $0{ }^{\circ} \mathrm{C}$. To the cold slurry was added the above-prepared alcohol $\mathbf{S 2}(137 \mathrm{mg}, 0.68 \mathrm{mmol})$ in $\mathrm{CH}_{2} \mathrm{Cl}_{2}(1+0.5 \mathrm{~mL})$ dropwise. The reaction was warmed to RT and stirred for $20 \mathrm{~min}$. The reaction was recooled to $0{ }^{\circ} \mathrm{C}$ and quenched by the addition of saturated aqueous $\mathrm{NaHCO}_{3}(2 \mathrm{~mL})$ and saturated aqueous $\mathrm{Na}_{2} \mathrm{~S}_{2} \mathrm{O}_{3}(4 \mathrm{~mL})$. The reaction was stirred for $1 \mathrm{~h}$ while warming to $\mathrm{RT}$. The layers were separated and the aqueous layer was extracted with $\mathrm{CH}_{2} \mathrm{Cl}_{2}(5 \mathrm{~mL} \times 4)$. The combined organic fractions were dried over $\mathrm{Na}_{2} \mathrm{SO}_{4}$, filtered and concentrated to dryness in vacuo. The resulting oil was purified by FC (silica gel, $5 \% \rightarrow 20 \%$, EtOAc/hexanes) to give aldehyde 38 (129 mg, 95\%).

Physical state: pale yellow oil

TLC: $R_{f}=0.37(3: 7$, EtOAc/hexanes)

Optical rotation: $[\alpha]_{D}^{20}=-12.5\left(c=0.4, \mathrm{CH}_{2} \mathrm{Cl}_{2}\right)$

${ }^{1}$ H NMR $\left(500 \mathrm{MHz}, \mathrm{CDCl}_{3}\right) \delta 9.66(\mathrm{~d}, J=0.7 \mathrm{~Hz}, 1 \mathrm{H}), 4.24(\mathrm{dd}, J=6.3,3.3 \mathrm{~Hz}, 1 \mathrm{H}), 3.98(\mathrm{dd}, J=10.5$, $2.3 \mathrm{~Hz}, 1 \mathrm{H}), 2.37$ (qd, $J=6.9,2.4 \mathrm{~Hz}, 1 \mathrm{H}), 2.07-1.75(\mathrm{~m}, 5 \mathrm{H}), 1.40(\mathrm{~s}, 3 \mathrm{H}), 1.14(\mathrm{~d}, J=7.1 \mathrm{~Hz}, 3 \mathrm{H}), 0.75$ $(\mathrm{d}, J=7.0 \mathrm{~Hz}, 3 \mathrm{H})$

${ }^{13} \mathbf{C}$ NMR $\left(125 \mathrm{MHz}, \mathrm{CDCl}_{3}\right) \delta 204.9,105.3,79.9,74.1,47.3,34.4,34.9,24.0,23.8,12.7,6.7$

MS (ES) calculated for $\mathrm{C}_{11} \mathrm{H}_{18} \mathrm{O}_{3} \mathrm{Na}[\mathrm{M}+\mathrm{Na}]^{+} 221.1$, found 221.1

(S)-2-((1S,3R,4R,5S)-1,4-Dimethyl-2,8-dioxabicyclo[3.2.1]octan-3-yl)pentan-3-one (15)

$\sum_{\mathrm{Me}}^{\mathrm{Me}} \prod_{\mathrm{O}}^{\mathrm{Me}}$

To a solution of 38 (99 mg, $0.5 \mathrm{mmol})$ in THF $(5 \mathrm{~mL})$, Grignard reagent EtMgBr $(0.5$ $\mathrm{mL}, 3.0 \mathrm{M}$ in ether) was added in one portion at $0{ }^{\circ} \mathrm{C}$ under an $\mathrm{Ar}$ atmosphere, which was stirred for an additional $0.5 \mathrm{~h}$. It then was quenched with water $(5 \mathrm{~mL})$, and extracted with EtOAc $(5$ $\mathrm{mL} \times 3)$. After concentration, the residue was dissolved in $\mathrm{CH}_{2} \mathrm{Cl}_{2}(5 \mathrm{~mL})$, and a slurry of Dess-Martin periodinane $(483 \mathrm{mg}, 1.0 \mathrm{mmol})$ and $\mathrm{NaHCO}_{3}(168 \mathrm{mg}, 2.0 \mathrm{mmol})$ was added. The reaction was stirred for $60 \mathrm{~min}$, and then quenched by the addition of saturated aqueous $\mathrm{NaHCO}_{3}(2 \mathrm{~mL})$ and saturated aqueous $\mathrm{Na}_{2} \mathrm{~S}_{2} \mathrm{O}_{3}(4 \mathrm{~mL})$. The layers were separated and the aqueous layer was extracted with $\mathrm{CH}_{2} \mathrm{Cl}_{2}(4$ $\times 5 \mathrm{~mL}$ ). The combined organic fractions were dried over $\mathrm{Na}_{2} \mathrm{SO}_{4}$, filtered and concentrated to dryness in vacuo. The resulting oil was purified by FC (silica gel, $5 \% \rightarrow 20 \%$, EtOAc/hexanes) to give ethyl ketone 15 (103 mg, 91\%, over two steps). All spectral data matched those of material obtained from 33 via the Weinreb route (bottom of Scheme 7) as reported by [Liu, J.; De Brabander, J. K. J. Am. Chem. Soc. 2009, $131,12562-12563]$.

Physical state: colorless oil

TLC: $R_{f}=0.60(4: 1$, hexanes/EtOAc $)$ 
Optical rotation: $[\alpha]_{D}^{20}=-7.8\left(c=1.0\right.$ in $\left.\mathrm{CH}_{2} \mathrm{Cl}_{2}\right)$

${ }^{1}$ H NMR $\left(400 \mathrm{MHz}, \mathrm{CDCl}_{3}\right) \delta: 4.20(\mathrm{dd}, J=6.0,3.6,1 \mathrm{H}), 3.84(\mathrm{dd}, J=10.4,3.2,1 \mathrm{H}), 2.54-2.46(\mathrm{~m}, 3 \mathrm{H})$, $1.98-1.76(\mathrm{~m}, 5 \mathrm{H}), 1.41(\mathrm{~s}, 3 \mathrm{H}), 1.12(\mathrm{~d}, J=7.2,3 \mathrm{H}), 1.02(\mathrm{t}, J=7.2,3 \mathrm{H}), 0.72(\mathrm{~d}, J=7.2,3 \mathrm{H})$

${ }^{13} \mathbf{C}$ NMR $\left(75 \mathrm{MHz}, \mathrm{CDCl}_{3}\right) \delta 213.6,105.2,80.1,75.6,48.1,34.8,34.4,33.9,24.2,24.0,13.0,9.5,7.9$

MS (ES) calculated for $\mathrm{C}_{13} \mathrm{H}_{23} \mathrm{O}_{3}[\mathrm{M}+\mathrm{H}]^{+} 227.2$, found 227.1

(2R,3R)-1-((4-Methoxybenzyl)oxy)-2-methylhex-5-en-3-yl methacrylate (S3)

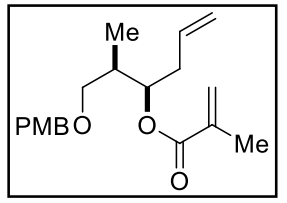

To a solution of methacrylic acid $(69 \mu \mathrm{L}, 0.8 \mathrm{mmol})$ and alcohol 41 (100 mg, $0.4 \mathrm{mmol}$, prepared according to: Liu, J.; De Brabander, J. K. J. Am. Chem. Soc. 2009, 131, $12562-$

$12563)$ in dry $\mathrm{CH}_{2} \mathrm{Cl}_{2}(10 \mathrm{~mL})$ was added DCC (412 mg, $\left.2.0 \mathrm{mmol}\right)$ and DMAP (25 mg, $0.2 \mathrm{mmol}$ ) at $0{ }^{\circ} \mathrm{C}$ under a $\mathrm{N}_{2}$ atmosphere. After stirring at $0{ }^{\circ} \mathrm{C}$ for $30 \mathrm{~min}$, the solution was warmed to RT and stirred for $12 \mathrm{~h}$, then poured into saturated aqueous $\mathrm{NaHCO}_{3}(10 \mathrm{~mL})$. The aqueous layer was extracted with $\mathrm{CH}_{2} \mathrm{Cl}_{2}(10 \mathrm{~mL} \times 3)$ and the combined organic phase was washed with brine, dried over $\mathrm{Na}_{2} \mathrm{SO}_{4}$ and concentrated in vacuo. The crude was purified by FC (silica gel, $0 \rightarrow 10 \%$, EtOAc/hexanes) to give the title compound $\mathbf{S 3}$ (114 $\mathrm{mg}, 90 \%)$.

Physical state: yellow oil

TLC: $R_{f}=0.50(5: 1$, hexanes/EtOAc)

Optical rotation: $[\alpha]_{D}^{20}=+9.3\left(c=0.6\right.$ in $\left.\mathrm{CDCl}_{3}\right)$

${ }^{1}$ H NMR (400 MHz, $\left.\mathrm{CDCl}_{3}\right) \delta 7.24(\mathrm{~d}, J=6.8 \mathrm{~Hz}, 2 \mathrm{H}), 6.86(\mathrm{~d}, J=6.8 \mathrm{~Hz}, 2 \mathrm{H}), 6.05$ (br s, 1H), 5.73 (tdd, $J=7.0,7.1,14.2,1 \mathrm{H}), 5.50(\mathrm{br} \mathrm{s}, 1 \mathrm{H}), 5.23-5.13(\mathrm{~m}, 1 \mathrm{H}), 5.05(\mathrm{~d}, J=14.2 \mathrm{~Hz}, 1 \mathrm{H}), 5.00(\mathrm{~d}, J=7.0$ $\mathrm{Hz}, 1 \mathrm{H}), 4.38$ (s, 2H), 3.79 (s, 3H), 3.31 (dd, $J=7.0,7.0 \mathrm{~Hz}, 1 \mathrm{H}), 3.25$ (dd, J=7.0, 7.0 Hz, 1H), 2.26$2.47(\mathrm{~m}, 2 \mathrm{H}), 1.98-2.11(\mathrm{~m}, 1 \mathrm{H}), 1.90(\mathrm{~s}, 3 \mathrm{H}), 0.96(\mathrm{~d}, J=6.9 \mathrm{~Hz}, 3 \mathrm{H})$

${ }^{13}$ C NMR $\left(100 \mathrm{MHz}, \mathrm{CDCl}_{3}\right) \delta 167.0,159.2,136.8,134.1,130.6,129.4,125.2,125.2,117.7,113.8,73.7$, $72.9,72.2,55.4,36.6,18.5,11.6$

MS (ES) calculated for $\mathrm{C}_{19} \mathrm{H}_{27} \mathrm{O}_{4}[\mathrm{M}+\mathrm{H}]^{+} 319.2$, found 319.2

(R)-6-((R)-1-((4-Methoxybenzyl)oxy)propan-2-yl)-3-methyl-5,6-dihydro-2H-pyran-2-one (43a)

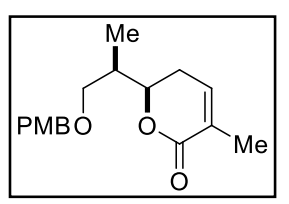
Grubbs' second generation catalyst (1,3-Bis-(2,4,6-trimethylphenyl)-2(imidazolidinylidene)-(dichlorophenylmethylene)(tricyclohexylphosphine) ruthenium, $28 \mathrm{mg}, 0.033 \mathrm{mmol})$ was added to a solution of the above-prepared diene $\mathbf{S 3}(0.33$ mmol) in dry $\mathrm{CH}_{2} \mathrm{Cl}_{2}(120 \mathrm{~mL})$. The reaction was refluxed for $14 \mathrm{~h}$ under $\mathrm{N}_{2}$. The solution was concentrated and purified by FC (silica gel, $0 \rightarrow 15 \%$, EtOAc/hexanes) to give compound 43a (62 mg, 64\%) and recovered starting material (19 mg, 20\%).

Physical state: brown oil 
TLC: $R_{f}=0.20(5: 1$, hexanes/EtOAc)

Optical rotation: $[\alpha]_{D}^{20}=+13.5\left(c=0.4\right.$ in $\left.\mathrm{CDCl}_{3}\right)$

${ }^{1}$ H NMR $\left(400 \mathrm{MHz}, \mathrm{CDCl}_{3}\right) \delta 7.22(\mathrm{~d}, J=6.8 \mathrm{~Hz}, 2 \mathrm{H}), 6.88(\mathrm{~d}, J=6.8 \mathrm{~Hz}, 2 \mathrm{H}), 6.57$ (br d, $J=5.0 \mathrm{~Hz}$, 1H), 4.51 (ddd, $J=13.2,4.0,3.6 \mathrm{~Hz}, 1 \mathrm{H}), 4.41$ (d, $J=11.2,1 \mathrm{H}), 4.37$ (d, $J=11.2,1 \mathrm{H}), 3.79$ (s, 3H), $3.50(\mathrm{dd}, J=7.2,9.2 \mathrm{~Hz}, 1 \mathrm{H}), 3.40(\mathrm{dd}, J=5.2,9.2 \mathrm{~Hz}, 1 \mathrm{H}), 2.44$ (m, 1H), 2.16 (m, 1H), 1.99 (m, 1H), $1.91(\mathrm{~s}, 3 \mathrm{H}), 1.02(\mathrm{~d}, J=7.2 \mathrm{~Hz}, 3 \mathrm{H})$

${ }^{13}$ C NMR $\left(100 \mathrm{MHz}, \mathrm{CDCl}_{3}\right) \delta 166.5,159.4,139.7,130.5,130.1,129.5,114.0,82.10,73.1,71.5,55.5$, $37.7,27.8,17.2,12.1$

MS (ES) calculated for $\mathrm{C}_{17} \mathrm{H}_{22} \mathrm{O}_{4} \mathrm{Na}[\mathrm{M}+\mathrm{Na}]^{+}, 313.1$, found 313.2

\section{(S)-2-((R)-5-Methyl-6-oxo-3, 6-dihydro-2H-pyran-2-yl)propanol (S4)}

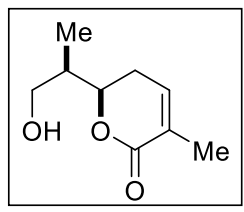

To a solution of $43 \mathbf{a}(0.14 \mathrm{mmol})$ in $\mathrm{CH}_{2} \mathrm{Cl}_{2} / \mathrm{H}_{2} \mathrm{O}(8 \mathrm{~mL} / 0.4 \mathrm{~mL})$ was added DDQ $(38 \mathrm{mg}$, $0.17 \mathrm{mmol}$ ). After stirring at RT for $1 \mathrm{~h}$, the solution was poured into saturated aqueous

$\mathrm{NaHCO}_{3}(10 \mathrm{~mL})$ and extracted with $\mathrm{CH}_{2} \mathrm{Cl}_{2}(3 \times 10 \mathrm{~mL})$, after which the combined organic phases were washed with brine, dried over $\mathrm{Na}_{2} \mathrm{SO}_{4}$ and concentrated in vacuo. The crude was purified by FC (silica gel, 10\% $\rightarrow 35 \%$, EtOAc/hexanes) to give the alcohol S4.

Physical state: pale yellow oil

TLC: $R_{f}=0.23(1: 1$, hexanes/EtOAc)

Optical rotation: $[\alpha]_{D}^{20}=+35.0\left(c=0.65\right.$ in $\left.\mathrm{CDCl}_{3}\right)$

${ }^{1}$ H NMR $\left(400 \mathrm{MHz}, \mathrm{CDCl}_{3}\right) \delta 6.62(\mathrm{~m}, 1 \mathrm{H}), 4.57(\mathrm{~m}, 1 \mathrm{H}), 3.73(\mathrm{dd}, J=10.8,7.4 \mathrm{~Hz}, 1 \mathrm{H}), 3.62(\mathrm{dd}, J=$ 10.8, $5.3 \mathrm{~Hz}, 1 \mathrm{H}), 2.48(\mathrm{~m}, 1 \mathrm{H}), 2.05-2.24(\mathrm{~m}, 2 \mathrm{H}), 1.87(\mathrm{~m}, 1 \mathrm{H}), 1.85(\mathrm{~s}, 3 \mathrm{H}), 1.01(\mathrm{~d}, J=6.8 \mathrm{~Hz}, 3 \mathrm{H})$

${ }^{13}$ C NMR $\left(100 \mathrm{MHz}, \mathrm{CDCl}_{3}\right) \delta 166.6,141.0,128.1,78.4,64.8,39.1,27.4,17.0,11.3$

MS (ES) calculated for $\mathrm{C}_{9} \mathrm{H}_{15} \mathrm{O}_{3}[\mathrm{M}+\mathrm{H}]^{+} 171.1$, found: 171.1

(S)-2-((R)-5-Methyl-6-oxo-3, 6-dihydro-2H-pyran-2-yl)propanal (14a)

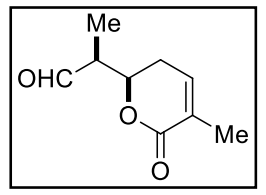

To a solution of the above-prepared alcohol $\mathbf{S 4}(80 \mathrm{mg}, 0.234 \mathrm{mmol})$ in dry $\mathrm{CH}_{2} \mathrm{Cl}_{2}(10$ $\mathrm{mL})$ was added Dess-Martin reagent $(198 \mathrm{mg}, 0.47 \mathrm{mmol})$ and solid $\mathrm{NaHCO}_{3}(79 \mathrm{mg}$, $0.94 \mathrm{mmol}$ ). The reaction was stirred for $30 \mathrm{~min}$ at $\mathrm{RT}$, then poured into aqueous $\mathrm{NaHCO}_{3}(15 \mathrm{~mL})$. The aqueous layer was extracted with $\mathrm{CH}_{2} \mathrm{Cl}_{2}(10 \mathrm{~mL} \times 3)$ and the combined organic phase was washed with brine, dried over $\mathrm{Na}_{2} \mathrm{SO}_{4}$ and concentrated in vacuo. The crude was purified by FC (silica gel, $5 \% \rightarrow 25 \%$, EtOAc/hexanes) to give compound 14a (95\%).

Physical state: colorless oil

TLC: $R_{f}=0.35(1: 1$, hexanes/EtOAc)

Optical rotation: $[\alpha]_{D}^{20}=-25.7\left(c=0.50\right.$ in $\left.\mathrm{CDCl}_{3}\right)$ 
${ }^{1}$ H NMR (500 MHz, $\left.\mathrm{CDCl}_{3}\right) \delta 9.79(\mathrm{~s}, 1 \mathrm{H}), 6.64(\mathrm{~m}, 1 \mathrm{H}), 4.78(\mathrm{ddd}, J=12.0,5.4,4.4 \mathrm{~Hz}, 1 \mathrm{H}), 2.73(\mathrm{~m}$, $1 \mathrm{H}), 2.41(\mathrm{~m}, 2 \mathrm{H}), 1.94(\mathrm{~s}, 3 \mathrm{H}), 1.25(\mathrm{~d}, J=5.7 \mathrm{~Hz}, 3 \mathrm{H})$

${ }^{13} \mathbf{C}$ NMR $\left(125 \mathrm{MHz}, \mathrm{CDCl}_{3}\right) \delta 202.0,165.4,138.9,128.6,77.0,49.7,27.5,17.1,9.2$

MS (ES) calculated for $\mathrm{C}_{9} \mathrm{H}_{13} \mathrm{O}_{3}[\mathrm{M}+\mathrm{H}]^{+} 169.1$, found 169.1

$(R)-6-((2 R, 3 S, 4 R, 6 S)-6-((1 S, 3 R, 4 R, 5 S)-1,4-D i m e t h y l-2,8-d i o x a b i-c y c l o[3.2 .1]$ octan-3-yl)-3-hydroxy4-methyl-5-oxo-heptan-2-yl)-3-methyl-5,6-dihydro-2H-pyran-2-one (44a)

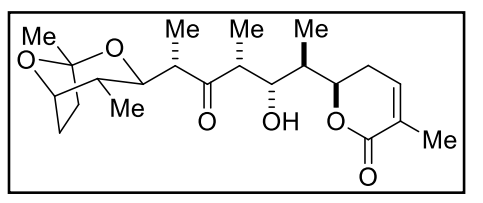
To a solution of ketone $15(56.5 \mathrm{mg}, 0.25 \mathrm{mmol})$ in dry THF $(5 \mathrm{~mL})$ at $78^{\circ} \mathrm{C}$ was added a solution of lithium bis(trimethylsilyl)amide (1 M in THF, $0.3 \mathrm{~mL}, 0.3 \mathrm{mmol})$ dropwise. The resulting yellow solution was stirred at $78{ }^{\circ} \mathrm{C}$ for $2 \mathrm{~h}$ and then a solution of aldehyde 14a $(58.8 \mathrm{mg}, 0.35 \mathrm{mmol})$ in THF $(1 \mathrm{~mL})$ was added. After stirring at $-78{ }^{\circ} \mathrm{C}$ for $2 \mathrm{~h}$, the reaction was quenched by the addition of $\mathrm{pH} 7$ phosphate buffer $(6 \mathrm{~mL})$. The aqueous layer was extracted with ether $(10 \mathrm{~mL} \times 3)$ and the combined organic phase was washed with brine, dried over $\mathrm{MgSO}_{4}$ and concentrated in vacuo. The crude was purified by FC (silica gel, $5 \% \rightarrow 25 \%$, EtOAc/hexanes) to give compound 44a (69 mg, 70\%).

Physical state: colorless oil

TLC: $R_{f}=0.20(4: 1$, hexanes/EtOAc)

Optical rotation: $[\alpha]_{D}^{20}=-14.2\left(c=0.27\right.$ in $\left.\mathrm{CH}_{2} \mathrm{Cl}_{2}\right)$

${ }^{1} \mathbf{H}$ NMR $\left(400 \mathrm{MHz}, \mathrm{CDCl}_{3}\right) \delta 6.60(\mathrm{~m}, 1 \mathrm{H}), 4.96(\mathrm{ddd}, J=13.4,3.6,1.7 \mathrm{~Hz}, 1 \mathrm{H}), 4.20(\mathrm{dd}, J=6.2,3.4$ $\mathrm{Hz}, 1 \mathrm{H}), 4.01(\mathrm{~d}, J=9.9 \mathrm{~Hz}, 1 \mathrm{H}), 3.82(\mathrm{dd}, J=10.3,3.1 \mathrm{~Hz}, 1 \mathrm{H}), 3.54(\mathrm{~d}, J=2.0 \mathrm{~Hz}, 1 \mathrm{H}), 3.03(\mathrm{dq}, J=$ 7.0, $1.5 \mathrm{~Hz}, 1 \mathrm{H}), 2.77(\mathrm{dq}, J=7.0,3.1 \mathrm{~Hz}, 1 \mathrm{H}), 2.44-2.54(\mathrm{~m}, 1 \mathrm{H}), 2.00-2.10(\mathrm{~m}, 1 \mathrm{H}), 1.86(\mathrm{~s}, 3 \mathrm{H}), 1.50-$ $2.00(\mathrm{~m}, 6 \mathrm{H}), 1.42(\mathrm{~s}, 3 \mathrm{H}), 1.12(\mathrm{~d}, J=7.2 \mathrm{~Hz}, 3 \mathrm{H}), 1.06(\mathrm{~d}, J=7.2 \mathrm{~Hz}, 3 \mathrm{H}), 0.94(\mathrm{~d}, J=7.2 \mathrm{~Hz}, 3 \mathrm{H})$, $0.77(\mathrm{~d}, J=6.8 \mathrm{~Hz}, 3 \mathrm{H})$

${ }^{13}$ C NMR $\left(100 \mathrm{MHz}, \mathrm{CDCl}_{3}\right) \delta 218.6,166.5,139.7,128.5,105.5,80.1,76.6,75.8,69.8,47.3,43.4,39.2$, $34.8,34.4,28.1,24.03,24.00,17.6,13.1,9.9,8.9$

IR (film): 326, 2937, 1778, 1693, 1386, 1211, $972 \mathrm{~cm}^{-1}$

MS (ES) calculated for $\mathrm{C}_{22} \mathrm{H}_{35} \mathrm{O}_{6}[\mathrm{M}+\mathrm{H}]^{+} 395.2$, found 395.2

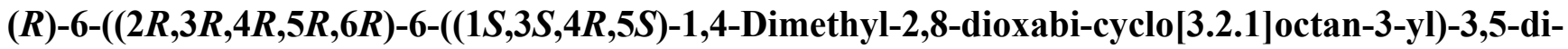
hydroxy-4-methylheptan-2-yl)-3-methyl-5,6-dihydro-2H-pyran-2-one (S5)

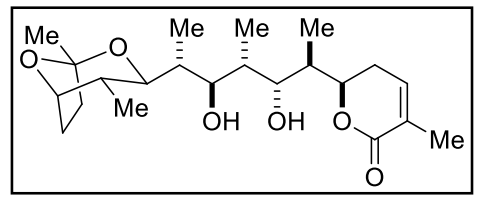

Tetramethylammonium triacetoxyborohydride $(345 \mathrm{mg}, 1.31 \mathrm{mmol})$ was added to $\mathrm{CH}_{3} \mathrm{CN} /$ acetic acid $(3 \mathrm{~mL} / 3 \mathrm{~mL})$, and the resulting solution was stirred for $30 \mathrm{~min}$ at $\mathrm{RT}$ and cooled to $-20{ }^{\circ} \mathrm{C}$ before ketone $44 \mathrm{a}(65 \mathrm{mg}$, $0.164 \mathrm{mmol}$ ) was added. After $48 \mathrm{~h}$ at $-20^{\circ} \mathrm{C}$, the reaction was quenched by the addition of $20 \mathrm{~mL}$ of a 
saturated aqueous solution of Rochelle's salt. The aqueous layer was then extracted with $\mathrm{CH}_{2} \mathrm{Cl}_{2}(20 \mathrm{~mL}$ $\times 3$ ). The combined organic layers were washed with saturated aqueous $\mathrm{NaHCO}_{3}$ and brine, dried over $\mathrm{Na}_{2} \mathrm{SO}_{4}$ and concentrated in vacuo. The residue was purified by FC (silica gel, $10 \% \rightarrow 35 \%$, EtOAc/hexanes) to give the anti-diol diol S5 (56 mg, 86\%, contaminated with $\sim 5-10 \%$ of the syn-diol). We have not attempted to separate the diastereomers and data reported below are for the mixture.

Physical state: colorless oil

TLC: $R_{f}=0.15(4: 1$, hexanes/EtOAc)

Optical rotation: $[\alpha]_{D}^{20}=-22.1\left(c=0.2\right.$ in $\left.\mathrm{CDCl}_{3}\right)$

${ }^{1}$ H NMR $\left(600 \mathrm{MHz}, \mathrm{CDCl}_{3}\right) \delta 6.62(\mathrm{dt}, J=6.5,1.9 \mathrm{~Hz}, 1 \mathrm{H}), 5.03-4.94(\mathrm{~m}, 1 \mathrm{H}), 4.23(\mathrm{dd}, J=6.6,3.3 \mathrm{~Hz}$, $1 \mathrm{H}), 4.13(\mathrm{~d}, J=10.1 \mathrm{~Hz}, 1 \mathrm{H}), 3.85-3.75(\mathrm{~m}, 1 \mathrm{H}), 3.66-3.57(\mathrm{~m}, 1 \mathrm{H}), 3.41(\mathrm{~s}, 1 \mathrm{H}), 3.34(\mathrm{~d}, J=5.8 \mathrm{~Hz}$, 1H), 2.54 (ddt, $J=18.1,13.4,2.5 \mathrm{~Hz}, 1 \mathrm{H}), 2.17$ (s, 1H), 2.14-2.03 (m, 1H), 2.00-1.84 (m, 8H), 1.84-1.77 $(\mathrm{m}, 1 \mathrm{H}), 1.46(\mathrm{~s}, 3 \mathrm{H}), 0.98(\mathrm{~d}, J=6.9 \mathrm{~Hz}, 3 \mathrm{H}), 0.96(\mathrm{~d}, J=7.2 \mathrm{~Hz}, 3 \mathrm{H}), 0.92(\mathrm{dd}, J=8.5,6.9 \mathrm{~Hz}, 3 \mathrm{H})$, $0.75(\mathrm{~d}, J=6.9 \mathrm{~Hz}, 3 \mathrm{H})$

${ }^{13}$ C NMR $\left(150 \mathrm{MHz}, \mathrm{CDCl}_{3}\right) \delta 166.7,139.8,128.4,105.3,80.3,79.0,76.8,75.6,70.1,39.9,35.6,35.5$, $34.6,33.9,28.1,24.2,24.1,17.3,13.0,11.9,10.6,10.0$

MS (ES) calculated for $\mathrm{C}_{22} \mathrm{H}_{36} \mathrm{O}_{6} \mathrm{Na}[\mathrm{M}+\mathrm{Na}]^{+} 419.2$, found 419.2

$(R)-6-((S)-1-((4 S, 5 R, 6 R)-6-((R)-1-((1 S, 3 R, 4 R, 5 S)-1,4-D i m e t h y l-2,8-d i o x a-b i c y c l o[3.2 .1]$ octan-3-yl)ethyl)-2,2,5-trimethyl-1,3-dioxan-4-yl)ethyl)-3-methyl-5,6-dihydro-2H-pyran-2-one (45a)

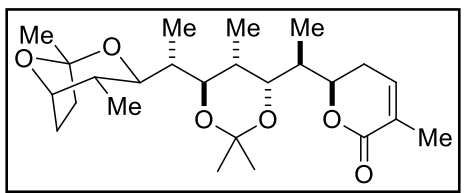

To a solution of anti-diol S5 $(20 \mathrm{mg}, 0.05 \mathrm{mmol})$ in 2,2-dimethoxypropane/acetone $(1.0 \mathrm{~mL} / 1.0 \mathrm{~mL})$ was added $2 \mathrm{mg}$ PPTS. The mixture was stirred at room temperature for $30 \mathrm{~min}$ and concentrated in vacuo. The crude was purified by FC (silica gel, $2 \% \rightarrow 15 \%$, EtOAc/hexanes) to give compound $45 \mathbf{a}$ (19 $\mathrm{mg}, 87 \%$ ).

Physical state: colorless oil

TLC: $R_{f}=0.75(4: 1$, hexanes/EtOAc)

Optical rotation: $[\alpha]_{D}^{20}=-13.5(c=0.1$ in $\mathrm{MeOH})$

${ }^{1}$ H NMR (400 MHz, CD 3 OD) $\delta 6.60(\mathrm{dd}, J=6.2,2.0 \mathrm{~Hz}, 1 \mathrm{H}), 4.79(\mathrm{~m}, 1 \mathrm{H}), 4.19(\mathrm{~m}, 1 \mathrm{H}), 3.95$ (dd, $J=$ 10.9, 3.6 Hz, 1H), 3.73 (dd, $J=10.6,2.0 \mathrm{~Hz}, 1 \mathrm{H}), 3.27$ (m, 1H), 2.50 (m, 1H), 2.03 (m, 1H), 1.90 (s, 3H), 1.56-1.98 (m, 8H), $1.38(\mathrm{~s}, 3 \mathrm{H}), 1.30(\mathrm{~s}, 3 \mathrm{H}), 1.24(\mathrm{~s}, 3 \mathrm{H}), 0.91(\mathrm{~d}, J=6.8 \mathrm{~Hz}, 3 \mathrm{H}), 0.88(\mathrm{~d}, J=6.8 \mathrm{~Hz}$, $3 \mathrm{H}), 0.86(\mathrm{~d}, J=6.9 \mathrm{~Hz}, 3 \mathrm{H}), 0.67(\mathrm{~d}, J=6.9 \mathrm{~Hz}, 3 \mathrm{H})$

${ }^{13}$ C NMR $\left(100 \mathrm{MHz}, \mathrm{CDCl}_{3}\right) \delta 166.5,139.9,128.4,105.0, \mathbf{1 0 0 . 8}, 80.5,76.4,75.1,73.0,68.7,39.1,36.8$, $36.3,34.4,34.0,29.9,29.5,27.8, \mathbf{2 6 . 2}, \mathbf{2 4 . 4}, 23.7,17.3,12.7,9.0,8.1$

MS (ES) calculated for $\mathrm{C}_{25} \mathrm{H}_{41} \mathrm{O}_{6}[\mathrm{M}+\mathrm{H}]^{+} 437.3$, found 437.3 
$(S, 2 Z, 4 E)-6-((4 S, 5 R, 6 R)-6-((R)-1-((1 S, 3 R, 4 R, 5 S)-1,4-D i m e t h y l-2,8-d i o x a-b i c y c l o[3.2 .1]$ octan-3-yl)ethyl)-2,2,5-trimethyl-1,3-dioxan-4-yl)-2-methylhepta-2,4-dienoic acid (S6; aka 79)

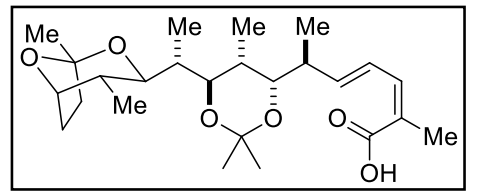

To a solution of acetonide $45 \mathbf{a}(19 \mathrm{mg}, 0.0435 \mathrm{mmol})$ in THF $(0.5 \mathrm{~mL})$, was added LiHMDS $\left(0.22 \mathrm{~mL}, 1.0 \mathrm{M}\right.$ in THF) in one portion at $0{ }^{\circ} \mathrm{C}$. After stirring for $10 \mathrm{~min}$ at $0{ }^{\circ} \mathrm{C}$, it was quenched with water $(0.5 \mathrm{~mL})$. The aqueous was extracted with EtOAc $(1 \mathrm{~mL} \times 3)$. The combined organic phase was dried over $\mathrm{MgSO}_{4}$ and the solvent was removed in vacuo. The crude residue was purified by FC (silica gel, $10 \% \rightarrow 60 \%$, EtOAc/hexanes) to give the title dienoic acid S6 (aka 79 in scheme 16; $18 \mathrm{mg}, 95 \%$ ).

Physical state: pale yellow oil

TLC: $R_{f}=0.45(1: 1$, hexanes/EtOAc)

Optical rotation: $[\alpha]_{D}^{20}=-7.2\left(c=0.10\right.$ in $\left.\mathrm{CD}_{3} \mathrm{OD}\right)$

${ }^{1}$ H NMR $\left(400 \mathrm{MHz}, \mathrm{CDCl}_{3}\right) \delta 7.21(\mathrm{ddd}, J=15.3,11.0,2.4 \mathrm{~Hz}, 1 \mathrm{H}), 6.51(\mathrm{~d}, J=11.1 \mathrm{~Hz}, 1 \mathrm{H}), 6.00$ (dd, $J=15.3,7.0 \mathrm{~Hz}, 1 \mathrm{H}), 4.19(\mathrm{~m}, 1 \mathrm{H}), 3.70(\mathrm{br} \mathrm{d}, J=10.5 \mathrm{~Hz}, 1 \mathrm{H}), 3.50(\mathrm{~m}, 1 \mathrm{H}), 3.28(\mathrm{dd}, J=9.1,6.5 \mathrm{~Hz}$, 1H), $2.41(\mathrm{~m}, 1 \mathrm{H}), 1.95(\mathrm{~s}, 3 \mathrm{H}), 1.56-1.98(\mathrm{~m}, 7 \mathrm{H}), 1.40(\mathrm{~s}, 3 \mathrm{H}), 1.25(\mathrm{~s}, 3 \mathrm{H}), 1.24(\mathrm{~s}, 3 \mathrm{H}), 0.95(\mathrm{~d}, J=$ $6.4 \mathrm{~Hz}, 3 \mathrm{H}), 0.90(\mathrm{~d}, J=6.6 \mathrm{~Hz}, 3 \mathrm{H}), 0.87(\mathrm{~d}, J=6.9 \mathrm{~Hz}, 3 \mathrm{H}), 0.66(\mathrm{~d}, J=6.6 \mathrm{~Hz}, 3 \mathrm{H})$

${ }^{13}$ C NMR $\left(100 \mathrm{MHz}, \mathrm{CDCl}_{3}\right) \delta 172.6,147.0,143.7,126.9,122.7,104.8,100.4,80.2,74.6,73.3,72.8$, $44.9,38.8,36.4,34.1,33.7,25.6,24.2,24.0,23.4,20.5,15.8,12.8,12.4,7.9$

MS (ES) calculated for $\mathrm{C}_{25} \mathrm{H}_{41} \mathrm{O}_{6}[\mathrm{M}+\mathrm{H}]^{+} 437.3$, found 437.3

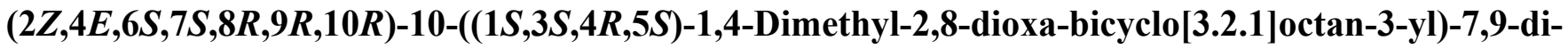
hydroxy-2,6,8-trimethylundeca-2,4-dienamide (saliniketal A (1a))

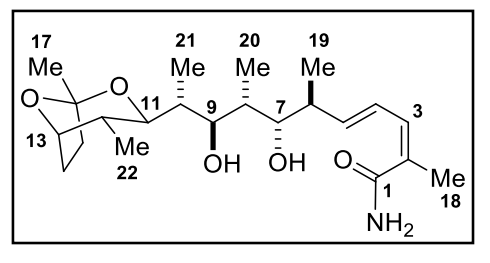

To the solution of the above-prepared dienoic acid S6 (aka 79, $9.7 \mathrm{mg}$, $0.024 \mathrm{mmol})$ in THF $(0.2 \mathrm{~mL})$ was added HOBt $(7.3 \mathrm{mg}, 0.054 \mathrm{mmol})$ and EDC (10.4 mg, $0.054 \mathrm{mmol})$ followed by ammonia (0.5 $\mathrm{M}$ in 1,4-dioxane,

$108 \mu \mathrm{L}, 0.054 \mathrm{mmol})$. After stirring the mixture at RT for $10 \mathrm{~h}$, it was

filtered over Celite. The Celite was washed twice with THF and the combined organic phase was concentrated in vacuo. The crude residue (compound $\mathbf{8 0}$ in scheme 16) was dissolved in $\mathrm{MeOH}(0.5 \mathrm{~mL})$, followed by the addition of Dowex $(5 \mathrm{mg})$. The mixture was stirred for $6 \mathrm{~h}$, filtered and purified by FC (silica gel, $0 \rightarrow 10 \%, \mathrm{MeOH} / \mathrm{CH}_{2} \mathrm{Cl}_{2}$ ) to give saliniketal A (1a, $8.4 \mathrm{mg}, 90 \%$ ). All spectral details match those reported by Fenical [Williams, P. G.; Asolkar, R. N.; Kondratyuk, T.; Pezzuto, J. M.; Jensen, P. R.; Fenical, W. J. Nat. Prod. 2007, 70, 83-88] and Paterson [Paterson, I.; Razzak, M.; Anderson, E. A. Org. Lett. 2008, 10, 3295-3298].

Physical state: white amorphous solid

TLC: $R_{f}=0.42\left(1: 10, \mathrm{MeOH} / \mathrm{CH}_{2} \mathrm{Cl}_{2}\right)$ 
Optical rotation: $[\alpha]_{D}^{20}=-7.2\left(c=0.10\right.$ in $\left.\mathrm{CD}_{3} \mathrm{OD}\right)$

${ }^{1} \mathbf{H}$ NMR (400 MHz, CD $\left.{ }_{3} \mathrm{OD}\right): \delta 6.60(\mathrm{dd}, J=15.2,11.1 \mathrm{~Hz}, 1 \mathrm{H}), 6.18(\mathrm{dd}, J=11.2,1.8 \mathrm{~Hz}, 1 \mathrm{H}), 5.79$ (dd, $J=15.0,8.3 \mathrm{~Hz}, 1 \mathrm{H}), 4.24$ (dd, $J=6.4,3.5 \mathrm{~Hz}, 1 \mathrm{H}), 3.97$ (br d, $J=10.5 \mathrm{~Hz}, 1 \mathrm{H}) ; 3.73$ (dd, $J=9.3$, $1.8 \mathrm{~Hz}, 1 \mathrm{H}) ; 3.53$ (dd, $J=8.6,4.1 \mathrm{~Hz}, 1 \mathrm{H}), 2.36$ (br ddq, $J=8.3,8.3,7.0 \mathrm{~Hz}, 1 \mathrm{H}), 1.80-2.10$ (m, 7H), 1.94 (br S, 3H), 1.40 (s, 3H), 1.02 (d, $J=7.2 \mathrm{~Hz}, 3 \mathrm{H}), 0.96$ (d, $J=6.8 \mathrm{~Hz}, 3 \mathrm{H}), 0.88$ (d, $J=7.1 \mathrm{~Hz}, 3 \mathrm{H})$, $0.74(\mathrm{~d}, J=7.1,3 \mathrm{H})$

${ }^{13}$ C NMR (100 MHz, $\left.\mathrm{CD}_{3} \mathrm{OD}\right) \delta 175.1,142.0,134.0,131.4,128.3,106.6,81.6,78.1,75.7,74.9,42.3$, $37.1,35.8,35.2,35.1,24.9,24.3,21.0,17.1,12.8,11.1,10.3$

MS (ES) calculated for $\mathrm{C}_{22} \mathrm{H}_{38} \mathrm{NO}_{5}[\mathrm{M}+\mathrm{H}]^{+} 396.3$, found 396.3

Table S3. Comparison of ${ }^{1} \mathrm{H}$ NMR for natural (500 MHz, $\left.\mathrm{CD}_{3} \mathrm{OD}\right)$ and synthetic saliniketal A (1a, 400 $\left.\mathrm{MHz}, \mathrm{CD}_{3} \mathrm{OD}\right)$

\begin{tabular}{|c|c|c|}
\hline \multirow{2}{*}{ No. } & Natural & Synthetic \\
\hline & \multicolumn{2}{|c|}{$[\delta$ in ppm, multiplicity $(J$ in $\mathrm{Hz})]$} \\
\hline 3 & 6.17, br d $(11.1,1.2)$ & $6.18, \mathrm{~d}(11.2,1.8)$ \\
\hline 4 & $6.60, \mathrm{dd}(15.3,11.1)$ & $6.60, \mathrm{dd}(15.2,11.1)$ \\
\hline 5 & $5.78, \mathrm{dd}(15.3,8.4)$ & 5.79, dd $(15.0,8.3)$ \\
\hline 6 & $2.35, \mathrm{~m}(9.3,8.4,6.8)$ & 2.36 , br ddq, $(8.3,8.3,7.0)$ \\
\hline 7 & $3.71, \mathrm{dd}(9.3,1.8)$ & $3.73, \mathrm{dd}(9.3,1.8)$ \\
\hline 8 & $1.88, \mathrm{~m}(7.4,4.9,1.8)$ & $1.80-2.10, \mathrm{~m}(1 \mathrm{H}$ of $7 \mathrm{H})$ \\
\hline 9 & $3.52, \mathrm{dd}(8.3,4.9)$ & $3.53, \mathrm{dd}(8.6,4.1)$ \\
\hline 10 & 1.84 , br dq $(8.3,7.2,1.4)$ & $1.80-2.10, \mathrm{~m}(1 \mathrm{H}$ of $7 \mathrm{H})$ \\
\hline 11 & 3.97, br d $(10.8,1.4)$ & 3.97, br d (10.5) \\
\hline 12 & 2.00, dqd $(10.8,7.3,3.4)$ & $1.80-2.10, \mathrm{~m}(1 \mathrm{H}$ of $7 \mathrm{H})$ \\
\hline 13 & 4.23 , br dd $(6.3,3.4)$ & $4.24, \mathrm{dd}(6.4,3.5)$ \\
\hline $14 \mathrm{a}$ & $1.94, \mathrm{~m}$ & $1.80-2.10, \mathrm{~m}(1 \mathrm{H}$ of $7 \mathrm{H})$ \\
\hline $14 \mathrm{~b}$ & $1.90, \mathrm{~m}$ & $1.80-2.10, \mathrm{~m}(1 \mathrm{H}$ of $7 \mathrm{H})$ \\
\hline 15 & $2.05, \mathrm{~m}$ & $1.80-2.10, \mathrm{~m}(1 \mathrm{H}$ of $7 \mathrm{H})$ \\
\hline $15 b$ & $1.80, \mathrm{~m}$ & $1.80-2.10, \mathrm{~m}(1 \mathrm{H}$ of $7 \mathrm{H})$ \\
\hline 17 & $1.39, \mathrm{~s}$ & $1.40,(\mathrm{~s})$ \\
\hline 18 & $1.94, \mathrm{~d}(1.2)$ & 1.94, br s \\
\hline 19 & $0.96, \mathrm{~d}(6.8)$ & $0.96, \mathrm{~d}(6.8)$ \\
\hline 20 & $1.02, \mathrm{~d}(7.3)$ & $1.02, \mathrm{~d}(7.2)$ \\
\hline 21 & $0.89, \mathrm{~d}(7.2)$ & $0.88, \mathrm{~d}(7.1)$ \\
\hline 22 & $0.76, \mathrm{~d}(7.3)$ & $0.74, \mathrm{~d}(7.1)$ \\
\hline
\end{tabular}


Table S4. Comparison of ${ }^{13} \mathrm{C}$ NMR for natural (125 MHz, $\left.\mathrm{CD}_{3} \mathrm{OD}\right)$ and synthetic saliniketal A (1a, 100 $\left.\mathrm{MHz}, \mathrm{CD}_{3} \mathrm{OD}\right)$

\begin{tabular}{|c|c|c|}
\hline No. & Natural $(\delta, \mathrm{ppm})$ & Synthetic $(\delta, \mathrm{ppm})$ \\
\hline 1 & 175.1 & 175.1 \\
\hline 2 & 131.4 & 131.4 \\
\hline 3 & 134.1 & 134.0 \\
\hline 4 & 128.3 & 128.3 \\
\hline 5 & 142.0 & 142.0 \\
\hline 6 & 42.3 & 42.3 \\
\hline 7 & 75.8 & 75.7 \\
\hline 8 & 35.7 & 35.8 \\
\hline 9 & 78.2 & 78.1 \\
\hline 10 & 37.1 & 37.1 \\
\hline 11 & 74.9 & 74.9 \\
\hline 12 & 35.2 & 35.2 \\
\hline 13 & 81.6 & 81.6 \\
\hline $14 \mathrm{a}$ & 24.9 & 24.9 \\
\hline 15 & 35.1 & 35.1 \\
\hline 16 & 106.4 & 106.6 \\
\hline 17 & 24.2 & 24.3 \\
\hline 18 & 20.9 & 21.0 \\
\hline 19 & 17.1 & 17.1 \\
\hline 20 & 11.1 & 11.1 \\
\hline 21 & 10.2 & 10.3 \\
\hline 22 & 12.8 & 12.8 \\
\hline
\end{tabular}

\section{2,6-Dibromocyclohexa-2,5-diene-1,4-dione (47)}

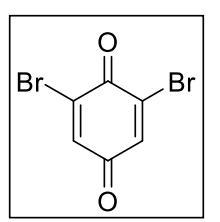

This compound was prepared according to the literature [Omura, K. Synthesis 1998, 8, 1145] with modifications. A solution of the 2,4,6-tribromophenol (2.65 g, $8 \mathrm{mmol})$ in $\mathrm{AcOH}(30 \mathrm{~mL})$ was added dropwise over a period of $5 \mathrm{~min}$ to a stirred mixture of $\mathrm{PbO}_{2}$ $(3.82 \mathrm{~g}, 16 \mathrm{mmol}), 60 \% \mathrm{HClO}_{4}(10 \mathrm{~mL})$ and $\mathrm{AcOH}(30 \mathrm{~mL})$, and the resulting mixture was stirred for $10 \mathrm{~min}$. The reaction mixture was worked up with water $(20 \mathrm{ml})$, hexanes $(20 \mathrm{~mL})$ and $\mathrm{Et}_{2} \mathrm{O}$ $(20 \mathrm{~mL})$. After extraction with hexanes and $\mathrm{Et}_{2} \mathrm{O}(v / v 1: 1,20 \mathrm{~mL} \times 3)$. The combined organic phase was dried over $\mathrm{Na}_{2} \mathrm{SO}_{4}$ and concentrated in vacuo. The residue was purified by FC (silica gel, $0 \rightarrow 15 \%$ EtOAc in hexanes) to give pure compound 47 (1.78 g, 85\%). All spectral data matched those reported by: Omura, K. Synthesis 1998, 8, 1145. 
Physical state: red solid

TLC: $R_{f}=0.50(1: 4$, EtOAc/hexanes $)$

${ }^{1} \mathbf{H}$ NMR $\left(600 \mathrm{MHz}, \mathrm{CDCl}_{3}\right) \delta 7.33(\mathrm{~s}, 2 \mathrm{H})$

${ }^{13}$ C NMR $\left(150 \mathrm{MHz}, \mathrm{CDCl}_{3}\right) \delta 182.5,172.5,138.4,135.8$

MS (ES) calculated for $\mathrm{C}_{6} \mathrm{H}_{3} \mathrm{Br}_{2} \mathrm{O}_{2}[\mathrm{M}+\mathrm{H}]^{+} 264.9$, found 264.9

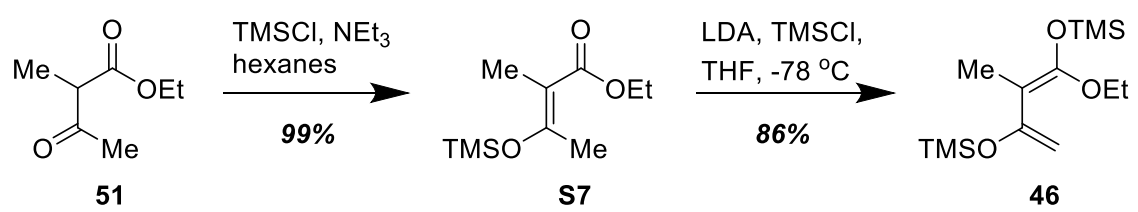

Ethyl (E)-2-methyl-3-((trimethylsilyl)oxy)but-2-enoate (S7)

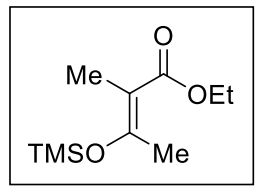

The compounds S7 and $\mathbf{4 6}$ were synthesized according to the literature: Langer, P.; Schneider, T.; Stoll, M. Chem. Eur. J. 2000, 6, 3204. To a solution of ethyl 2-methyl-3oxobutanoate $51(1.44 \mathrm{~g}, 10.0 \mathrm{mmol})$ in hexanes $(30 \mathrm{~mL})$ was added $\mathrm{NEt}_{3}(2.09 \mathrm{~mL}$,

$15.0 \mathrm{mmol})$ followed by TMSCl $(1.26 \mathrm{~mL}, 15.0 \mathrm{mmol})$. After stirring for $24 \mathrm{~h}$ at ambient temperature, the white mixture was filtered over Celite ${ }^{\circledR}$ and washed with hexanes $(20 \mathrm{~mL} \times 3)$. The combined organic phase was concentrated in vacuo. The crude residue S7 (2.14 g, 99\%) was used for the next step without further purification. All spectral data matched those reported by: Langer, P.; Schneider, T.; Stoll, M. Chem. Eur. J. 2000, 6, 3204.

Physical state: slight yellow oil

TLC: $R_{f}=0.25(1: 4$, EtOAc/hexanes)

${ }^{1} \mathbf{H}$ NMR $\left(600 \mathrm{MHz}, \mathrm{CDCl}_{3}\right) \delta 4.15(\mathrm{q}, J=7.2 \mathrm{~Hz}, 2 \mathrm{H}), 2.26(\mathrm{q}, J=1.2 \mathrm{~Hz}, 3 \mathrm{H}), 1.76(\mathrm{q}, J=1.2 \mathrm{~Hz}, 3 \mathrm{H})$, $1.28(\mathrm{t}, J=7.2 \mathrm{~Hz}, 3 \mathrm{H}), 0.24(\mathrm{~s}, 9 \mathrm{H})$

${ }^{13}$ C NMR (150 MHz, $\left.\mathrm{CDCl}_{3}\right) \delta 169.9,161.3,109.2,59.8,21.7,14.5,12.6,1.0$

MS (ES) calculated for $\mathrm{C}_{10} \mathrm{H}_{21} \mathrm{O}_{3} \mathrm{Si}[\mathrm{M}+\mathrm{H}]^{+} 217.1$, found 217.1

\section{(E)-4-Ethoxy-2,2,5,8,8-pentamethyl-6-methylene-3,7-dioxa-2,8-disilan-on-4-ene (46)}

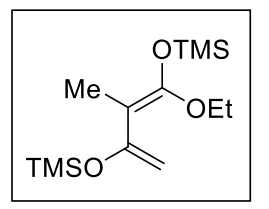

To a solution of diisopropylamine $(1.54 \mathrm{~mL}, 10.89 \mathrm{mmol})$ in THF $(30 \mathrm{~mL})$ was added $n$ -

BuLi (2.5 $\mathrm{M}$ in hexanes, $4.75 \mathrm{ml}, 11.88 \mathrm{mmol})$ at $-78{ }^{\circ} \mathrm{C}$. After stirring for $10 \mathrm{~min}$, the resulting solution was warmed to $0{ }^{\circ} \mathrm{C}$ and stirred for an additional 15 minutes at that temperature; then it was cooled back to $-78{ }^{\circ} \mathrm{C}$. A solution of the compound $\mathbf{S} 7(2.14 \mathrm{~g}, 9.89 \mathrm{mmol})$ in THF $(10 \mathrm{ml})$ was added dropwise over 10 minutes. After stirring for 15 minutes at $-78{ }^{\circ} \mathrm{C}$, $\mathrm{TMSCl}(1.88$ $\mathrm{ml}$ ) was added in one portion and stirred for an additional $15 \mathrm{~min}$. The resulting yellow mixture was warmed to RT over a $1 \mathrm{~h}$ period. After filtering over Celite ${ }^{\circledR}$ (wash with hexanes, $20 \mathrm{~mL} \times 3$ ), the 
combined organic phase was concentrated in vacuo. The crude residue (46) was used in the next step without further purification.

\section{2-Bromo-6,8-dihydroxy-7-methylnaphthalene-1,4-dione (53)}

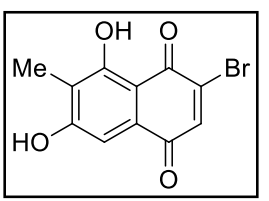

To a solution of $47(2.24 \mathrm{~g}, 8.50 \mathrm{mmol})$ in benzene $(15 \mathrm{~mL})$ was added compound 46 (crude residue prepared above) in benzene $(20 \mathrm{~mL})$ dropwise over 10 min under an $\mathrm{Ar}$ atmosphere. The resulting green solution was stirred for $4 \mathrm{~h}$ at RT and silica gel $(5 \mathrm{~g})$ was added. Then the mixture was stirred for $10 \mathrm{~h}$ at RT, followed by filtration over a plug of cotton. The filtrate was concentrated in vacuo, and the residue purified by FC (silica gel; $5 \% \rightarrow 20 \%$, EtOAc/hexanes) to give pure compound $53(1.73 \mathrm{~g}, 72 \%)$.

Physical state: red needle solid

TLC: $R_{f}=0.3(4: 1$, hexanes/EtOAc)

${ }^{1}$ H NMR (400 MHz, CD 3 OD) $\delta: 7.34(\mathrm{~s}, 1 \mathrm{H}), 6.96(\mathrm{~s}, 1 \mathrm{H}), 2.07(\mathrm{~s}, 3 \mathrm{H})$

${ }^{13}$ C NMR (100 MHz, CD 3 OD) $\delta: 182.5,180.7,162.5,162.3,140.0,139.8,131.0,119.8,110.0,108.1,7.9$

MS (ES) calculated for $\mathrm{C}_{11} \mathrm{H}_{8} \mathrm{BrO}_{4}[\mathrm{M}+\mathrm{H}]^{+} 283.0$, found 283.0

\section{2-Bromo-6,8-bis(methoxymethoxy)-7-methylnaphthalene-1,4-dione (54)}

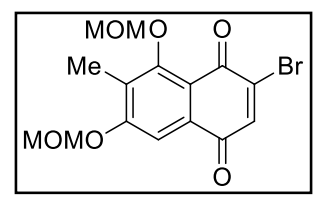

To a solution of compound $53(1.73 \mathrm{~g}, 6.1 \mathrm{mmol})$ in dry $\mathrm{CH}_{2} \mathrm{Cl}_{2}(45 \mathrm{~mL})$ was added MOMCl (1.39 mL, $18.3 \mathrm{mmol})$ and ${ }^{i} \operatorname{Pr}_{2} \mathrm{NEt}(4.25 \mathrm{~mL}, 24.4 \mathrm{mmol})$ under an $\mathrm{Ar}$ atmosphere. The resulting dark green solution was allowed to stir for $24 \mathrm{~h}$ at RT,

followed by the addition of saturated aqueous $\mathrm{NaHCO}_{3}(100 \mathrm{~mL})$. The aqueous phase was extracted with EtOAc $(50 \mathrm{~mL} \times 3)$ and the combined organic phase was dried over $\mathrm{MgSO}_{4}$ and concentrated. The residue was purified by FC (silica gel; $0 \rightarrow 15 \%$, EtOAc/hexanes) to give pure compound 54 (1.73 g, 76\%).

Physical state: dark red solid

TLC: $R_{f}=0.50(4: 1$, hexanes/EtOAc)

${ }^{1}$ H NMR (400 MHz, $\left.\mathrm{CDCl}_{3}\right) \delta 7.57(\mathrm{~s}, 1 \mathrm{H}), 7.41(\mathrm{~s}, 1 \mathrm{H}), 5.34(\mathrm{~s}, 2 \mathrm{H}), 5.09$ (s, 2H), 3.63 (s, 3H), 3.49 (s, $3 \mathrm{H}), 2.30(\mathrm{~s}, 3 \mathrm{H})$

${ }^{13}$ C NMR (100 MHz, $\left.\mathrm{CDCl}_{3}\right): \delta 182.2,176.0,160.6,158.7,142.4,138.7,132.7,129.8,117.2,108.3$, $102.0,94.5,58.1,56.9,10.5$

MS (ES) calculated for $\mathrm{C}_{15} \mathrm{H}_{16} \mathrm{BrO}_{6}[\mathrm{M}+\mathrm{H}]^{+} 371.0$, found 371.0

\section{2-Azido-6,8-bis(methoxymethoxy)-7-methylnaphthalene-1,4-dione (S8)}

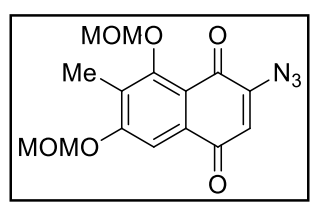

To a solution of compound 54 (200 mg, $0.54 \mathrm{mmol})$ in $\mathrm{CH}_{2} \mathrm{Cl}_{2} / \mathrm{H}_{2} \mathrm{O} / \mathrm{MeOH}(6 \mathrm{~mL} / 0.6$ $\mathrm{mL} / 0.6 \mathrm{~mL})$ was added $\mathrm{NaN}_{3}(38 \mathrm{mg}, 0.59 \mathrm{mmol})$. After stirring for $16 \mathrm{~h}$ at $\mathrm{RT}$, the reaction was quenched by the addition of saturated aqueous $\mathrm{NaHCO}_{3}$. The aqueous 
phase was extracted with EtOAc $(5 \mathrm{~mL} \times 3)$ and the combined organic phase was dried over $\mathrm{MgSO}_{4}$ and concentrated. The residue was used in next step without further purification.

Solid state: yellow solid

TLC: $R_{f}=0.35(4: 1$, hexanes/EtOAc)

${ }^{1}$ H NMR (400 MHz, $\left.\mathrm{CDCl}_{3}\right) \delta 7.58(\mathrm{~s}, 1 \mathrm{H}), 6.35(\mathrm{~s}, 1 \mathrm{H}), 5.35(\mathrm{~s}, 2 \mathrm{H}), 5.10(\mathrm{~s}, 2 \mathrm{H}), 3.64(\mathrm{~s}, 3 \mathrm{H}), 3.49$ (s, $3 \mathrm{H}), 2.29(\mathrm{~s}, 3 \mathrm{H})$

\section{2-Amino-6,8-bis(methoxymethoxy)-7-methylnaphthalene-1,4-dione (55)}

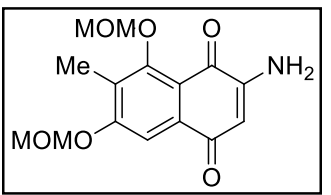
The above crude azide was dissolved in $\mathrm{THF} / \mathrm{H}_{2} \mathrm{O}(5 \mathrm{~mL} / 0.5 \mathrm{~mL})$ and $\mathrm{PPh}_{3}(200 \mathrm{mg})$ was added. After stirring for $1 \mathrm{~h}$ at RT, aqueous workup followed by FC (silica gel; $0 \rightarrow 30 \%$, EtOAc/hexanes) gave pure amine 55 (124 mg, 75\%).

Solid state: yellow solid

TLC: $R_{f}=0.25(4: 1$, hexanes/EtOAc)

${ }^{1}$ H NMR $\left(400 \mathrm{MHz}, \mathrm{CDCl}_{3}\right) \delta 7.62(\mathrm{~s}, 1 \mathrm{H}), 5.92(\mathrm{~s}, 1 \mathrm{H}), 5.35$ (s, 2H), 5.19 (br s, 2H), 5.05 (s, 2H), 3.66 $(\mathrm{s}, 3 \mathrm{H}), 3.52(\mathrm{~s}, 3 \mathrm{H}), 2.30(\mathrm{~s}, 3 \mathrm{H})$

${ }^{13} \mathbf{C}$ NMR $\left(100 \mathrm{MHz}, \mathrm{CDCl}_{3}\right) \delta 183.1,179.6,161.0,158.0,149.5,134.9,126.9,116.7,107.9,103.4$, $101.6,94.4,58.0,56.8,10.1$

MS (ES) calculated for $\mathrm{C}_{15} \mathrm{H}_{18} \mathrm{NO}_{6}[\mathrm{M}+\mathrm{H}]^{+} 308.1$, found 308.1

\section{7-Bromo-5,8-dimethoxy-1,3-bis(methoxymethoxy)-2-methylnaphthalene (57)}

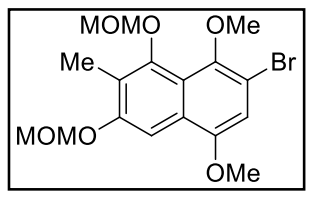

To a solution of bromide $54(370 \mathrm{mg}, 1.0 \mathrm{mmol})$ in ether $(10 \mathrm{~mL})$ was added $\mathrm{Na}_{2} \mathrm{~S}_{2} \mathrm{O}_{4}$ (1.39 g, $8 \mathrm{mmol})$ in $\mathrm{H}_{2} \mathrm{O}(2 \mathrm{~mL})$ at RT. After $30 \mathrm{~min}$, water $(10 \mathrm{~mL})$ was added and the mixture was extracted with ether $(10 \mathrm{~mL})$ three times. The combined organic phase was dried over $\mathrm{MgSO}_{4}$ and concentrated. The residue was dissolved in DMF (10 mL) and $\mathrm{NaH}$ (120 mg, $3.0 \mathrm{mmol})$ was added under an Ar atmosphere. After the mixture was stirred for $15 \mathrm{~min}$ at RT, MeI $(220 \mu \mathrm{L}, 4.0 \mathrm{mmol})$ was added and the mixture was stirred for $12 \mathrm{~h}$ at RT. Water $(10 \mathrm{~mL})$ and EtOAc $(10 \mathrm{~mL})$ were added. The crude was extracted with EtOAc $(10 \mathrm{~mL} \times 3)$ and the combined organic phase was dried over $\mathrm{MgSO}_{4}$ and concentrated. The residue was purified by FC (silica gel; $0 \rightarrow 15 \%$, EtOAc/hexanes) to give pure bromide 57 (312 mg, 78\%).

Physical state: pale yellow oil

TLC: $R_{f}=0.55(5: 1$, hexanes/EtOAc)

${ }^{1}$ H NMR $\left(500 \mathrm{MHz}, \mathrm{CDCl}_{3}\right) \delta 7.57(\mathrm{~s}, 1 \mathrm{H}), 6.86(\mathrm{~s}, 1 \mathrm{H}), 5.35(\mathrm{~s}, 2 \mathrm{H}), 5.06(\mathrm{~s}, 2 \mathrm{H}), 3.94(\mathrm{~s}, 3 \mathrm{H}), 3.81(\mathrm{~s}$, $3 \mathrm{H}), 3.63(\mathrm{~s}, 3 \mathrm{H}), 3.54(\mathrm{~s}, 3 \mathrm{H}), 2.43(\mathrm{~s}, 3 \mathrm{H})$

${ }^{13}$ C NMR (125 MHz, $\left.\mathrm{CDCl}_{3}\right): \delta 154.4,151.6,150.8,145.3,126.7,124.0,119.0,112.3,108.2,101.9$, $100.4,94.6,61.8,58.1,56.6,56.1,10.7$ 
MS (ES) calculated for $\mathrm{C}_{17} \mathrm{H}_{21} \mathrm{BrO}_{6} \mathrm{Na}[\mathrm{M}+\mathrm{Na}]^{+}$423.0, found 423.0

5,8-Dimethoxy-1,3-bis(methoxymethoxy)-2-methylnaphthalene (60)

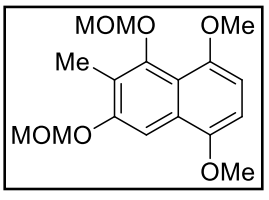

Method A: Trost Procedure [Trost, B. M.; Pearson, W. H. J. Am. Chem. Soc. 1981, $103,2485-2487]$

To a solution of $57(64 \mathrm{mg}, 0.16 \mathrm{mmol})$ in dry THF $(1.0 \mathrm{~mL})$ was added $n \mathrm{BuLi}(70 \mu \mathrm{L}$, 2.5 $\mathrm{M}$ in hexanes, 1.1 eq.) at $-78{ }^{\circ} \mathrm{C}$. After stirring in 30 minutes, magnesium bromide ethyl etherate (41 $\mathrm{mg}, 0.16 \mathrm{mmol}$ ) was added in one portion quickly under an Ar atmosphere. The resulting solution was transferred into the solution of azidomethylphenyl sulfide $(29 \mathrm{mg}, 0.176 \mathrm{mmol})$ in dry THF $(0.3 \mathrm{~mL})$ via a cannula at $-78{ }^{\circ} \mathrm{C}$. After $2 \mathrm{~h}$, the reaction mixture was warmed to $0{ }^{\circ} \mathrm{C}$ and then quenched with saturated aqueous ammonium chloride $(1 \mathrm{~mL})$. The organic phase was dried over $\mathrm{MgSO}_{4}$ and concentrated under vacuum. The resulting crude triazene 59 was dissolved in degassed THF and methanol $(0.15 \mathrm{~mL}$ of each) and $50 \%$ aqueous potassium hydroxide solution $(0.15 \mathrm{~mL})$ was added slowly. After stirring for $6 \mathrm{~h}$, saturated aq. $\mathrm{NaHCO}_{3}(0.5 \mathrm{~mL})$ and EtOAc $(1 \mathrm{~mL})$ were added. The aqueous phase was extracted with EtOAc $(1 \mathrm{~mL} \times 3)$ and the combined organic phases were dried over $\mathrm{MgSO}_{4}$ and concentrated under vacuum. The crude residue was purified by FC (silica gel, $0 \rightarrow 15 \%$, EtOAc/hexanes) to yield compound $60(32 \mathrm{mg}, 62 \%)$ as a yellow oil. Before column purification, TLC indicated the presence of a more polar compound (presumably amine 56). However, this compound was unstable and decomposed during further attempted purification.

\section{Method B: From $\mathrm{NH}_{4} \mathrm{Cl}$ quenching of intermediate 58a}

To a solution of dimethyl ether $57(212 \mathrm{mg}, 0.5 \mathrm{mmol})$ in THF $(2 \mathrm{~mL})$ was added $n$-BuLi $(2.5 \mathrm{M}$ in hexanes, $220 \mu \mathrm{L}$ ) at $-78{ }^{\circ} \mathrm{C}$. After stirring for $30 \mathrm{~min}$ at that temperature, the solution quenched with saturated aqueous ammonium chloride $(10 \mathrm{~mL})$. After extraction with EtOAc, drying over $\mathrm{MgSO}_{4}$, and concentration in vacuo, the residue was purified by FC (silica gel; $0 \rightarrow 10 \%$, EtOAc/hexanes) to give pure compound 60 (116 mg, 72\%).

\section{Method C: From 67}

To a solution of $67(100 \mathrm{mg}, 0.203 \mathrm{mmol})$ in EtOAc $(5 \mathrm{~mL})$ was bubbled in $\mathrm{H}_{2}$ gas over $45 \mathrm{~min}$ in the presence of $\mathrm{Pd} / \mathrm{C}(10 \mathrm{mg}, 10 \mathrm{wt} . \%)$. After filtration over a short pad of Celite ${ }^{\circledR}$, the solvent was removed in vacuo. The crude residue ( $\sim 99 \mathrm{mg}$, without further purification) was dissolved in $\mathrm{CH}_{2} \mathrm{Cl}_{2}(2 \mathrm{~mL})$, and ${ }^{i} \operatorname{Pr}_{2}$ NEt $(141 \mu \mathrm{L}, 0.813 \mathrm{mmol})$, followed by the addition of $\mathrm{MOMCl}(46 \mu \mathrm{L}, 0.61 \mathrm{mmol})$. The resulting reaction mixture was stirred for 4 hours at ambient temperature. Saturated aq. $\mathrm{NaHCO}_{3}(5 \mathrm{~mL})$ and $\mathrm{CH}_{2} \mathrm{Cl}_{2}(5 \mathrm{~mL})$ were added to quench the reaction. The aqueous phase was washed with EtOAc $(5 \mathrm{~mL} \times$ 3). The combined organic phase were dried over $\mathrm{Na}_{2} \mathrm{SO}_{4}$, and concentrated in vacuo. The crude residue 
was purified by flash column (silica gel, $0 \rightarrow 20 \%$, EtOAc/hexanes) to yield compound $\mathbf{6 0}$ (51 $\mathrm{mg}, 78 \%$ yield over 2 steps).

Physical state: yellow oil

TLC: $R_{f}=0.5(4: 1$, hexanes/EtOAc)

${ }^{1}$ H NMR (400 MHz, $\left.\mathrm{CDCl}_{3}\right) \delta 7.74(\mathrm{~d}, J=9.1 \mathrm{~Hz}, 1 \mathrm{H}), 7.37(\mathrm{~s}, 1 \mathrm{H}), 7.13(\mathrm{~d}, J=9.2 \mathrm{~Hz}, 1 \mathrm{H}), 5.34$ (s, 2H), $5.11(\mathrm{~s}, 2 \mathrm{H}), 3.96(\mathrm{~s}, 3 \mathrm{H}), 3.94(\mathrm{~s}, 3 \mathrm{H}), 3.66(\mathrm{~s}, 3 \mathrm{H}), 3.52(\mathrm{~s}, 3 \mathrm{H}), 2.32(\mathrm{~s}, 3 \mathrm{H})$

${ }^{13}$ C NMR $\left(100 \mathrm{MHz}, \mathrm{CDCl}_{3}\right) \delta 154.9,152.1,148.5,142.3,128.9,128.2,120.8,118.4,112.6,100.0,98.5$, $94.5,60.8,57.8,56.7,56.3,10.3$

MS (ES) calculated for $\mathrm{C}_{17} \mathrm{H}_{23} \mathrm{O}_{6}[\mathrm{M}+\mathrm{H}]^{+} 323.2$, found 323.2

\section{5,8-Dimethoxy-1,3-bis(methoxymethoxy)-2-methyl-7-nitronaphthalene (61)}

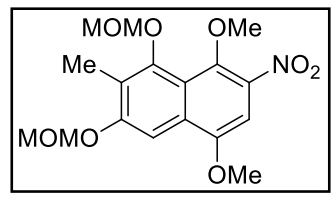

A mixture of $\mathrm{Cu}\left(\mathrm{NO}_{3}\right)_{2} \cdot\left(\mathrm{H}_{2} \mathrm{O}\right)_{2.5}(25 \mathrm{mg}, 0.11 \mathrm{mmol})$ and $\mathrm{CaCl}_{2}(25 \mathrm{mg})$ was dissolved in $\mathrm{Ac}_{2} \mathrm{O}(1.0 \mathrm{~mL})$ and stirred for $10 \mathrm{~min}$ at $\mathrm{RT}$, then cooled to $-40{ }^{\circ} \mathrm{C}$. $\mathrm{A}$ solution of $60(32 \mathrm{mg}, 0.1 \mathrm{mmol})$ in $\mathrm{Ac}_{2} \mathrm{O}(0.2 \mathrm{~mL})$ was added dropwise. After stirring for $1 \mathrm{~h}$ at $-40{ }^{\circ} \mathrm{C}$, ether $(1 \mathrm{~mL})$ and water $(1 \mathrm{~mL})$ were added. The organic phase was concentrated in vacuo, and the crude residue was dried over $\mathrm{MgSO}_{4}$, and purified by FC (silica gel; $5 \% \rightarrow 20 \%$, EtOAc/hexanes) to yield pure nitronaphthalene 61 (26 mg, 71\%).

Physical state: yellow solid

TLC: $R_{f}=0.4$ (hexanes/EtOAc, $\left.4: 1\right)$

${ }^{1}$ H NMR (400 MHz, $\left.\mathrm{CDCl}_{3}\right) \delta 7.61(\mathrm{~s}, 1 \mathrm{H}), 7.07(\mathrm{~s}, 1 \mathrm{H}), 5.37(\mathrm{~s}, 2 \mathrm{H}), 5.03(\mathrm{~s}, 2 \mathrm{H}), 3.99$ (s, 3H), 3.93 (s, $3 \mathrm{H}), 3.61(\mathrm{~s}, 3 \mathrm{H}), 3.53(\mathrm{~s}, 3 \mathrm{H}), 2.41(\mathrm{~s}, 3 \mathrm{H})$

${ }^{13}$ C NMR (150 MHz, $d_{6}$-DMSO) $\delta$ 153.7, 150.8, 150.2, 143.4, 125.3, 123.2, 121.8, 118.2, 105.9, 101.0, 99.9, 94.1, 61.2, 57.3, 56.1, 55.8, 10.3

MS (ES) calculated for $\mathrm{C}_{17} \mathrm{H}_{22} \mathrm{NO}_{8}[\mathrm{M}+\mathrm{H}]^{+} 368.1$, found 368.1

\section{1,4-Dimethoxy-6,8-bis(methoxymethoxy)-7-methylnaphthalen-2-amine (56)}

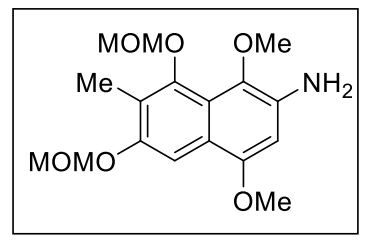

To a solution of $61(9.2 \mathrm{mg}, 25 \mu \mathrm{mol})$ in EtOAc $(1 \mathrm{~mL})$ was added Pd/C (10 wt. \%, $5 \mathrm{mg}$ ) at ambient temperature. The reaction mixture was stirred for 30 minutes under $\mathrm{H}_{2}$ atmosphere (during the process, the color of the solution changed from yellow to colorless). After filtering over a short pad of Celite ${ }^{\circledR}$, the solvent was removed in vacuo, and the residue was dissolved in $\mathrm{CH}_{2} \mathrm{Cl}_{2}(100 \mu \mathrm{L})$ intermediately! (Note: the amine 56 was unstable in air and must be freshly prepared for next step.) 


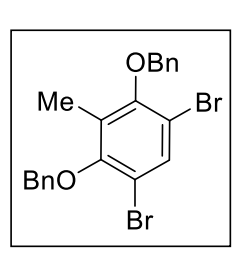

Compound 63 was synthesized according to the literature: Nakata, M.; Wada, S.; Tatsuta, K.; Kinoshita, M. Bull. Chem. Soc. Jpn. 1985, 58, 1801.

To a solution of 2-methylresorcinol (2.48 g, $20 \mathrm{mmol})$ in $\mathrm{CH}_{2} \mathrm{Cl}_{2}(200 \mathrm{~mL})$ was added $\mathrm{Br}_{2}$ (2.01 $\mathrm{mL}, 40 \mathrm{mmol})$ dropwise. After stirring for $2 \mathrm{~h}$, the solvent was removed in vacuo and the crude 4,6-dibromo-2-methylbenzene-1,3-diol (5.54 g, 99\%) was used directly for next step without further purification.

To a solution of 4,6-dibromo-2-methylbenzene-1,3-diol (2.0 g, $7.1 \mathrm{mmol})$ and potassium carbonate (2.26 g, $17.8 \mathrm{mmol})$ in acetone $(20 \mathrm{~mL})$, benzyl bromide $(2.1 \mathrm{~mL}, 17.66 \mathrm{mmol})$ was added dropwise. The resulting mixture was stirred at $60{ }^{\circ} \mathrm{C}$ for $5 \mathrm{~h}$ and filtered with Celite ${ }^{\circledR}$. The solvent was removed and the crude residue was purified by FC (silica gel; $0 \rightarrow 3 \%$ EtOAc/hexanes) to yield 63 (3.04 g, 93\%). All spectral details match those reported in: Nakata, M.; Wada, S.; Tatsuta, K.; Kinoshita, M. Bull. Chem. Soc. Jpn. 1985, 58, 1801-1806.

Physical state: off-white solid

TLC: $R_{f}=0.35(25: 1$, hexanes/EtOAc)

${ }^{1}$ H NMR $\left(600 \mathrm{MHz}, \mathrm{CDCl}_{3}\right) \delta 7.71(\mathrm{~s}, 1 \mathrm{H}), 7.54-7.50(\mathrm{~m}, 4 \mathrm{H})$, 7.44-7.37 (m, 6H), $4.94(\mathrm{~s}, 4 \mathrm{H}), 2.23(\mathrm{~s}$, $3 \mathrm{H})$

${ }^{13}$ C NMR $\left(150 \mathrm{MHz}, \mathrm{CDCl}_{3}\right) \delta 154.4,136.6,133.5,129.7,128.7,128.5,128.4,113.3,74.8,11.5$

MS (ES) calculated for $\mathrm{C}_{21} \mathrm{H}_{19} \mathrm{Br}_{2} \mathrm{O}_{3}[\mathrm{M}+\mathrm{H}]^{+} 461.0$; found 461.0

\section{5,7-Bis(benzyloxy)-8-bromo-6-methyl-1,4-dihydro-1,4-epoxynaphthalene (64)}

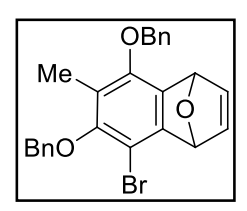

To a solution of ${ }^{i} \operatorname{Pr}_{2} \mathrm{NH}(1.2 \mathrm{~mL}, 8.6 \mathrm{mmol})$ in THF $(15 \mathrm{~mL})$ was added $n$-BuLi $(2.5 \mathrm{M}$ in hexanes, $3.13 \mathrm{~mL}, 7.8 \mathrm{mmol}$ ) at $-78{ }^{\circ} \mathrm{C}$. After stirring for $10 \mathrm{~min}$, the solution was warmed to $0{ }^{\circ} \mathrm{C}$ and stirred for an additional $15 \mathrm{~min}$, then cooled back to $-78{ }^{\circ} \mathrm{C}$. To this solution was added a solution of compound $63(3.0 \mathrm{~g}, 6.5 \mathrm{mmol})$ and furan $(5 \mathrm{~mL})$ in THF $(30 \mathrm{~mL})$ over a $1 \mathrm{~h}$ period. The resulting mixture was allowed to stir overnight. The reaction was quenched by the addition of pH 7 phosphate buffer $(50 \mathrm{~mL})$. The aqueous layer was extracted with ether $(30 \mathrm{~mL} \times 3)$ and the combined organic phase was washed with brine, dried over $\mathrm{MgSO}_{4}$ and concentrated in vacuo. The crude was purified by FC ( silica gel; $0 \rightarrow 10 \%$, EtOAc/hexanes) to give compound 64 (2.6 g, 89\%). All spectral details match those reported in: Nakata, M.; Wada, S.; Tatsuta, K.; Kinoshita, M. Bull. Chem. Soc. Jpn. 1985, 58, 1801-1806.

Physical state: yellow oil

TLC: $R_{f}=0.70$ (4:1 hexanes/EtOAc) 
${ }^{1}$ H NMR $\left(400 \mathrm{MHz}, \mathrm{CDCl}_{3}\right) \delta 7.52(\mathrm{~m}, 2 \mathrm{H}), 7.39(\mathrm{~m}, 8 \mathrm{H}), 7.03(\mathrm{dd}, J=5.6,1.9 \mathrm{~Hz}, 1 \mathrm{H}), 6.73(\mathrm{dd}, J=$ 5.5, $1.8 \mathrm{~Hz}, 1 \mathrm{H}), 5.93$ (br s, 1H), 5.78 (br s, 1H), 5.06 (d, J=12.2 Hz, 1H), 5.01 (d, J=12.2 Hz, 1H), 4.88 $(\mathrm{d}, J=12.2 \mathrm{~Hz}, 1 \mathrm{H}), 4.83(\mathrm{~d}, J=12.2 \mathrm{~Hz}, 1 \mathrm{H}), 2.15(\mathrm{~s}, 3 \mathrm{H})$

${ }^{13}$ C NMR $\left(100 \mathrm{MHz}, \mathrm{CDCl}_{3}\right) \delta 151.8,150.6,149.6,143.2,142.3,137.1,133.8,129.0,128.7,128.5$, $127.8,123.2,105.0,83.4,82.8,75.2,74.7,11.4$

MS (ES) calculated for $\mathrm{C}_{25} \mathrm{H}_{22} \mathrm{BrO}_{3}[\mathrm{M}+\mathrm{H}]^{+} 449.1$, found 449.1

\section{5,7-Bis(benzyloxy)-8-bromo-6-methylnaphthalen-1-ol (65a)}

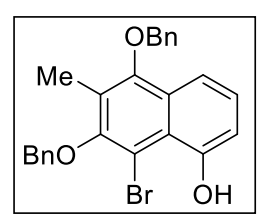

To a solution of benzyne cycloadduct $64(610 \mathrm{mg}, 1.36 \mathrm{mmol})$ and 2,6-di- ${ }^{t}$ butylpridine (1.89 mL, $8.54 \mathrm{mmol})$ in $\mathrm{CH}_{2} \mathrm{Cl}_{2}(14 \mathrm{~mL})$ was added TMSOTf $(1.29 \mathrm{~mL}, 7.12 \mathrm{mmol})$ at $0 \mathrm{C}$. After stirring for $5 \mathrm{~min}$ at this temperature and $3 \mathrm{~h}$ at RT, TBAF (1.0 M in THF, 2.8 $\mathrm{mL}$ ) was added at $0{ }^{\circ} \mathrm{C}$. After stirring for an additional $15 \mathrm{~min}$, the reaction was quenched with saturated aq. $\mathrm{NaHCO}_{3}(15 \mathrm{~mL})$, extracted with EtOAc $(15 \mathrm{~mL})$, dried with $\mathrm{MgSO}_{4}$ and concentrated in vacuum to get the crude product (orange oil). The crude product was purified by FC (silica gel; $0 \rightarrow 15 \%$, EtOAc/hexanes) to yield pure 65a (469 mg, 77\%). The regiochemistry of the ringopening isomerization was established via analysis of the NOESY spectrum of compound $\mathbf{6 5 b}$ (see below).

Physical state: yellow solid

TLC: $R_{f}=0.5(4: 1$, hexanes/EtOAc)

${ }^{1}$ H NMR $\left(400 \mathrm{MHz}, \mathrm{CDCl}_{3}\right) \delta 8.35(\mathrm{~s}, 1 \mathrm{H}), 7.74(\mathrm{dd}, J=8.4,1.2 \mathrm{~Hz}, 1 \mathrm{H}), 7.48-7.55(\mathrm{~m}, 4 \mathrm{H}), 7.30-7.47$ (m, 7H), $7.04(\mathrm{dd}, J=7.7,1.2 \mathrm{~Hz}, 1 \mathrm{H}), 4.95(\mathrm{~s}, 4 \mathrm{H}), 2.37$ (s, 3H)

${ }^{13} \mathrm{C}$ NMR $\left(100 \mathrm{MHz}, \mathrm{CDCl}_{3}\right) \delta 153.9,153.0,152.7,136.8,136.4,128.6,128.4,128.1,128.0,127.9$, $126.7,124.2,120.2,114.7,113.3,105.5,75.5,74.6,30.9,11.2$

MS (ES) calculated for $\mathrm{C}_{25} \mathrm{H}_{22} \mathrm{BrO}_{3}[\mathrm{M}+\mathrm{H}]^{+} 449.1$, found 449.1

Elucidation of the structure of compound 65a via NOESY spectral analysis of derivative 65b
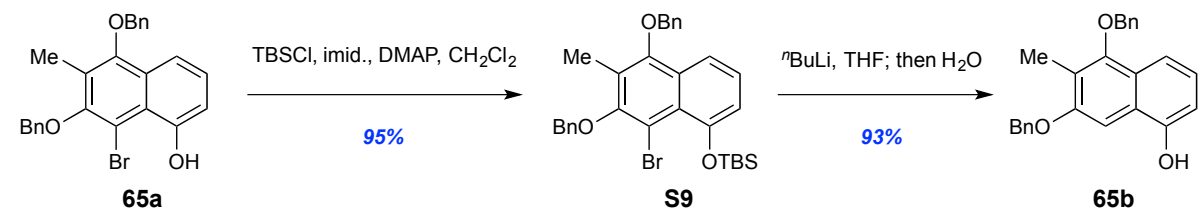

((5,7-Bis(benzyloxy)-8-bromo-6-methylnaphthalen-1-yl)oxy)(tert-butyl)dimethylsilane (S9)

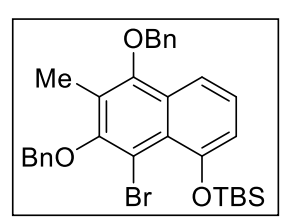

A solution of 65a (44.8 mg, $0.1 \mathrm{mmol})$, imidazole (13.6 mg, $0.2 \mathrm{mmol})$, DMAP (6.1 $\mathrm{mg}, 0.05 \mathrm{mmol})$, and TBSCl $(30.1 \mathrm{mg}, 0.2 \mathrm{mmol})$ in $\mathrm{CH}_{2} \mathrm{Cl}_{2}(0.5 \mathrm{~mL})$ was stirred for 8 $\mathrm{h}$ followed by removal of the solvent in vacuo. The crude residue was purified by FC (silica gel; $0 \rightarrow 5 \%$, EtOAc/hexanes) to yield pure S9 (53.4 mg, 95\%). 
Physical state: yellow solid

TLC: $R_{f}=0.75(5: 1$, hexanes/EtOAc)

${ }^{1}$ H NMR $\left(600 \mathrm{MHz}, \mathrm{CDCl}_{3}\right) \delta$ 7.90-7.80 (m, 1H), $7.66(\mathrm{dt}, J=8.3,1.7 \mathrm{~Hz}, 2 \mathrm{H}), 7.64-7.56(\mathrm{~m}, 2 \mathrm{H}), 7.48-$ $7.41(\mathrm{~m}, 6 \mathrm{H}), 7.38-7.30(\mathrm{~m}, 1 \mathrm{H}), 7.02(\mathrm{dq}, J=7.5,1.3 \mathrm{~Hz}, 1 \mathrm{H}), 5.07$ (d, $J=2.2 \mathrm{~Hz}, 2 \mathrm{H}), 5.02(\mathrm{~d}, J=1.8$ $\mathrm{Hz}, 2 \mathrm{H}), 2.43(\mathrm{~s}, 3 \mathrm{H}), 1.10(\mathrm{~s}, 9 \mathrm{H}), 0.48(\mathrm{~s}, 6 \mathrm{H})$

${ }^{13} \mathbf{C}$ NMR $\left(150 \mathrm{MHz}, \mathrm{CDCl}_{3}\right) \delta 153.8,153.1,152.5,137.2,129.5,128.7,128.6,128.3,128.0,125.7$, $125.0,124.3,115.8,115.4,107.2,75.6,74.3,26.6,19.2,11.3,-3.0$

MS (ES) calculated for $\mathrm{C}_{31} \mathrm{H}_{36} \mathrm{BrO}_{3} \mathrm{Si}[\mathrm{M}+\mathrm{H}]^{+}$563.2, found 563.1

\section{((5,7-Bis(benzyloxy)-6-methylnaphthalen-1-yl)oxy)(tert-butyl)dimethylsilane (65b)}

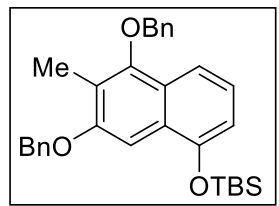

To a solution of $\mathbf{S 9}(40 \mathrm{mg}, 71.1 \mu \mathrm{mol})$ in THF $(0.5 \mathrm{~mL})$, was added ${ }^{n} \mathrm{BuLi}(2.5 \mathrm{M}$ in hexanes, $31.3 \mu \mathrm{L}, 78.3 \mu \mathrm{mol})$ dropwise. After stirring for $10 \mathrm{~min}$, water $(1.0 \mathrm{~mL})$ and EtOAc $(1.0 \mathrm{~mL})$ were added, and the aqueous phase was extracted with EtOAc (1.0 $\mathrm{mL} \times 3$ ). The combined organic phase was dried over $\mathrm{MgSO}_{4}$. After concentration, the crude residue was purified by FC ( silica gel; $0 \rightarrow 5 \%$, EtOAc/hexanes) to yield pure $\mathbf{6 5 b}(53.4 \mathrm{mg}, 93 \%)$.

Physical state: yellow solid

TLC: $R_{f}=0.65(5: 1$, hexanes/EtOAc $)$

${ }^{1}$ H NMR $\left(600 \mathrm{MHz}, \mathrm{CDCl}_{3}\right) \delta 7.69(\mathrm{~d}, J=8.4 \mathrm{~Hz}, 1 \mathrm{H}), 7.63-7.58(\mathrm{~m}, 2 \mathrm{H}), 7.52(\mathrm{dd}, J=7.5,1.6 \mathrm{~Hz}, 2 \mathrm{H})$, 7.49-7.38 (m, 6H), 7.38-7.33 (m, 1H), $7.21(\mathrm{t}, J=8.4 \mathrm{~Hz}, 1 \mathrm{H}), 6.84(\mathrm{dd}, J=7.5,1.0 \mathrm{~Hz}, 1 \mathrm{H}), 5.23(\mathrm{~s}, 2 \mathrm{H})$, $5.02(\mathrm{~s}, 2 \mathrm{H}), 2.41(\mathrm{~s}, 3 \mathrm{H}), 1.12(\mathrm{~s}, 9 \mathrm{H}), 0.29(\mathrm{~s}, 6 \mathrm{H})$

${ }^{13} \mathrm{C}$ NMR $\left(150 \mathrm{MHz}, \mathrm{CDCl}_{3}\right) \delta 155.8,153.3,151.0,137.7,137.3,128.7,128.7,128.2,128.0,127.9$, $127.7,127.3,125.6,123.6,120.6,115.1,112.9,97.9,75.7,70.0,26.1,18.5,10.1,-4.1$

MS (ES) calculated for $\mathrm{C}_{31} \mathrm{H}_{37} \mathrm{BrO}_{3} \mathrm{Si}[\mathrm{M}+\mathrm{H}]^{+} 485.3$, found 485.3

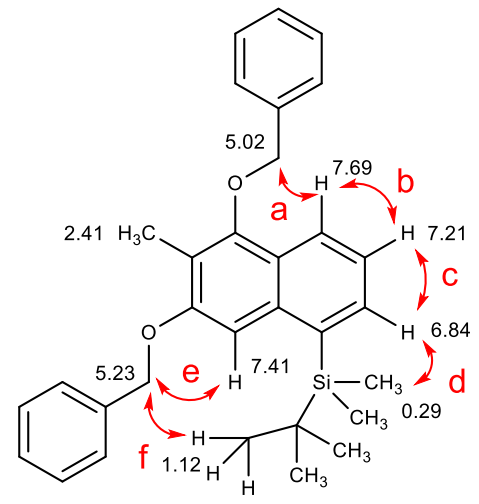

Figure S1. Structure of $65 \mathrm{~b}$ with nOe effects indicated by red arrows 


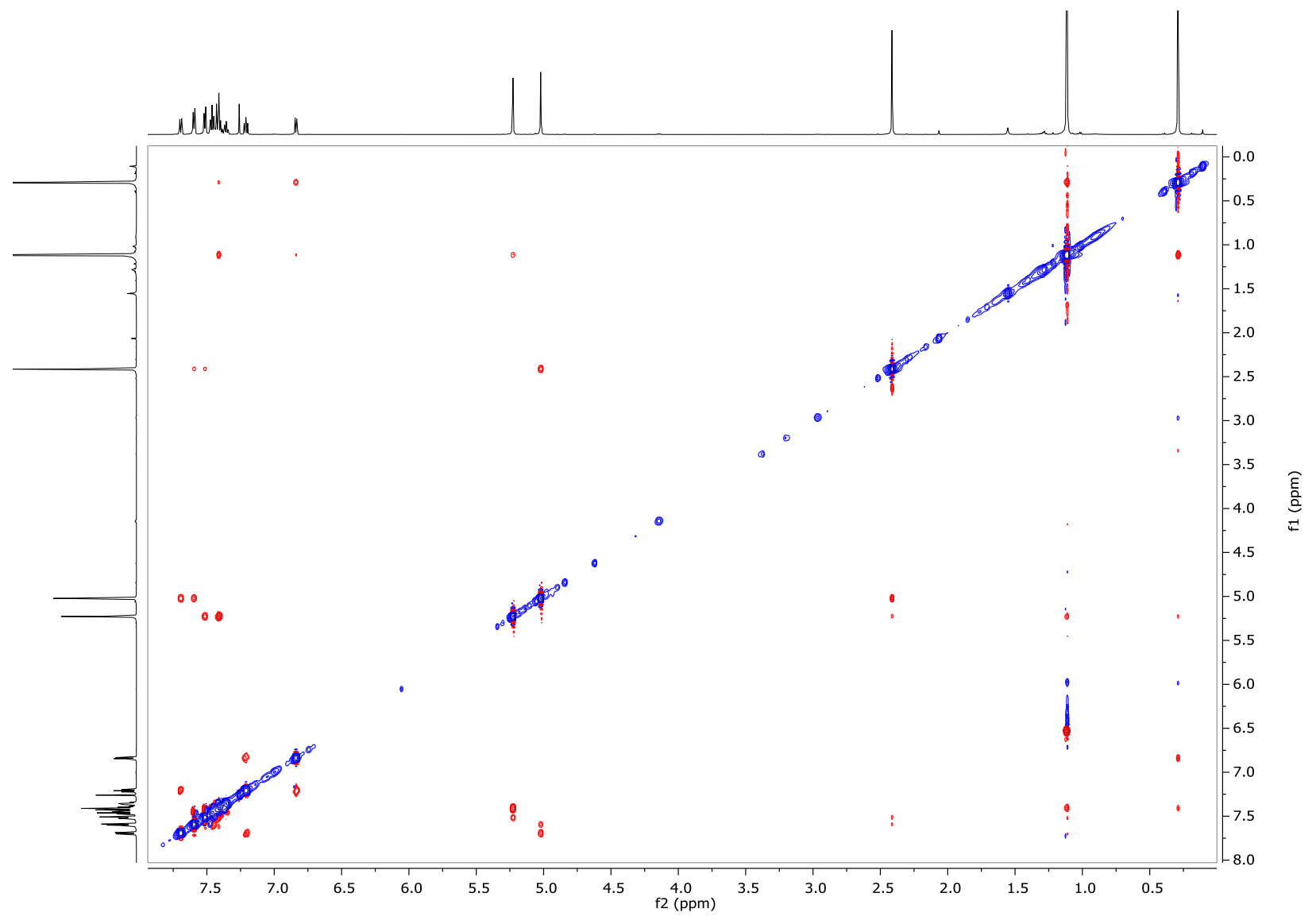

Figure S2. NOESY spectrum of compound $65 \mathrm{~b}\left(600 \mathrm{MHz}, \mathrm{CDCl}_{3}\right)$

\section{5,7-Bis(benzyloxy)-8-bromo-6-methylnaphthalene-1,4-dione (66)}

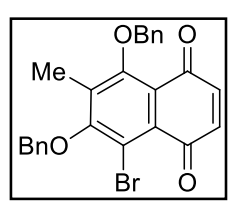

To a solution of compound $65(76 \mathrm{mg}, 0.17 \mathrm{mmol})$ in DMF $(2.0 \mathrm{~mL})$ was added salcomine (Co(salen), $7.6 \mathrm{mg})$. The resulting mixture was stirred under $\mathrm{O}_{2}(1 \mathrm{~atm})$ at $50{ }^{\circ} \mathrm{C}$ overnight.

The reaction was quenched with water and extracted with EtOAc. The combined organic layers were dried over $\mathrm{MgSO}_{4}$ and concentrated in vacuo. The residue was purified by FC (silica gel; $10 \% \rightarrow 50 \%$, EtOAc/hexanes) to give the pure naphthoquinone 66 (58 mg, 73\%).

Physical state: yellow-red solid

TLC: $R_{f}=0.3(1: 1$, EtOAc/hexanes $)$

${ }^{1}$ H NMR $\left(400 \mathrm{MHz}, \mathrm{CDCl}_{3}\right) \delta 7.66(\mathrm{~d}, J=10.5 \mathrm{~Hz}, 1 \mathrm{H}), 7.50(\mathrm{~m}, 1 \mathrm{H}), 7.30-7.44(\mathrm{~m}, 9 \mathrm{H}), 6.30(\mathrm{~d}, J=$ $10.5 \mathrm{~Hz}, 1 \mathrm{H}), 4.98(\mathrm{~s}, 2 \mathrm{H}), 4.87(\mathrm{~s}, 2 \mathrm{H}), 2.24(\mathrm{~s}, 3 \mathrm{H})$

${ }^{13} \mathbf{C}$ NMR $\left(100 \mathrm{MHz}, \mathrm{CDCl}_{3}\right) \delta 180.0,178.3,158.3,155.8,139.4,135.7,135.6,135.2,129.2,129.0$, $128.8,128.4,128.2,127.0,126.1,118.7,76.7,75.0,11.6$

MS (ES) calculated for $\mathrm{C}_{25} \mathrm{H}_{20} \mathrm{BrO}_{4}[\mathrm{M}+\mathrm{H}]^{+} 463.1$, found 463.1 


\section{1,3-Bis(benzyloxy)-4-bromo-5, 8-dimethoxy-2-methylnaphthalene (67)}

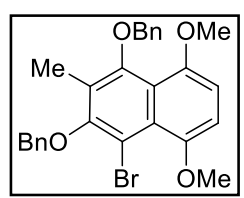

To a solution of naphthoquinone $66(58 \mathrm{mg}, 0.126 \mathrm{mmol})$ in ether $(1.5 \mathrm{~mL})$ and water $(0.5$ $\mathrm{mL})$ was added $\mathrm{Na}_{2} \mathrm{~S}_{2} \mathrm{O}_{4}(175 \mathrm{mg}, 1.0 \mathrm{mmol})$. The resulting mixture was stirred at $\mathrm{RT}$ for

$15 \mathrm{~min}$ and then quenched with water, extracted with ether, dried over $\mathrm{MgSO}_{4}$ and concentrated in vacuum. The crude product was dissolved in DMF $(1 \mathrm{~mL})$, followed by the addition of $\mathrm{NaH}(9.5 \mathrm{mg}, 0.38 \mathrm{mmol})$ and $\mathrm{MeI}(50 \mu \mathrm{L}, 0.91 \mathrm{mmol})$. The resulting mixture was stirred at RT overnight, and quenched with water. After extraction with EtOAc, and drying over $\mathrm{MgSO}_{4}$, the solvent was removed in vacuo to give a residue that was purified by FC (silica gel; $10 \% \rightarrow 40 \%$, EtOAc/hexanes) to give product 67 (50 mg, $80 \%)$.

Physical state: yellow oil

TLC: $R_{f}=0.65(1: 5$, EtOAc/hexanes)

${ }^{1} \mathbf{H}$ NMR $\left(400 \mathrm{MHz}, \mathrm{CDCl}_{3}\right) \delta 7.90(\mathrm{~d}, J=9.3 \mathrm{~Hz}, 1 \mathrm{H}), 7.30-7.62(\mathrm{~m}, 10 \mathrm{H}), 7.26(\mathrm{~d}, J=9.2 \mathrm{~Hz}, 1 \mathrm{H})$, $5.01(\mathrm{~s}, 2 \mathrm{H}), 4.93(\mathrm{~s}, 2 \mathrm{H}), 3.98(\mathrm{~s}, 3 \mathrm{H}), 3.93(\mathrm{~s}, 3 \mathrm{H}), 2.35(\mathrm{~s}, 3 \mathrm{H})$

${ }^{13}$ C NMR $\left(100 \mathrm{MHz}, \mathrm{CDCl}_{3}\right) \delta 156.7,152.1,148.6,142.0,137.2,128.9,128.6,127.8,127.3,120.5$, $118.4,112.1,100.0,96.2,70.0,60.7,57.9,56.6,10.3$

MS (ES) calculated for $\mathrm{C}_{27} \mathrm{H}_{25} \mathrm{BrO}_{4} \mathrm{Na}[\mathrm{M}+\mathrm{Na}]^{+}$515.1, found 515.1

\section{Methyl-6,8-bis(benzyloxy)-7-methyl-1,4-dihydro-1,4-epoxynaphthalene-5-carboxylate (68)}

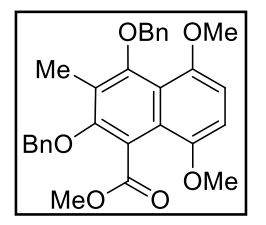

To a solution of $67(910 \mathrm{mg}, 2.02 \mathrm{mmol})$ in THF $(20 \mathrm{~mL})$ was added ${ }^{n} \mathrm{BuLi}(1.65 \mathrm{~mL}$, $2.63 \mathrm{mmol}, 1.6 \mathrm{M}$ in $\mathrm{THF})$ dropwise at $-78^{\circ} \mathrm{C}$. After stirring for $10 \mathrm{~min}$ at $-78{ }^{\circ} \mathrm{C}$, $\mathrm{ClCO}_{2} \mathrm{Me}(313 \mu \mathrm{L}, 4.05 \mathrm{mmol})$ was added. The resulting orange solution was stirred at $-78^{\circ} \mathrm{C}$ for $4 \mathrm{~h}$ and then slowly warmed to RT overnight. The reaction was quenched with water $(20 \mathrm{~mL})$, and the aqueous layer was extracted with EtOAc $(20 \mathrm{~mL} \times 3)$. The combined organic layers were washed with brine, dried with $\mathrm{MgSO}_{4}$ and concentrated in vacuum. The crude residue was purified by FC (silica gel, $0 \rightarrow 20 \%$, EtOAc/hexanes) to yield the product $\mathbf{6 8}(828 \mathrm{mg}, 95 \%)$.

Physical state: yellow oil

TLC: $R_{f}=0.3(1: 4$, EtOAc/hexanes $)$

${ }^{1}$ H NMR $\left(400 \mathrm{MHz}, \mathrm{CDCl}_{3}\right) \delta 7.80-7.72(\mathrm{~m}, 1 \mathrm{H}), 7.50-7.22(\mathrm{~m}, 10 \mathrm{H}), 7.12(\mathrm{dd}, J=9.2,1.5 \mathrm{~Hz}, 1 \mathrm{H})$, $5.21(\mathrm{~s}, 2 \mathrm{H}), 5.12(\mathrm{~s}, 2 \mathrm{H}), 3.95(\mathrm{~s}, 3 \mathrm{H}), 3.90(\mathrm{~s}, 3 \mathrm{H}), 3.66(\mathrm{~s}, 3 \mathrm{H}), 2.36(\mathrm{~s}, 3 \mathrm{H})$

${ }^{13}$ C NMR (100 MHz, $\left.\mathrm{CDCl}_{3}\right)$ 169.7, 154.6, 154.2, 149.4, 142.1, 137.1, 128.7, 128.5, 128.3, 128.1, 125.8, $122.1,121.0,118.7,118.1,114.3,76.7,75.9,61.2,56.6,52.2,10.5$

MS (ES) calculated for $\mathrm{C}_{29} \mathrm{H}_{29} \mathrm{O}_{6}[\mathrm{M}+\mathrm{H}]^{+} 473.2$, found 473.2 
Methyl 2,4-dihydroxy-5,8-dimethoxy-3-methyl-1-naphthoate (69)

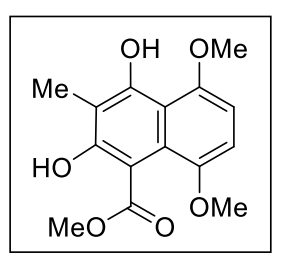

To a solution of $68(9.4 \mathrm{mg}, 20 \mu \mathrm{mol})$ in EtOAc $(1 \mathrm{~mL})$ was added $\mathrm{Pd} / \mathrm{C}(10 \mathrm{wt} . \%, 5$ mg) at ambient temperature. The reaction mixture was stirred for 2 hours under $\mathrm{H}_{2}$ atmosphere. After filtering over a short pad of Celite ${ }^{\circledR}$, the solvent was removed in vacuo. The crude residue was purified by flash column (silica gel, $10 \rightarrow 40 \%$, EtOAc/hexanes) to yield 69 (5.8 $\mathrm{mg}, 99 \%$ yield).

Physical state: off-white solid

TLC: $R_{f}=0.3(2: 1$, EtOAc/hexanes)

${ }^{1}$ H NMR $\left(600 \mathrm{MHz}, \mathrm{CDCl}_{3}\right) 9.31(\mathrm{~s}, 1 \mathrm{H}), 7.84(\mathrm{~d}, J=9.1 \mathrm{~Hz}, 1 \mathrm{H}), 7.13(\mathrm{~d}, J=9.1 \mathrm{~Hz}, 1 \mathrm{H}), 5.50$ (br s, $1 \mathrm{H}), 3.98(\mathrm{~s}, 3 \mathrm{H}), 3.92(\mathrm{~s}, 3 \mathrm{H}), 3.74(\mathrm{~s}, 3 \mathrm{H}), 2.25$ (s, 3H)

${ }^{13}$ C NMR $\left(150 \mathrm{MHz}, \mathrm{CDCl}_{3}\right) \delta 172.6,158.4,153.5,151.2,142.4,126.4,118.2,116.7,111.5,105.5,99.4$, $61.1,56.5,52.6,8.4$

MS (ES) calculated for $\mathrm{C}_{15} \mathrm{H}_{17} \mathrm{O}_{6}[\mathrm{M}+\mathrm{H}]^{+} 293.1$, found 293.1

Methyl 5,8-dimethoxy-2,4-bis(methoxymethoxy)-3-methyl-1-naphthoate (70) (Attempted)

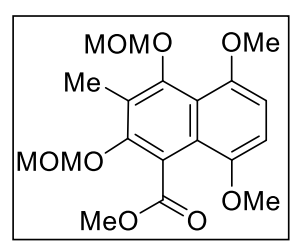

To a solution of $69(7.3 \mathrm{mg}, 25 \mu \mathrm{mol})$ in $\mathrm{CH}_{2} \mathrm{Cl}_{2}$ was added $\mathrm{MOMCl}(7.6 \mu \mathrm{L}, 100$ $\mu \mathrm{mol})$ and ${ }^{i} \operatorname{Pr}_{2} \mathrm{NEt}(13 \mu \mathrm{L}, 75 \mu \mathrm{mol})$ under an Ar atmosphere. The resulting dark green solution was allowed to stir for a couple of hours at RT. TLC indicated complete decomposition during this process.

\section{Methyl 6,8-bis(benzyloxy)-7-methyl-1,4-dihydro-1,4-epoxynaphthalene-5-carboxylate (72)}

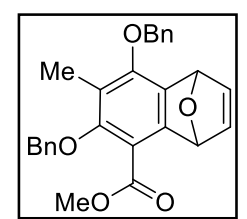

To a solution of $64(1.34 \mathrm{~g}, 3.0 \mathrm{mmol})$ in THF $(30 \mathrm{~mL})$ was added $n$-BuLi $(1.5 \mathrm{~mL}, 2.5 \mathrm{M}$ in hexanes) dropwise over $5 \mathrm{~min}$ at $-78^{\circ} \mathrm{C}$. After stirring for $15 \mathrm{~min}$ at $-78{ }^{\circ} \mathrm{C}$, methyl chloroformate $(696 \mu \mathrm{L}, 9.0 \mathrm{mmol})$ was added in one portion. After stirring at $-78{ }^{\circ} \mathrm{C}$ for an additional $2 \mathrm{~h}$, the mixture was warmed to RT and water $(20 \mathrm{~mL})$ and ethyl acetate $(20 \mathrm{~mL})$ were added. The aqueous was extracted with ethyl acetate $(3 \times 20 \mathrm{~mL})$. The combined organic phases were dried over $\mathrm{MgSO}_{4}$ and concentrated in vacuo. The crude residue was purified by FC (silica gel; $5 \% \rightarrow 20 \%$ EtOAc/hexanes) to yield ester $72(1.22 \mathrm{~g}, 95 \%)$.

Physical state: yellow oil

TLC: $R_{f}=0.31(1: 4$, EtOAc/hexanes $)$

${ }^{1}$ H NMR $\left(400 \mathrm{MHz}, \mathrm{CDCl}_{3}\right) \delta$ 7.51-7.27 (m, 10H), $7.05(\mathrm{dd}, J=5.5,1.9 \mathrm{~Hz}, 1 \mathrm{H}), 6.74(\mathrm{dd}, J=5.5,1.8$ Hz, 1H), 6.09 (br s, 1H), 5.95 (br s, 1H), 5.21 (d, $J=11.8 \mathrm{~Hz}, 1 \mathrm{H}), 5.09$ (d, $J=11.8 \mathrm{~Hz}, 1 \mathrm{H}), 4.95$ (d, $J=$ $10.6 \mathrm{~Hz}, 1 \mathrm{H}), 4.78(\mathrm{~d}, J=10.6 \mathrm{~Hz}, 1 \mathrm{H}), 3.86(\mathrm{~s}, 3 \mathrm{H}), 2.14(\mathrm{~s}, 3 \mathrm{H})$

${ }^{13}$ C NMR $\left(100 \mathrm{MHz}, \mathrm{CDCl}_{3}\right) \delta 169.7,154.6,154.2,149.4,142.1,137.1,128.6,128.5,128.3,128.1$, $127.9,125.8,122.1,120.9,118.7,118.0,114.3,76.7,75.9,61.2,56.6,52.2,10.3$ 
MS (ES) calculated for $\mathrm{C}_{27} \mathrm{H}_{25} \mathrm{O}_{5}[\mathrm{M}+\mathrm{H}]^{+} 429.2$, found 429.2

\section{Methyl 8-hydroxy-2,4-bis(methoxymethoxy)-3-methyl-1-naphthoate (75)}

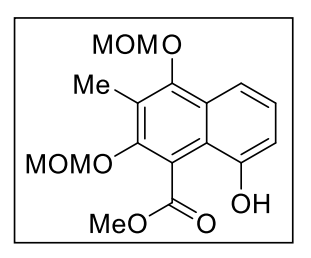

To a solution of $72(610 \mathrm{mg}, 1.42 \mathrm{mmol})$ and 2,6-di-butylpridine $(1.89 \mathrm{~mL}, 8.54$ mmol) in $\mathrm{CH}_{2} \mathrm{Cl}_{2}(14 \mathrm{~mL})$ was added TMSOTf $(1.29 \mathrm{~mL}, 7.12 \mathrm{mmol})$ at $0{ }^{\circ} \mathrm{C}$. The mixture was stirred for $5 \mathrm{~min}$ at $0{ }^{\circ} \mathrm{C}$ and $3 \mathrm{~h}$ at $\mathrm{RT}$, followed by quenching with saturated aq. $\mathrm{NaHCO}_{3}(20 \mathrm{~mL})$, extraction with EtOAc $(3 \times 20 \mathrm{~mL})$, drying over $\mathrm{MgSO}_{4}$ and concentration in vacuo. The crude material (orange oil) thus obtained was used immediately in the next step. The crude residue was dissolved in EtOAc $(14 \mathrm{~mL})$ followed by the addition of $\mathrm{Pd} / \mathrm{C}(50$ $\mathrm{mg}, 10 \mathrm{wt} \%)$. The reaction mixture was stirred under $\mathrm{H}_{2}(1 \mathrm{~atm})$ for $2 \mathrm{~h}$ at $\mathrm{RT}$ and filtered through a pale of Celite ${ }^{\circledR}$. After removal of the solvent, the crude product was dissolved in $\mathrm{CH}_{2} \mathrm{Cl}_{2}(14 \mathrm{~mL})$, followed by the addition of ${ }^{i} \operatorname{Pr}_{2} \mathrm{NEt}(735 \mu \mathrm{L}, 4.3 \mathrm{mmol}, 3.0$ equiv) and $\mathrm{MOMCl}$ (216 $\mu \mathrm{L}, 2.84 \mathrm{mmol}, 2.0$ equiv) at $0{ }^{\circ} \mathrm{C}$. The resulting solution was stirred at RT overnight, quenched with water $(10 \mathrm{~mL})$, extracted with EtOAc $(3 \times 20 \mathrm{~mL})$, dried with $\mathrm{MgSO}_{4}$ and concentrated in vacuo. The crude was purified by FC (silica gel; $0 \rightarrow 50 \%$ EtOAc/hexanes) to yield bis-MOM ether 75 (172 mg, 36\% for 3 steps) as a dark red oil. A less polar fraction contained primarily the tris-MOM ether 74 (97 mg, 18\%). The latter could be partially deprotected to yield bis-MOM ether 75 as follows: To a solution of 74 (97 mg, $0.256 \mathrm{mmol})$ in $\mathrm{CH}_{2} \mathrm{Cl}_{2}(3$ $\mathrm{mL})$ was added $\mathrm{BCl}_{3}\left(280 \mu \mathrm{L}, 1.0 \mathrm{M}\right.$ solution in $\left.\mathrm{CH}_{2} \mathrm{Cl}_{2}\right)$ at $-78^{\circ} \mathrm{C}$. The resulting solution was stirred for $1 \mathrm{~h}$ and quenched with sat. $\mathrm{NaHCO}_{3}$ solution $(3 \mathrm{~mL})$. The aqueous phase was extracted with EtOAc $(3 \times 3$ $\mathrm{mL}$ ) and the combined organic phase were dried over $\mathrm{MgSO}_{4}$, and concentrated in vacuo. The crude residue was purified by flash column (silica gel, $0 \rightarrow 50 \%$ gradient of EtOAc/hexanes) to yield bis-MOM ether 75 (68 mg, 79\%). The regiochemistry of the ring-opening isomerization was established via analysis of the NOESY spectrum of compound 74 (see below).

\section{Characterization data for 74 (tris-MOM ether):}

Physical state: yellow oil

TLC: $R_{f}=0.3(1: 1$, EtOAc/hexanes)

${ }^{1}$ H NMR $\left(600 \mathrm{MHz}, \mathrm{CDCl}_{3}\right) \delta 7.72(\mathrm{dd}, J=8.4,1.0 \mathrm{~Hz}, 1 \mathrm{H}), 7.35(\mathrm{dd}, J=8.5,7.7 \mathrm{~Hz}, 1 \mathrm{H}), 7.09(\mathrm{dd}, J=$ 7.7, $0.9 \mathrm{~Hz}, 1 \mathrm{H}), 5.24(\mathrm{~s}, 2 \mathrm{H}), 5.12(\mathrm{~s}, 2 \mathrm{H}), 5.09(\mathrm{~s}, 2 \mathrm{H}), 3.96(\mathrm{~s}, 3 \mathrm{H}), 3.66(\mathrm{~d}, J=0.4 \mathrm{~Hz}, 3 \mathrm{H}), 3.62(\mathrm{~s}$, $3 \mathrm{H}), 3.51(\mathrm{~d}, J=0.5 \mathrm{~Hz}, 3 \mathrm{H}), 2.45(\mathrm{~s}, 3 \mathrm{H})$

${ }^{13}$ C NMR $\left(150 \mathrm{MHz}, \mathrm{CDCl}_{3}\right) \delta 169.8,153.5,152.2,152.0,127.8,126.0,124.0,121.9,119.2,116.1$, $109.6,101.2,100.3,95.3,58.1,57.8,56.6,52.3,11.5$

MS (ES) calculated for $\mathrm{C}_{19} \mathrm{H}_{27} \mathrm{O}_{8}[\mathrm{M}+\mathrm{H}]^{+} 381.2$, found 381.2

\section{Characterization data for 75 (bis-MOM ether):}

Physical state: yellow oil 
TLC: $R_{f}=0.2(1: 1$, EtOAc/hexanes $)$

${ }^{1}$ H NMR $\left(600 \mathrm{MHz}, \mathrm{CDCl}_{3}\right) \delta 7.61(\mathrm{dd}, J=8.5,0.9 \mathrm{~Hz}, 1 \mathrm{H}), 7.21(\mathrm{dd}, J=8.5,7.5 \mathrm{~Hz}, 1 \mathrm{H}), 6.79(\mathrm{dd}, J=$ 7.5, $1.0 \mathrm{~Hz}, 1 \mathrm{H}), 5.12(\mathrm{~s}, 2 \mathrm{H}), 5.10(\mathrm{~s}, 2 \mathrm{H}), 3.92(\mathrm{~s}, 3 \mathrm{H}), 3.66(\mathrm{~s}, 3 \mathrm{H}), 3.62(\mathrm{~s}, 3 \mathrm{H}), 2.44(\mathrm{~s}, 3 \mathrm{H})$

${ }^{13}$ C NMR $\left(150 \mathrm{MHz}, \mathrm{CDCl}_{3}\right) \delta 170.9,153.5,152.0,150.0,128.0,126.0,123.9,120.7,119.0,115.0$, $111.2,101.1,100.2,58.1,57.8,52.9,11.5$

MS (ES) calculated for $\mathrm{C}_{17} \mathrm{H}_{21} \mathrm{O}_{7}[\mathrm{M}+\mathrm{H}]^{+}$337.1, found 337.1

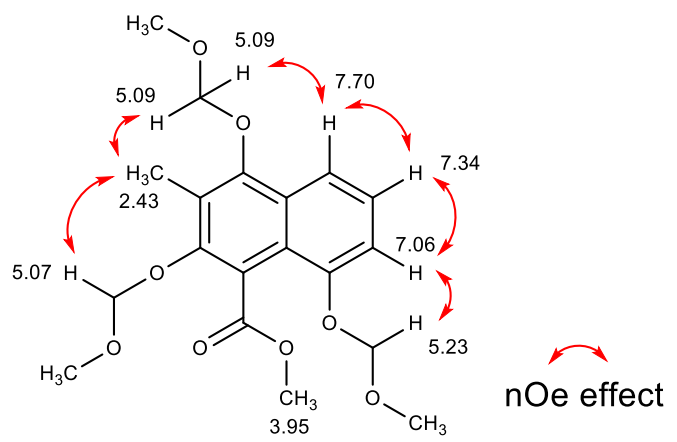

Figure S3. Structure of 74 with nOe effects indicated by red arrows

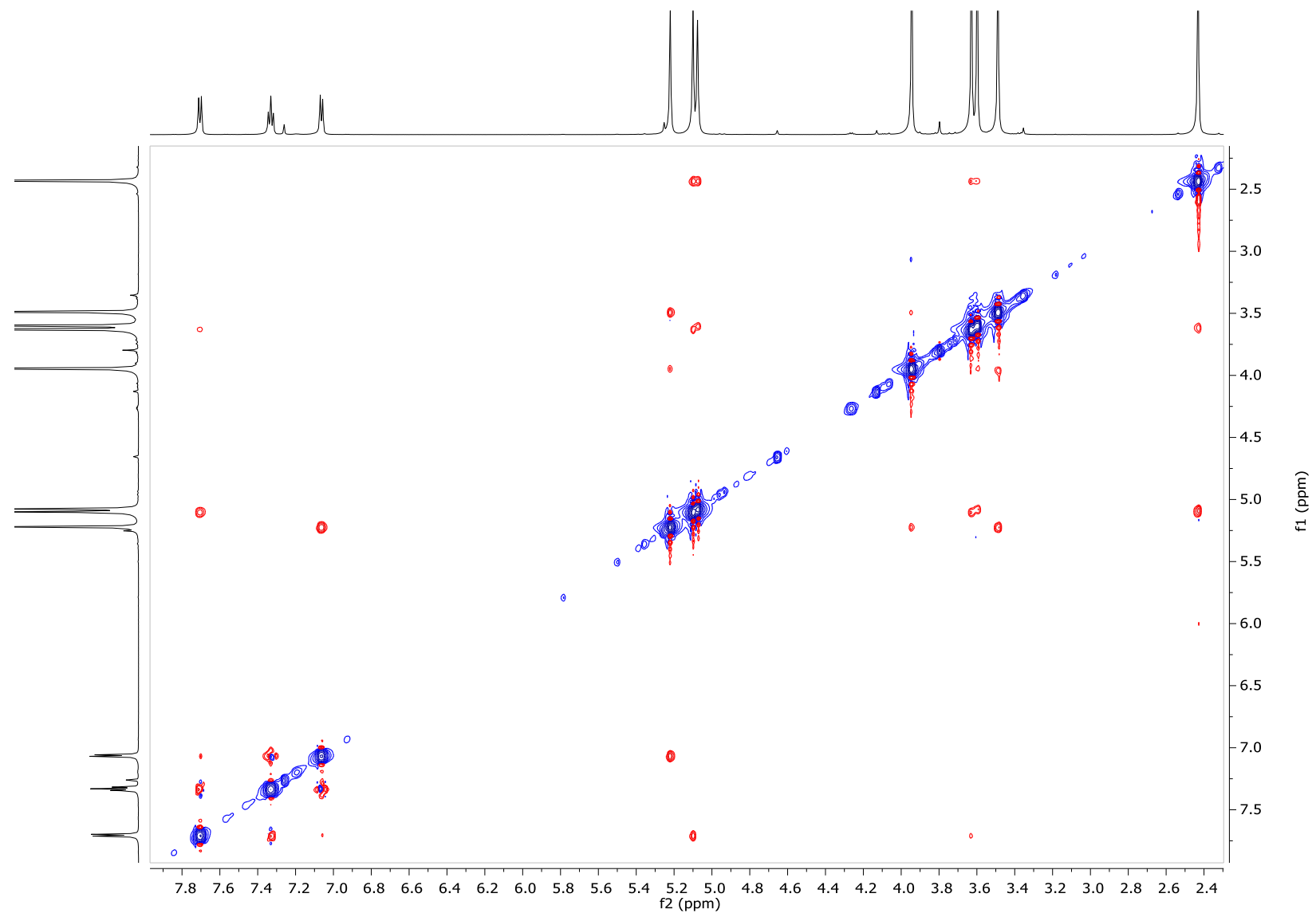

Figure S4. NOESY spectrum of compound $74\left(600 \mathrm{MHz} \mathrm{CDCl}_{3}\right)$ 


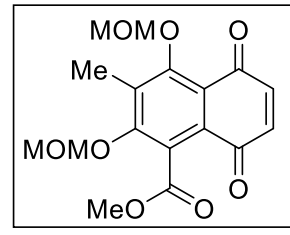

To a solution of $75(76 \mathrm{mg}, 0.226 \mathrm{mmol})$ in DMF $(2.5 \mathrm{~mL})$ was added salcomine catalyst (Co(salen) 71, $7.6 \mathrm{mg}, 0.1$ equiv). The resulting mixture was stirred under $\mathrm{O}_{2}$ $(1 \mathrm{~atm})$ at $50{ }^{\circ} \mathrm{C}$ overnight. The reaction was quenched with water $(10 \mathrm{~mL})$, extracted

with EtOAc $(10 \mathrm{~mL})$, dried with $\mathrm{MgSO}_{4}$ and concentrated in vacuo. The crude residue was purified by preparative TLC (1:1, EtOAc/hexanes) to yield pure naphthoquinone 76 (54 mg, 0.154 mmol, 68\%).

Physical state: red solid

TLC: $R_{f}=0.3$ (1:1, EtOAc/hexanes)

${ }^{1}$ H NMR $\left(600 \mathrm{MHz}, \mathrm{CDCl}_{3}\right): 7.82(\mathrm{~d}, J=10.5 \mathrm{~Hz}, 1 \mathrm{H}), 6.40(\mathrm{~d}, J=10.4 \mathrm{~Hz}, 1 \mathrm{H}), 5.06(\mathrm{~s}, 2 \mathrm{H}), 5.05(\mathrm{~s}$, 2H), $3.98(\mathrm{~s}, 3 \mathrm{H}), 3.64(\mathrm{~s}, 3 \mathrm{H}), 3.58(\mathrm{~s}, 3 \mathrm{H}), 2.35$ (s, 3H)

${ }^{13}$ C NMR (150 MHz, $\left.\mathrm{CDCl}_{3}\right) \delta 180.1,177.5,167.0,156.4,140.1,136.1,128.9,127.7,126.6,125.2$, $101.2,101.0,58.3,58.0,53.4,12.1$

MS (ES) calculated for $\mathrm{C}_{17} \mathrm{H}_{18} \mathrm{O}_{8} \mathrm{Na}[\mathrm{M}+\mathrm{Na}]^{+} 373.1$; found 373.1

Methyl 5,8-dimethoxy-2,4-bis(methoxymethoxy)-3-methyl-1-naphthoate (70)

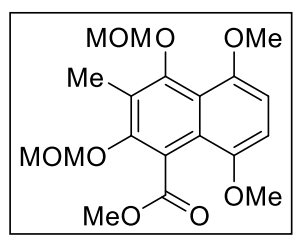

To a solution of $76(57 \mathrm{mg}, 0.163 \mathrm{mmol})$ in ether $(1 \mathrm{~mL})$ and water $(0.2 \mathrm{~mL})$ was added $\mathrm{Na}_{2} \mathrm{~S}_{2} \mathrm{O}_{4}$ (284 mg, $1.63 \mathrm{mmol}$ ). The resulting mixture was stirred at RT for 15 min and then quenched with water, extracted with ether, dried with $\mathrm{MgSO}_{4}$ and concentrated in vacuo. The crude product was dissolved in DMF $(1 \mathrm{~mL})$, followed by the addition of $\mathrm{NaH}(60 \%$ in mineral, $20 \mathrm{mg}, 3.0$ equiv, $0.489 \mathrm{mmol})$ and $\mathrm{MeI}(41 \mu \mathrm{L}, 4.0$ equiv, 0.652 $\mathrm{mmol})$. The resulting mixture was stirred at RT overnight, quenched with water, extracted with EtOAc $(10 \mathrm{~mL})$, dried with $\mathrm{MgSO}_{4}$ and concentrated in vacuo. The crude residue was purified by preparative TLC (2:3, EtOAc/hexanes) to yield the pure compound 70 (42 mg, 68\%).

Physical state: yellow oil

TLC: $R_{f}=0.4$ (2:3, EtOAc/hexanes)

${ }^{1}$ H NMR $\left(400 \mathrm{MHz}, \mathrm{CDCl}_{3}\right) \delta 7.84(\mathrm{~d}, J=9.4 \mathrm{~Hz}, 1 \mathrm{H}), 7.25(\mathrm{~d}, J=9.4 \mathrm{~Hz}, 1 \mathrm{H}), 5.11(\mathrm{~s}, 2 \mathrm{H}), 5.10(\mathrm{~s}$, 2H), 3.97 (s, 3H), 3.94 (s, 3H), 3.84 (s, 3H), 3.66 (s, 3H), 3.62 (s, 3H), 2.40 (s, 3H)

${ }^{13}$ C NMR $\left(100 \mathrm{MHz}, \mathrm{CDCl}_{3}\right) \delta 169.5,153.4,152.6,149.3,141.8,125.6,122.4,121.1,118.8,118.1$, $114.3,100.9,100.1,61.2,57.9,57.6,56.4,52.2,11.1$

MS (ES) calculated for $\mathrm{C}_{19} \mathrm{H}_{25} \mathrm{O}_{8}[\mathrm{M}+\mathrm{H}]^{+} 381.2$, found 381.2 
Methyl 5,8-dimethoxy-2,4-bis(methoxymethoxy)-3-methyl-6-nitro-1-naphthoate (77)

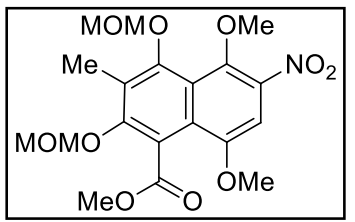

To a solution of 77 (42 $\mathrm{mg}, 0.1 \mathrm{mmol})$ in acetic anhydride $(1.0 \mathrm{~mL})$, $\mathrm{Cu}\left(\mathrm{NO}_{3}\right)_{2} \cdot\left(\mathrm{H}_{2} \mathrm{O}\right)_{2.5}(23.4 \mathrm{mg}, 105 \mu \mathrm{mol}, 1.05$ equiv $)$ and dry $\mathrm{CaCl}_{2}(15 \mathrm{mg}, 135$ $\mu$ mol, 1.35 equiv) were added in one portion. The mixture was stirred at RT for 15 min and cooled down to $-40{ }^{\circ} \mathrm{C}$. Then a solution of $70(38 \mathrm{mg}, 0.1 \mathrm{mmol})$ in acetic anhydride $(0.2 \mathrm{~mL})$ was added dropwise. After stirring at $-40{ }^{\circ} \mathrm{C}$ for $1 \mathrm{~h}$, ether $(2.0 \mathrm{~mL})$ and water $(2.0 \mathrm{~mL})$ were added to the mixture, which was allowed to warm to RT. The aqueous phase was extracted with EtOAc $(3 \times 2 \mathrm{~mL})$. The combined organic phase was dried over $\mathrm{MgSO}_{4}$ and concentrated in vacuo. The crude residue was purified by $\mathrm{FC}(5 \% \rightarrow 25 \%$, EtOAc/hexanes) to afford nitronaphthalene 77 (29.5 mg, 69\%).

Physical state: orange solid

TLC: $R_{f}=0.15(1: 2$, hexanes/EtOAc)

${ }^{1}$ H NMR $\left(500 \mathrm{MHz}, \mathrm{CD}_{3} \mathrm{COCD}_{3}\right) \delta 8.48(\mathrm{~s}, 1 \mathrm{H}), 5.39(\mathrm{~s}, 2 \mathrm{H}), 5.14(\mathrm{~s}, 2 \mathrm{H}), 4.05(\mathrm{~s}, 3 \mathrm{H}), 3.98(\mathrm{~s}, 3 \mathrm{H})$, $3.91(\mathrm{~s}, 3 \mathrm{H}), 3.64(\mathrm{~s}, 3 \mathrm{H}), 3.51(\mathrm{~s}, 3 \mathrm{H}), 2.32(\mathrm{~s}, 3 \mathrm{H})$

${ }^{13}$ C NMR $\left(125 \mathrm{MHz}, \mathrm{CD}_{3} \mathrm{COCD}_{3}\right) \delta 170.7,155.2,152.7,148.9,142.4,129.2,121.0,118.5,118.2,113.1$, $100.2,98.5,94.6,60.1,58.5,57.2,56.3,55.6,9.9$

MS (ES) calculated for $\mathrm{C}_{19} \mathrm{H}_{24} \mathrm{NO}_{10}[\mathrm{M}+\mathrm{H}]^{+} 426.1$, found 426.1

Methyl 6-amino-5,8-dimethoxy-2,4-bis(methoxymethoxy)-3-methyl-1-naphthoate (78)

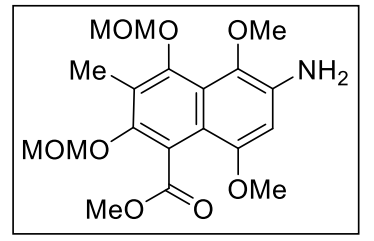

To a solution of $77(10.6 \mathrm{mg}, 25 \mu \mathrm{mol})$ in EtOAc $(1 \mathrm{~mL})$ was added $\mathrm{Pd} / \mathrm{C}(10$ wt. $\%, 5 \mathrm{mg}$ ) at ambient temperature. The reaction mixture was stirred for 45 minutes under $\mathrm{H}_{2}$ atmosphere. After filtering with a short pad of Celite ${ }^{\circledR}$, the solvent was removed in vacuo, and the residue was dissolved in $\mathrm{CH}_{2} \mathrm{Cl}_{2}(100 \mu \mathrm{L})$ intermediately! (Note: the amine $\mathbf{7 8}$ was unstable in air and must be freshly prepared for next step)

$(S, 2 Z, 4 E)-1 H-B e n z o[d][1,2,3]$ triazol-1-yl 6-((4S,5R,6R)-6-((R)-1-((1S,3R,4R,5S)-1,4-dimethyl-2,8-dioxabicyclo[3.2.1]octan-3-yl)ethyl)-2,2,5-trimethyl-1,3-dioxan-4-yl)-2-methylhepta-2,4-dienoate (81)

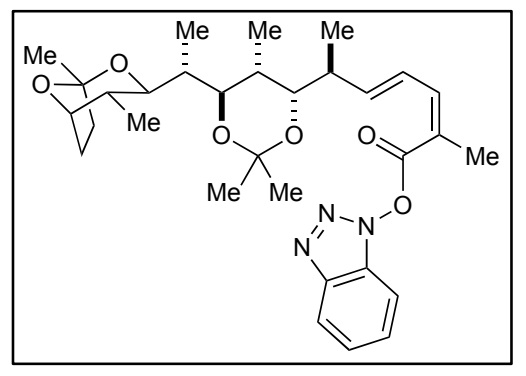

To a solution of dienoic acid S6 (aka 79; $1.0 \mathrm{mg}, 2.3 \mu \mathrm{mol}$ ) and amine $\mathbf{5 5}$ (2.5 mg, $8.1 \mu \mathrm{mol})$ in $\mathrm{CH}_{2} \mathrm{Cl}_{2}(150 \mu \mathrm{L})$ was added HOBt $(1.0 \mathrm{mg}, 6.5$ $\mu \mathrm{mol})$, EDCI (1.0 mg, $6.4 \mu \mathrm{mol})$, and triethylamine $(1 \mu \mathrm{L}, 7.5 \mu \mathrm{mol})$. The mixture was stirred for $2 \mathrm{~h}$ at ambient temperature and concentrated under vacuum. The residue was purified by preparative TLC (hexanes/EtOAc, 4:1) to provide the title compound $\mathbf{8 1}(1.1 \mathrm{mg}, 86 \%)$ as a white solid and recovered amine 55 (2.0 mg, 80\%).

Physical state: white solid 
TLC: $R_{f}=0.25$ (4:1, hexanes/EtOAc)

${ }^{1}$ H NMR $\left(500 \mathrm{MHz}, \mathrm{CDCl}_{3}\right) \delta 7.68(\mathrm{dd}, J=12.0,7.5 \mathrm{~Hz}, 1 \mathrm{H}), 7.56(\mathrm{t}, J=7.6 \mathrm{~Hz}, 1 \mathrm{H}), 7.54-7.49(\mathrm{~m}, 1 \mathrm{H})$, 7.44-7.40 (m, 1H), $7.22(\mathrm{dd}, J=15.3,11.3 \mathrm{~Hz}, 1 \mathrm{H}), 6.87(\mathrm{~d}, J=10.2 \mathrm{~Hz}, 1 \mathrm{H}), 6.28(\mathrm{dd}, J=10.8,6.4 \mathrm{~Hz}$, $1 \mathrm{H}), 5.36(\mathrm{~m}, 1 \mathrm{H}), 5.13(\mathrm{~d}, J=5.9 \mathrm{~Hz}, 1 \mathrm{H}), 4.21(\mathrm{q}, J=3.8,3.1 \mathrm{~Hz}, 1 \mathrm{H}), 4.15(\mathrm{dd}, J=6.4,3.6 \mathrm{~Hz}, 1 \mathrm{H})$, $3.72(\mathrm{~d}, J=10.8 \mathrm{~Hz}, 1 \mathrm{H}), 3.51$ (dt, $J=10.4,3.4 \mathrm{~Hz}, 1 \mathrm{H}), 3.29$ (dd, $J=9.0,6.4 \mathrm{~Hz}, 1 \mathrm{H}), 2.44(\mathrm{~m}, 1 \mathrm{H})$, 2.29 (d, $J=6.0 \mathrm{~Hz}, 1 \mathrm{H}), 2.25$ (s, 3H), 2.23 (t, $J=7.7 \mathrm{~Hz}, 1 \mathrm{H}), 2.04$ (s, 3H), 2.00-1.75 (m, 5H), 1.43 (s, $3 \mathrm{H}), 0.97$ (d, $J=9.4 \mathrm{~Hz}, 3 \mathrm{H}), 0.87$ (dd, $J=13.3,6.5 \mathrm{~Hz}, 6 \mathrm{H}), 0.68$ (d, $J=6.9 \mathrm{~Hz}, 3 \mathrm{H})$

MS (ES) calculated for $\mathrm{C}_{31} \mathrm{H}_{44} \mathrm{~N}_{3} \mathrm{O}_{6}[\mathrm{M}+\mathrm{H}]^{+}$554.3, found: 554.2

(S,2Z,4E)-N-(1,4-Dimethoxy-6,8-bis(methoxy-methoxy)-7-methyl-naphthalen-2-yl)-6-((4S,5R,6R)-6$((R)-1-((1 S, 3 R, 4 R, 5 S)$-1,4-dimethyl-2,8-dioxabicyclo[3.2.1]octan-3-yl)ethyl)-2,2,5-trimethyl-1,3dioxan-4-yl)-2-methylhepta-2,4-dienamide (84)

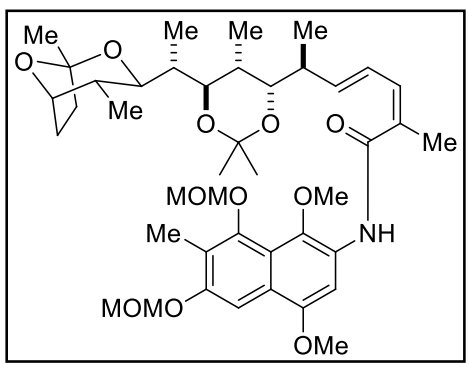

Method A: To a solution of dienoic acid S6 (aka 79; $5.4 \mathrm{mg}, 12.4 \mu \mathrm{mol}$ ) in $\mathrm{CH}_{2} \mathrm{Cl}_{2}(100 \mu \mathrm{L})$ was added HOBt $(3.34 \mathrm{mg}, 24.7 \mu \mathrm{mol})$, EDCI $(3.8 \mathrm{mg}$, $24.7 \mu \mathrm{mol})$, and triethyl amine $(3.5 \mu \mathrm{L}, 24.7 \mu \mathrm{mol})$. After stirring for $20 \mathrm{~min}$ at RT, the crude amine $\mathbf{5 6}$ (unstable; prepared immediately before use as described above; $8.5 \mathrm{mg}, 24.7 \mu \mathrm{mol})$ in $\mathrm{CH}_{2} \mathrm{Cl}_{2}(100 \mu \mathrm{L})$ was added. The yellow solution was stirred for $6 \mathrm{~h}$ under Ar atmosphere. After removing the solvent in vacuo, the crude residue was purified by preparative TLC (4:1, hexanes/EtOAc) to provide pure compound 84 (6.3 mg, 67\%).

Method B: A mixture of $\mathrm{CuI}(0.25 \mathrm{mg}, 10 \mathrm{~mol} \%)$, the crude amide prepared from S6 (aka 80; see intermediate en route to saliniketal A, before acetonide deprotection; $5.8 \mathrm{mg}, 13.3 \mu \mathrm{mol})$, bromide 57 (6.4 $\mathrm{mg}, 15.6 \mu \mathrm{mol}), \mathrm{K}_{3} \mathrm{PO}_{4}(5.6 \mathrm{mg}, 26.6 \mu \mathrm{mol})$, and $N^{\prime}, N^{\prime}$-dimethylethylenediamine $(0.5 \mu \mathrm{L})$ in toluene $(150$ $\mu \mathrm{L}$ ) was stirred at $100{ }^{\circ} \mathrm{C}$ overnight. After removing the solvent in vacuo, the crude residue was purified by preparative TLC (4:1, hexanes/EtOAc) to yield pure compound 84 (5.7 mg, 57\%).

Physical state: pale yellow foam

TLC: $R_{f}=0.45$ (3:2, hexanes/EtOAc)

Optical rotation: $[\alpha]_{D}^{20}=+8.9(c=0.05$ in $\mathrm{MeOH})$

${ }^{1}$ H NMR $\left(600 \mathrm{MHz}, \mathrm{CD}_{3} \mathrm{OD}\right) \delta 7.67$ (s, 1H), 7.05 (br t, $\left.J=5.6 \mathrm{~Hz}, 1 \mathrm{H}\right), 6.86(\mathrm{~s}, 1 \mathrm{H}), 6.37$ (br d, $J=2.4$ $\mathrm{Hz}, 1 \mathrm{H}), 5.84-5.81(\mathrm{~m}, 1 \mathrm{H}), 5.37$ (s, 2H), $5.03(\mathrm{~s}, 2 \mathrm{H}), 4.23-4.20(\mathrm{~m}, 1 \mathrm{H}), 3.98(\mathrm{~s}, 3 \mathrm{H}), 3.80$ (d, J=6.0 Hz, 1H), 3.77 (s, 3H), 3.68 (d, $J=7.8 \mathrm{~Hz}, 1 \mathrm{H}), 3.64$ (s, 3H), 3.52-3.50 (m, 1H), 3.49 (s, 3H), 3.32 (s, 3H), 2.39 (s, 3H), 2.37-2.34 (m, 1H), 1.95 (s, 3H), 1.92-1.78 (m, 7H), 1.75-1.66 (m, 1H), 1.31 (d, $J=27.7 \mathrm{~Hz}$, $3 \mathrm{H}), 1.26(\mathrm{~s}, 3 \mathrm{H}), 1.08-0.79(\mathrm{~m}, 9 \mathrm{H}), 0.68(\mathrm{~d}, J=6.9 \mathrm{~Hz}, 3 \mathrm{H})$ 
${ }^{13}$ C NMR (150 MHz, CD 3 OD) $\delta 173.8,154.0,151.1,150.2,143.7,140.9,132.7,129.7,126.1,123.5$, $121.7,117.9,109.7,105.1,104.8,101.3,99.8,99.2,93.8,80.0,74.0,72.6,72.3,60.1,56.4,54.9,38.4$, $35.4,35.1,33.4,24.1,23.3,22.4,21.9,19.0,14.8,10.8,10.2,9.0,7.8$

MS (ES) calculated for $\mathrm{C}_{42} \mathrm{H}_{62} \mathrm{NO}_{11}[\mathrm{M}+\mathrm{H}]^{+} 756.4$, found: 756.4

$(2 Z, 4 E, 6 S, 7 S, 8 R, 9 R, 10 R)-N-(6,8-D i h y d r o x y-7-m e t h y l-1,4-d i o x 0-1,4-d i h y d r o n a p h t h a l e n-2-y l)-10-$ ((1S,3S,4R,5S)-1,4-dimethyl-2,8-dioxa-bicyclo[3.2.1]octan-3-yl)-7,9-dihydroxy-2,6,8-trimethylundeca-2,4-dienamide (Salinisporamycin, 2)

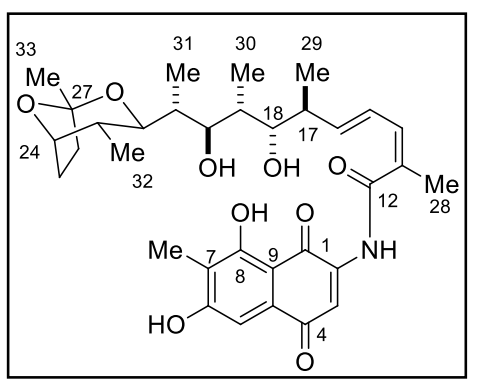

A solution of ceric ammonium nitrate $(5.5 \mathrm{mg}, 10 \mu \mathrm{mol})$ in $1: 1 \mathrm{CH}_{3} \mathrm{CN} / \mathrm{H}_{2} \mathrm{O}$ $(100 \mu \mathrm{L})$ was added to a $0{ }^{\circ} \mathrm{C}$ solution of $84(2.5 \mathrm{mg}, 10.0 \mu \mathrm{mol})$ in $\mathrm{CH}_{3} \mathrm{CN}$ $(200 \mu \mathrm{L})$ and $\mathrm{H}_{2} \mathrm{O}(10 \mu \mathrm{L})$. The resulting solution was stirred at $0{ }^{\circ} \mathrm{C}$ for 15 min and then was poured into $\mathrm{H}_{2} \mathrm{O}(1 \mathrm{~mL})$. The aqueous phase was extracted with $\mathrm{CH}_{2} \mathrm{Cl}_{2}(3 \times 1 \mathrm{~mL})$. The combined organic extracts were dried over $\mathrm{MgSO}_{4}$, filtered, and concentrated in vacuo to give a bright yellow oil. The crude residue (82) was filtered over a short path of silica gel. The residue obtained after removal of the solvent was dissolved in THF/MeOH/H$/ \mathrm{H}_{2} \mathrm{O}(100 \mu \mathrm{L} / 25 \mu \mathrm{L} / 10 \mu \mathrm{L})$ and $\mathrm{NaI}(1.0 \mathrm{mg}, 6.7 \mu \mathrm{mol})$ and $\mathrm{HCl}$ (3N aq., $10 \mu \mathrm{L})$ were added. The resulting mixture was stirred for $24 \mathrm{~h}$ at RT. Saturated aq. $\mathrm{NaHCO}_{3}(150$ $\mu \mathrm{L})$ was added slowly to the reaction mixture followed by EtOAc $(500 \mu \mathrm{L})$. The aqueous phase was extracted with EtOAc $(3 \times 0.5 \mathrm{~mL})$. The combined organic phase was dried over $\mathrm{MgSO}_{4}$, and concentrated in vacuo. The crude residue was purified by preparative TLC $\left(5: 1, \mathrm{CH}_{2} \mathrm{Cl}_{2} / \mathrm{MeOH}\right)$ to give salinisporamycin $(2,1.2 \mathrm{mg}, 62 \%, 2$ steps $)$.

Physical state: off-white foam

TLC: $R_{f}=0.80\left(4: 1, \mathrm{CHCl}_{3} / \mathrm{MeOH}\right)$

Optical rotation: $[\alpha]_{D}^{20}=+10.5\left(c=0.05\right.$ in $\left.\mathrm{CD}_{3} \mathrm{OD}\right)$

${ }^{1}$ H NMR (600 MHz, CD 3 OD) $\delta 7.57(\mathrm{~s}, 1 \mathrm{H}), 6.95$ (s, 1H), $6.79(\mathrm{dd}, J=15.0,11.1 \mathrm{~Hz}, 1 \mathrm{H}), 6.47$ (br d, $J$ $=11.4 \mathrm{~Hz}, 1 \mathrm{H}), 6.03(\mathrm{dd}, J=15.1,7.9 \mathrm{~Hz}, 1 \mathrm{H}), 4.20(\mathrm{dd}, J=6.7,3.4 \mathrm{~Hz}, 1 \mathrm{H}), 3.93(\mathrm{dd}, J=10.6,1.7 \mathrm{~Hz}$, 1H), $3.78(\mathrm{dd}, J=9.1,1.8 \mathrm{~Hz}, 1 \mathrm{H}), 3.51(\mathrm{dd}, J=8.4,4.3 \mathrm{~Hz}, 1 \mathrm{H}), 2.43(\mathrm{ddq}, J=9.2,7.6,6.8 \mathrm{~Hz}, 1 \mathrm{H})$, $2.10(\mathrm{~d}, J=1.4 \mathrm{~Hz}, 3 \mathrm{H}), 2.05(\mathrm{~s}, 3 \mathrm{H}), 1.75-2.06(\mathrm{~m}, 7 \mathrm{H}), 1.39(\mathrm{~s}, 3 \mathrm{H}), 1.01$ (d, $J=7.0 \mathrm{~Hz}, 3 \mathrm{H}), 0.99$ (d, $J$ $=6.8 \mathrm{~Hz}, 3 \mathrm{H}), 0.89(\mathrm{~d}, J=6.9 \mathrm{~Hz}, 3 \mathrm{H}), 0.71(\mathrm{~d}, J=6.8 \mathrm{~Hz}, 3 \mathrm{H})$

${ }^{13}$ C NMR (125 MHz, CD 3 OD) $\delta 187.8,181.3,171.9,170.1,164.5,146.0,143.2,138.6,132.6,129.8$, 127.6, 118.0, 116.4, 112.7, 106.7, 106.5, 81.8, 78.3, 75.8, 75.2, 42.4, 37.1, 36.4, 35.4, 35.4, 24.4, 24.1, $20.7,17.1,13.0,11.3,10.5,8.2$

MS (ES) calculated for $\mathrm{C}_{33} \mathrm{H}_{44} \mathrm{NO}_{9}[\mathrm{M}+\mathrm{H}]^{+} 598.3$, found 598.3 
Table S5. Comparison of ${ }^{1} \mathrm{H}$ and ${ }^{13} \mathrm{C}$ NMR for natural (500 and $\left.125 \mathrm{MHz}, \mathrm{CD}_{3} \mathrm{OD}\right)$ and synthetic salinisporamycin (2, 600 and $\left.125 \mathrm{MHz}, \mathrm{CD}_{3} \mathrm{OD}\right)$

\begin{tabular}{|c|c|c|c|c|}
\hline \multirow[t]{2}{*}{ No. } & \multirow{2}{*}{$\begin{array}{r}\text { Natural } \\
\delta \mathrm{H}, \text { mult. }(J \text { in } \mathrm{Hz})\end{array}$} & \multicolumn{3}{|c|}{ Synthetic } \\
\hline & & $\delta \mathrm{C}$ & $\delta \mathrm{H}$, mult. $(J$ in $\mathrm{Hz})$ & $\delta \mathrm{C}$ \\
\hline 1 & & 181.3 & & 181.3 \\
\hline 2 & & 143.2 & & 143.2 \\
\hline 3 & $7.55, \mathrm{~s}$ & 116.4 & $7.57, \mathrm{~s}$ & 116.4 \\
\hline 4 & & 187.9 & & 187.8 \\
\hline 5 & $6.96, \mathrm{~s}$ & 112.7 & $6.95, \mathrm{~s}$ & 112.7 \\
\hline 6 & & 164.5 & & 164.5 \\
\hline 7 & & 117.8 & & 118.0 \\
\hline 8 & & 172.0 & & 171.9 \\
\hline 9 & & 106.6 & & 106.7 \\
\hline 10 & & 132.5 & & 132.6 \\
\hline 11 & $2.06, \mathrm{~s}$ & 8.2 & $2.05, \mathrm{~s}$ & 8.2 \\
\hline 12 & & 170.1 & & 170.1 \\
\hline 13 & & 129.7 & & 129.8 \\
\hline 14 & 6.46, br d (11.3) & 138.6 & $6.47, \mathrm{dd}(11.4,1.6)$ & 138.6 \\
\hline 15 & $6.79, \mathrm{dd},(15.0,10.9)$ & 127.6 & $6.79, \mathrm{dd}(15.0,11.1)$ & 127.6 \\
\hline 16 & $6.03, \mathrm{dd},(15.0,8.3)$ & 146.0 & $6.03, \mathrm{dd}(15.1,7.9)$ & 146.0 \\
\hline 17 & $2.43, \mathrm{~m},(8.3,7.5)$ & 42.4 & $2.43, \operatorname{ddq}(9.2,7.6,6.8)$ & 42.4 \\
\hline 18 & $3.78, \mathrm{dd},(9.8,1.9)$ & 75.8 & $3.78, \mathrm{dd}(9.1,1.8)$ & 75.8 \\
\hline 19 & $1.83, \mathrm{~m},(6.8,4.5,1.9)$ & 36.4 & $1.75-2.06, \mathrm{~m}(1 \mathrm{H}$ of $7 \mathrm{H})$ & 36.4 \\
\hline 20 & $3.50, \mathrm{dd},(8.3,4.5)$ & 78.4 & $3.51, \mathrm{dd}(8.4,4.3)$ & 78.3 \\
\hline 21 & 1.82, br dq $(8.3,6.8,1.5)$ & 37.1 & $1.75-2.06, \mathrm{~m}(1 \mathrm{H}$ of $7 \mathrm{H})$ & 37.1 \\
\hline 22 & 3.94, br d $(10.5,1.5)$ & 75.2 & $3.93, \mathrm{dd}(10.6,1.7)$ & 75.2 \\
\hline 23 & 1.97, br dq $(10.5,6.8,3.8)$ & 35.4 & $1.75-2.06, \mathrm{~m}(1 \mathrm{H}$ of $7 \mathrm{H})$ & 35.4 \\
\hline 24 & 4.20, br d $(6.8,3.8)$ & 81.8 & $4.20, \mathrm{dd}(6.7,3.4)$ & 81.8 \\
\hline $25 \mathrm{a}$ & $1.93, \mathrm{~m}$ & 24.1 & $1.75-2.06, \mathrm{~m}(1 \mathrm{H}$ of $7 \mathrm{H})$ & 24.1 \\
\hline $25 b$ & $1.88, \mathrm{~m}$ & & $1.75-2.06, \mathrm{~m}(1 \mathrm{H}$ of $7 \mathrm{H})$ & \\
\hline $26 \mathrm{a}$ & $1.80, \mathrm{~m}$ & 35.4 & $1.75-2.06, \mathrm{~m}(1 \mathrm{H}$ of $7 \mathrm{H})$ & 35.4 \\
\hline $26 b$ & $2.01-2.05, \mathrm{~m}$ & & $1.75-2.06, \mathrm{~m}(1 \mathrm{H}$ of $7 \mathrm{H})$ & \\
\hline 27 & & 106.6 & & 106.6 \\
\hline 28 & $2.07, \mathrm{~d}(1.1)$ & 20.7 & $2.10, \mathrm{~d}(1.4)$ & 20.7 \\
\hline 29 & $0.99, \mathrm{~d}(6.8)$ & 17.1 & $0.99, \mathrm{~d}(6.8)$ & 17.1 \\
\hline 30 & $1.00, \mathrm{~d}(6.8)$ & 11.3 & $1.01, \mathrm{~d}(7.0)$ & 11.3 \\
\hline 31 & $0.88, \mathrm{~d}(6.8)$ & 10.5 & $0.89, \mathrm{~d}(6.9)$ & 10.5 \\
\hline 32 & $0.71, \mathrm{~d}(6.8)$ & 13.0 & $0.71, \mathrm{~d}(6.8)$ & 13.0 \\
\hline 33 & $1.39, \mathrm{~s}$ & 24.4 & $1.39, \mathrm{~s}$ & 24.4 \\
\hline
\end{tabular}


Methyl 6-((S,2Z,4E)-6-((4S,5R,6R)-6-((R)-1-((1S,3R,4R,5S)-1,4-Dimethyl-2,8-dioxabicyclo[3.2.1]octan-3-yl)ethyl)-2,2,5-trimethyl-1,3-dioxan-4-yl)-2-methylhepta-2,4-dienamido)-5,8-dimethoxy-2,4bis(methoxymethoxy)-3-methyl-1-naphthoate (85)

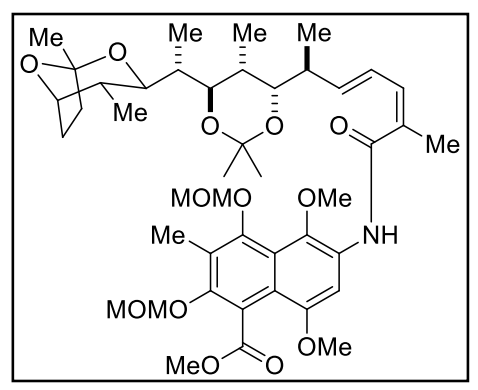

To a solution of dienoic acid S6 (aka 79; $5.4 \mathrm{mg}, 12.4 \mu \mathrm{mol})$ in $\mathrm{CH}_{2} \mathrm{Cl}_{2}(100$ $\mu \mathrm{L})$ was added HOBt (3.34 mg, $24.7 \mu \mathrm{mol})$, EDCI (3.8 mg, $24.7 \mu \mathrm{mol})$, and triethyl amine $(3.5 \mu \mathrm{L}, 24.7 \mu \mathrm{mol})$. After stirring for $20 \mathrm{~min}$ at RT, a solution of the crude amine $\mathbf{7 8}$ (unstable; freshly prepared as described above before use; $0.25 \mathrm{M}$ in $\left.\mathrm{CH}_{2} \mathrm{Cl}_{2}\right)$ in $\mathrm{CH}_{2} \mathrm{Cl}_{2}(100 \mu \mathrm{L})$ was added. The yellow solution was stirred for $6 \mathrm{~h}$ under Ar atmosphere. After removing the solvent in vacuo, the crude residue was purified by preparative TLC (4:1, hexanes/EtOAc) to yield pure compound $\mathbf{8 5}(6.3 \mathrm{mg}, 67 \%)$.

Physical state: pale yellow foam

TLC: $R_{f}=0.30(3: 1$, hexanes/EtOAc)

Optical rotation: $[\alpha]_{D}^{20}=+13.2(c=0.07$ in EtOAc $)$

${ }^{1}$ H NMR (500 MHz, CD 3 OD) $\delta 7.43(\mathrm{~s}, 1 \mathrm{H}), 6.59(\mathrm{dd}, J=15.0,11.1 \mathrm{~Hz}, 1 \mathrm{H}), 6.20(\mathrm{dd}, J=11.4,1.6 \mathrm{~Hz}$, 1H), $5.82(\mathrm{dd}, J=15.1,7.9 \mathrm{~Hz}, 1 \mathrm{H}), 5.38(\mathrm{~s}, 2 \mathrm{H}), 5.14(\mathrm{~s}, 2 \mathrm{H}), 4.22(\mathrm{~m}, 1 \mathrm{H}), 3.99(\mathrm{~s}, 3 \mathrm{H}), 3.99(\mathrm{~s}, 3 \mathrm{H})$, $3.95(\mathrm{~s}, 3 \mathrm{H}), 3.75-3.83(\mathrm{~m}, 2 \mathrm{H}), 3.66(\mathrm{~s}, 3 \mathrm{H}), 3.55(\mathrm{~s}, 3 \mathrm{H}), 3.50-3.56(\mathrm{~m}, 1 \mathrm{H}), 2.30-2.39(\mathrm{~m}, 1 \mathrm{H}), 2.35(\mathrm{~s}$, $3 \mathrm{H}), 2.00(\mathrm{~s}, 3 \mathrm{H}), 1.80-2.00(\mathrm{~m}, 7 \mathrm{H}), 1.43(\mathrm{~s}, 3 \mathrm{H}), 1.29(\mathrm{~s}, 3 \mathrm{H}), 1.26(\mathrm{~s}, 3 \mathrm{H}), 0.81-1.00(\mathrm{~m}[3 \times \mathrm{d}], 9 \mathrm{H})$, $0.74(\mathrm{~d}, J=6.9 \mathrm{~Hz}, 3 \mathrm{H})$

${ }^{13}$ C NMR (125 MHz, CD 3 OD) $\delta 175.2,172.4,156.4,153.7,152.1,150.0,143.3,142.3,134.3,130.2$, $129.7,128.2,122.1,119.7,119.4,113.7,106.4,101.4,99.4,95.6,81.6,78.0,75.7,74.9,61.2,58.9,58.1$, $57.1,56.4,55.3,42.3,37.1,35.7,35.2,35.1,24.9,24.3,24.2,20.9,17.0,14.5,12.8,11.1,10.5$

MS (ES) calculated for $\mathrm{C}_{44} \mathrm{H}_{64} \mathrm{NO}_{13}[\mathrm{M}+\mathrm{H}]^{+} 814.4$; found 814.4

6-((2Z,4E,6S,7S,8R,9R,10R)-10-((1S,3S,4R,5S)-1,4-Dimethyl-2,8-dioxa-bicyclo[3.2.1]octan-3-yl)-7,9dihydroxy-2,6,8-trimethylundeca-2,4-dienamido)-2,4-dihydroxy-3-methyl-5,8-dioxo-5,8-dihydronaphthalene-1-carboxylic acid (Rifsaliniketal, 9)

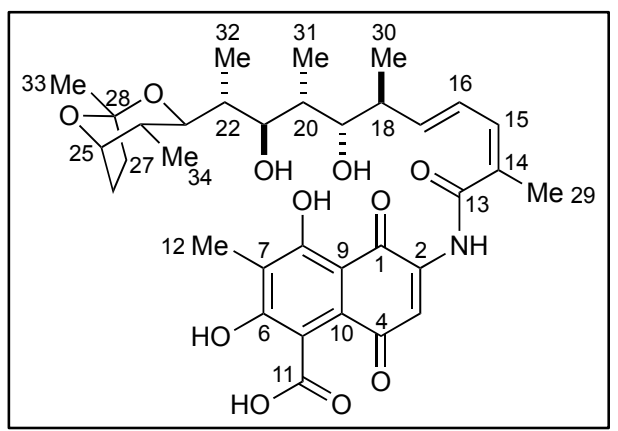

A solution of ceric ammonium nitrate $(5.2 \mathrm{mg}, 10 \mu \mathrm{mol})$ in $1: 1$ $\mathrm{CH}_{3} \mathrm{CN} / \mathrm{H}_{2} \mathrm{O}(100 \mu \mathrm{L})$ was added to a $0{ }^{\circ} \mathrm{C}$ solution of $85(2.3 \mathrm{mg}$, $3.2 \mu \mathrm{mol})$ in $\mathrm{CH}_{3} \mathrm{CN}(100 \mu \mathrm{L})$ and $\mathrm{H}_{2} \mathrm{O}(5 \mu \mathrm{L})$. The resulting solution was stirred at $0{ }^{\circ} \mathrm{C}$ for $15 \mathrm{~min}$ and then was poured into $\mathrm{H}_{2} \mathrm{O}(0.5 \mathrm{~mL})$. The aq. phase was extracted with $\mathrm{CH}_{2} \mathrm{Cl}_{2}(3 \times 1 \mathrm{~mL})$ and the combined organic extracts were dried $\left(\mathrm{MgSO}_{4}\right)$, filtered, 
and concentrated in vacuo. The residue (83) was filtered over a short path of silica gel. The residue obtained after removal of the solvent was dissolved in THF/MeOH/ $\mathrm{H}_{2} \mathrm{O}(50 \mu \mathrm{L} / 15 \mu \mathrm{L} / 10 \mu \mathrm{L})$ and $\mathrm{NaI}$ $(1.0 \mathrm{mg}, 6.7 \mu \mathrm{mol})$ and aq. $\mathrm{HCl}(3 \mathrm{~N}, 10 \mu \mathrm{L})$ were added. The resulting mixture was stirred for $24 \mathrm{~h}$ at $\mathrm{RT}$ after which the solvent was removed and the residue dissolved in $\mathrm{MeOH}(70 \mu \mathrm{L})$. An aq. $\mathrm{LiOH}$ solution $(1.0 \mathrm{M}, 20 \mu \mathrm{L})$ was added and the mixture was stirred at $0{ }^{\circ} \mathrm{C}$ for overnight. Saturated aq. $\mathrm{NaHCO}_{3}(150$ $\mu \mathrm{L})$ was added, followed by extraction with EtOAc $(3 \times 0.5 \mathrm{~mL})$. The combined organic phase was dried $\left(\mathrm{MgSO}_{4}\right)$, and concentrated in vacuo. The residue was purified by preparative TLC $\left(5: 1, \mathrm{CH}_{2} \mathrm{Cl}_{2} / \mathrm{MeOH}\right)$ to give rifsaliniketal $(9,1.0 \mathrm{mg}, 47 \%, 3$ steps $)$.

Physical state: yellow foam

TLC: $R_{f}=0.20$ (EtOAc)

Optical rotation: $[\alpha]_{D}^{20}=+10.0(c=0.05$ in acetone $)$

${ }^{1}$ H NMR $\left(600 \mathrm{MHz}, \mathrm{CD}_{3} \mathrm{OD}\right) \delta 7.66(\mathrm{~s}, 1 \mathrm{H}), 6.78(\mathrm{dd}, J=15.1,11.1 \mathrm{~Hz}, 1 \mathrm{H}), 6.46(\mathrm{dd}, J=11.4,1.6 \mathrm{~Hz}$, 1H), $6.02(\mathrm{dd}, J=15.1,7.9 \mathrm{~Hz}, 1 \mathrm{H}), 4.22(\mathrm{dd}, J=6.7,3.4 \mathrm{~Hz}, 1 \mathrm{H}), 3.95(\mathrm{dd}, J=10.6,1.7 \mathrm{~Hz}, 1 \mathrm{H}), 3.78$ $(\mathrm{dd}, J=9.1,1.8 \mathrm{~Hz}, 1 \mathrm{H}), 3.51(\mathrm{dd}, J=8.4,2.9 \mathrm{~Hz}, 1 \mathrm{H}), 2.43(\mathrm{ddq}, J=9.2,7.6,6.8 \mathrm{~Hz}, 1 \mathrm{H}), 2.16(\mathrm{~s}, 3 \mathrm{H})$, $2.08(\mathrm{~s}, 3 \mathrm{H}), 1.75-2.05(\mathrm{~m}, 7 \mathrm{H}), 1.39$ (s, 3H), 1.01 (d, $J=7.0 \mathrm{~Hz}, 3 \mathrm{H}), 0.99$ (d, J=6.8 Hz, 3H), 0.89 (d, $J$ $=6.9 \mathrm{~Hz}, 3 \mathrm{H}), 0.72(\mathrm{~d}, J=6.9 \mathrm{~Hz}, 3 \mathrm{H})$

${ }^{13}$ C NMR (125 MHz, CD 3 OD) $\delta$ 186.4, 184.0, 175.8, 169.9, 166.8, 162.8, 145.6, 141.3, 138.2, 129.5, $128.8,127.5,120.9,117.6$ (2), 106.9, 106.4, 81.5, 78.2, 75.6, 75.0, 42.3, 36.9, 36.0 35.2, 35.1, 24.8, 24.1, $20.5,16.9,12.8,11.1,10.3,8.0$

MS (ES) calculated for $\mathrm{C}_{34} \mathrm{H}_{44} \mathrm{NO}_{11}[\mathrm{M}+\mathrm{H}]^{+}$642.3; found 642.3 
Table S6. Comparison of ${ }^{1} \mathrm{H}$ and ${ }^{13} \mathrm{C}$ NMR for natural (600 and $125 \mathrm{MHz}, \mathrm{CD}_{3} \mathrm{OD}$ ) and synthetic rifsaliniketal $\left(9,600\right.$ and $\left.125 \mathrm{MHz}, \mathrm{CD}_{3} \mathrm{OD}\right)$

\begin{tabular}{|c|c|c|c|c|}
\hline \multirow[t]{2}{*}{ No. } & \multirow{2}{*}{$\begin{array}{r}\text { Natural } \\
\delta \mathrm{H}, \text { mult. }(J \text { in } \mathrm{Hz}) \\
\end{array}$} & \multicolumn{3}{|c|}{ Synthetic } \\
\hline & & $\delta \mathrm{C}$ & $\delta \mathrm{H}$, mult. $(J$ in $\mathrm{Hz})$ & $\delta \mathrm{C}$ \\
\hline 1 & & $\mathrm{a}$ & & 186.4 \\
\hline 2 & & $141.1^{\mathrm{b}}$ & & 141.3 \\
\hline 3 & $7.66, \mathrm{~s}$ & $117.5^{\mathrm{b}}$ & $7.66, \mathrm{~s}$ & 117.6 \\
\hline 4 & & $183.8^{\mathrm{b}}$ & & 184.0 \\
\hline 5 & & $117.5^{\mathrm{b}}$ & & 117.6 \\
\hline 6 & & $162.6^{\mathrm{b}}$ & & 162.8 \\
\hline 7 & & $\mathrm{a}$ & & 120.9 \\
\hline 8 & & $\mathrm{a}$ & & 175.8 \\
\hline 9 & & $\mathrm{a}$ & & 106.9 \\
\hline 10 & & $128.6^{\mathrm{b}}$ & & 128.8 \\
\hline 11 & & a & & 166.8 \\
\hline 12 & $2.16, \mathrm{~s}$ & $7.9^{\mathrm{b}}$ & $2.16, \mathrm{~s}$ & 8.0 \\
\hline 13 & & 169.7 & & 169.9 \\
\hline 14 & & 129.3 & & 129.5 \\
\hline 15 & 6.46, br d (11.2) & 138.0 & $6.46, \mathrm{dd}(11.4,1.6)$ & 138.2 \\
\hline 16 & $6.78, \mathrm{dd}(15.2,11.2)$ & 127.3 & $6.78, \mathrm{dd}(15.1,11.1)$ & 127.5 \\
\hline 17 & $6.02, \mathrm{dd}(15.2,8.0)$ & 145.4 & $6.02, \mathrm{dd}(15.1,7.9)$ & 145.6 \\
\hline 18 & $2.42, \operatorname{ddq}(9.1,8.0,6.9)$ & 42.0 & 2.43 , ddq $(9.2,7.6,6.8)$ & 42.3 \\
\hline 19 & $3.77, \mathrm{dd}(9.1,1.4)$ & 75.5 & $3.78, \mathrm{dd}(9.1,1.8)$ & 75.6 \\
\hline 20 & $1.86-1.89, \mathrm{~m}$ & 35.8 & $1.75-2.05, \mathrm{~m}(1$ of $7 \mathrm{H})$ & 36.0 \\
\hline 21 & $3.51, \mathrm{dd}(8.3,4.3)$ & 77.9 & $3.51, \mathrm{dd}(8.4,2.9)$ & 78.2 \\
\hline 22 & $1.83-1.89, \mathrm{~m}$ & 36.7 & $1.75-2.05, \mathrm{~m}(1$ of $7 \mathrm{H})$ & 36.9 \\
\hline 23 & $3.95, \mathrm{dd}(10.6,1.1)$ & 74.7 & $3.95, \mathrm{dd}(10.6,1.7)$ & 75.0 \\
\hline 24 & $1.97-2.01, \mathrm{~m}$ & 35.0 & $1.75-2.05, \mathrm{~m}(1$ of $7 \mathrm{H})$ & 35.2 \\
\hline 25 & $4.22, \mathrm{dd}(6.7,3.4)$ & 81.3 & $4.22, \mathrm{dd}(6.7,3.4)$ & 81.5 \\
\hline $26 \mathrm{a}$ & $1.89-1.93, \mathrm{~m}$ & 24.6 & $1.75-2.05, \mathrm{~m}(1$ of $7 \mathrm{H})$ & 24.8 \\
\hline $26 \mathrm{~b}$ & $1.93-1.97, \mathrm{~m}$ & & $1.75-2.05, \mathrm{~m}(1$ of $7 \mathrm{H})$ & \\
\hline $27 \mathrm{a}$ & $1.78-1.83, \mathrm{~m}$ & 34.9 & $1.75-2.05, \mathrm{~m}(1$ of $7 \mathrm{H})$ & 35.1 \\
\hline $27 b$ & $2.01-2.05, \mathrm{~m}$ & & $1.75-2.05, \mathrm{~m}(1$ of $7 \mathrm{H})$ & \\
\hline 28 & & 106.2 & & 106.4 \\
\hline 29 & $2.08, \mathrm{~s}$ & 20.3 & $2.08, \mathrm{~s}$ & 20.5 \\
\hline 30 & $0.99, \mathrm{~d}(6.9)$ & 16.7 & $0.99, \mathrm{~d}(6.8)$ & 16.9 \\
\hline 31 & $1.01, \mathrm{~d}(7.2)$ & 10.9 & $1.01, \mathrm{~d}(7.0)$ & 11.1 \\
\hline 32 & $0.89, \mathrm{~d}(7.0)$ & 10.1 & $0.89, \mathrm{~d}(6.9)$ & 10.3 \\
\hline 33 & $1.39, \mathrm{~s}$ & 24.0 & $1.39, \mathrm{~s}$ & 24.1 \\
\hline 34 & $0.73, \mathrm{~d}(6.9)$ & 12.6 & $0.72, \mathrm{~d}(6.9)$ & 12.8 \\
\hline
\end{tabular}

${ }^{\mathrm{a}}$ Shifts not determined due to small amount of material. ${ }^{\mathrm{b}}$ Shifts determined from HMBC. 
${ }^{1} \mathrm{H}$ NMR spectrum of isolated rifsaliniketal 9 in $\mathrm{CD}_{3} \mathrm{OD}$

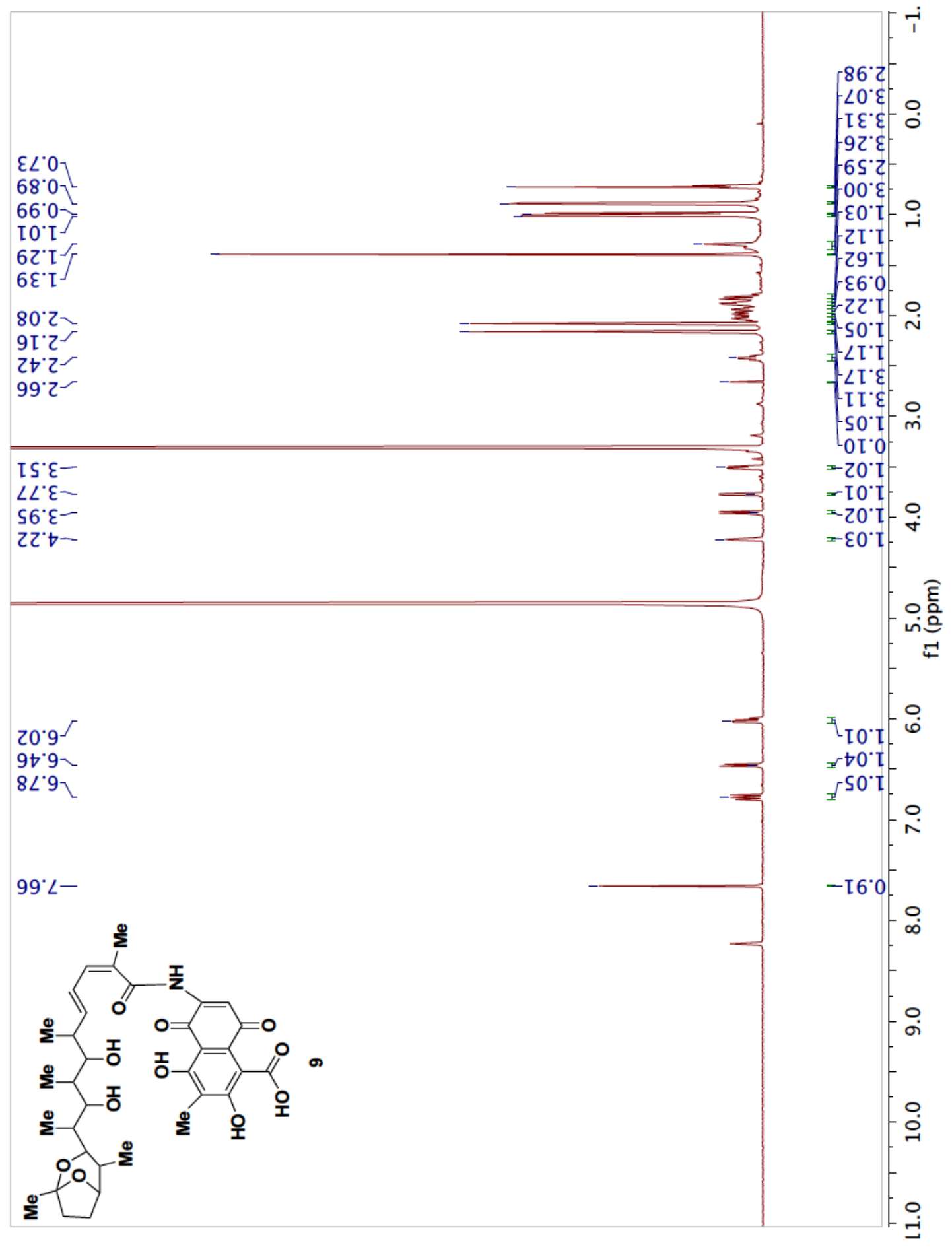


${ }^{13} \mathrm{CNMR}$ spectrum of isolated rifsaliniketal 9 in $\mathrm{CD}_{3} \mathrm{OD}$

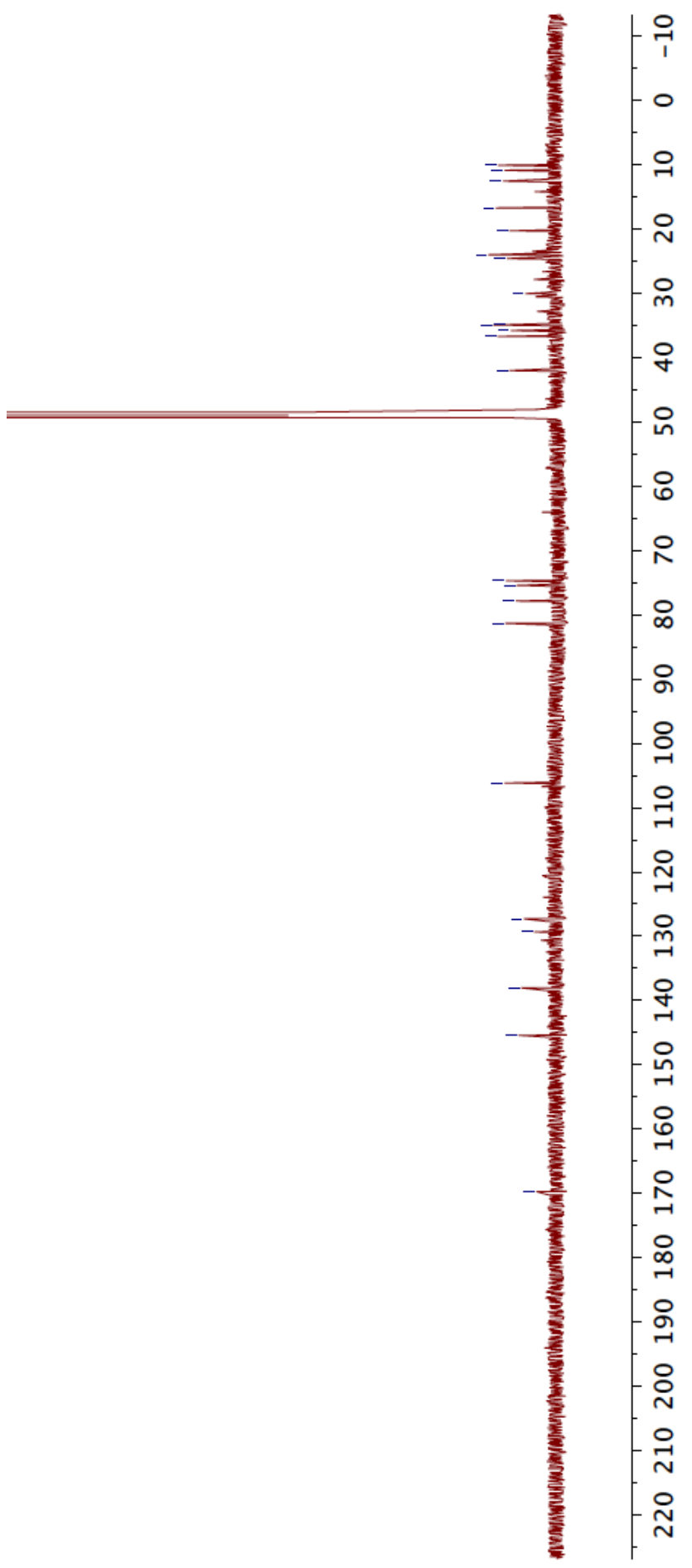


COSY spectrum of isolated rifsaliniketal 9 in $\mathrm{CD}_{3} \mathrm{OD}$

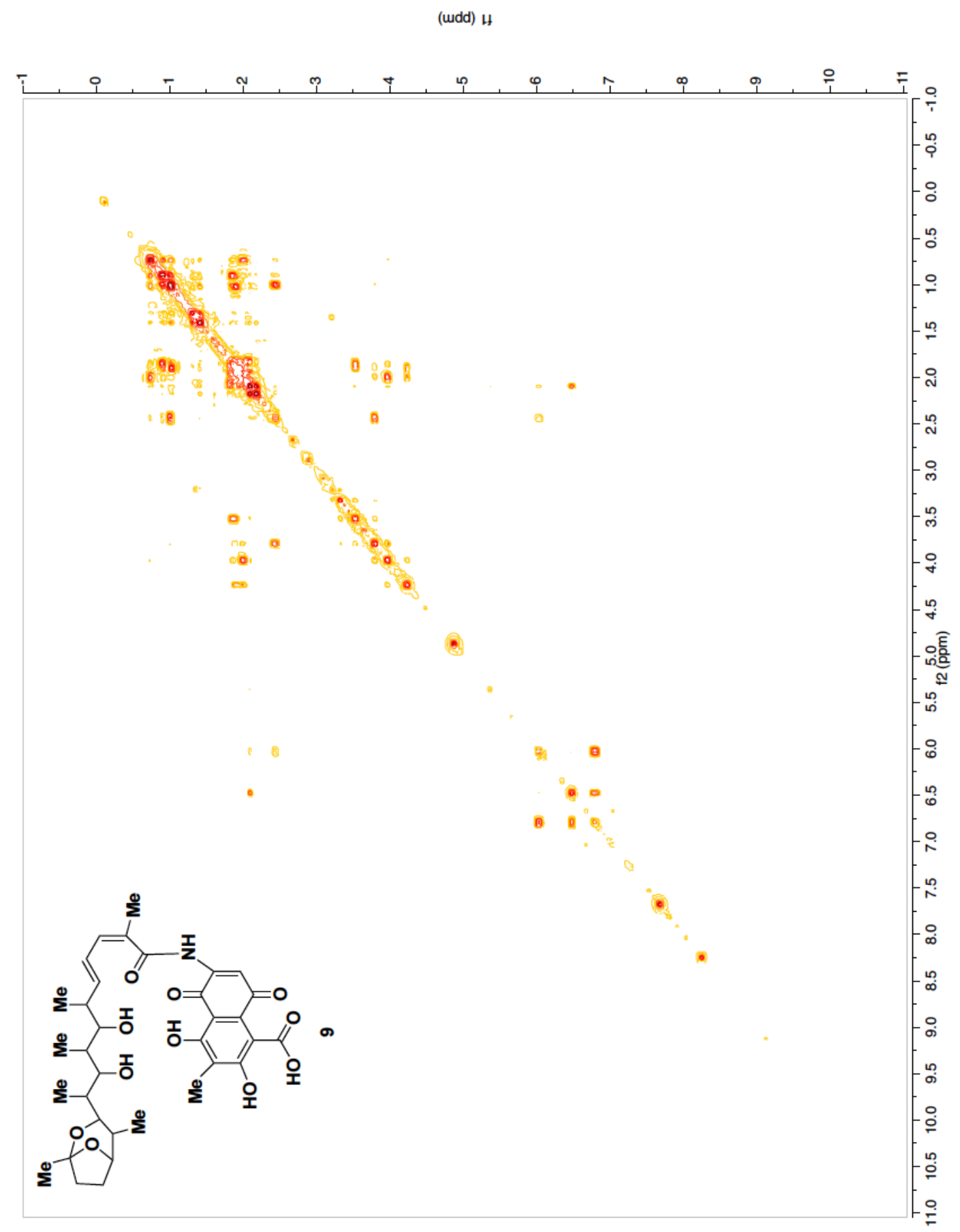


$\mathrm{HSQC}$ spectrum of isolated rifsaliniketal 9 in $\mathrm{CD}_{3} \mathrm{OD}$

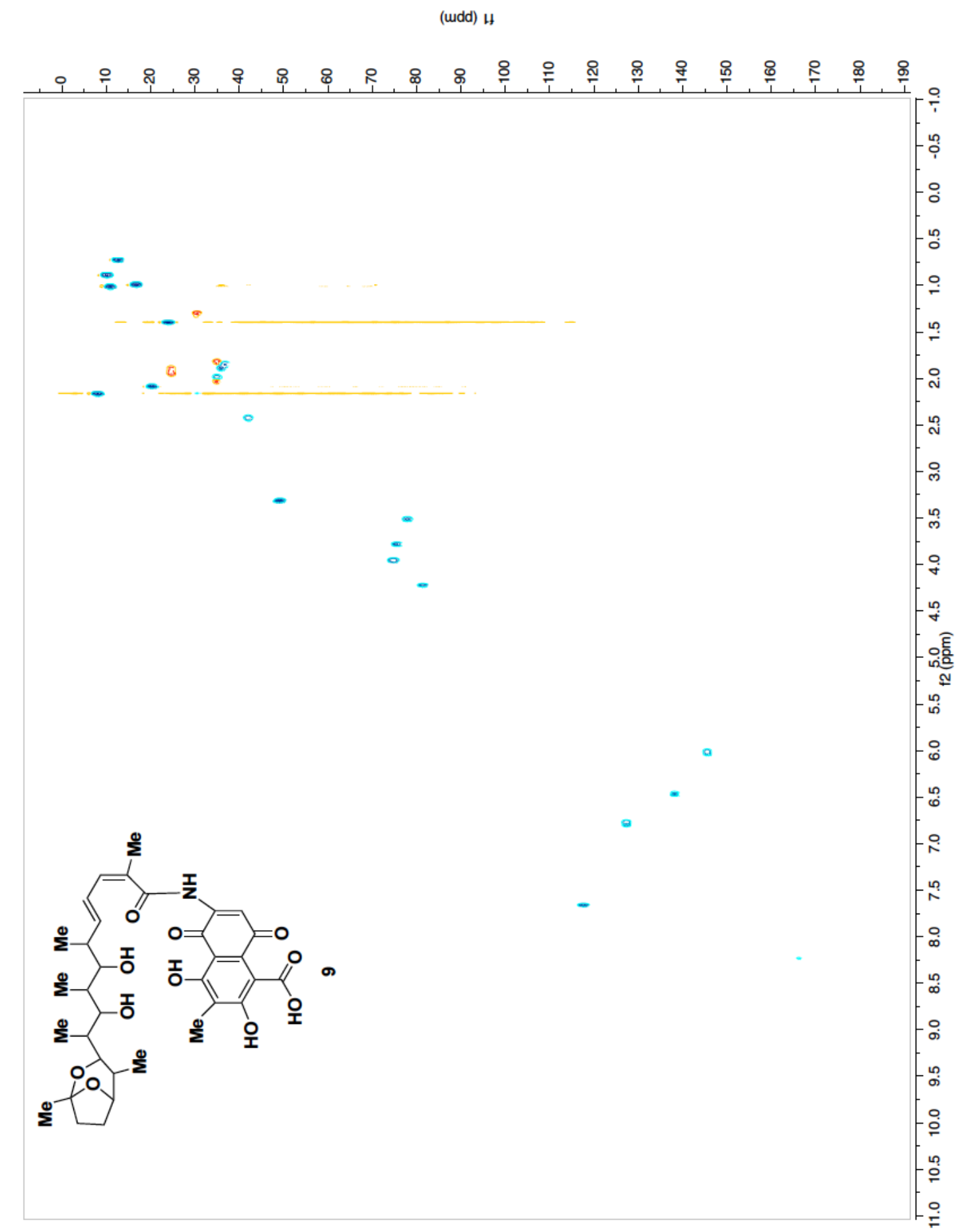


$\mathrm{HMBC}$ spectrum of isolated rifsaliniketal 9 in $\mathrm{CD}_{3} \mathrm{OD}$

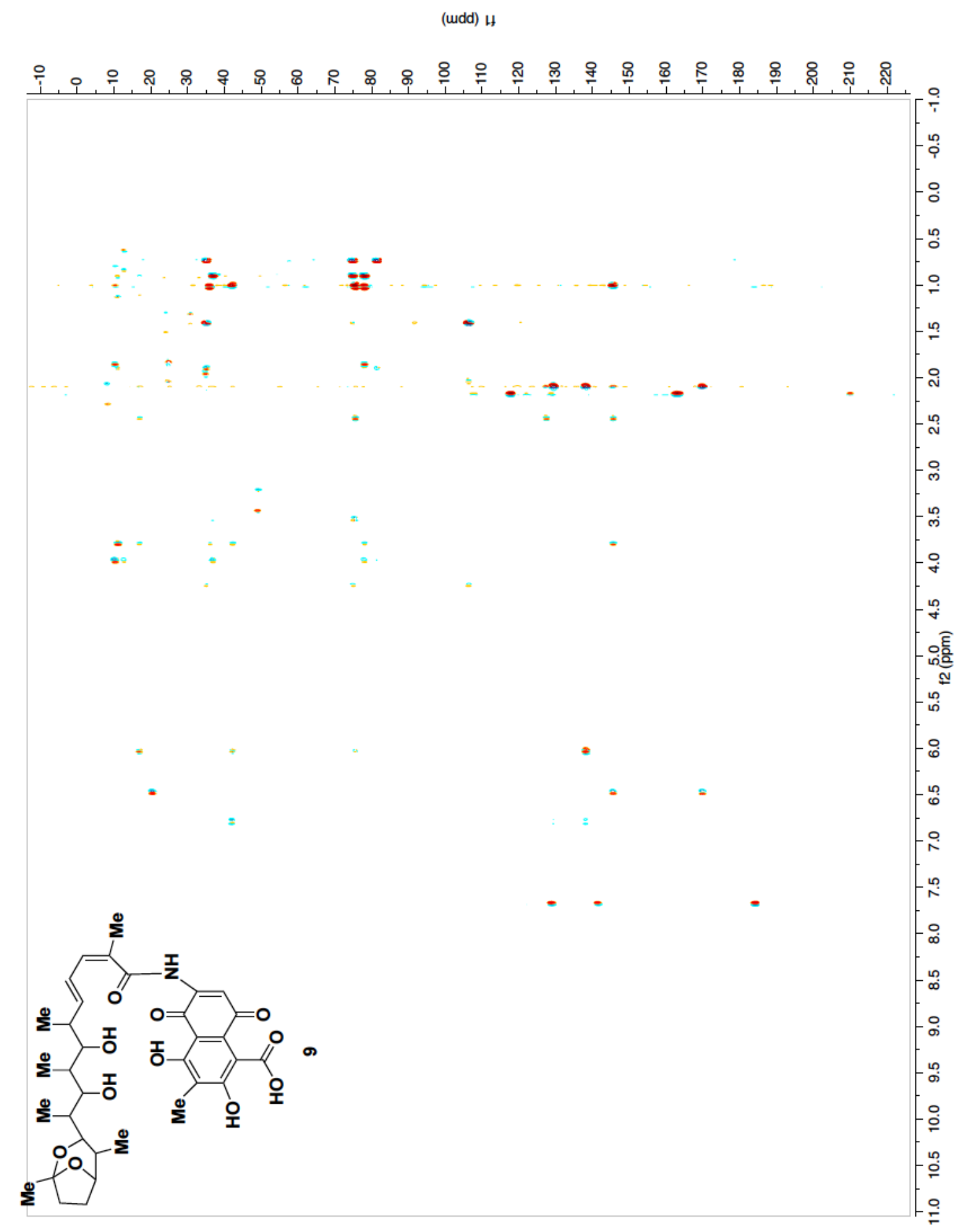


${ }^{1} \mathrm{H}$ spectrum of rifsaliniketal methyl ester $9 \mathrm{a}$ in $\mathrm{CD}_{3} \mathrm{OD}$

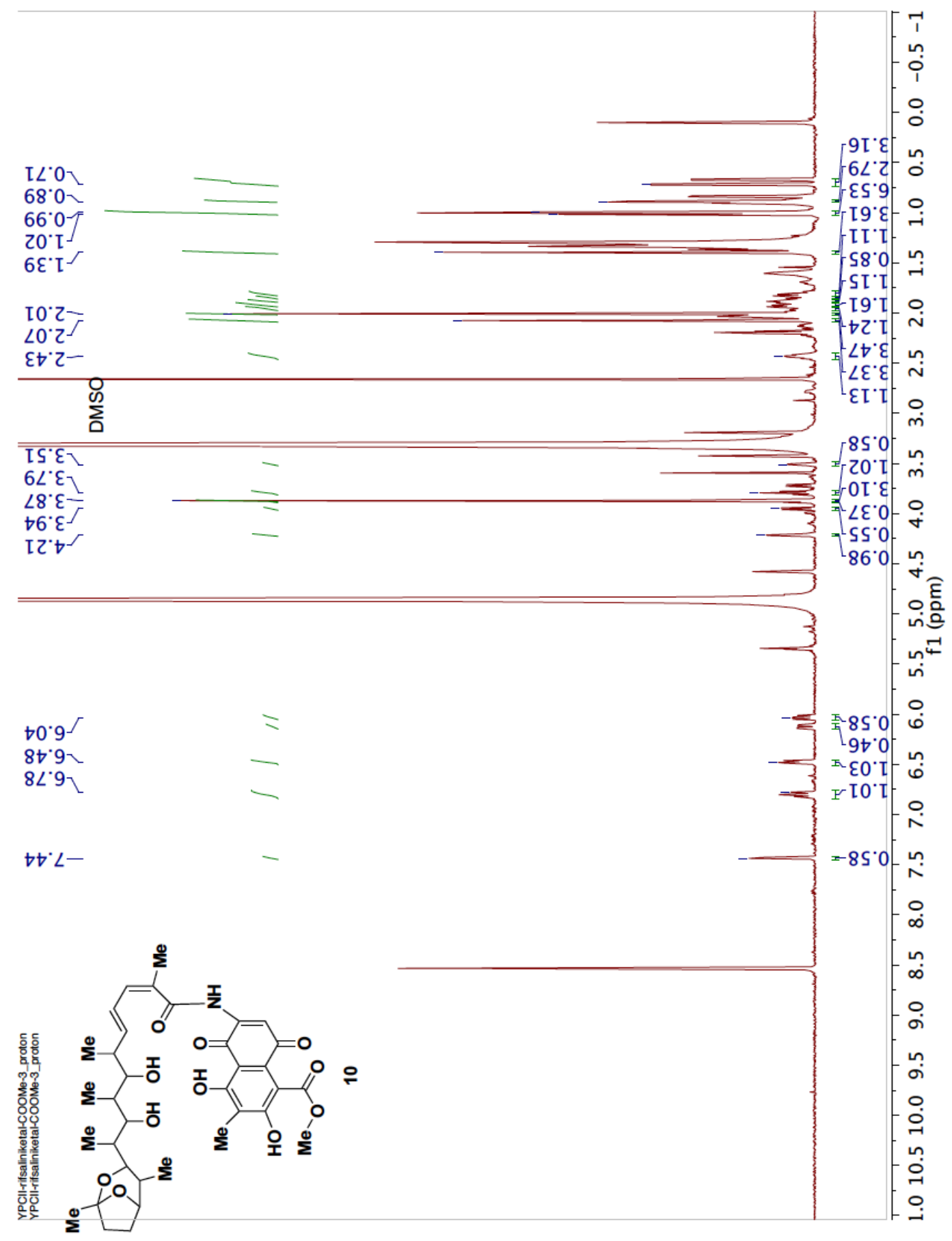


$\mathrm{HMBC}$ spectrum of rifsaliniketal methyl ester $9 \mathrm{a}$ in $\mathrm{CD}_{3} \mathrm{OD}$

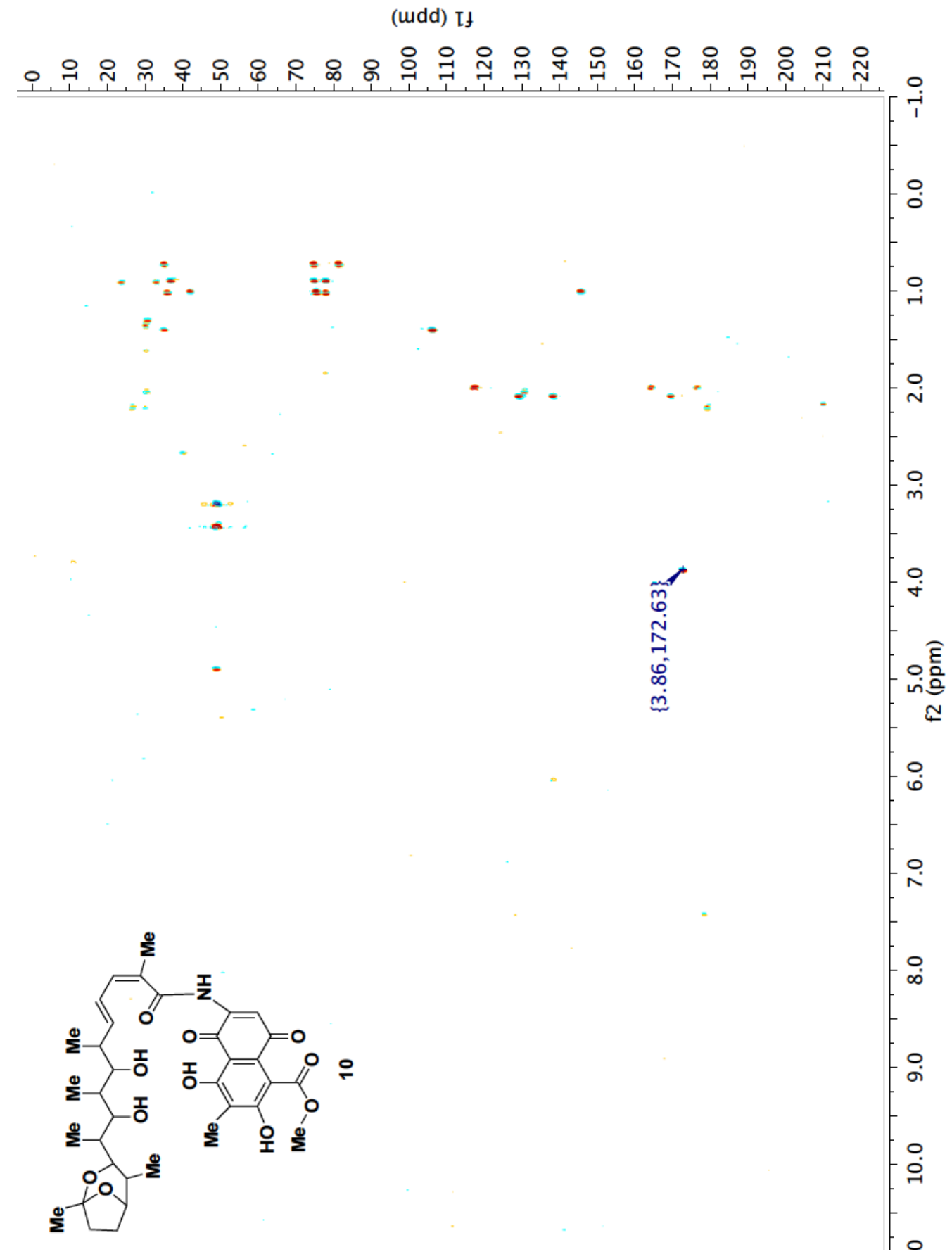




\section{$\mathrm{H}$ and $\mathrm{C}-\mathrm{NMR}\left(\mathrm{CDCl}_{3}\right)$ of compound 17}
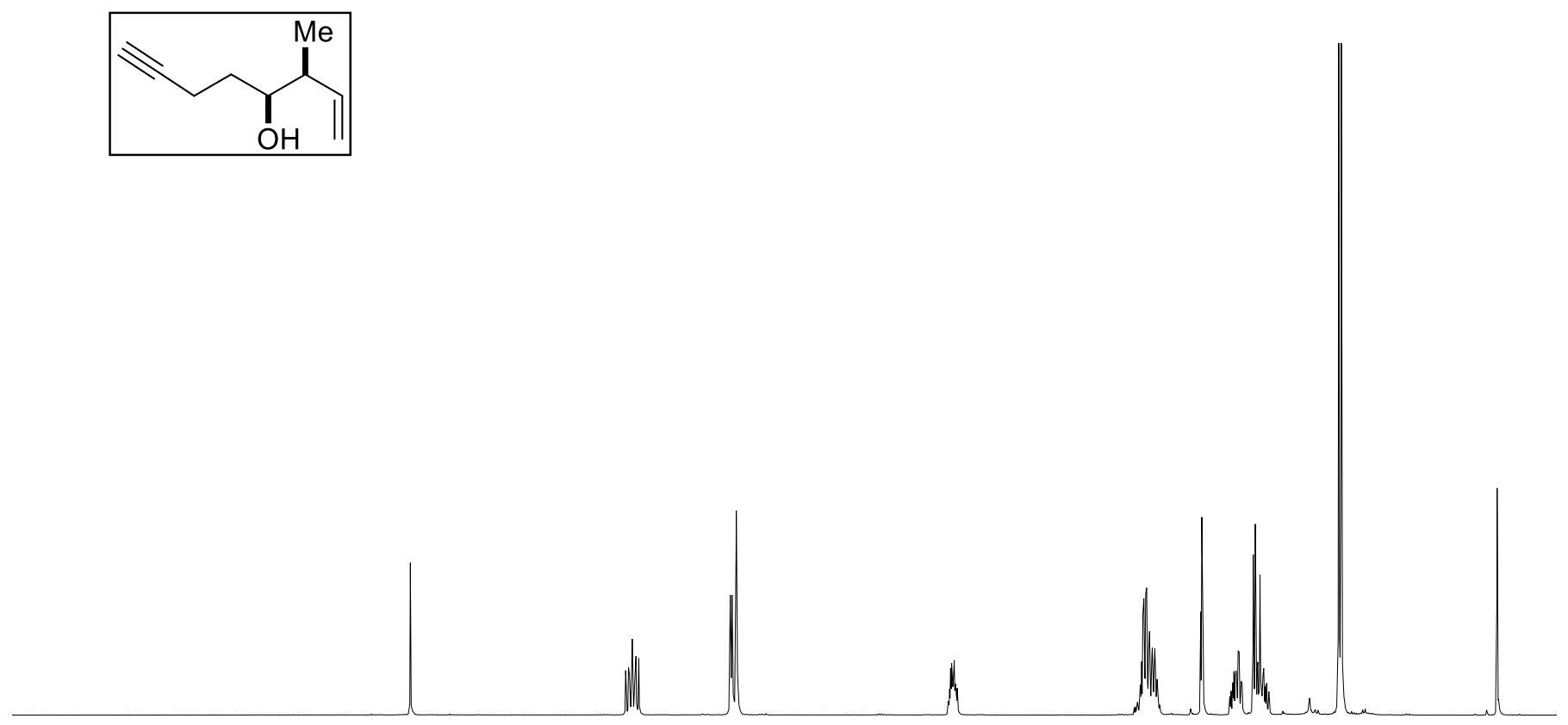

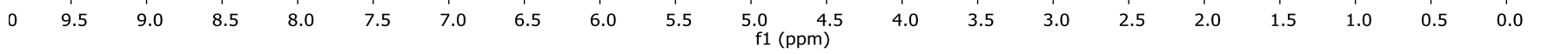

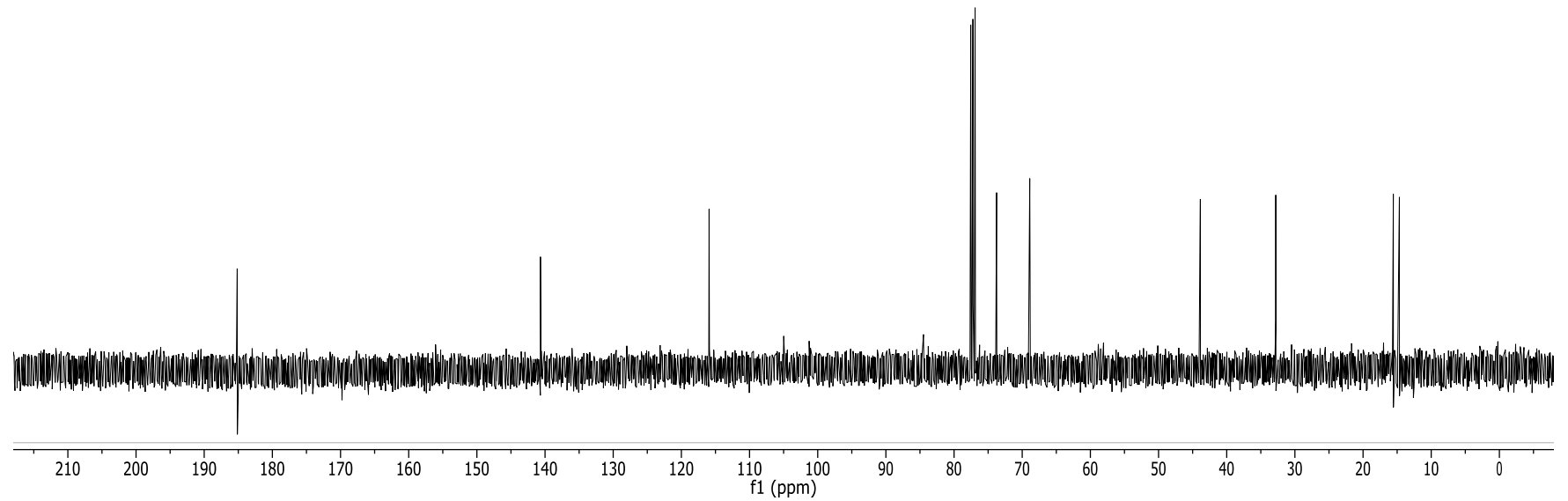




\section{$\mathrm{H}$ and $\mathrm{C}-\mathrm{NMR}\left(\mathrm{CDCl}_{3}\right)$ of compound S1}
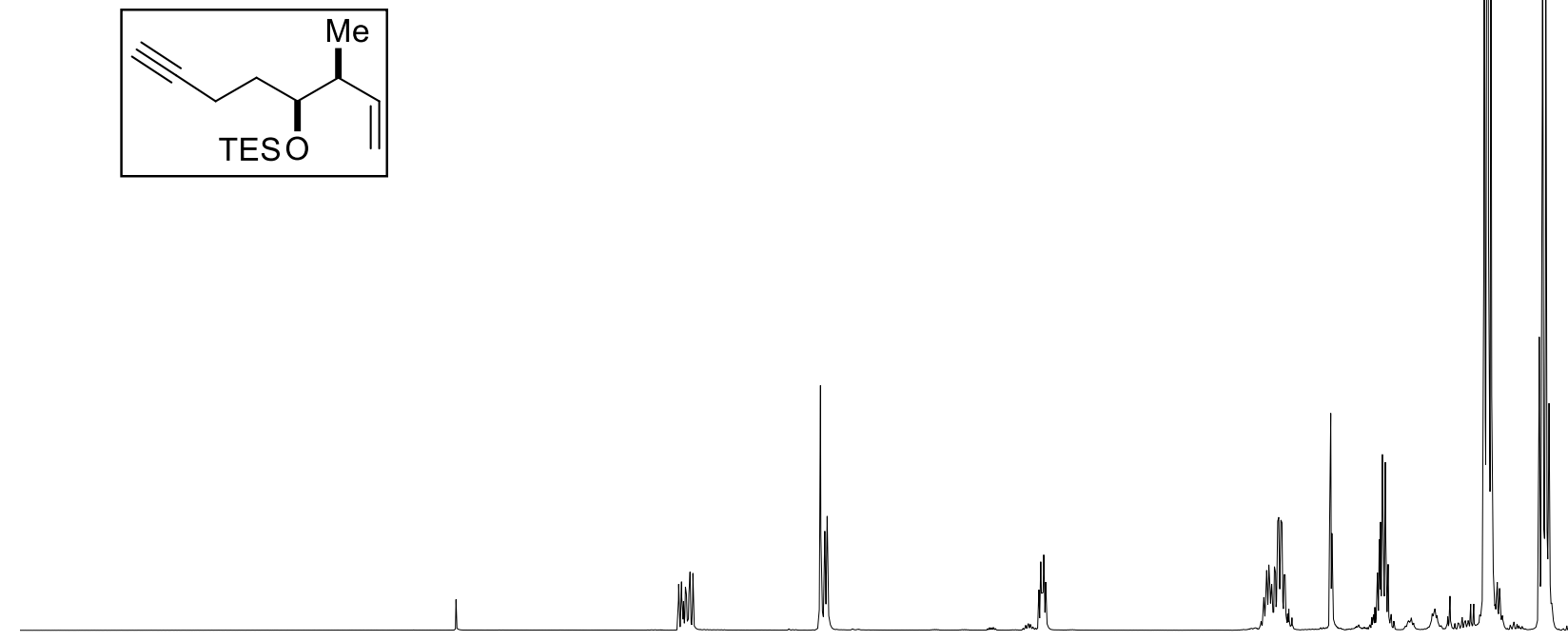

\begin{tabular}{|c|c|c|c|c|c|c|c|c|c|c|c|c|c|c|c|c|c|c|}
\hline 9.5 & 9.0 & 8.5 & 8.0 & 7.5 & 7.0 & 6.5 & 6.0 & 5.5 & $\begin{array}{l}5.0 \\
\mathrm{f} 1(\mathrm{ppm})\end{array}$ & 4.0 & 3.5 & 3.0 & 2.5 & 2.0 & 1.5 & 1.0 & 0.5 & 0.0 \\
\hline
\end{tabular}
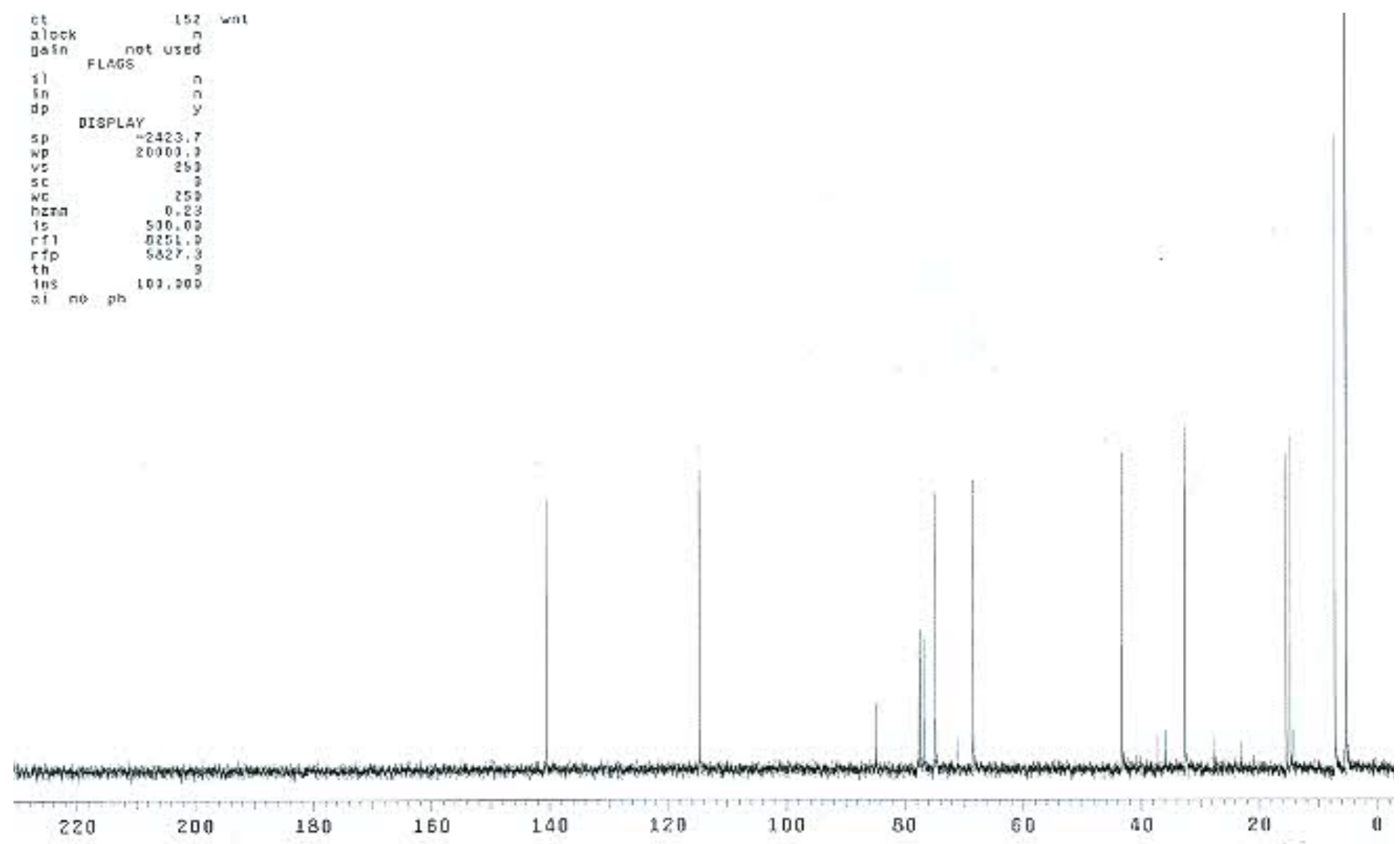


\section{$\mathrm{H}$ and C-NMR $\left(\mathrm{CDCl}_{3}\right)$ of compound 13}
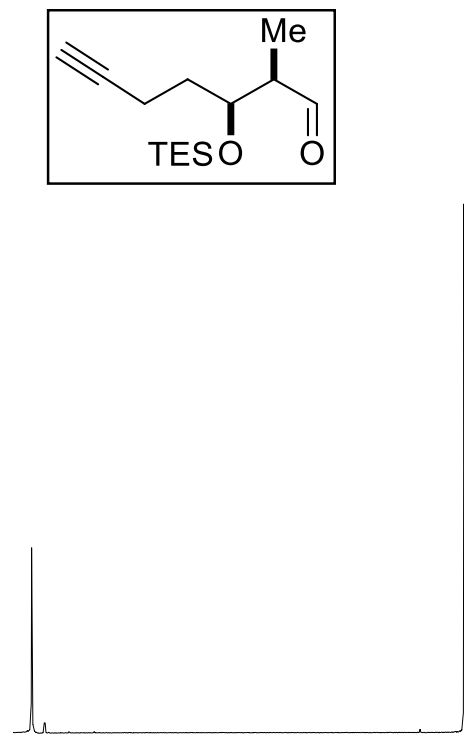

$M$
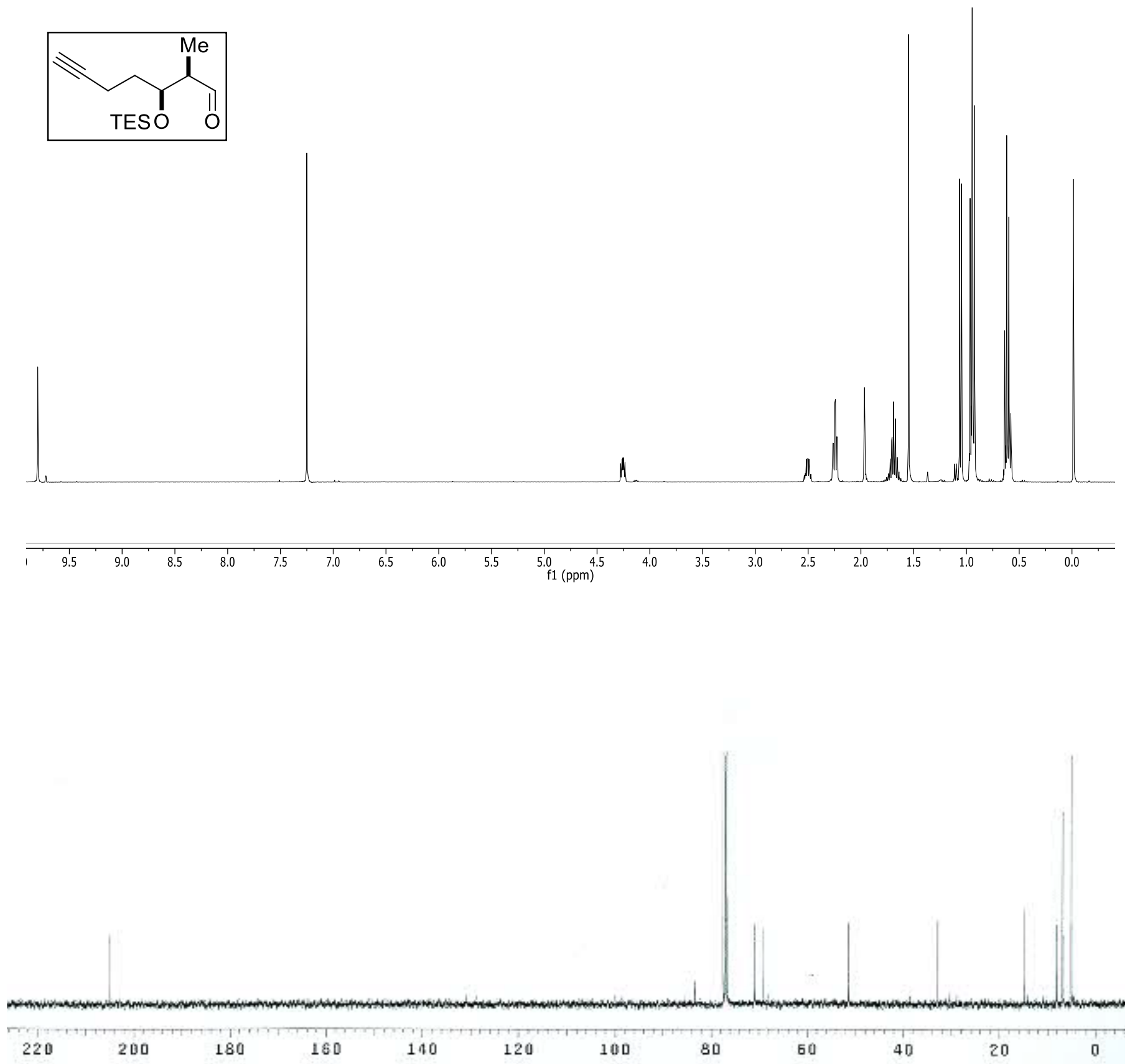


\section{$\mathrm{H}$ and $\mathrm{C}-\mathrm{NMR}\left(\mathrm{CDCl}_{3}\right)$ of compound 18 -syn}
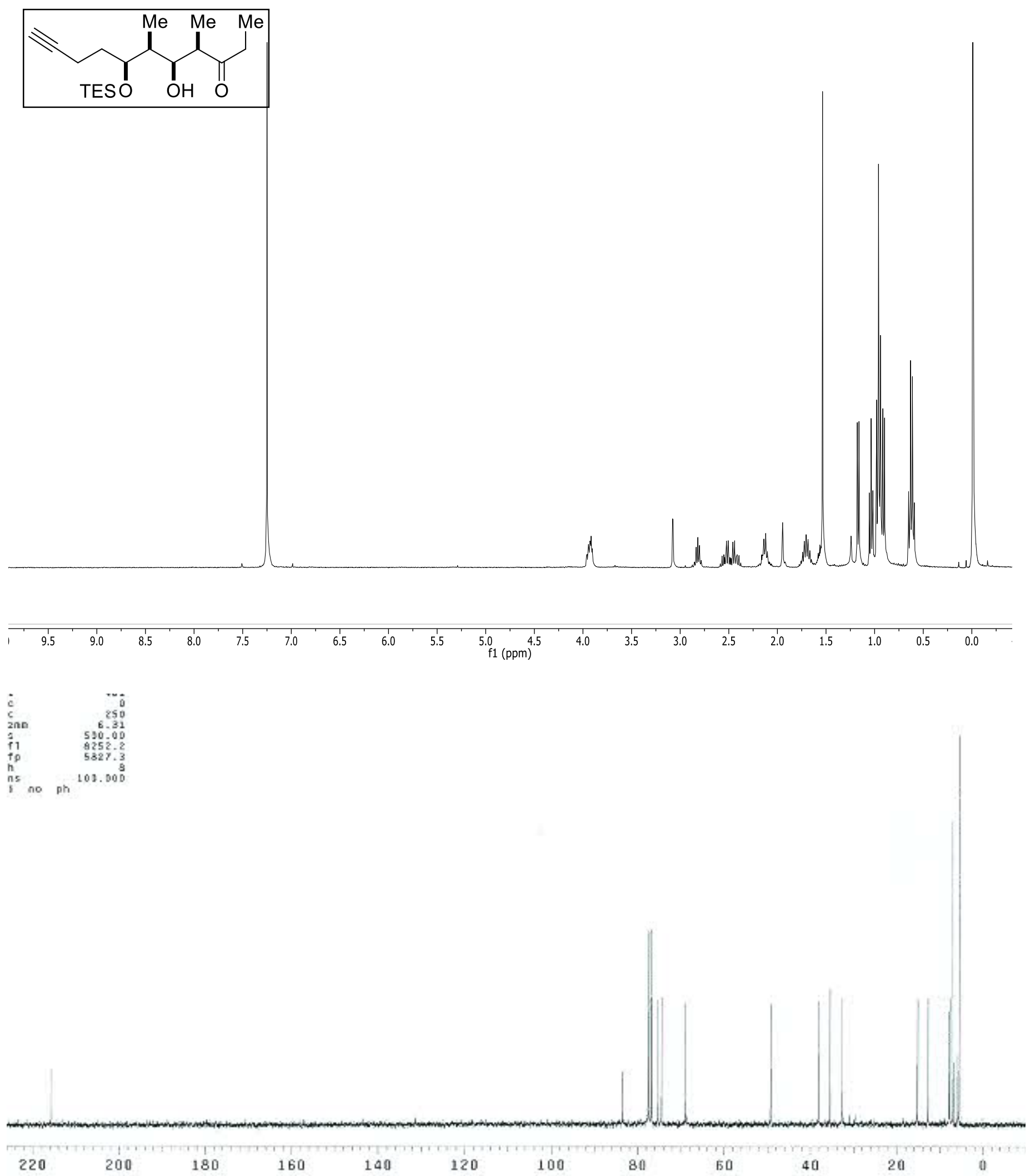


\section{H- NMR $\left(\mathrm{CDCl}_{3}\right)$ of compound 19}
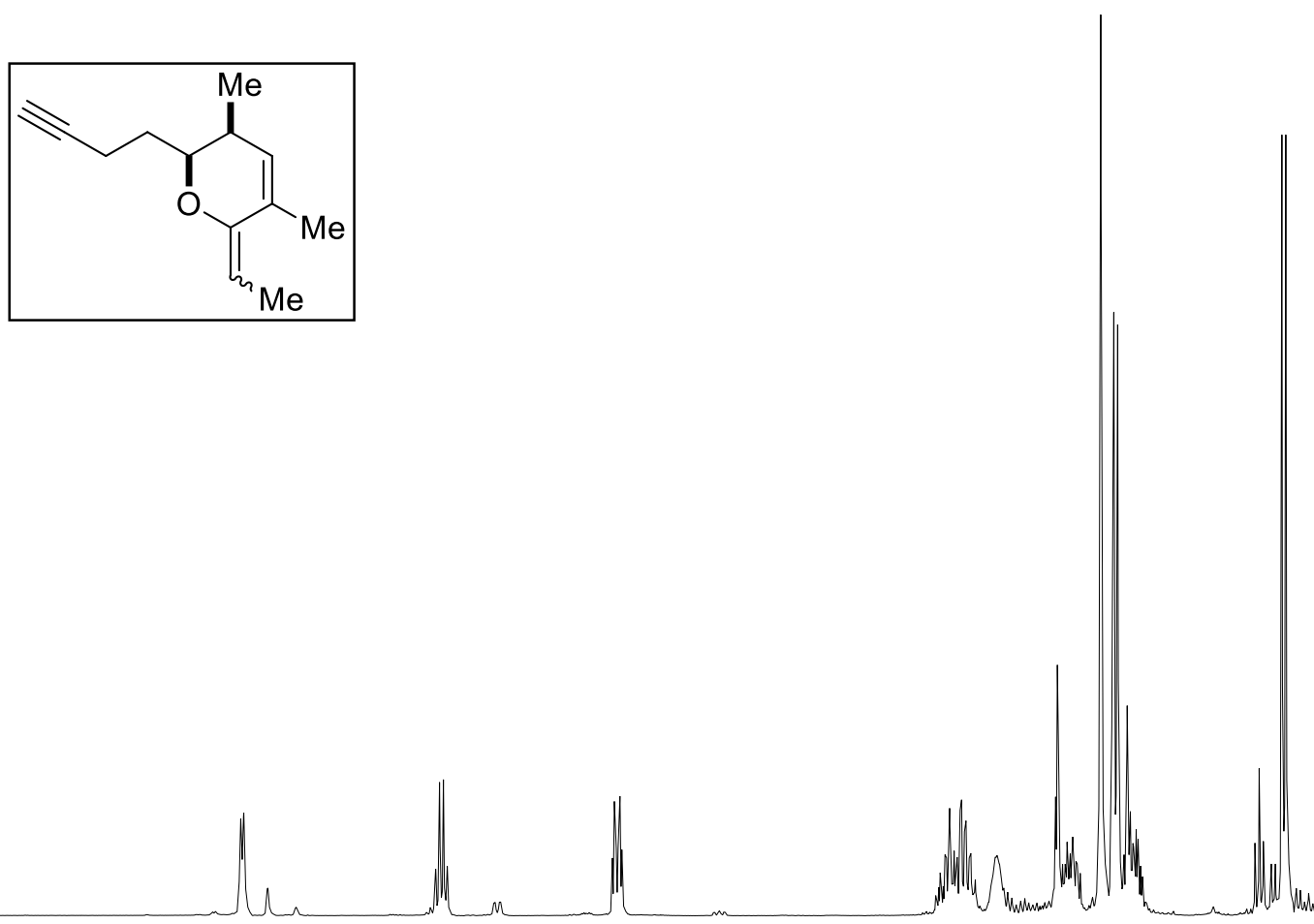

\begin{tabular}{|c|c|c|c|c|c|c|c|c|c|c|c|c|c|c|}
\hline 7.5 & 7.0 & 6.5 & 6.0 & 5.5 & $\mathrm{f}^{5}(\mathrm{ppm})$ & 4.0 & 3.5 & 3.0 & 2.5 & 2.0 & 1.5 & 1.0 & 0.5 & 0.0 \\
\hline
\end{tabular}


$\mathrm{H}$ and C-NMR ( $\left.\mathrm{CDCl}_{3}\right)$ of compound 21-syn
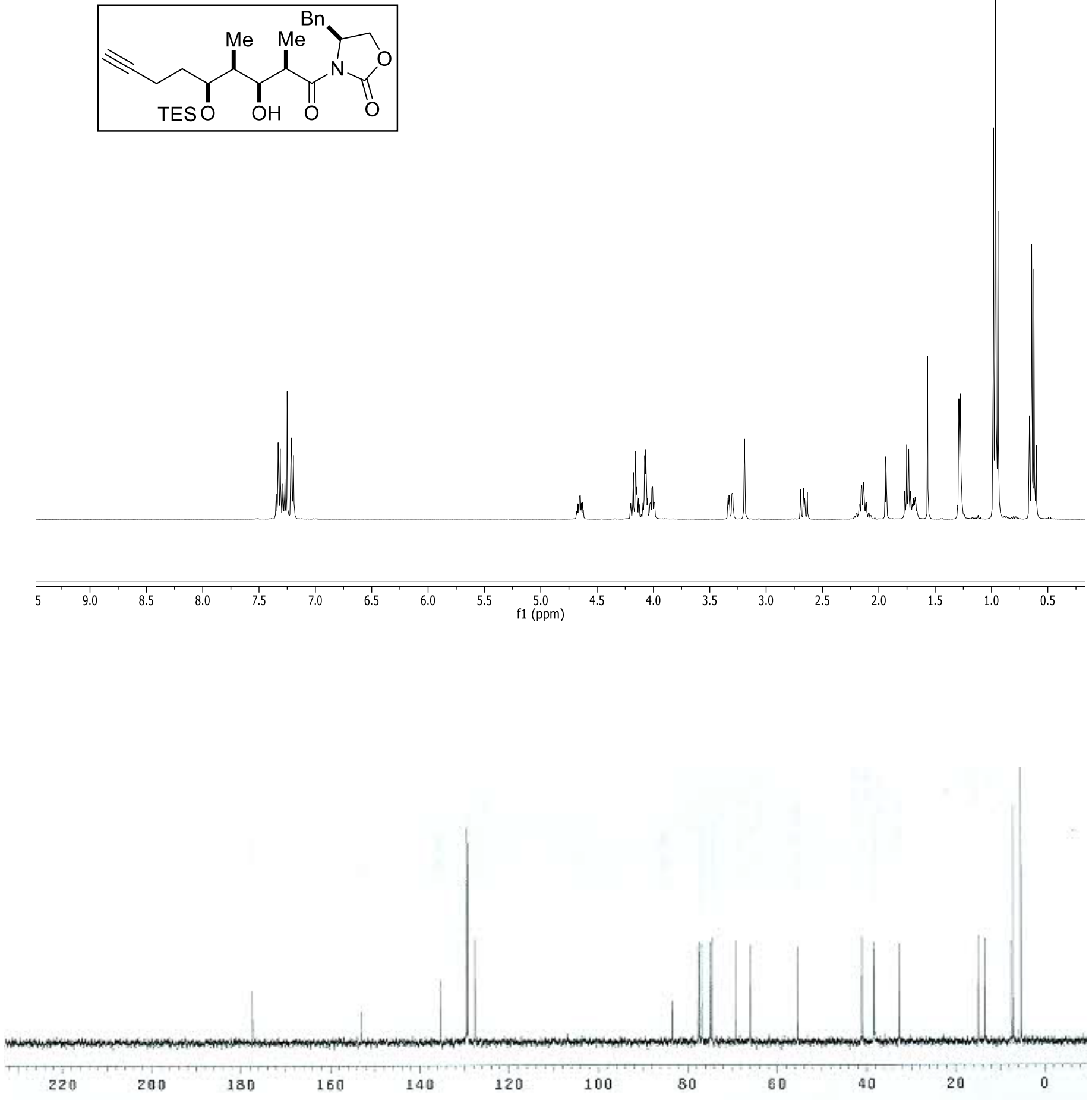
$\mathrm{H}$ and C-NMR $\left(\mathrm{CDCl}_{3}\right)$ of compound 21-anti
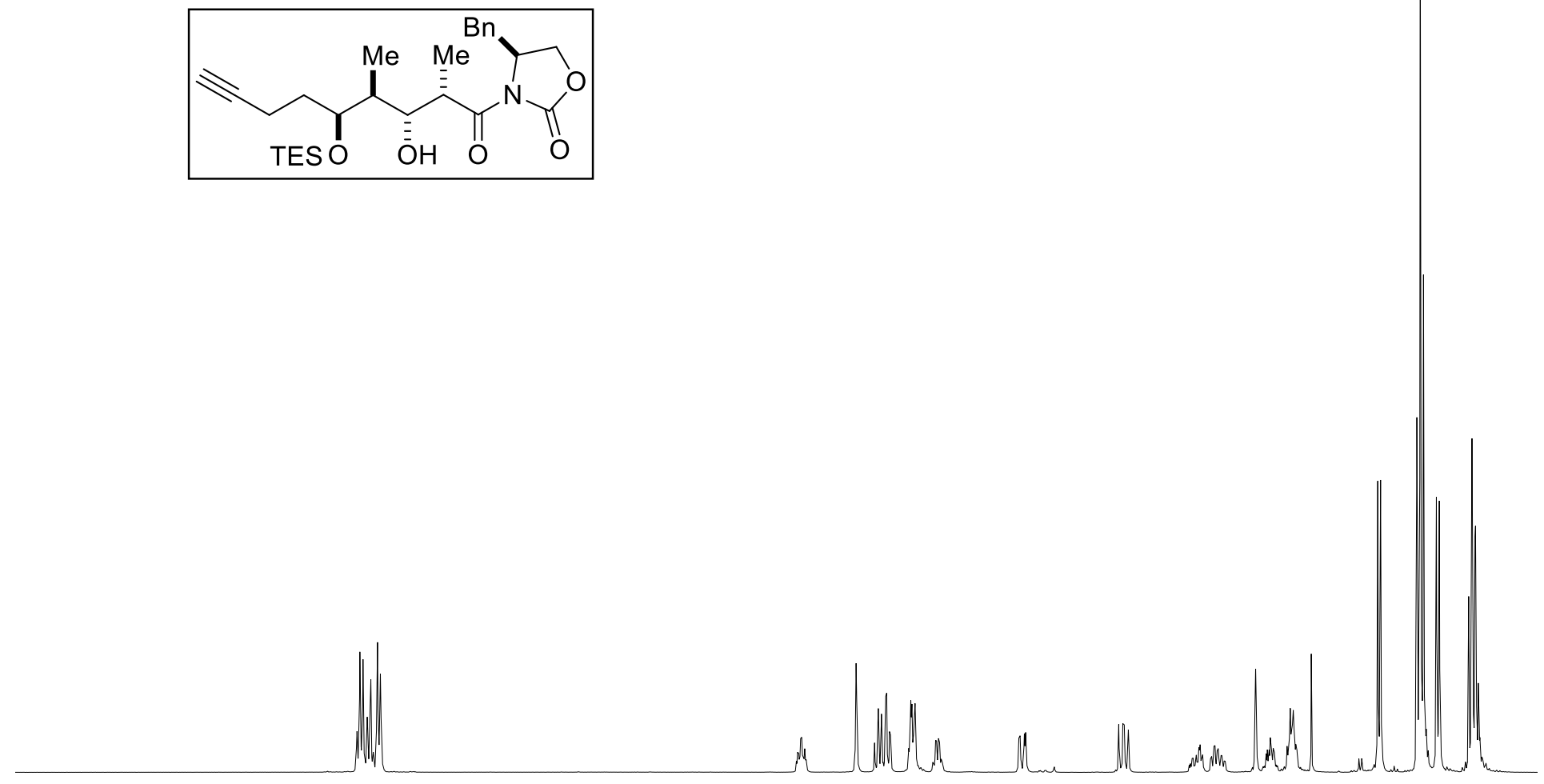

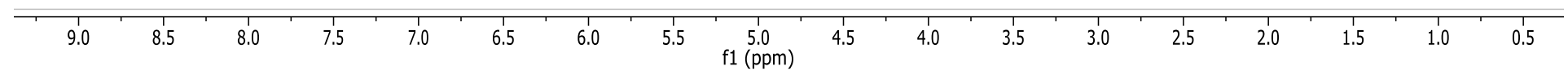


$\mathrm{H}$ and C-NMR $\left(\mathrm{CDCl}_{3}\right)$ of compound 23-anti
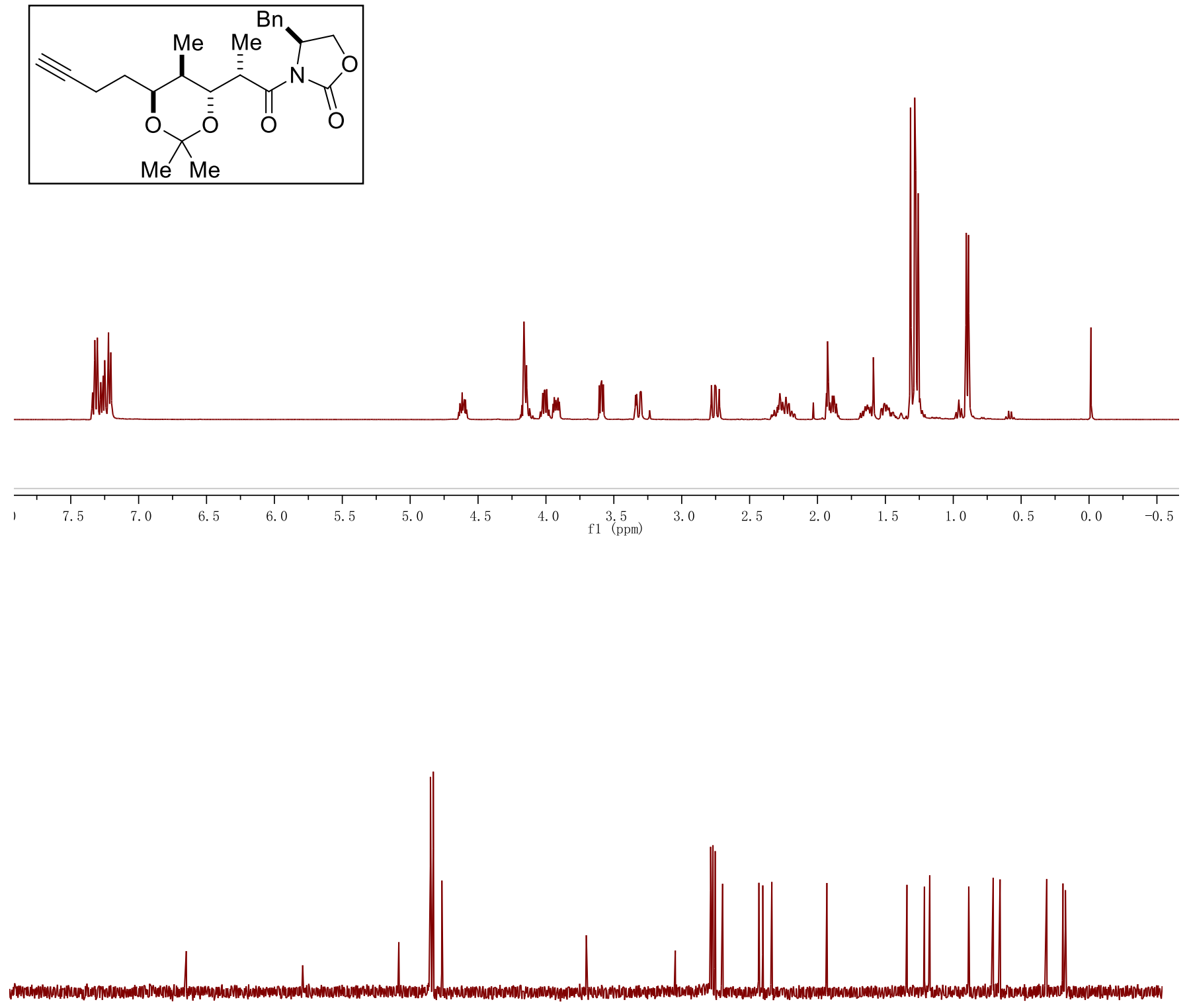

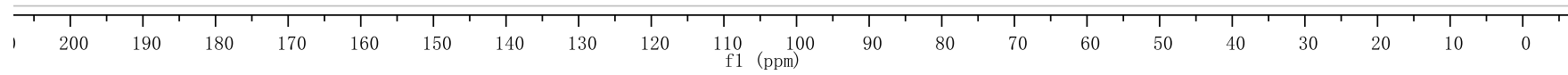


$\mathrm{H}$ and C-NMR ( $\left.\mathrm{CDCl}_{3}\right)$ of compound 23-syn
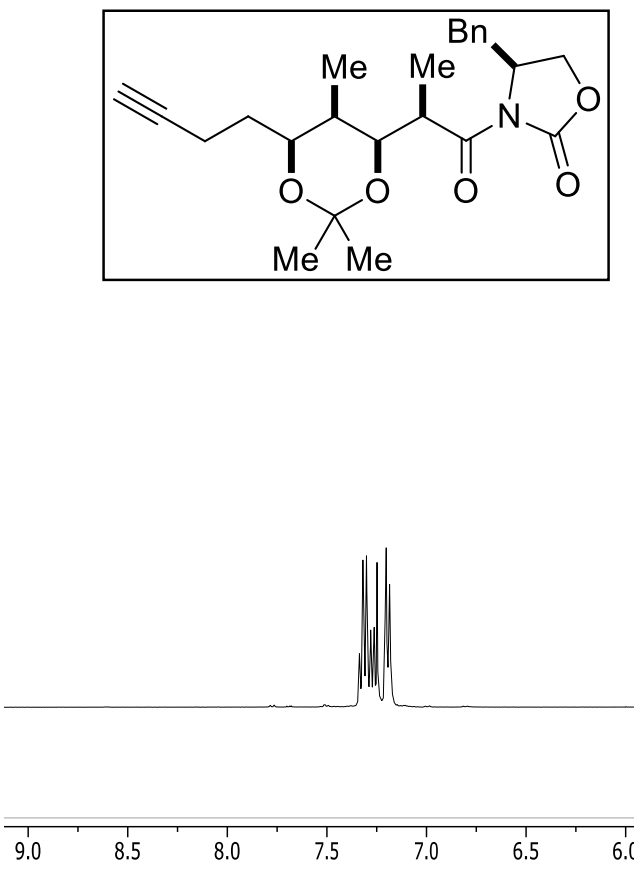

$\begin{array}{llc}5.5 & 5.0 \\ & f 1(\mathrm{ppm})\end{array}$

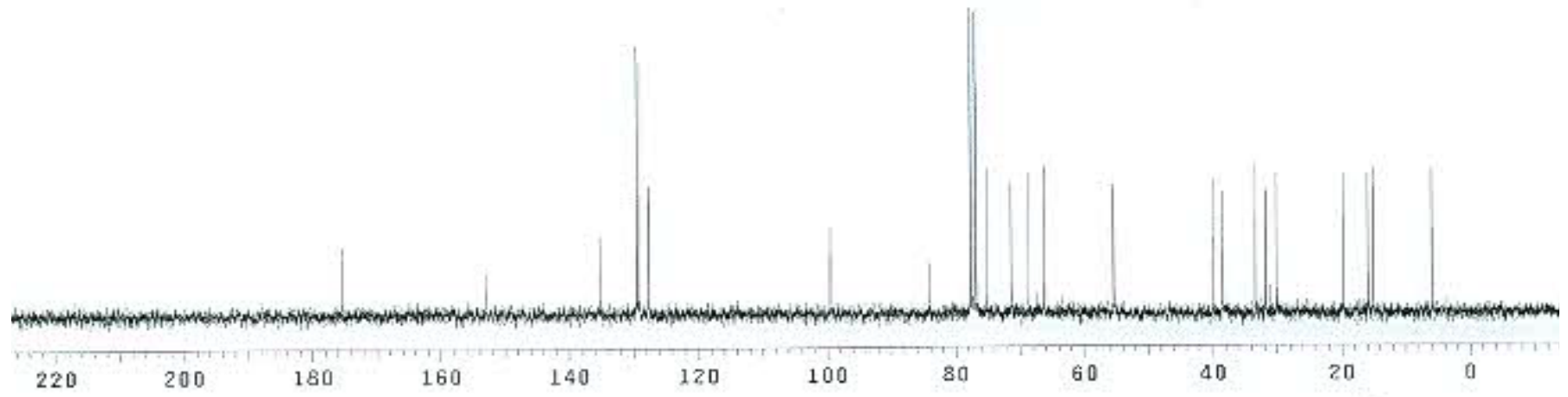




\section{H and C-NMR $\left(\mathrm{CDCl}_{3}\right)$ of compound 26}
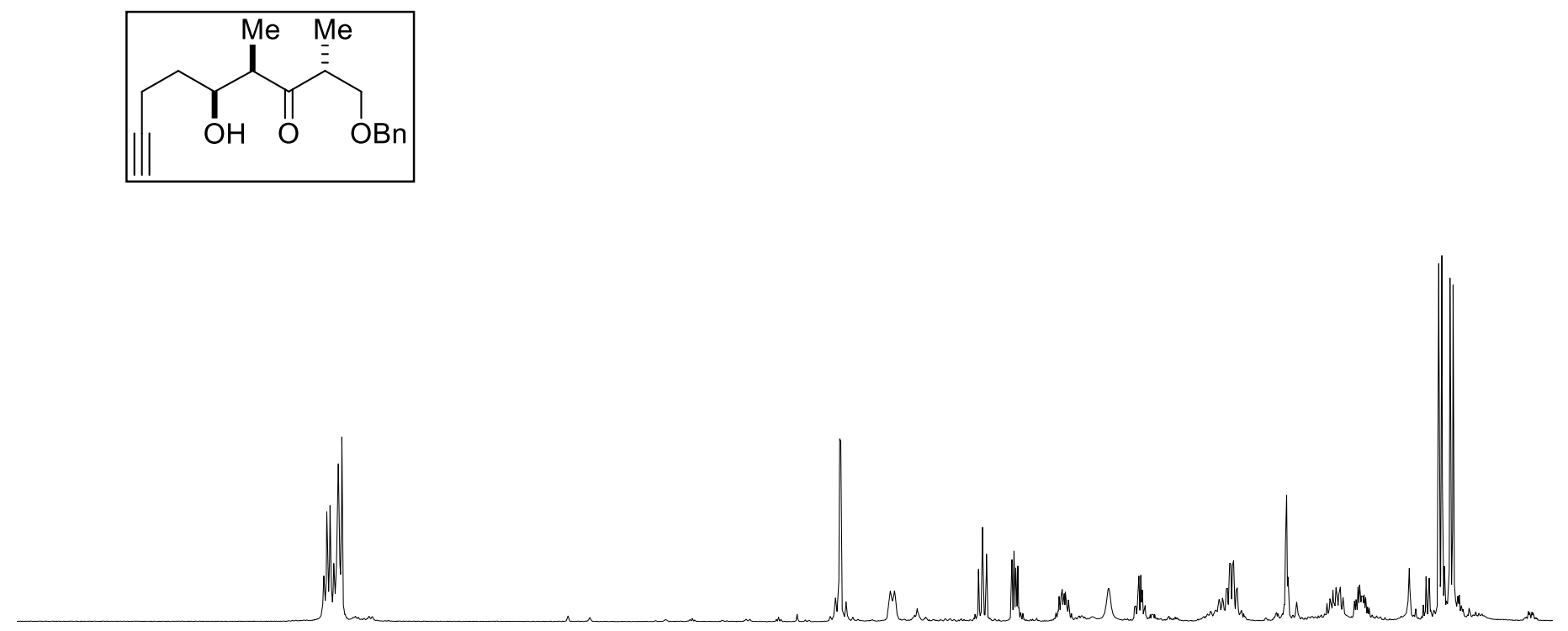

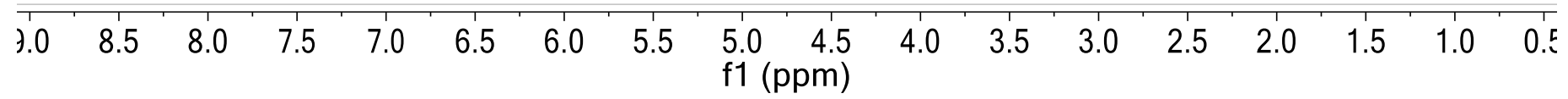

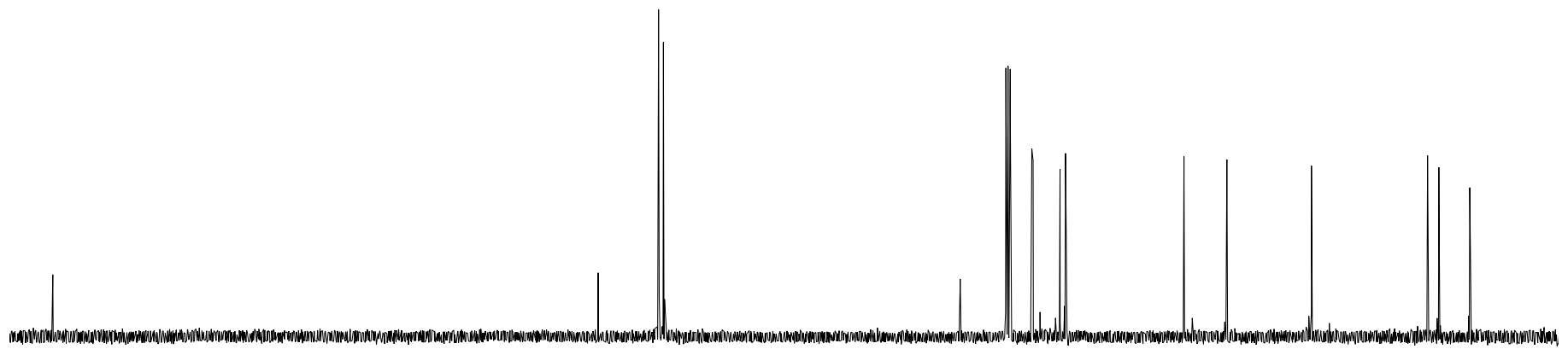

$\begin{array}{lllllllllllllllllllllll}220 & 210 & 200 & 190 & 180 & 170 & 160 & 150 & 140 & 130 & \begin{array}{c}120 \\ \mathrm{f} 1\end{array}\left(\begin{array}{lllllll}1 \\ (\mathrm{ppm})\end{array}\right. & 100 & 90 & 80 & 70 & 60 & 50 & 40 & 30 & 20 & 10 & 0\end{array}$ 
H-NMR $\left(\mathrm{CDCl}_{3}\right)$ and C-NMR $\left(\mathrm{CD}_{3} \mathrm{OD}\right)$ of compound 31-syn
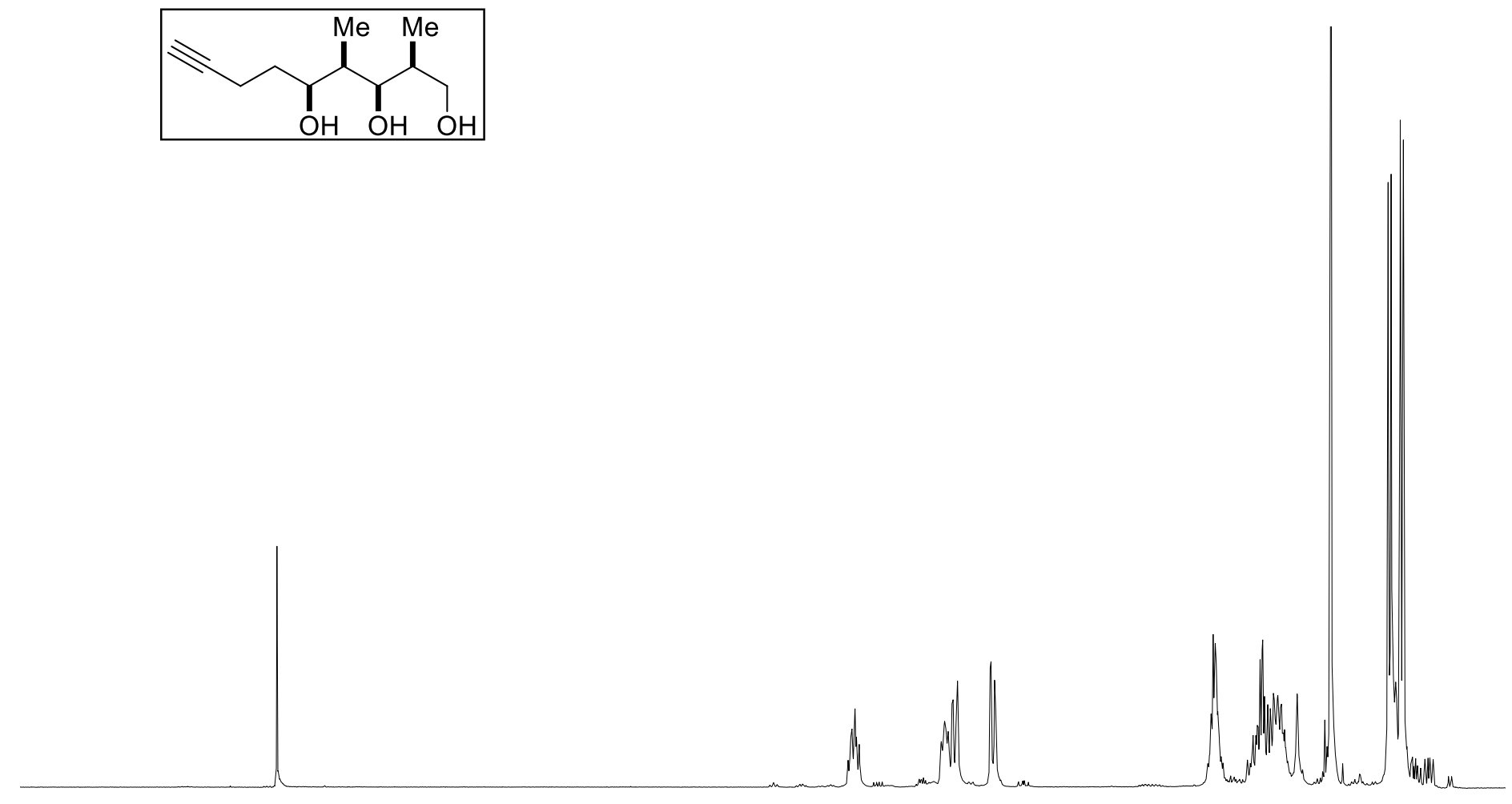

\begin{tabular}{|c|c|c|c|c|c|c|c|c|c|c|c|c|c|c|c|c|}
\hline 8.5 & 8.0 & 7.5 & 7.0 & 6.5 & 6.0 & 5.5 & $\begin{array}{l}5.0 \\
\mathrm{f} 1(\mathrm{ppm})\end{array}$ & ${ }^{\prime} \cdot{ }^{\prime}$ & 4.0 & 3.5 & 3.0 & 2.5 & 2.0 & 1.5 & 1.0 & $\overline{0.5}$ \\
\hline
\end{tabular}

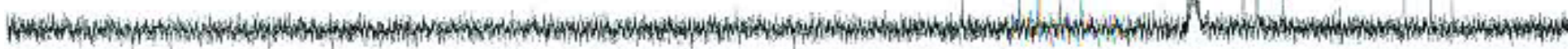

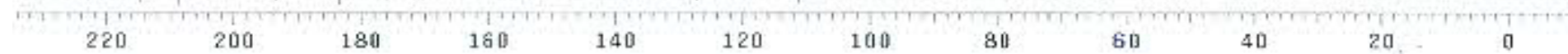




\section{$\mathrm{H}$ and C-NMR $\left(\mathrm{CDCl}_{3}\right)$ of compound 32}
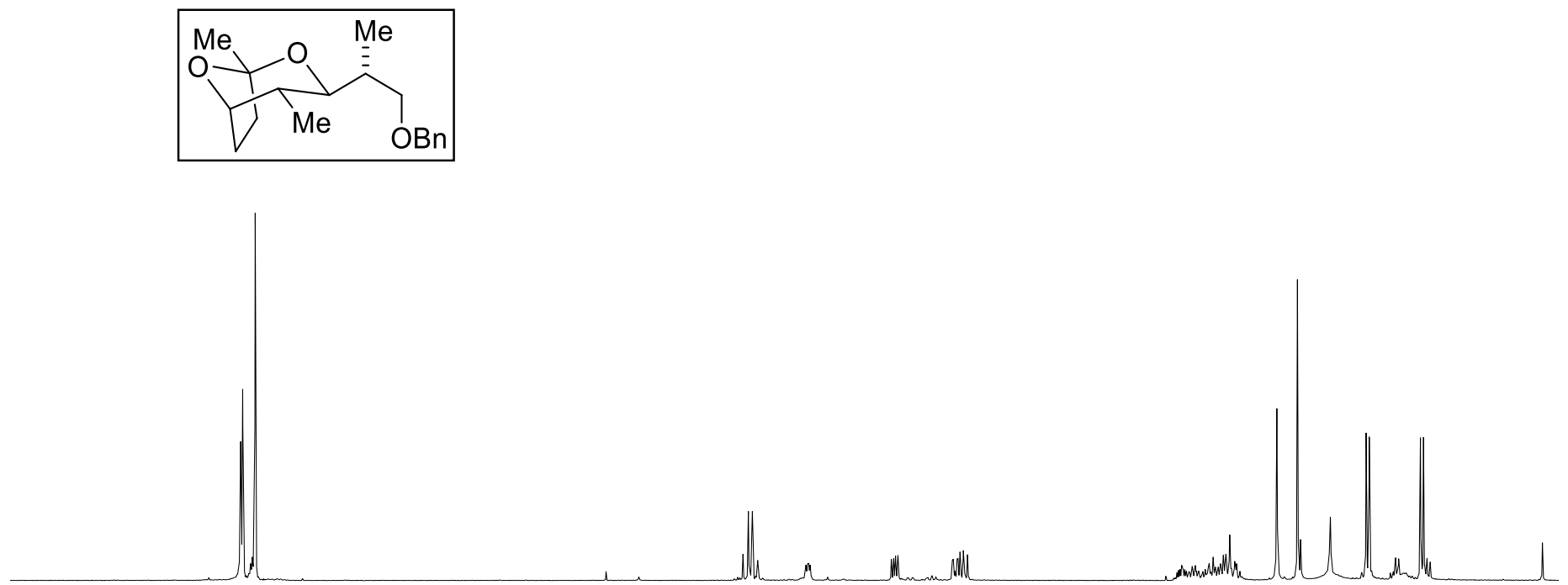

\begin{tabular}{llllllllllllllllll}
\hline 8.5 & 8.0 & 7.5 & 7.0 & 6.5 & 6.0 & 5.5 & $\begin{array}{l}5.0 \\
\mathrm{f} 1(\mathrm{ppm})\end{array}$ & 4.5 & 4.0 & 3.5 & 3.0 & 2.5 & 2.0 & 1.5 & 1.0 & 0.5 & 0.
\end{tabular}

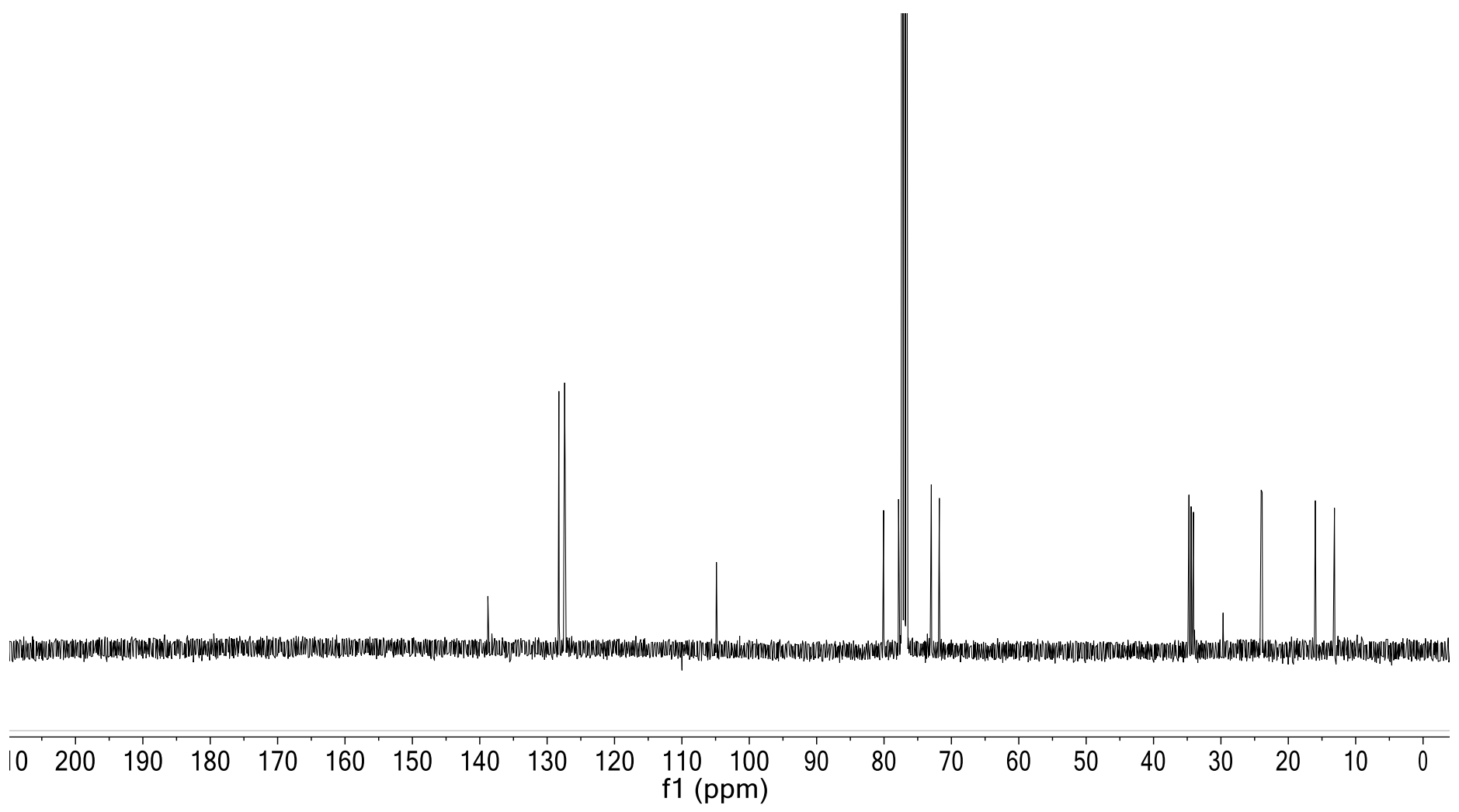




\section{$\mathrm{H}$ and C-NMR $\left(\mathrm{CDCl}_{3}\right)$ of compound 34}
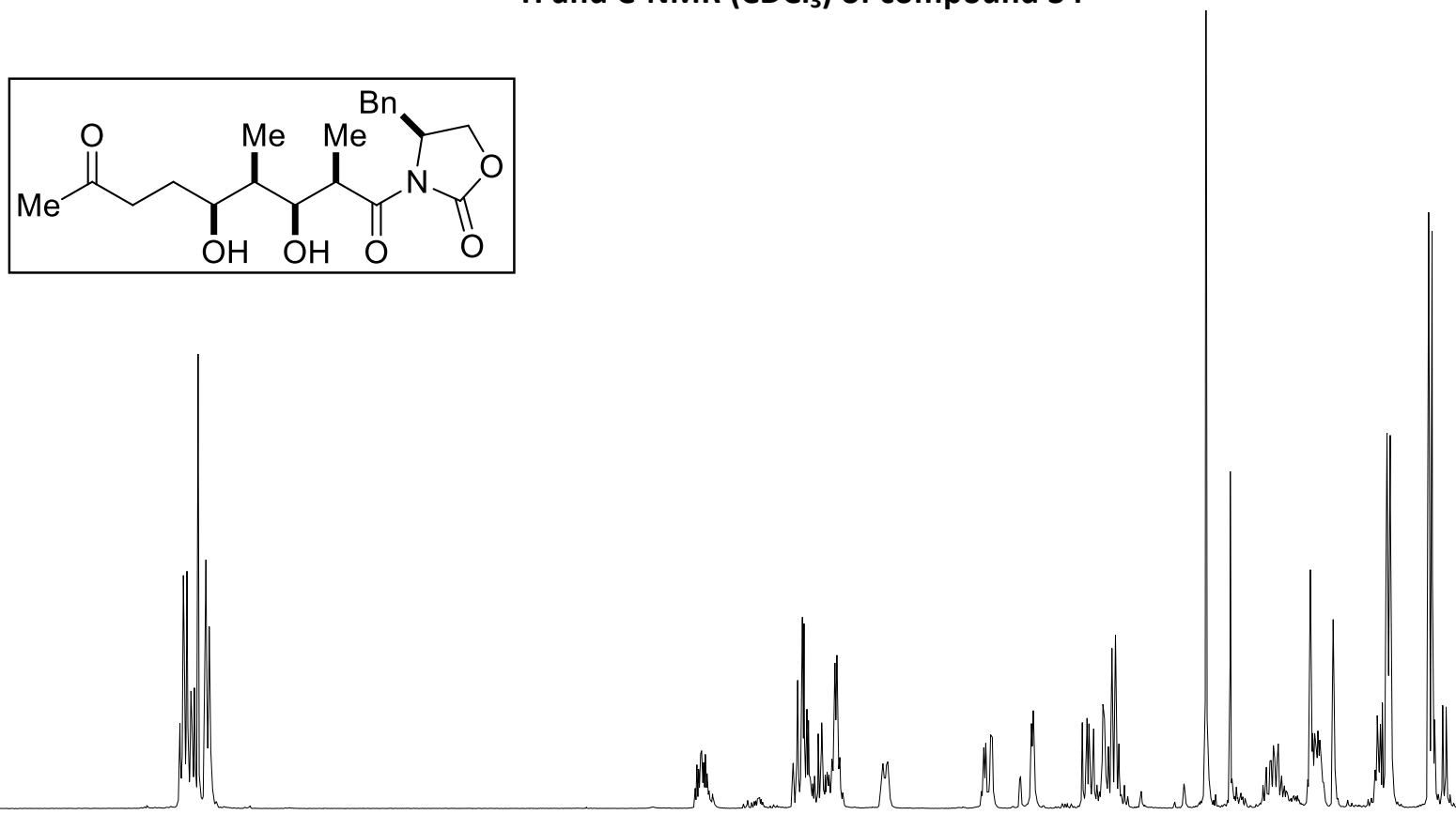

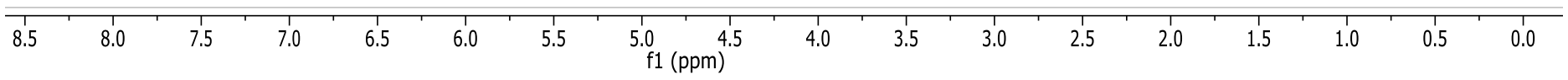
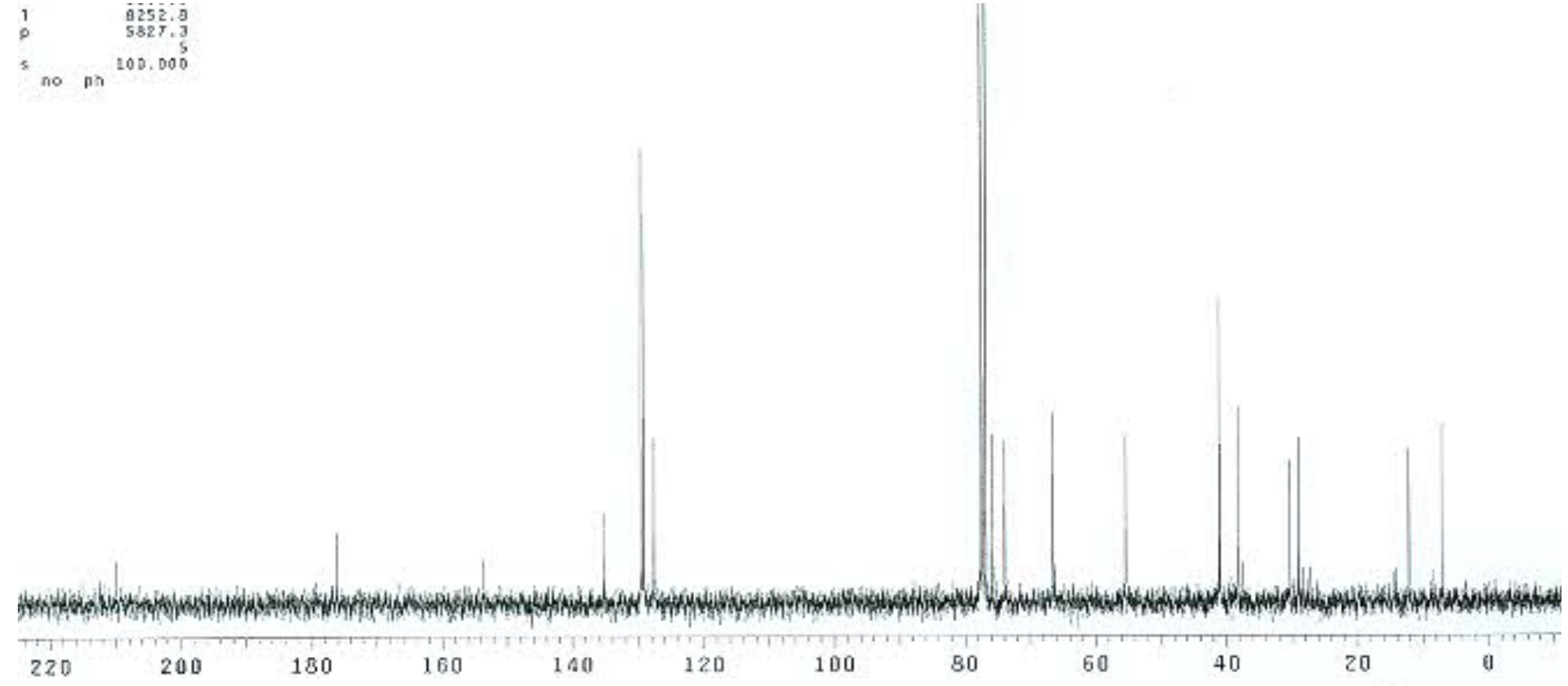


\section{$\mathrm{H}$ and C-NMR $\left(\mathrm{CDCl}_{3}\right)$ of compound 35}
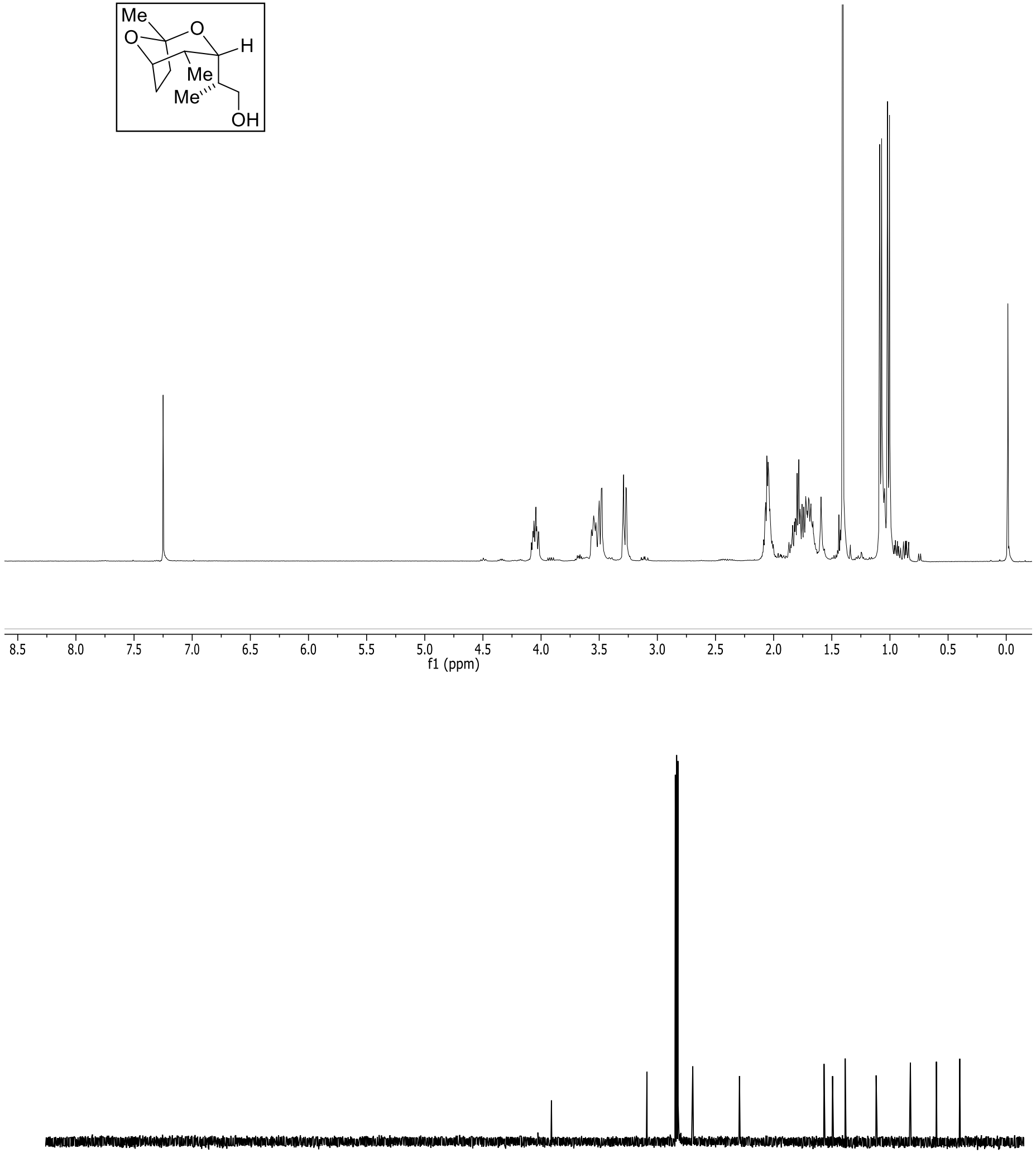

\begin{tabular}{|c|c|c|c|c|c|c|c|c|c|c|c|c|c|c|c|c|c|c|c|}
\hline $220 \quad 210$ & 200 & 190 & 180 & 170 & 160 & 150 & 140 & 130 & $\begin{array}{l}120 \underset{\text { f1 (ppm) }}{110} 100 \\
S-63\end{array}$ & 90 & 80 & 70 & 60 & 50 & 40 & 30 & 20 & 10 & 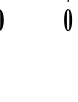 \\
\hline
\end{tabular}




\section{$\mathrm{H}$ and C-NMR $\left(\mathrm{CDCl}_{3}\right)$ of S2}

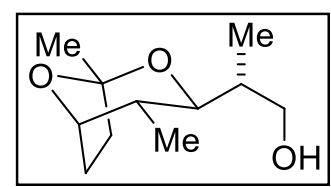

\begin{tabular}{|c|c|c|c|c|c|c|c|c|c|c|c|c|c|c|c|c|}
\hline 8.5 & 8.0 & 7.5 & 7.0 & 6.5 & 6.0 & 5.5 & $\begin{array}{c}5.0 \\
\mathrm{f} 1(\mathrm{ppm})\end{array}$ & 4.5 & 4.0 & 3.5 & 3.0 & 2.5 & 2.0 & 1.5 & 1.0 & 0.5 \\
\hline
\end{tabular}

\begin{tabular}{|c|c|}
\hline & $250^{\circ}$ \\
\hline in & 4.41 \\
\hline 1 & 3251. \\
\hline I & 5927. \\
\hline & 100.0000 \\
\hline
\end{tabular}

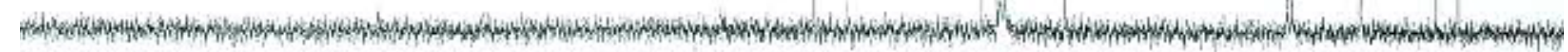

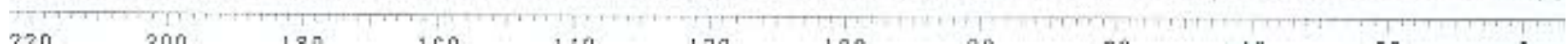




\section{$\mathrm{H}$ and C-NMR $\left(\mathrm{CDCl}_{3}\right)$ of 38}
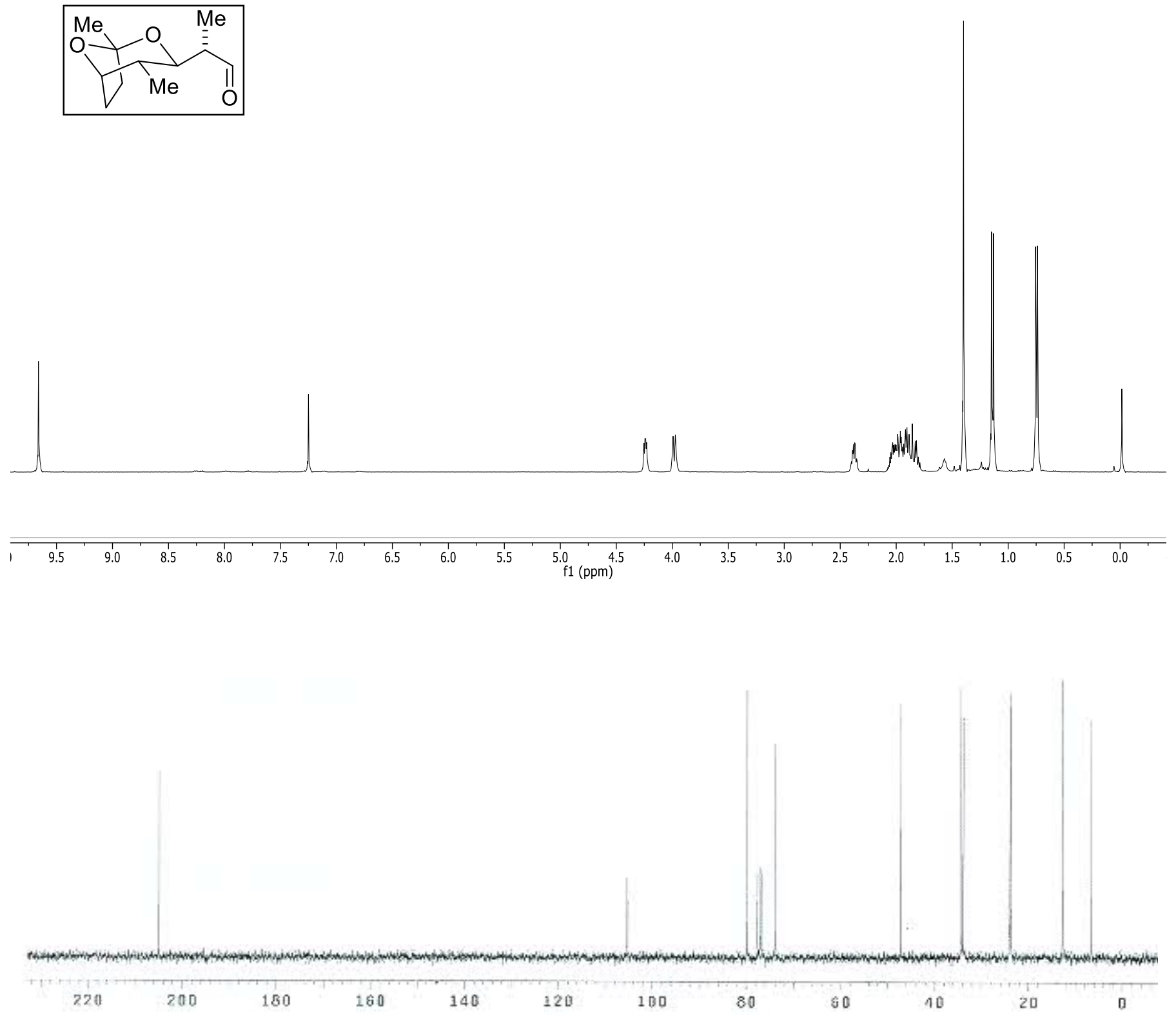


\section{$\mathrm{H}$ and C-NMR $\left(\mathrm{CDCl}_{3}\right)$ of metathesis precursor S3}
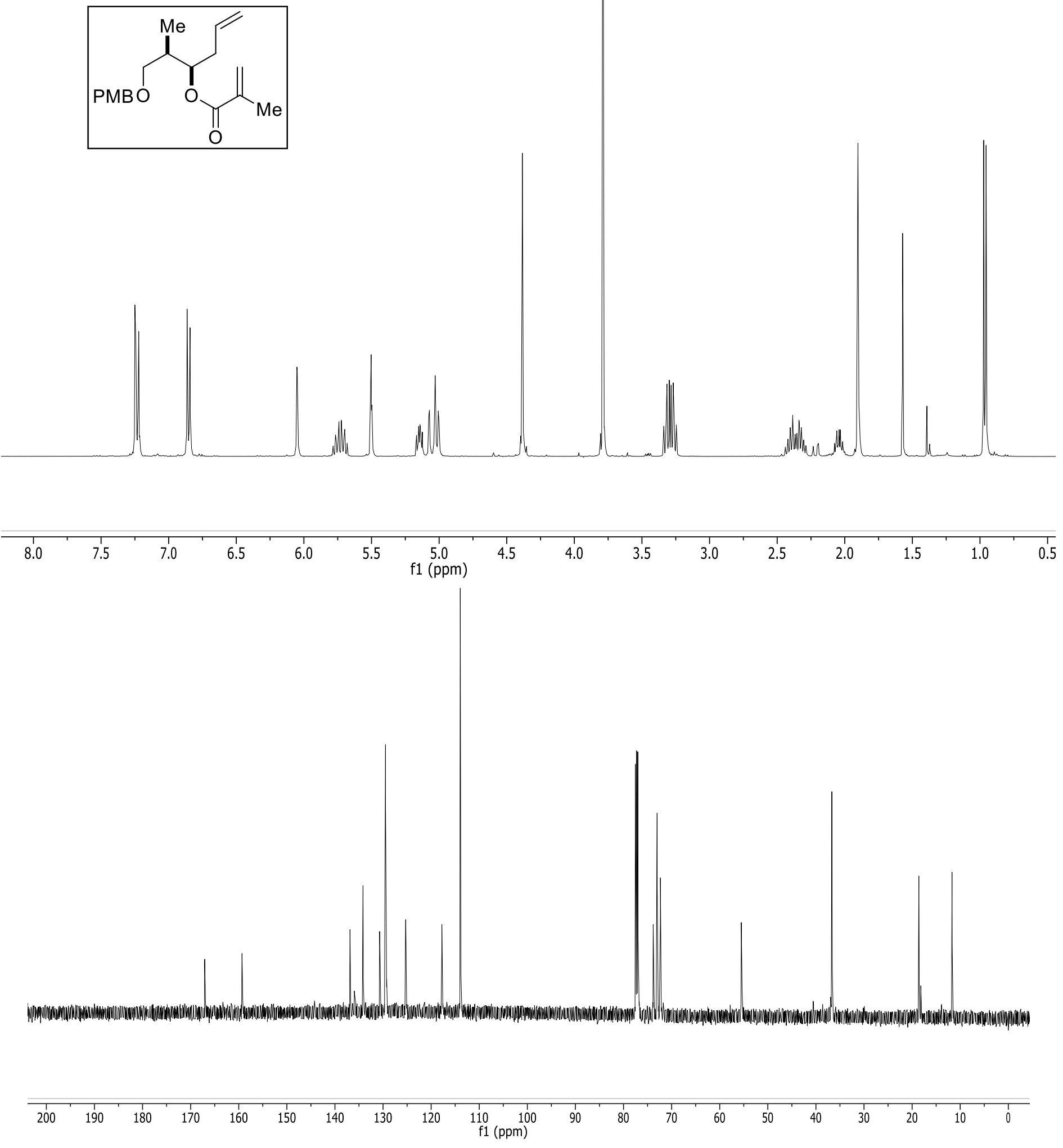


\section{$\mathrm{H}$ and C-NMR $\left(\mathrm{CDCl}_{3}\right)$ of compound $43 \mathrm{a}$}

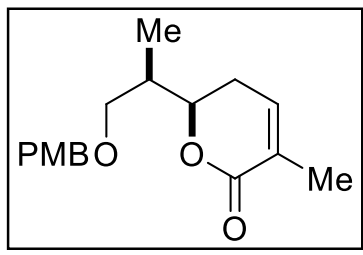

\begin{tabular}{|c|c|c|c|c|c|c|c|c|c|c|c|c|c|c|}
\hline 7.5 & 7.0 & 6.5 & 6.0 & 5.5 & $\begin{array}{c}5.0 \\
\mathrm{f} 1(\mathrm{ppm})\end{array}$ & 4.5 & 4.0 & 3.5 & 3.0 & 2.5 & 2.0 & 1.5 & 1.0 & 0.5 \\
\hline
\end{tabular}

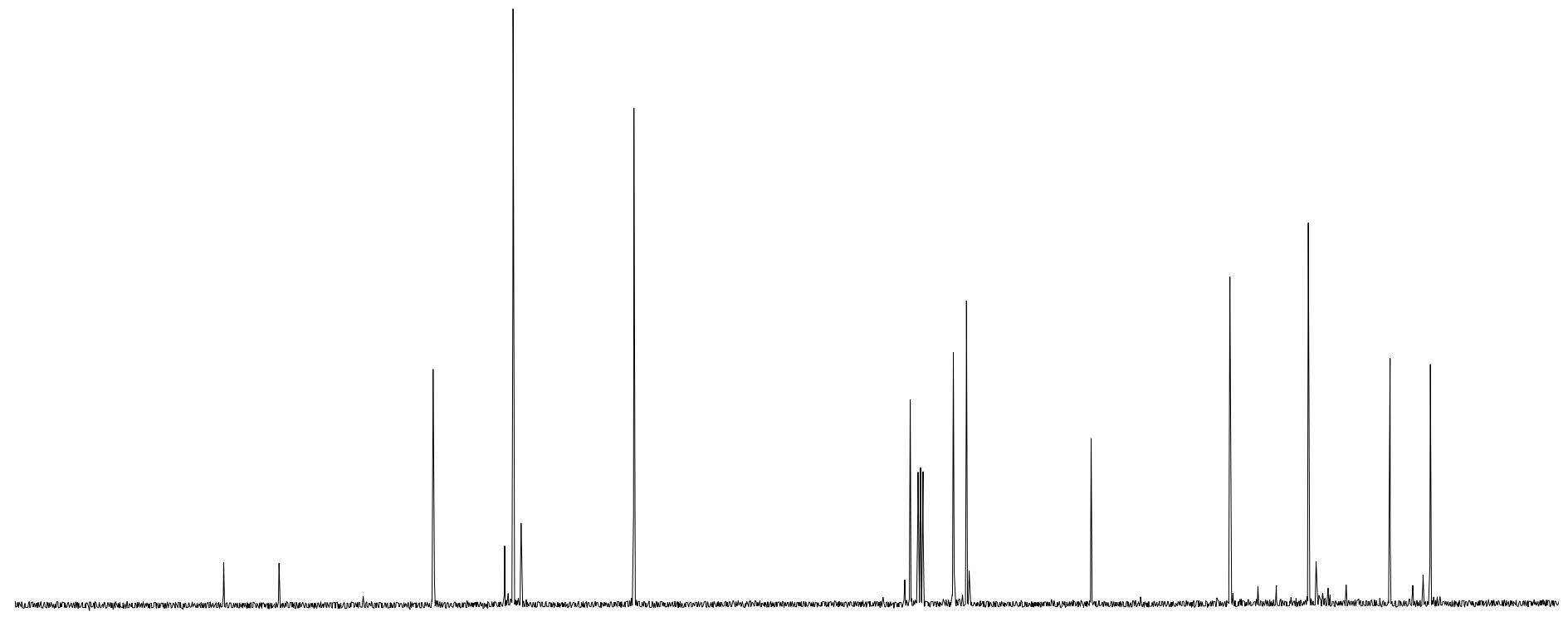

\begin{tabular}{|c|c|c|c|c|c|c|c|c|c|c|c|c|c|c|c|c|c|}
\hline 190 & $\begin{array}{c}1 \\
180\end{array}$ & 170 & 160 & $\stackrel{1}{150}$ & & $\begin{array}{l}1 \\
130\end{array}$ & 120 & $110 \quad 100$ & 90 & $\begin{array}{l}1 \\
80\end{array}$ & 70 & 1 & $\begin{array}{l}1 \\
50\end{array}$ & 40 & 30 & & 10 \\
\hline
\end{tabular}




\section{$\mathrm{H}$ and C-NMR $\left(\mathrm{CDCl}_{3}\right)$ of alcohol S4}
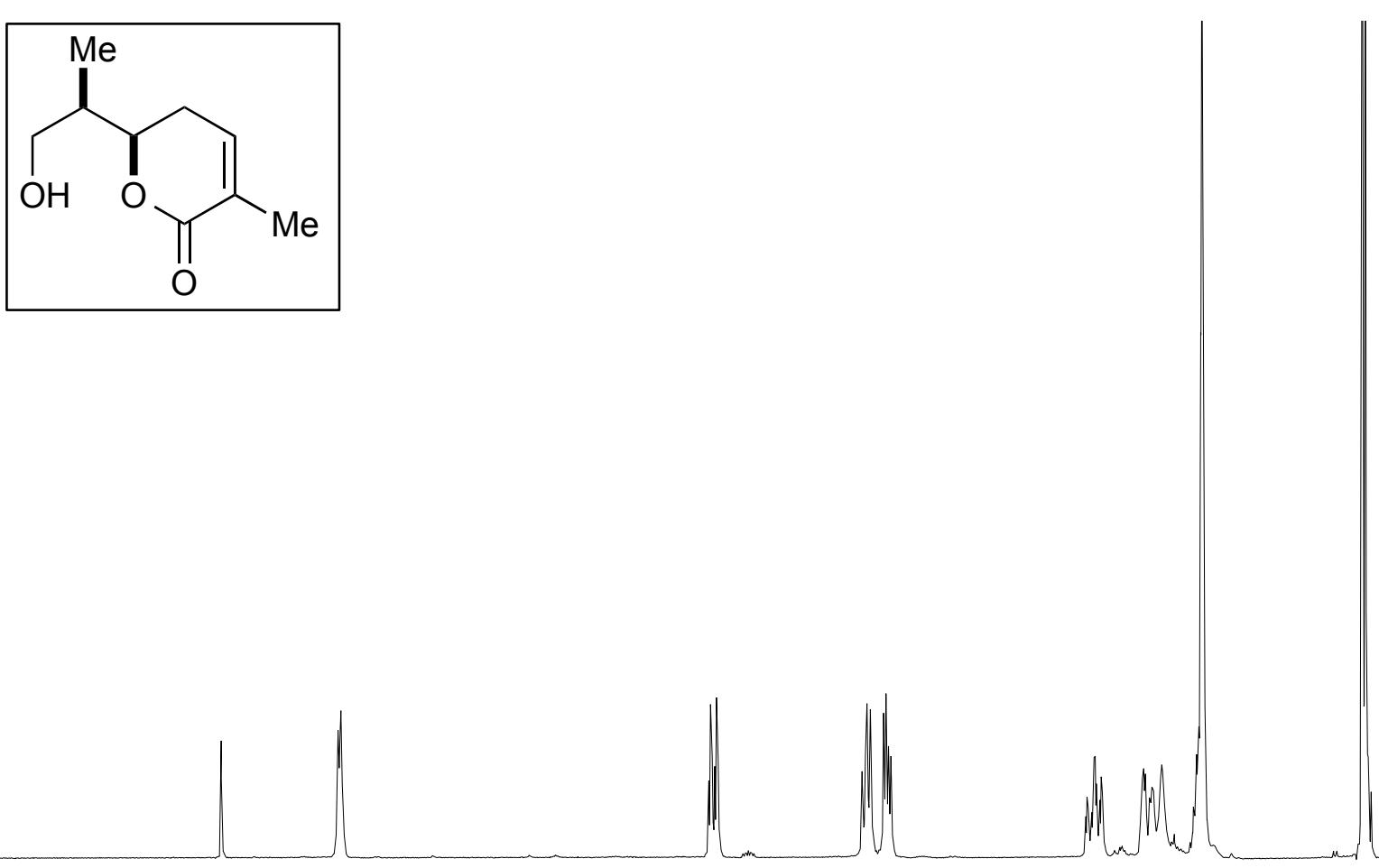

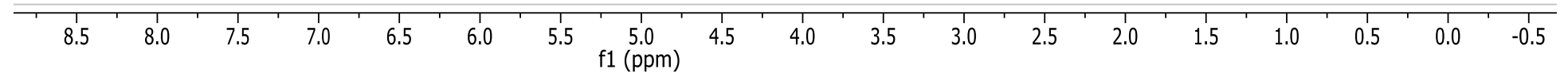

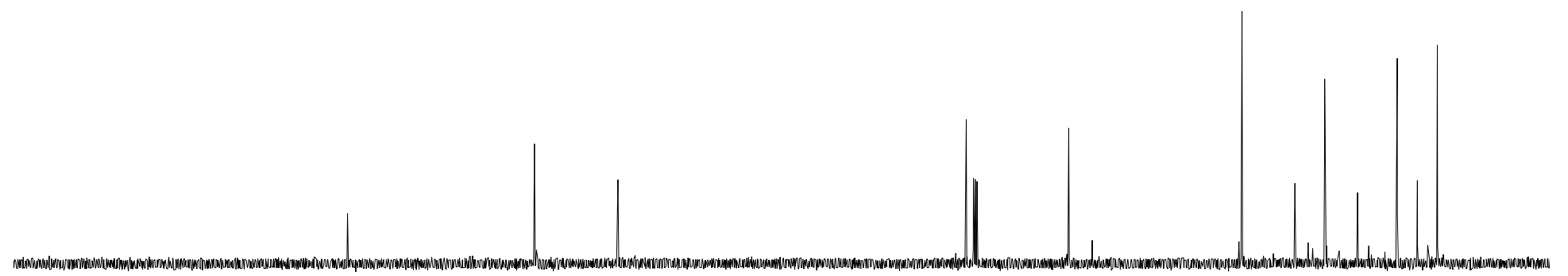

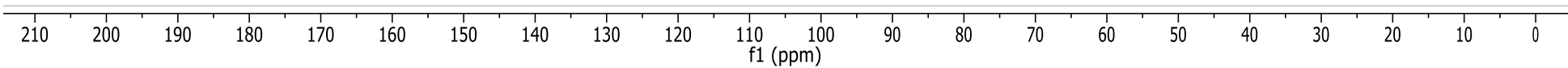




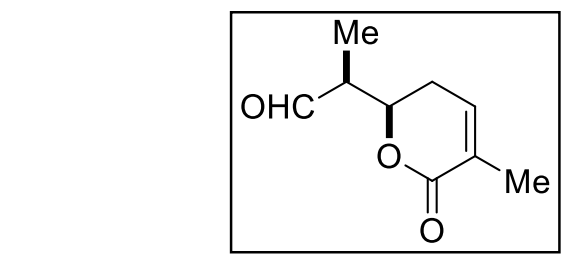

\section{$\mathrm{H}$ and C-NMR $\left(\mathrm{CDCl}_{3}\right)$ of compound 14a}

Hand C-NMR (CDCl $)$ of compound $14 a$
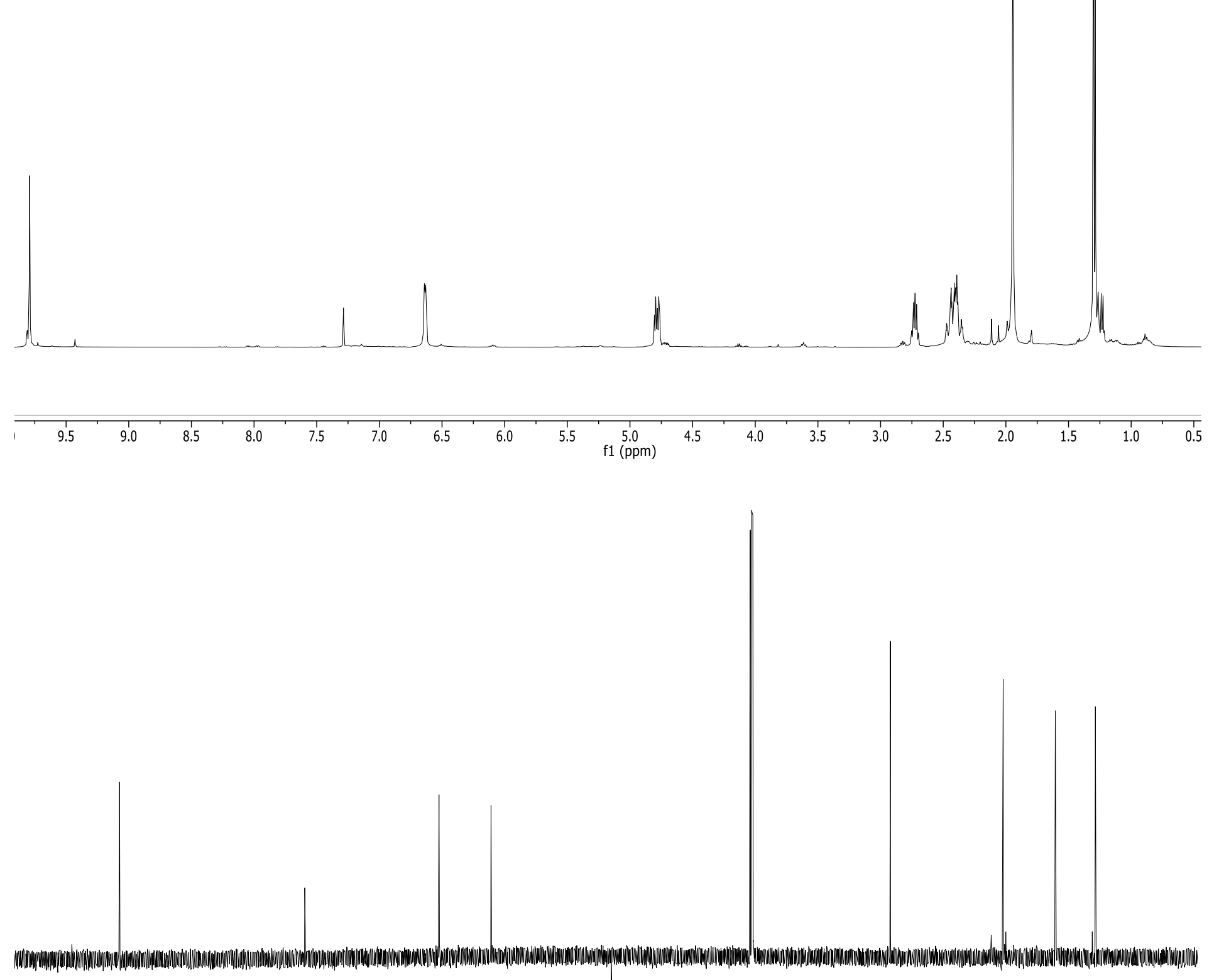

\begin{tabular}{|c|c|c|c|c|c|c|c|c|c|c|c|c|c|c|c|c|c|c|c|c|c|c|}
\hline 220 & 210 & 200 & 190 & 180 & 170 & 160 & 150 & 140 & 130 & 120 & $\begin{array}{c}110 \quad 100 \\
\text { f1 (ppm) }\end{array}$ & 90 & 80 & 70 & 60 & $\begin{array}{l}1 \\
50\end{array}$ & 40 & 30 & 20 & 10 & 1 & -11 \\
\hline
\end{tabular}




\section{$\mathrm{H}$ and C-NMR $\left(\mathrm{CDCl}_{3}\right)$ of compound $44 \mathrm{a}$}
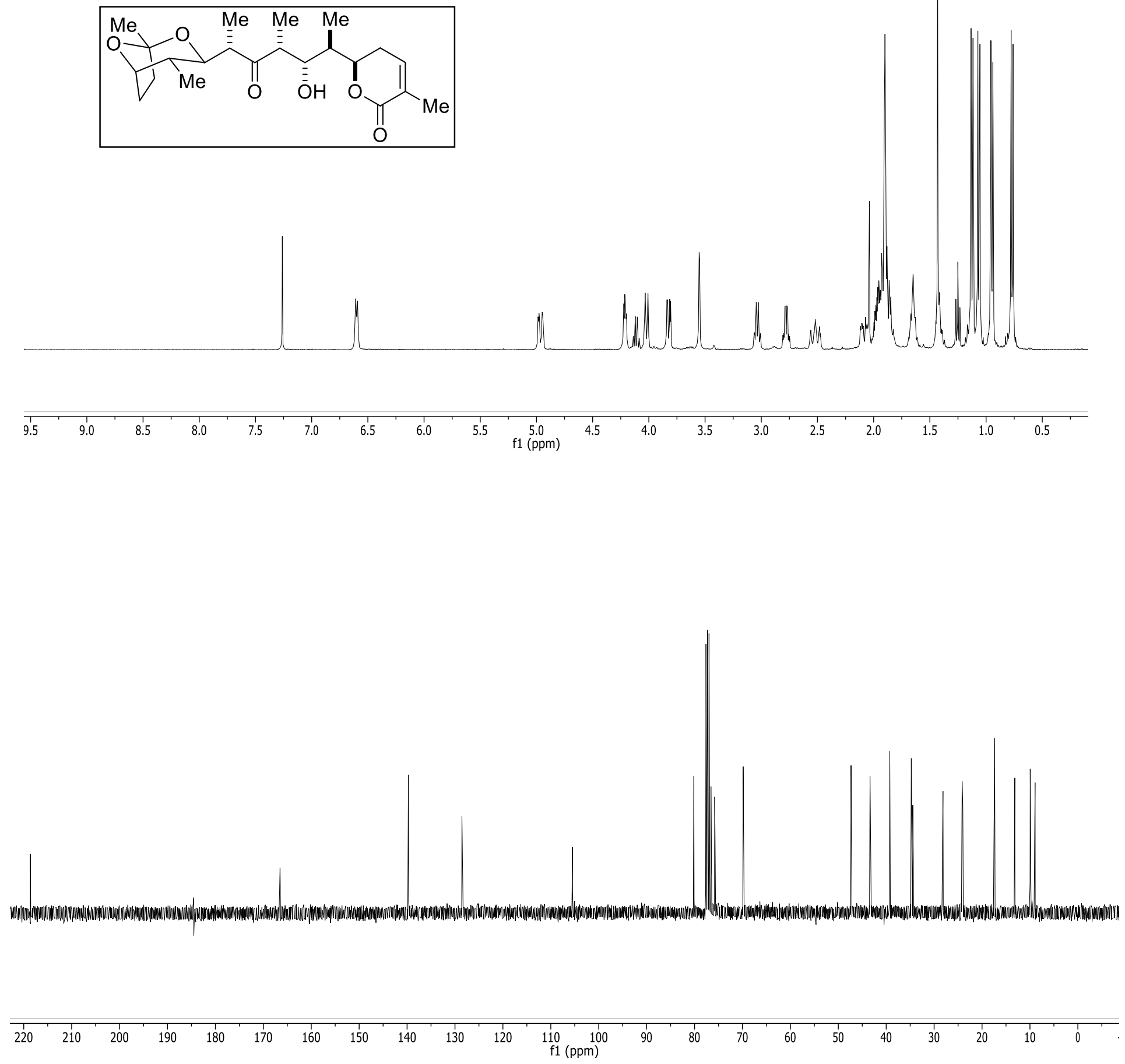


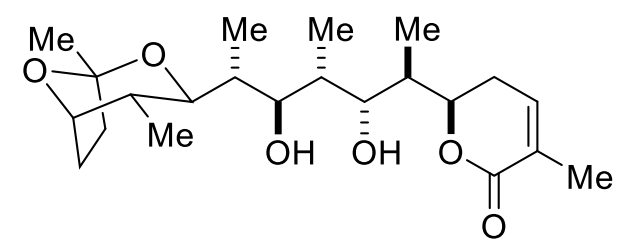

S5 (in $\mathrm{CDCl}_{3}, 600 \mathrm{MHz}$ )

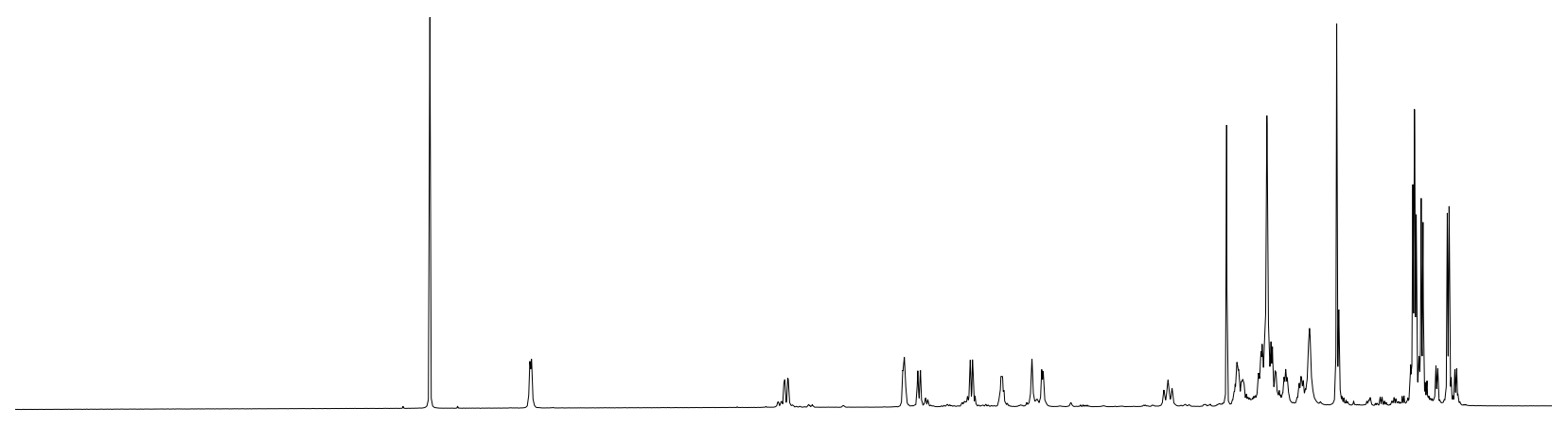

\begin{tabular}{|c|c|c|c|c|c|c|c|c|c|c|c|c|c|c|c|c|c|c|c|}
\hline 0 & 9.5 & 9.0 & 8.5 & 8.0 & 7.5 & 7.0 & 6.5 & 6.0 & 5.5 & $\begin{array}{c}5.0 \\
\mathrm{f} 1(\mathrm{ppm})\end{array}$ & 4.5 & 4.0 & 3.5 & 3.0 & 2.5 & 2.0 & 1.5 & 1.0 & 0.5 \\
\hline
\end{tabular}

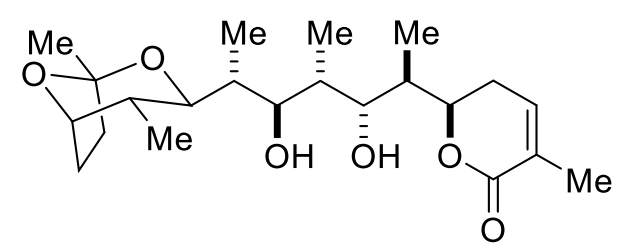

S5 (in $\mathrm{CDCl}_{3}, 600 \mathrm{MHz}$ )

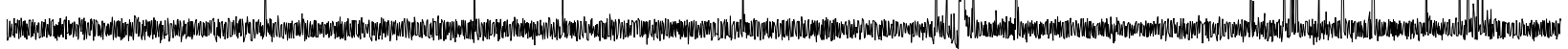

\begin{tabular}{llllllllllllllllll|l|l|l|l|l|l|l|l|l|l|l|l|l|l|l}
\hline 00 & 190 & 180 & 170 & 160 & 150 & 140 & 130 & 120 & 110 & $\begin{array}{c}100 \\
\mathrm{f} 1(\mathrm{ppm})\end{array}$ & 90 & 80 & 70 & 60 & 50 & 40 & 30 & 20 & 10 & 1
\end{tabular}


$\mathrm{H}\left(\mathrm{CD}_{3} \mathrm{OD}\right)$ and C-NMR $\left(\mathrm{CDCl}_{3}\right)$ of compound 45a
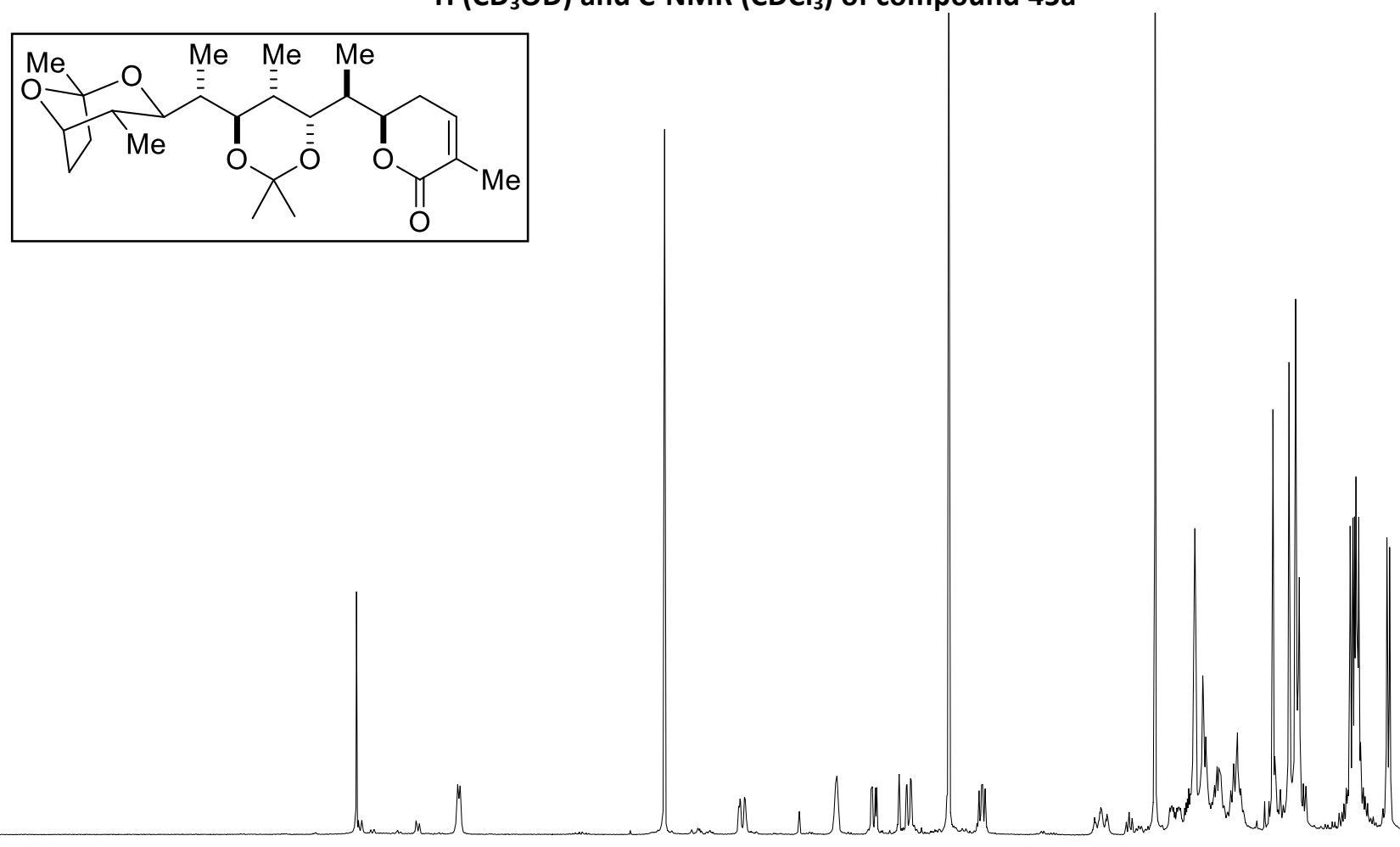

\begin{tabular}{llllllllllllllllllll}
\hline 9.5 & 9.0 & 8.5 & 8.0 & 7.5 & 7.0 & 6.5 & 6.0 & 5.5 & $\begin{array}{l}5.0 \\
\mathrm{f}^{\prime}(\mathrm{ppm})\end{array}$ & 4.5 & 4.0 & 3.5 & 3.0 & 2.5 & 2.0 & 1.5 & 1.0 & 0.5 & 0.0
\end{tabular}

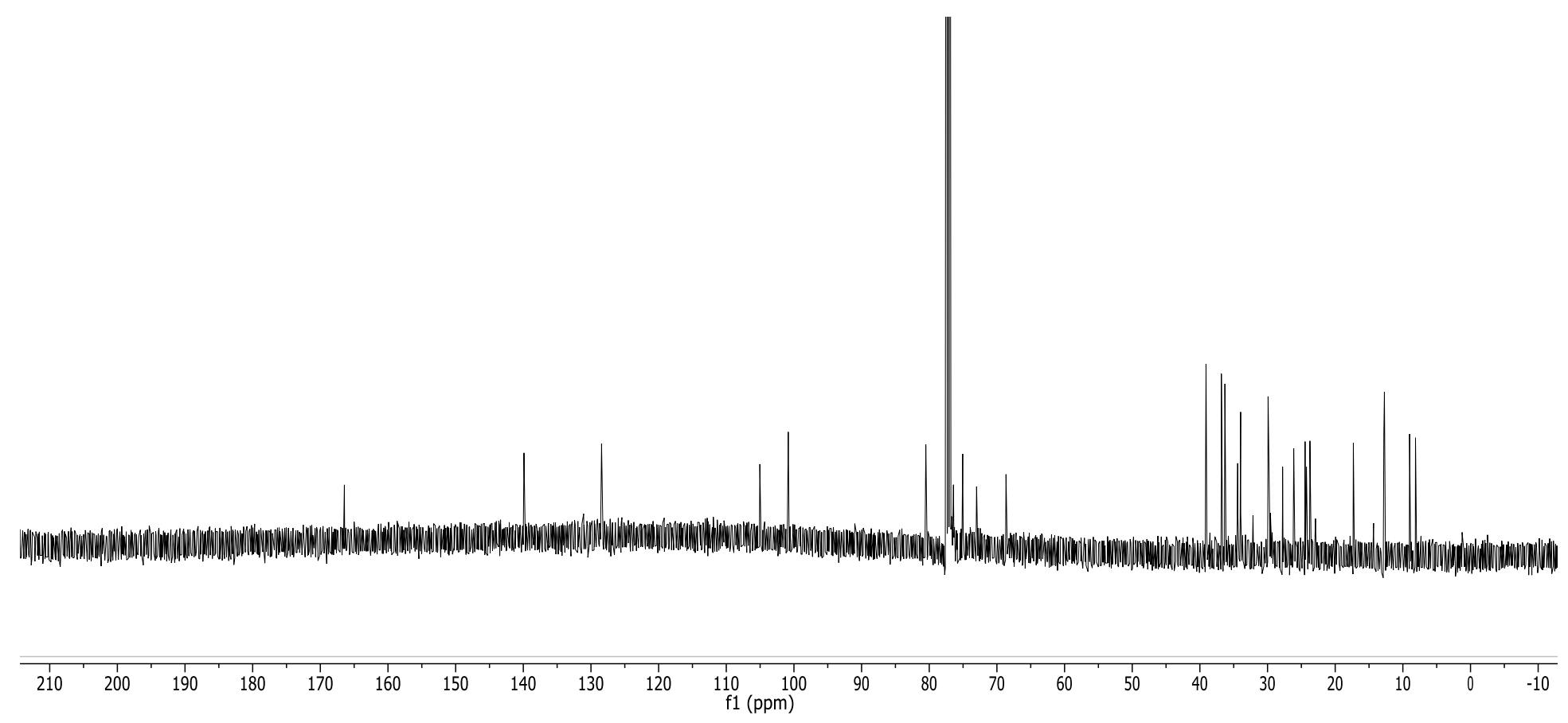


$\mathrm{H}$ and C-NMR $\left(\mathrm{CDCl}_{3}\right)$ of dienoic acid $\mathrm{S6}$

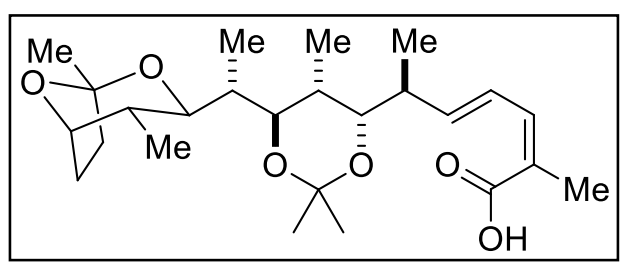

\begin{tabular}{|c|c|c|c|c|c|c|c|c|c|c|c|c|c|c|c|c|}
\hline 9.0 & 8.5 & 8.0 & 7.5 & 7.0 & 6.5 & 6.0 & 5.5 & $\begin{array}{lr}5.0 & 4.5 \\
\mathrm{f} 1(\mathrm{ppm})\end{array}$ & 4.0 & 3.5 & 3.0 & 2.5 & 2.0 & 1.5 & 1.0 & $0.5 \quad 0.0$ \\
\hline
\end{tabular}

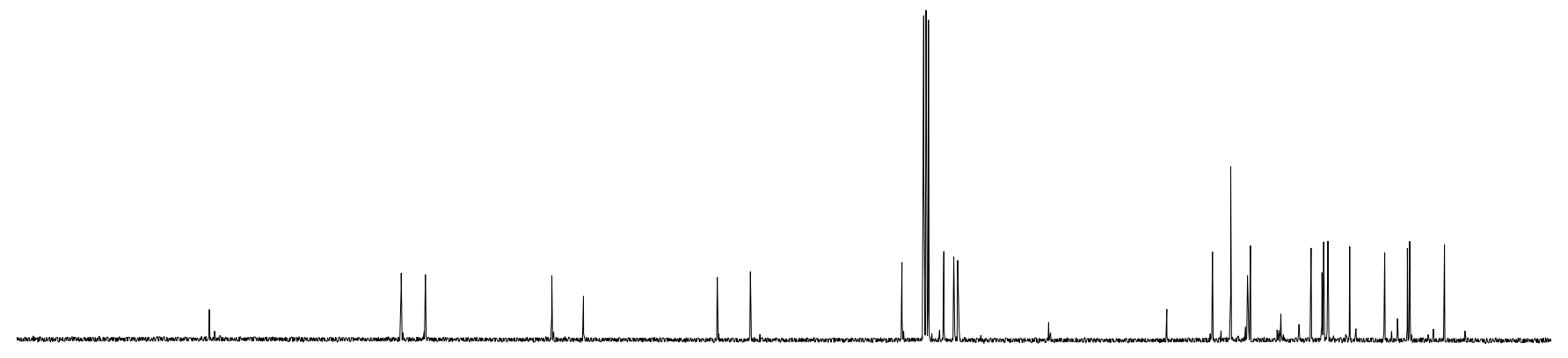

$\begin{array}{llllllllllllllllllll}190 & 180 & 170 & 160 & 150 & 140 & 130 & 120 & 110 & \begin{array}{l}100 \\ \mathrm{f} 1(\mathrm{ppm})\end{array} & 80 & 70 & 60 & 50 & 40 & 30 & 20 & 10 & 0\end{array}$




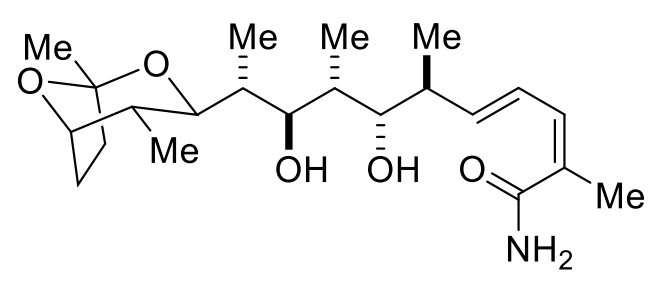

Saliniketal A (1a, in $\mathrm{CD}_{3} \mathrm{OD}, 400 \mathrm{MHz}$ )
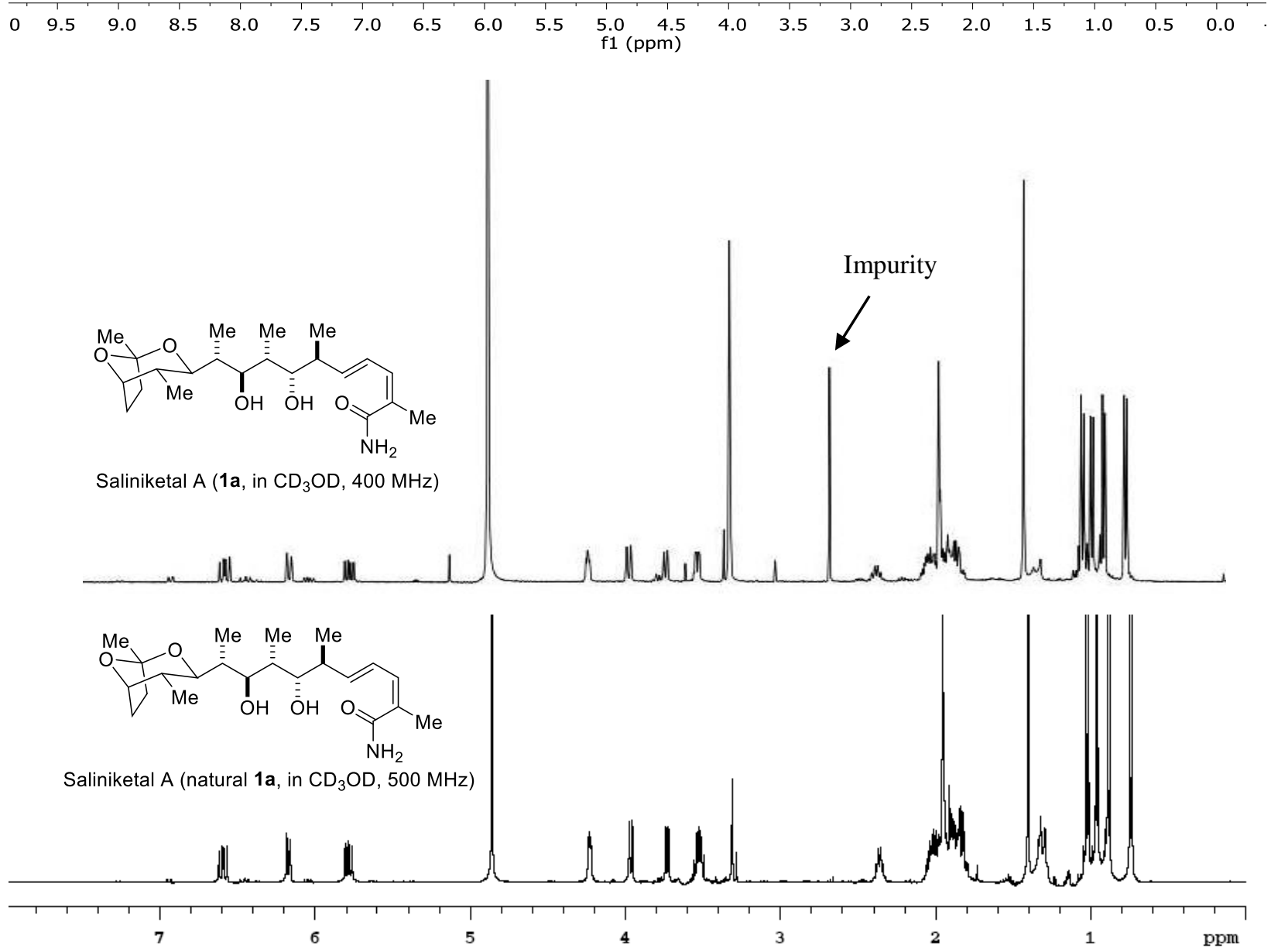


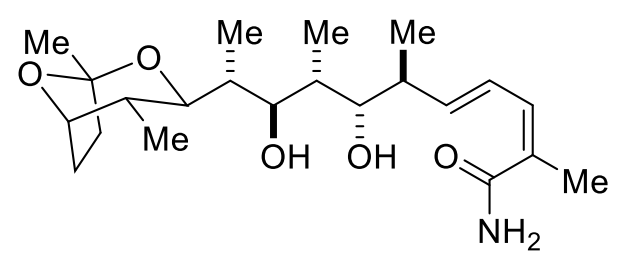

Saliniketal $\mathrm{A}\left(\mathbf{1} \mathbf{a}\right.$, in $\mathrm{CD}_{3} \mathrm{OD}, 100 \mathrm{MHz}$ )
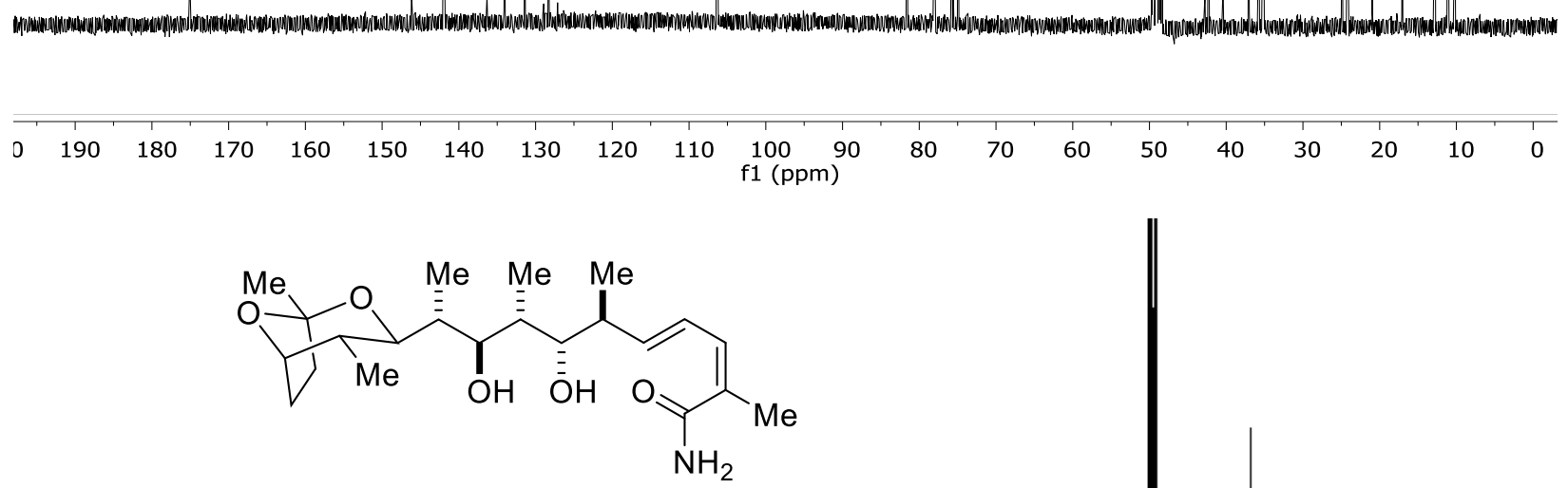

Saliniketal A (natural 1a, in $\mathrm{CD}_{3} \mathrm{OD}, 125 \mathrm{MHz}$ )

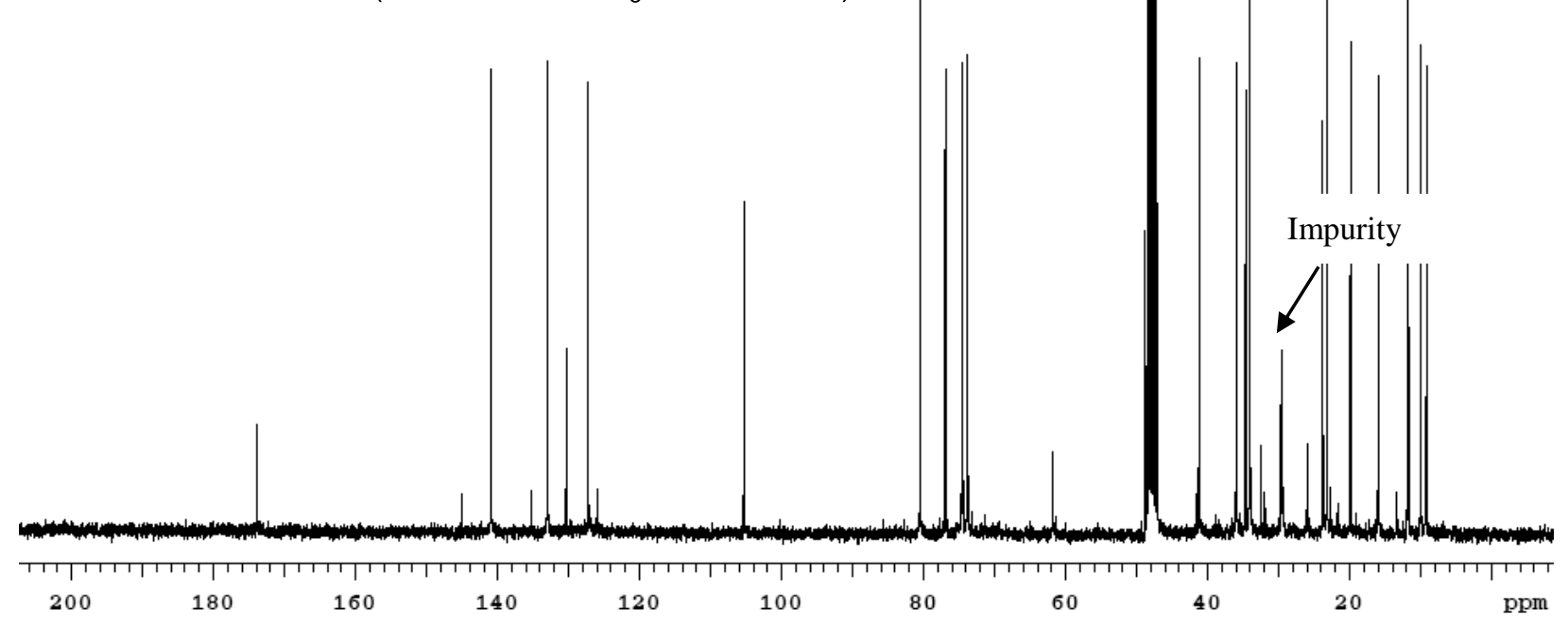




\section{$\mathrm{H}$ and $\mathrm{C}-\mathrm{NMR}\left(\mathrm{CDCl}_{3}\right)$ of compound 47}

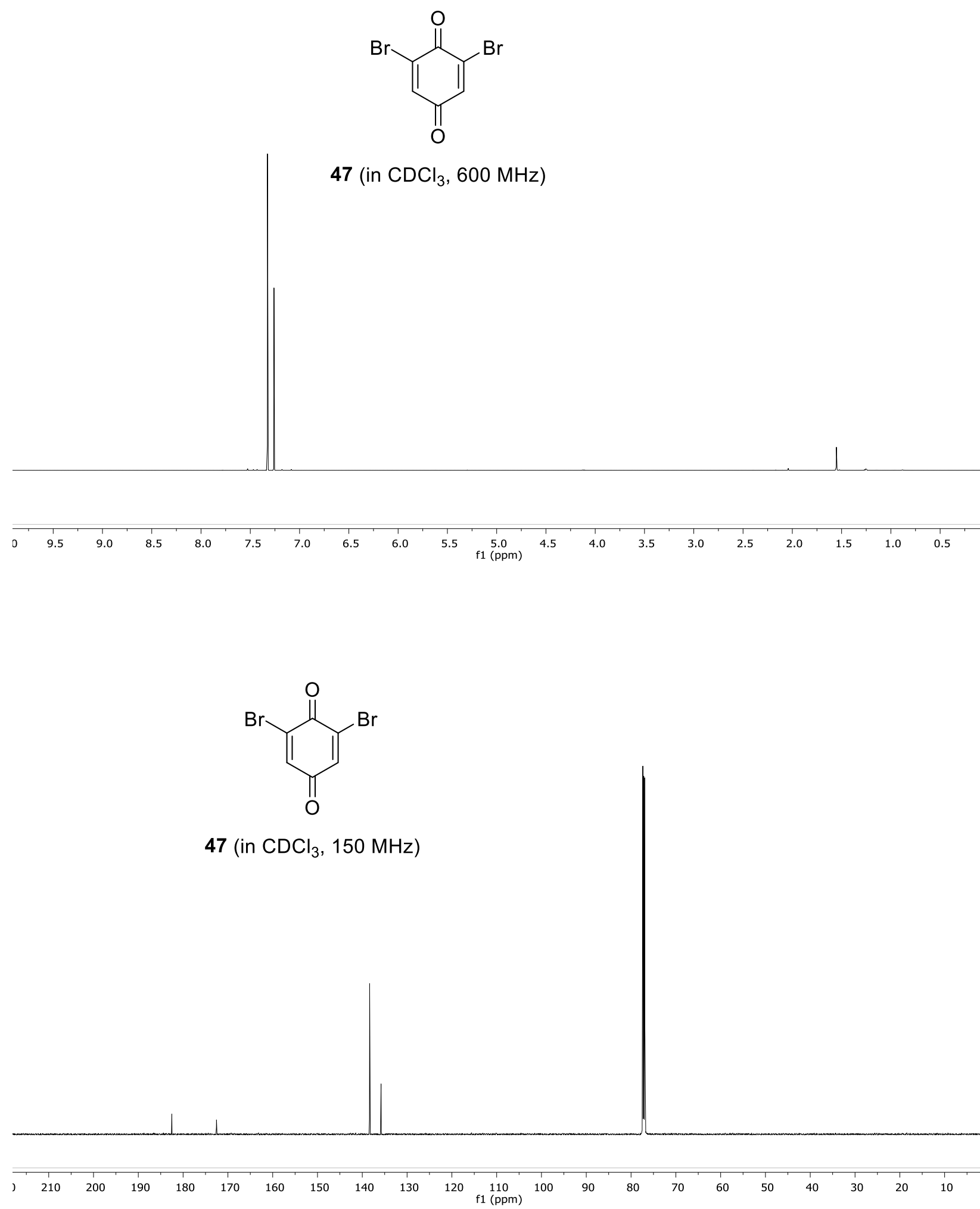




\section{$\mathrm{H}$ and C-NMR ( $\left.\mathrm{CDCl}_{3}\right)$ of compound S7}<smiles>CCOC(=O)C(C)=C(C)OC</smiles>

S7 (unstable crude in $\mathrm{CDCl}_{3}, 600 \mathrm{MHz}$ )

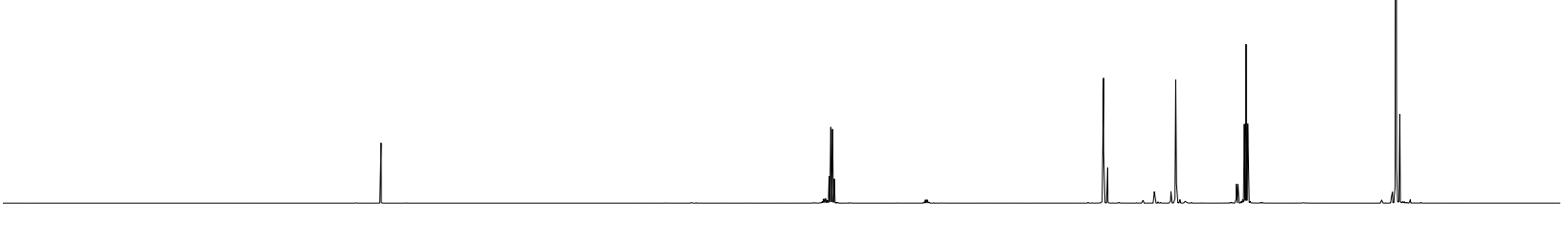

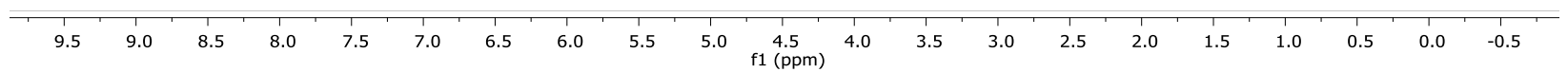

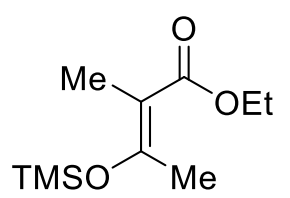

S7 (unstable crude in $\mathrm{CDCl}_{3}, 150 \mathrm{MHz}$ )

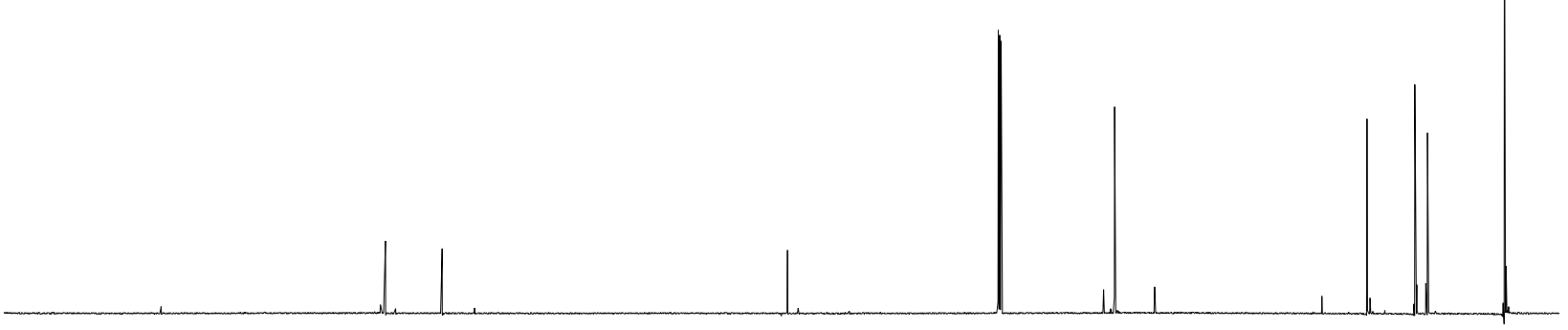

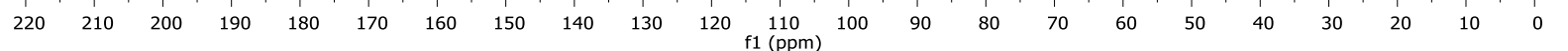




\section{$\mathrm{H}$ and C-NMR $\left(\mathrm{CD}_{3} \mathrm{OD}\right)$ of compound 53}<smiles>Cc1c(O)cc2c(c1O)C(=O)C(Br)=CC2=O</smiles>

$53\left(\mathrm{CD}_{3} \mathrm{OD}, 400 \mathrm{MHz}\right)$

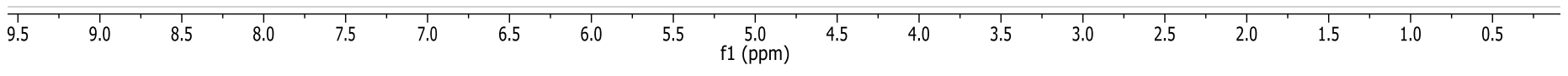<smiles>Cc1c(O)cc2c(c1O)C(=O)C(Br)=CC2=O</smiles>

$53\left(\mathrm{CD}_{3} \mathrm{OD}, 100 \mathrm{MHz}\right)$

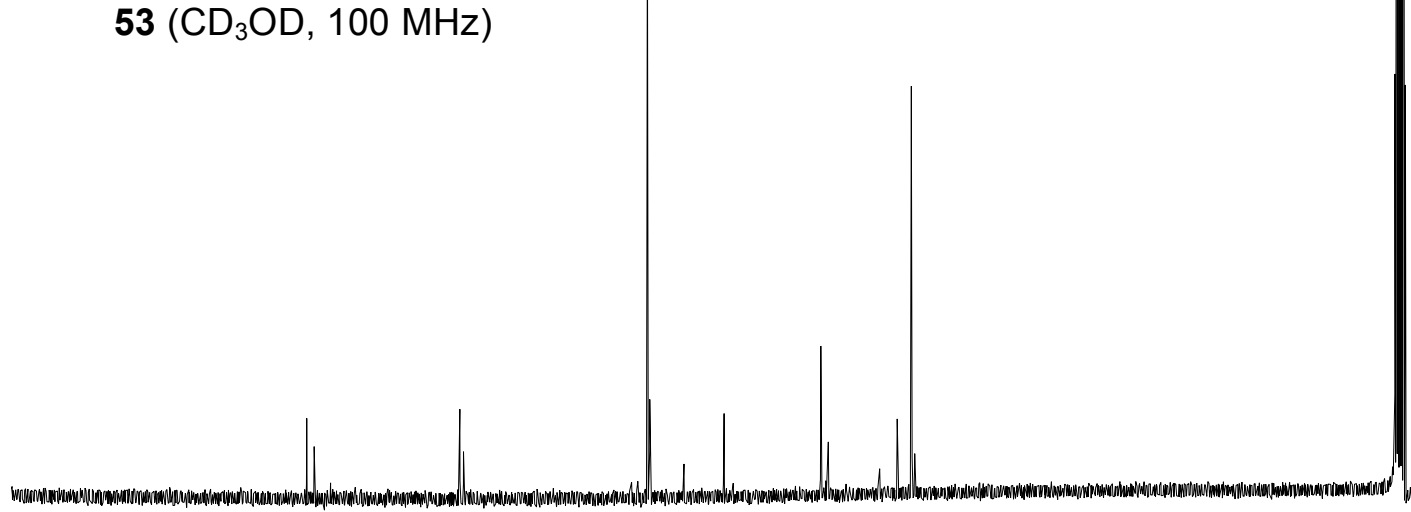

$\begin{array}{llllllllllll}) & 210 \quad 200 \quad 190 & 180 & 170 & 160 & 150 & 140 & 130 & 120 & 110 & 100\end{array}$ f1 (ppm) 


\section{$\mathrm{H}$ and C-NMR ( $\left.\mathrm{CDCl}_{3}\right)$ of compound 54}

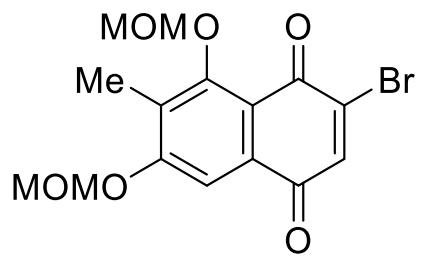

54 (in $\mathrm{CDCl}_{3}, 400 \mathrm{MHz}$ )

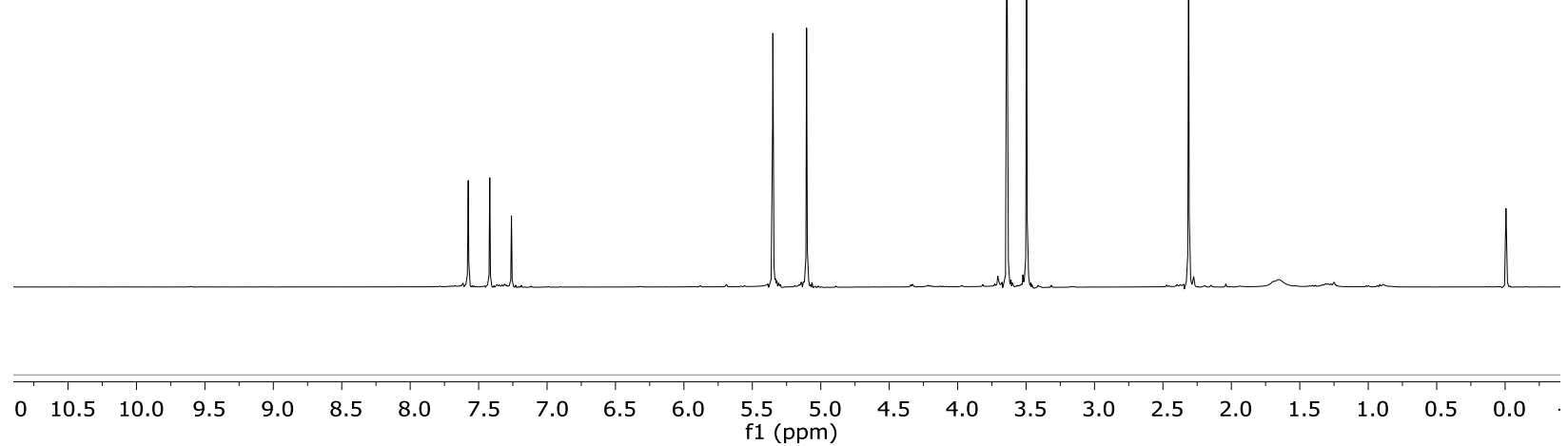<smiles>COc1cc2c(c(OC)c1OC)C(=O)C(Br)=CC2=O</smiles>

54 (in $\mathrm{CDCl}_{3}, 100 \mathrm{MHz}$ )

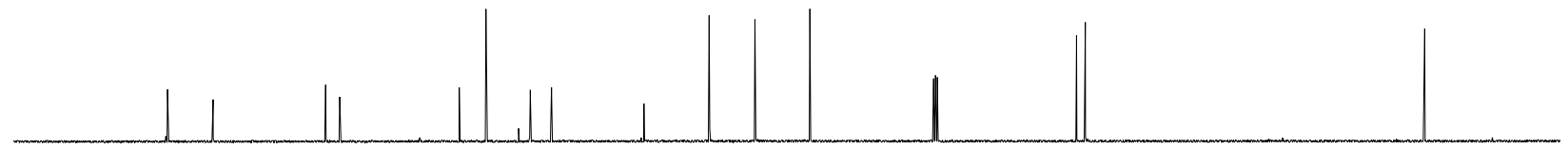

$\begin{array}{lllllllllllllllllllll}200 & 190 & 180 & 170 & 160 & 150 & 140 & 130 & 120 & 110 & \begin{array}{c}100 \\ f 1(\mathrm{ppm})\end{array} & 80 & 70 & 60 & 50 & 40 & 30 & 20 & 10 & 0\end{array}$




\section{H-NMR ( $\left.\mathrm{CDCl}_{3}\right)$ of compound S8}

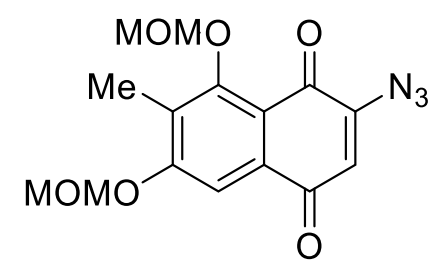

S8 (in $\mathrm{CDCl}_{3}, 400 \mathrm{MHz}$ )

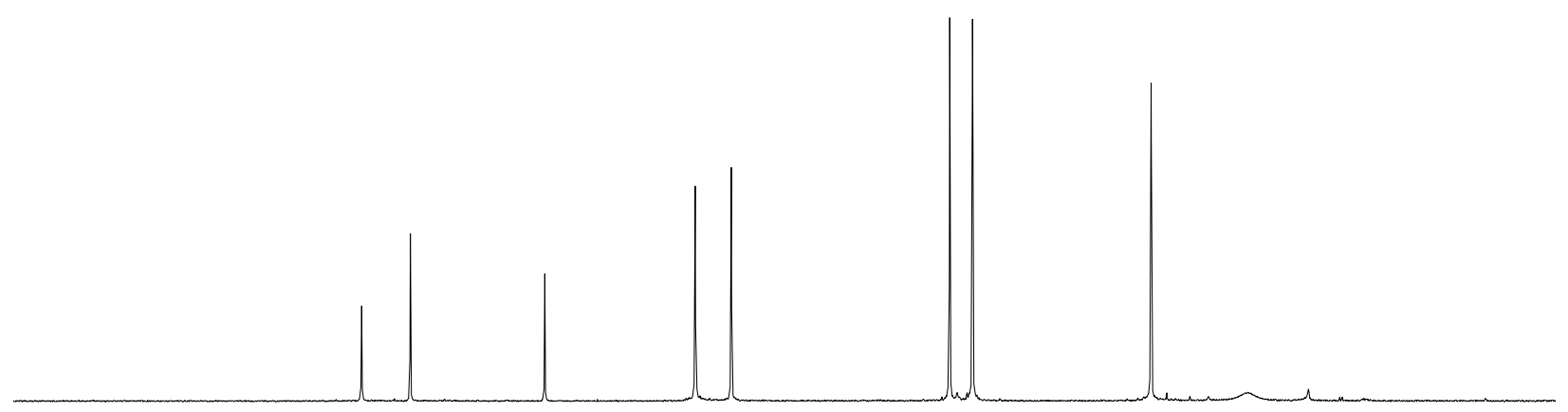

\begin{tabular}{lllll}
\hline 0 & 9.5 & 9.0 & 8.5 & 8.0
\end{tabular}
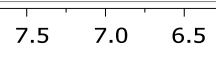

6.0 5.04 .5
f1 (ppm) 


\section{$\mathrm{H}$ and C-NMR ( $\left.\mathrm{CDCl}_{3}\right)$ of compound 55}<smiles>COc1cc2c(c(OC)c1OC)C(=O)C(N)=CC2=O</smiles>

55 (in $\mathrm{CDCl}_{3}, 400 \mathrm{MHz}$ )
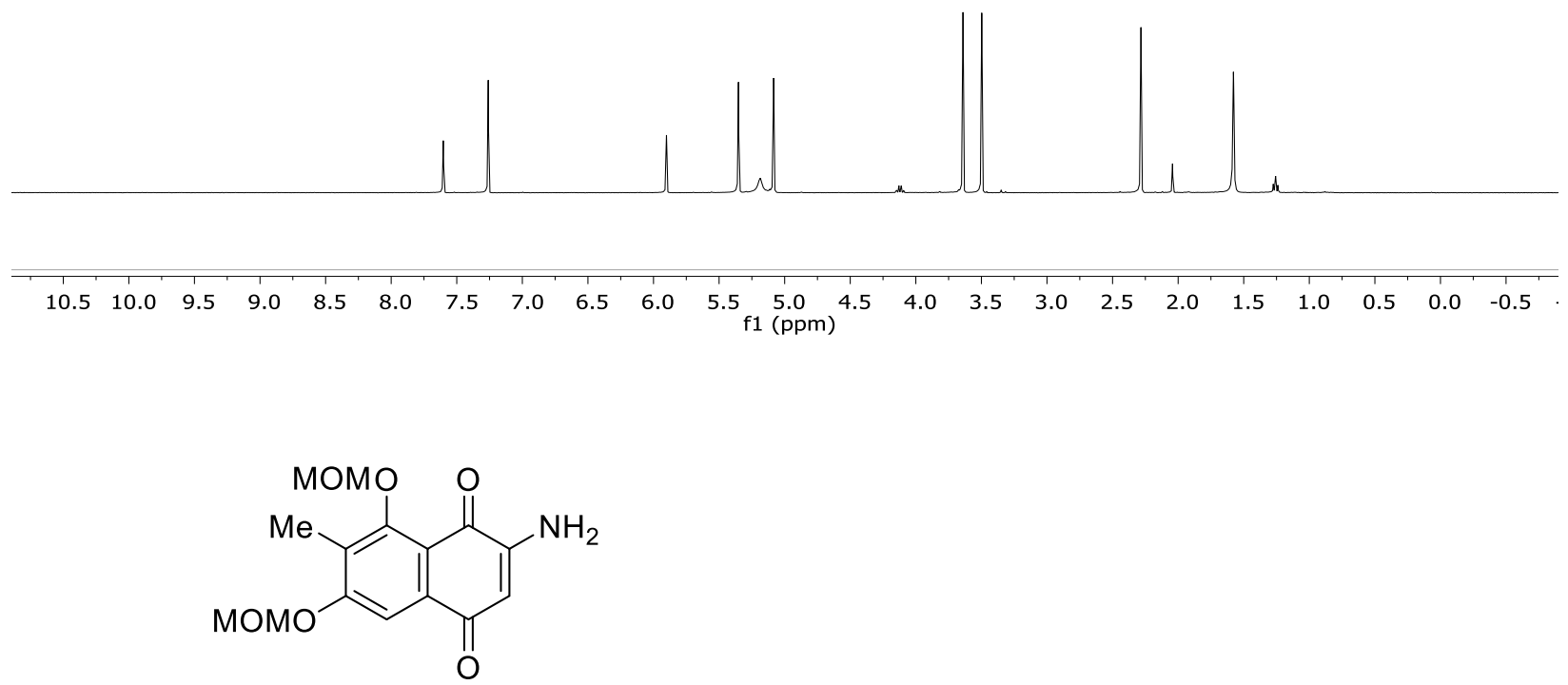

55 (in $\mathrm{CDCl}_{3}, 100 \mathrm{MHz}$ )

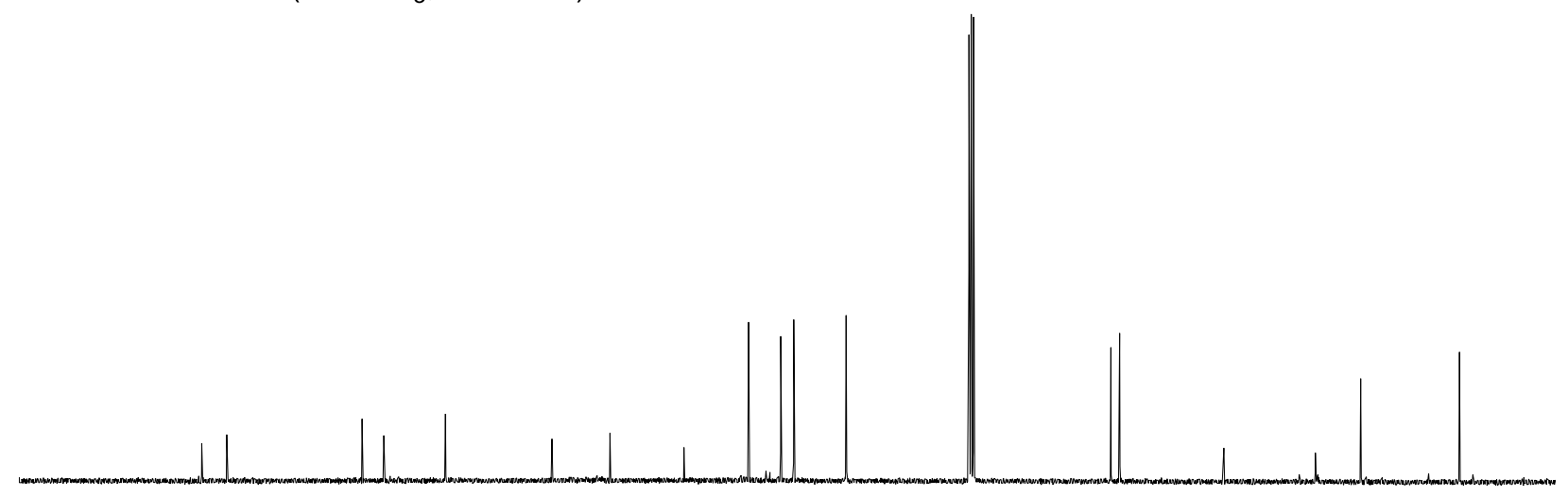

$\begin{array}{lllllllllll}200 & 190 & 180 & 170 & 160 & 150 & 140 & 130 & 120 & 110 & 100\end{array}$

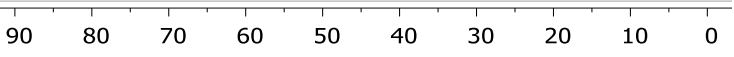




\section{$\mathrm{H}$ and C-NMR ( $\left.\mathrm{CDCl}_{3}\right)$ of compound 57}

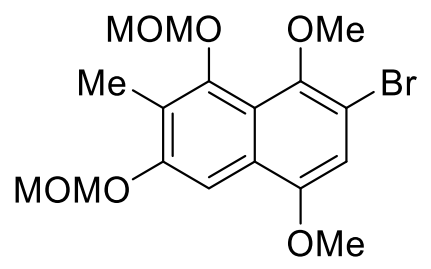

57 (in $\mathrm{CDCl}_{3}, 400 \mathrm{MHz}$ )

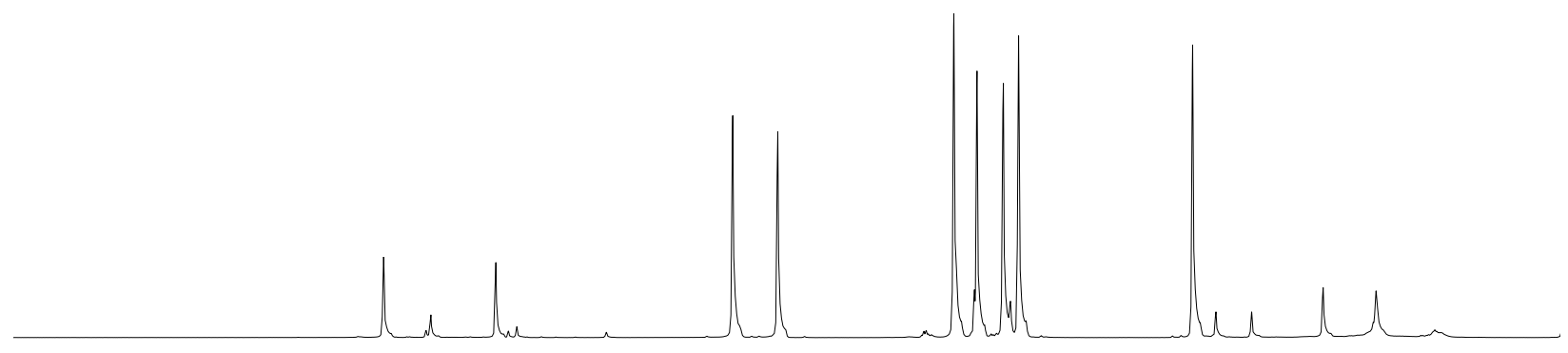

$\begin{array}{lllllllllllllllllll}0 & 9.5 & 9.0 & 8.5 & 8.0 & 7.5 & 7.0 & 6.5 & 6.0 & 5.5 \underset{\mathrm{f} 1}{\mathrm{~S}(\mathrm{ppm})} \mathbf{4 . 5} & 4.0 & 3.5 & 3.0 & 2.5 & 2.0 & 1.5 & 1.0 & 0.5\end{array}$

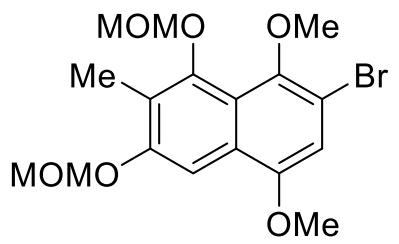

57 (in $\mathrm{CDCl}_{3}, 100 \mathrm{MHz}$ )

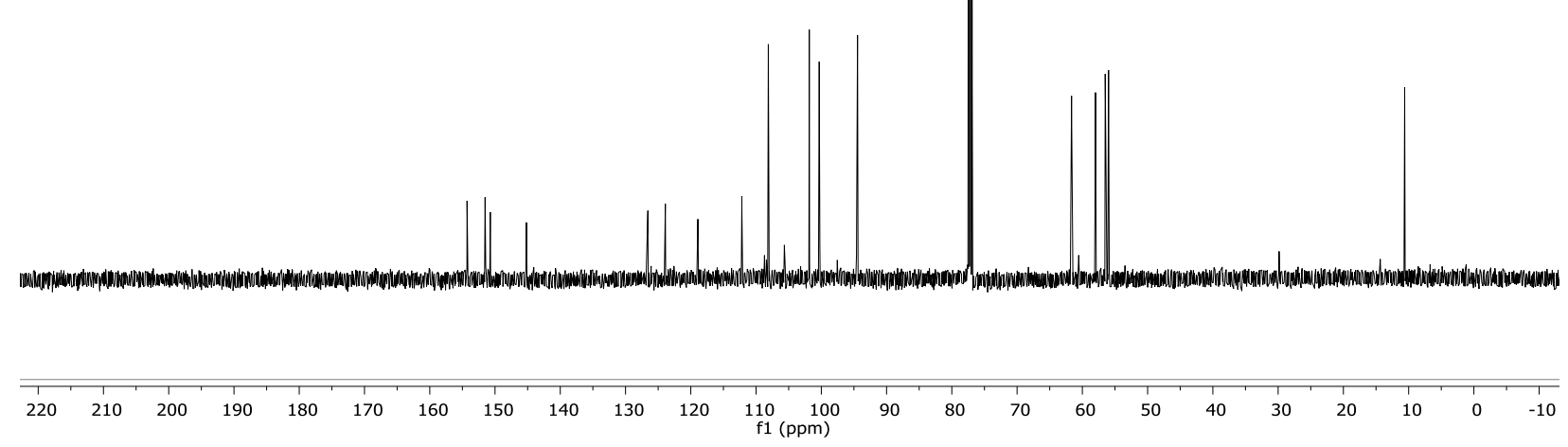




\section{$\mathrm{H}$ and C-NMR ( $\left.\mathrm{CDCl}_{3}\right)$ of compound 60}

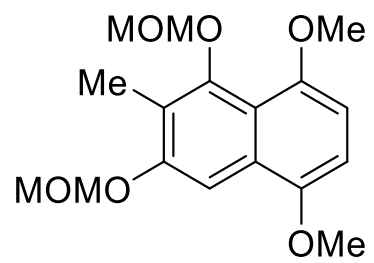

60 (in $\mathrm{CDCl}_{3}, 400 \mathrm{MHz}$ )
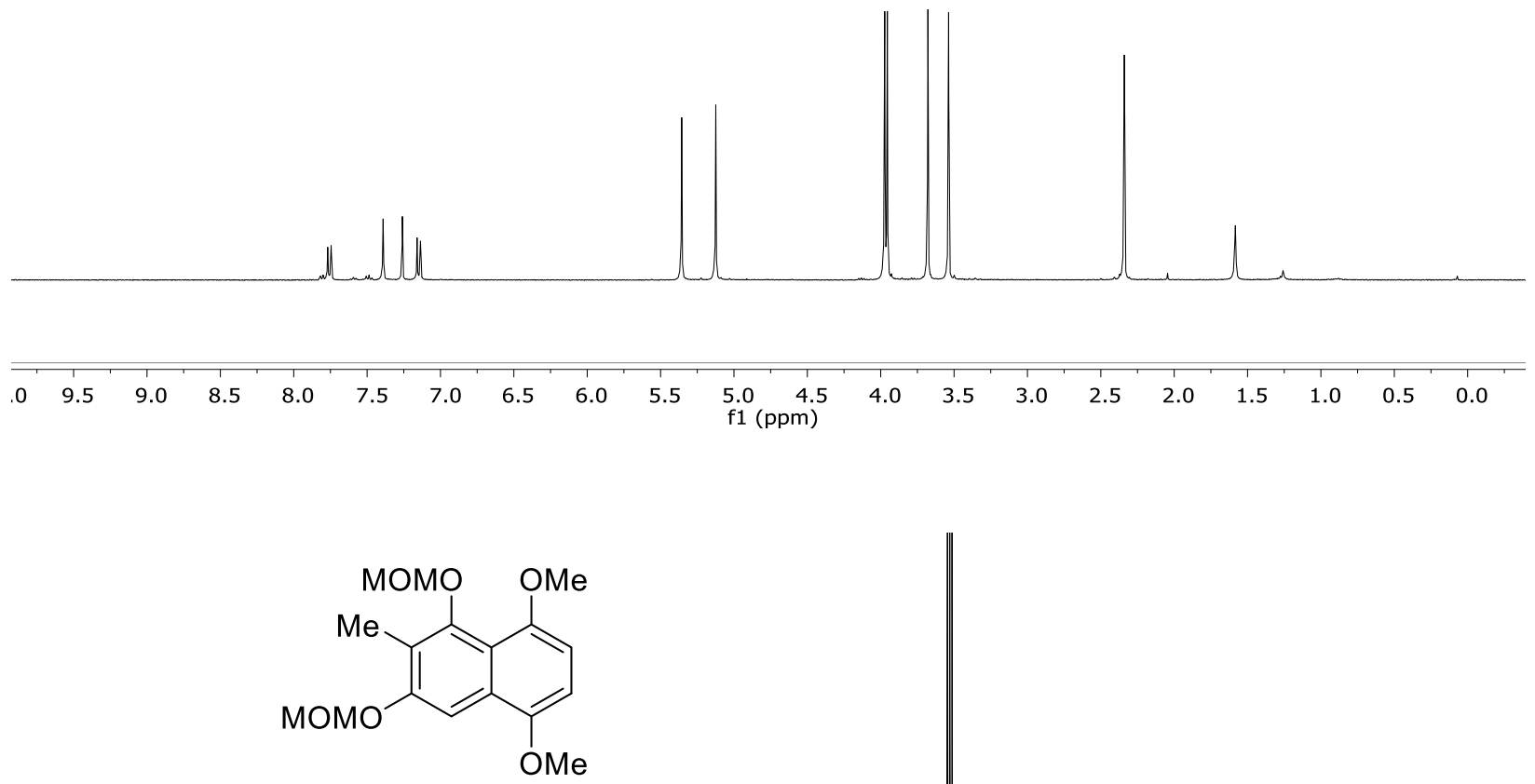

60 (in $\mathrm{CDCl}_{3}, 100 \mathrm{MHz}$ )
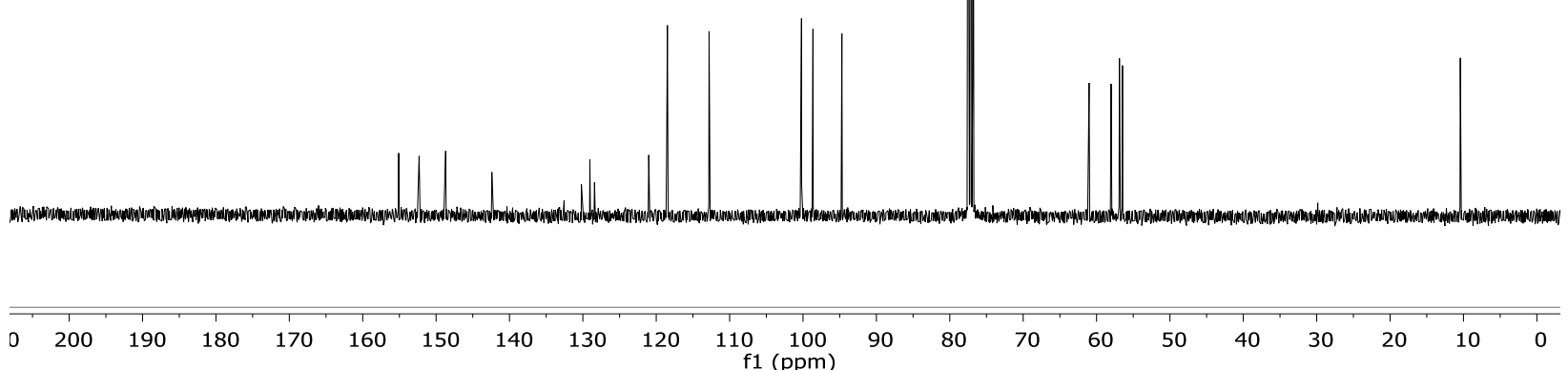


\section{$\mathrm{H}\left(\mathrm{CDCl}_{3}\right)$ and $\mathrm{C}\left(d_{6}-\mathrm{DMSO}\right)$-NMR of compound 61}

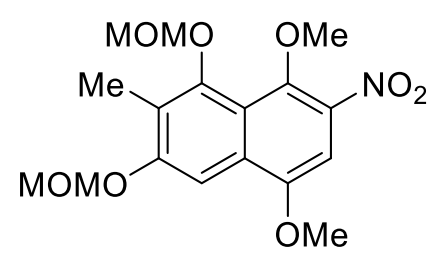

61 (in $\mathrm{CDCl}_{3}, 400 \mathrm{MHz}$ )

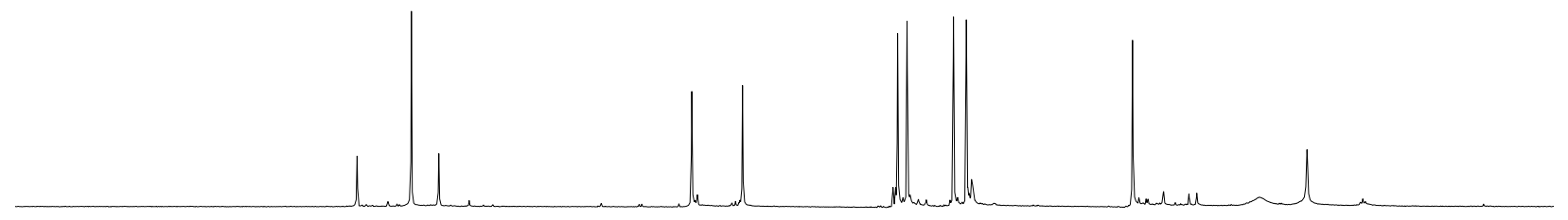

\begin{tabular}{llllllllllllllllllll}
\hline \hline 0 & 9.5 & 9.0 & 8.5 & 8.0 & 7.5 & 7.0 & 6.5 & 6.0 & 5.5 & $\begin{array}{c}5.0 \\
f 1(\mathrm{ppm})\end{array}$ & 4.0 & 3.5 & 3.0 & 2.5 & 2.0 & 1.5 & 1.0 & 0.5 & 0.0
\end{tabular}<smiles>COc1cc2c(OC)cc([N+](=O)[O-])c(OC)c2c(OC)c1OC</smiles>

61 (in $d_{6}$-DMSO, $150 \mathrm{MHz}$ )
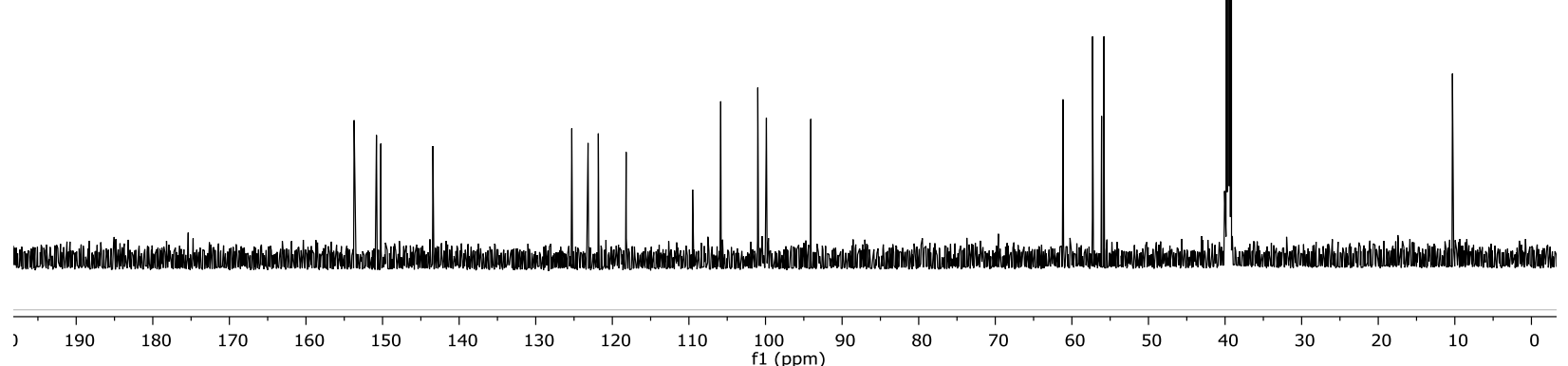


\section{$\mathrm{H}$ and C-NMR ( $\left.\mathrm{CDCl}_{3}\right)$ of compound 63}

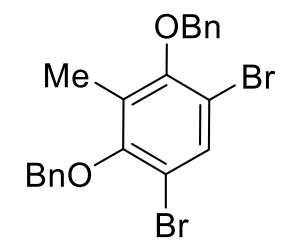

63 (in $\mathrm{CDCl}_{3}, 600 \mathrm{MHz}$ )
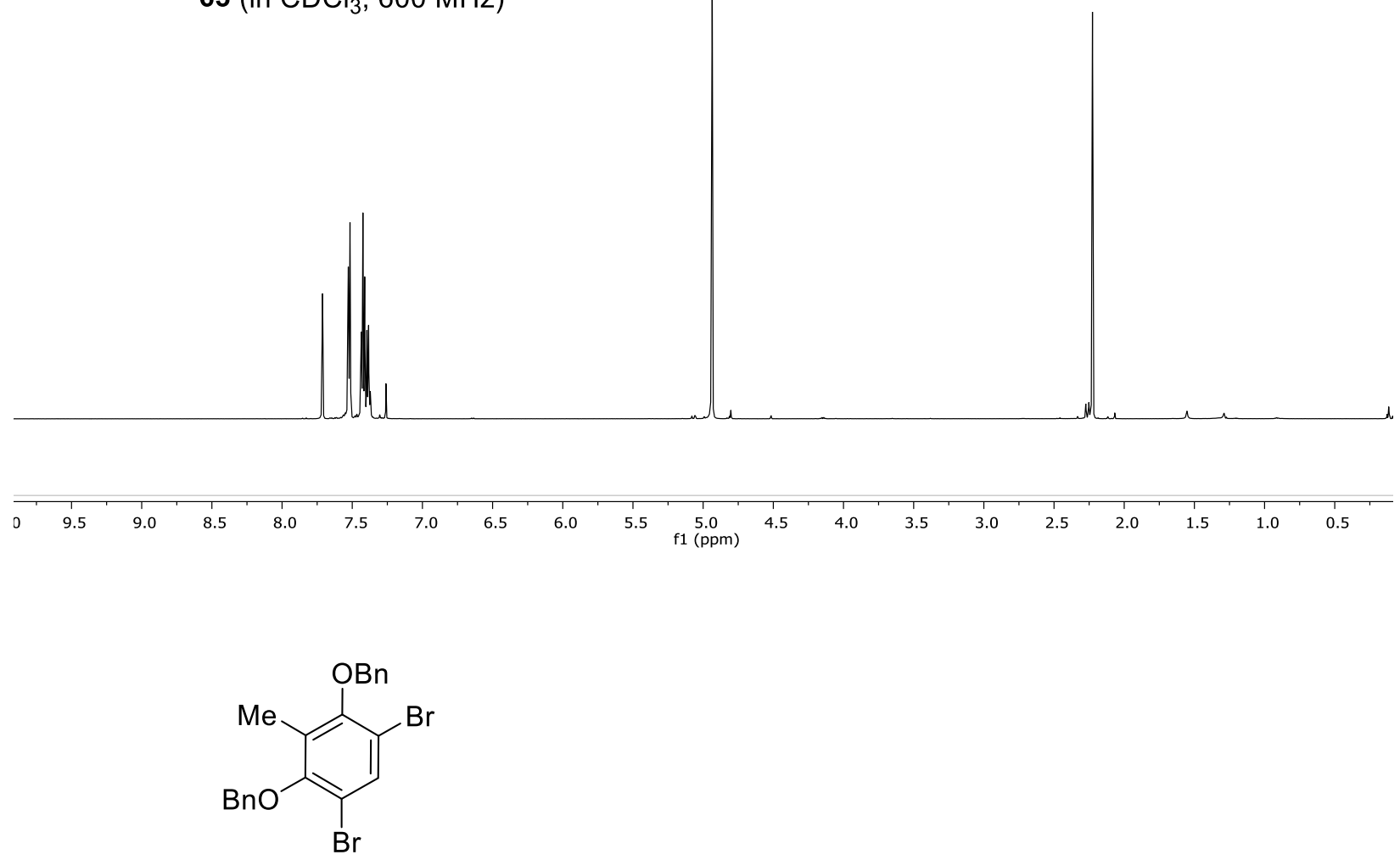

63 (in $\mathrm{CDCl}_{3}, 150 \mathrm{MHz}$ )

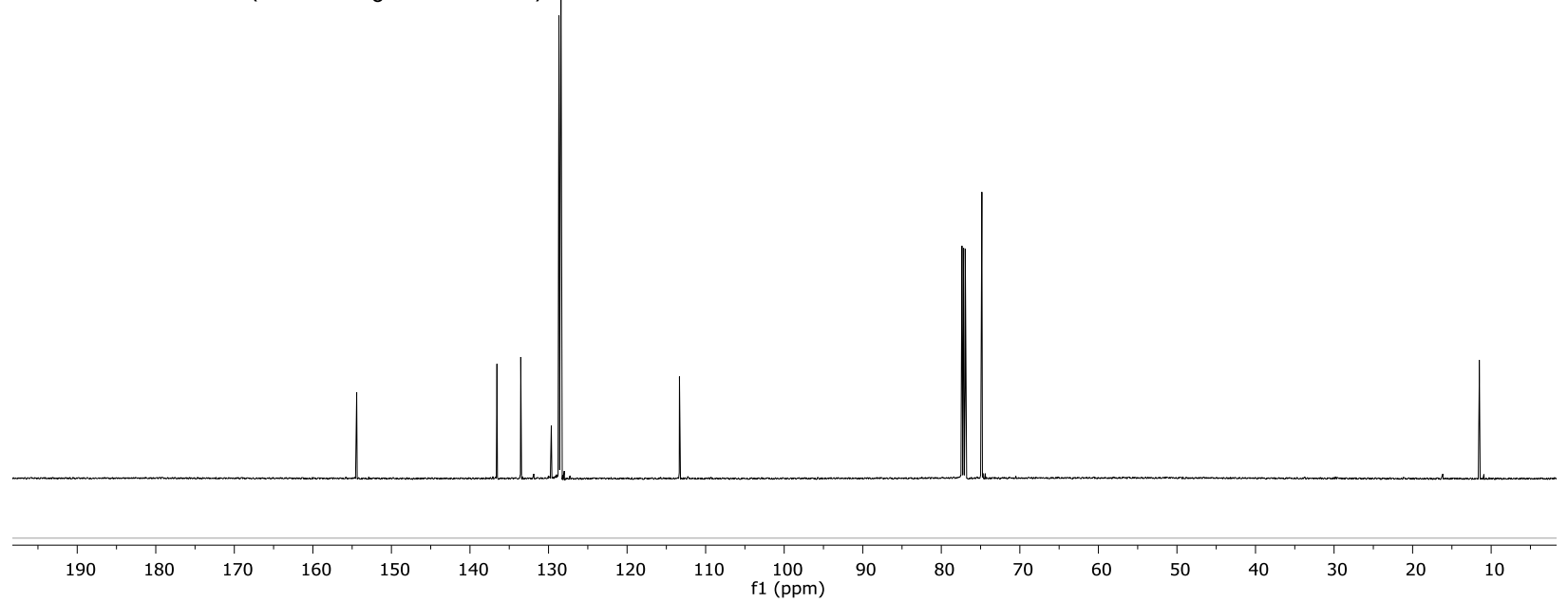




\section{$\mathrm{H}$ and C-NMR ( $\left.\mathrm{CDCl}_{3}\right)$ of compound 64}

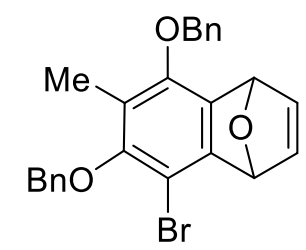

64 (in $\mathrm{CDCl}_{3}, 400 \mathrm{MHz}$ )

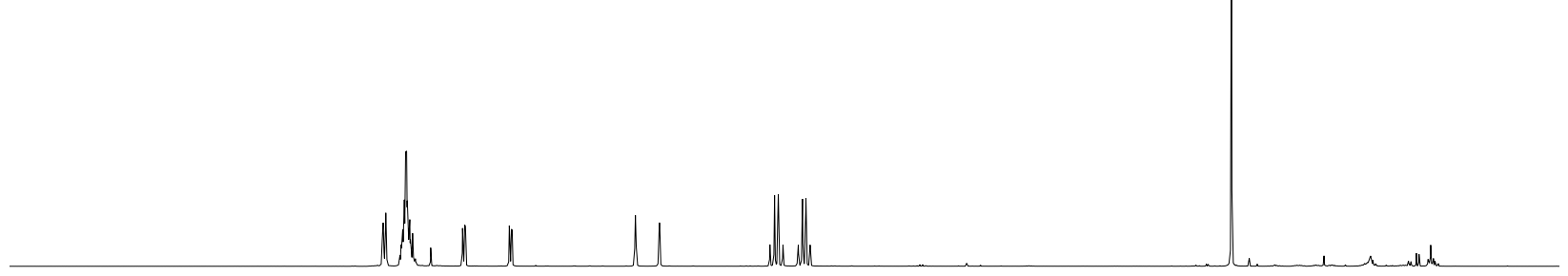

\begin{tabular}{|c|c|c|c|c|c|c|c|c|c|c|c|c|c|c|c|c|c|c|c|}
\hline 0 & 9.5 & 9.0 & 8.5 & 8.0 & 7.5 & 7.0 & 6.5 & 6.0 & 5.5 & $\begin{array}{c}5.0 \\
\text { f1 }(\mathrm{ppm})\end{array}$ & 4.5 & 4.0 & 3.5 & 3.0 & 2.5 & 2.0 & 1.5 & 1.0 & 0.5 \\
\hline
\end{tabular}

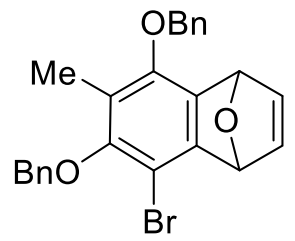

64 (in $\mathrm{CDCl}_{3}, 100 \mathrm{MHz}$ )

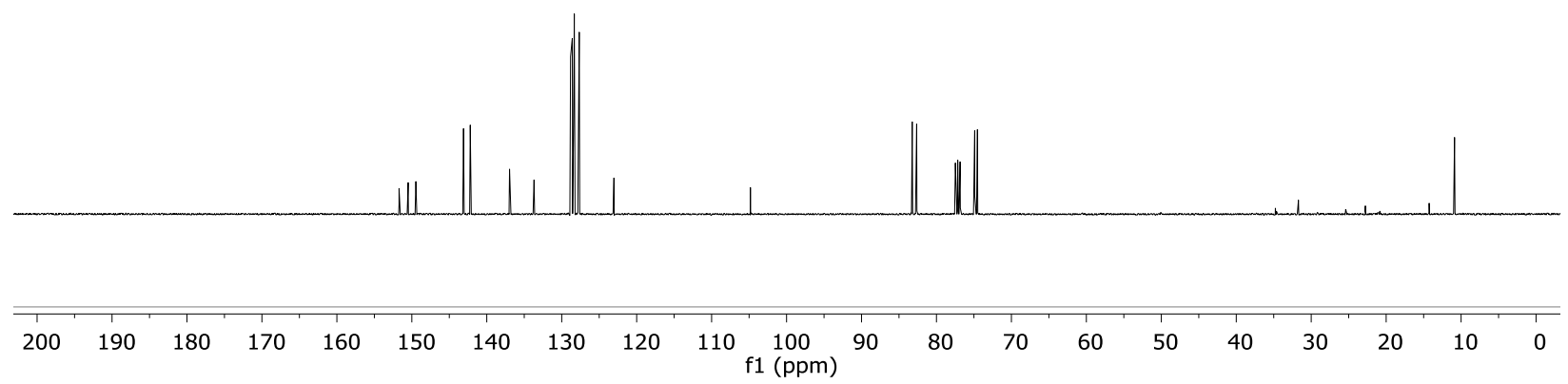




\section{$\mathrm{H}$ and C-NMR $\left(\mathrm{CDCl}_{3}\right)$ of compound $65 \mathrm{a}$}<smiles>Cc1c(OCc2ccccc2)c(Br)c2c(O)cccc2c1OCc1ccccc1</smiles>

65a $\left(\mathrm{CDCl}_{3}, 400 \mathrm{MHz}\right)$

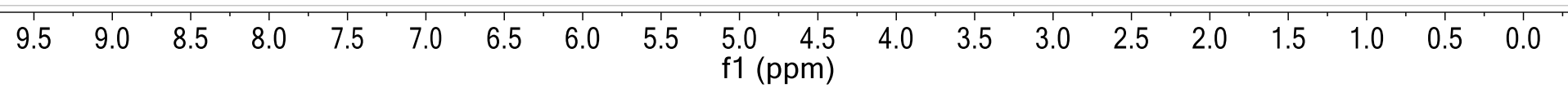<smiles>Cc1c(OCc2ccccc2)c(Br)c2c(O)cccc2c1OCc1ccccc1</smiles>

65a $\left(\mathrm{CDCl}_{3}, 100 \mathrm{MHz}\right)$

$\begin{array}{llllllllllllllllllllllll}220 & 210 & 200 & 190 & 180 & 170 & 160 & 150 & 140 & 130 & 120 & 110 & 100 & 90 & 80 & 70 & 60 & 50 & 40 & 30 & 20 & 10 & 0 & -\end{array}$
$\mathrm{f1}(\mathrm{ppm})$ 


\section{$\mathrm{H}$ and C-NMR ( $\left.\mathrm{CDCl}_{3}\right)$ of compound S9}

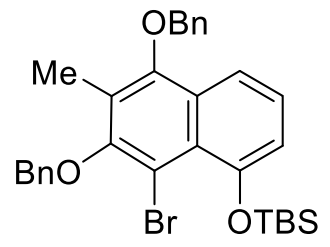

S9 (in $\mathrm{CDCl}_{3}, 600 \mathrm{MHz}$ )
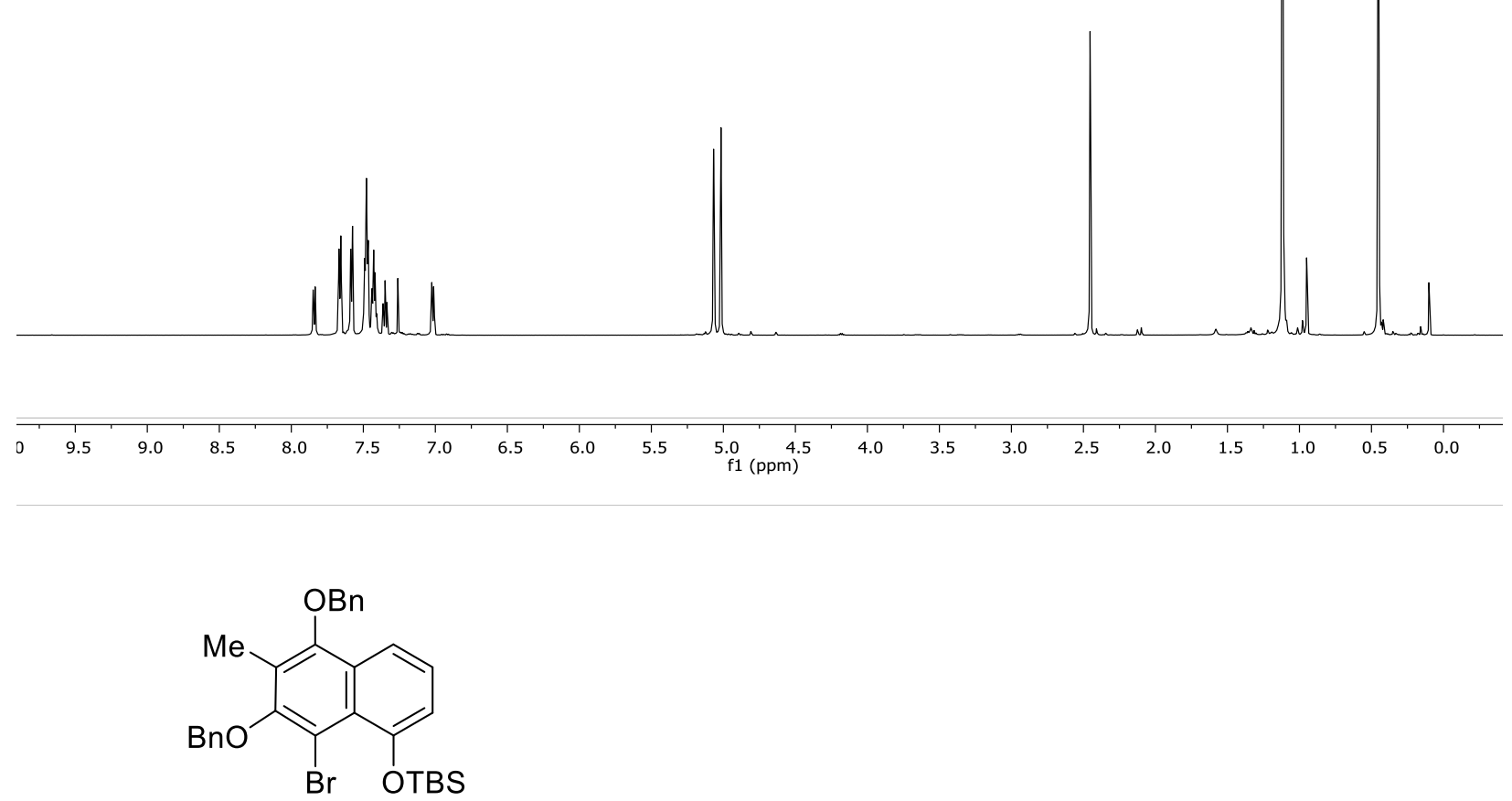

S9 (in $\mathrm{CDCl}_{3}, 150 \mathrm{MHz}$ )

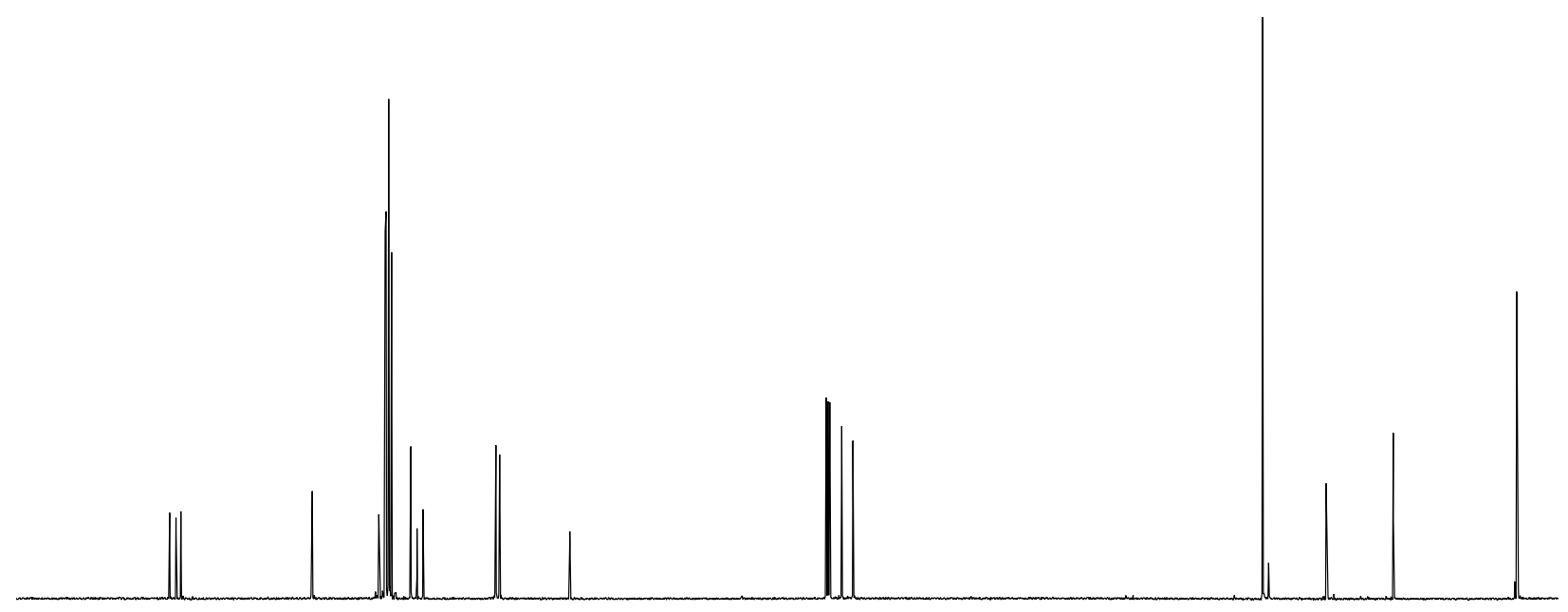

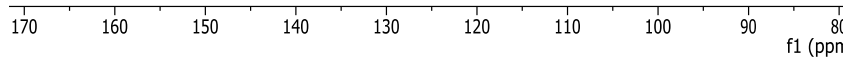




\section{$\mathrm{H}$ and C-NMR $\left(\mathrm{CDCl}_{3}\right)$ of compound $65 \mathrm{~b}$}

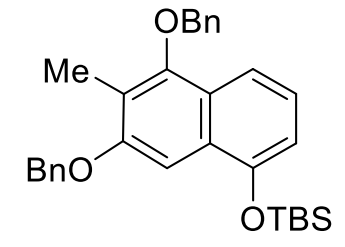

65b (in $\mathrm{CDCl}_{3}, 600 \mathrm{MHz}$ )
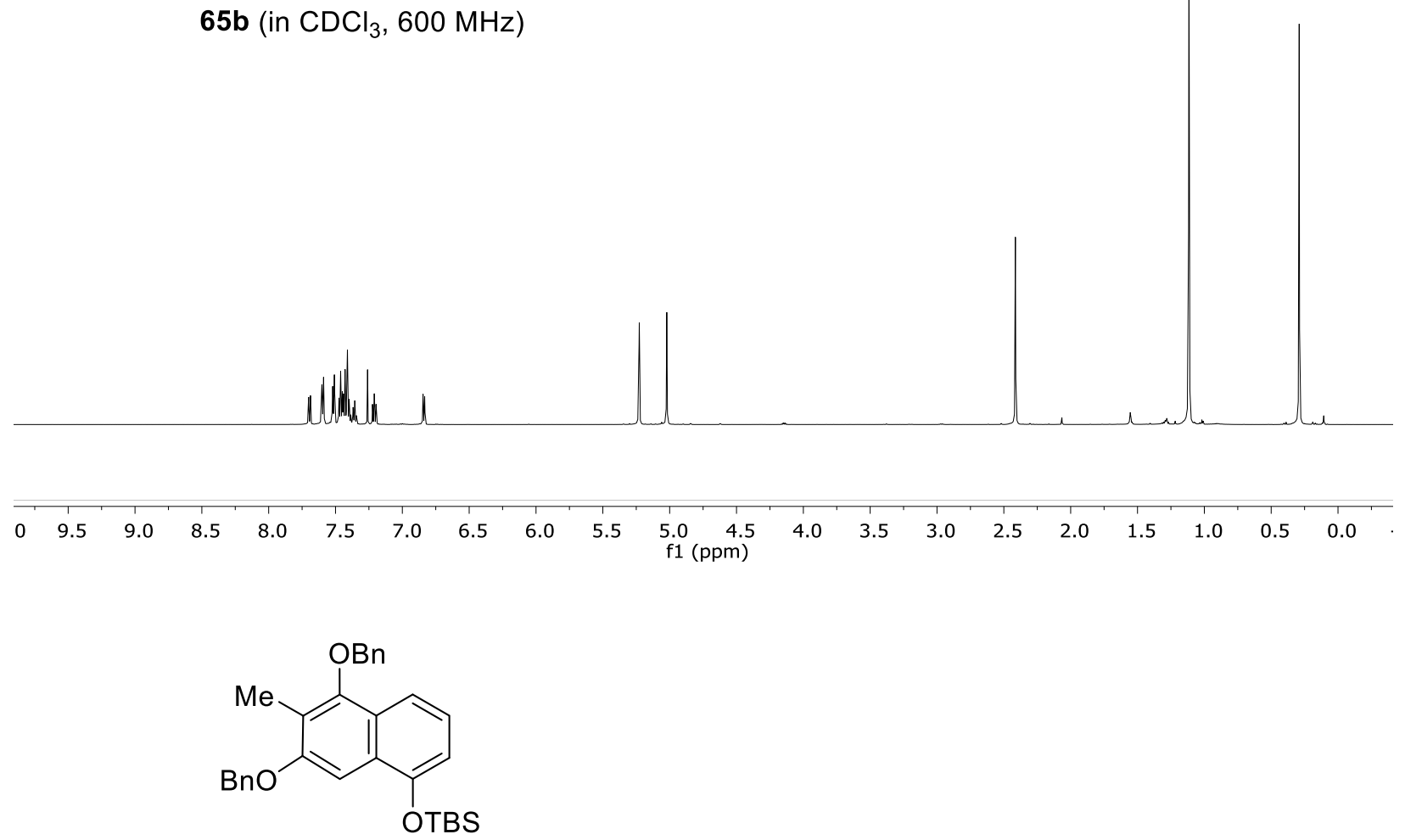

65b (in $\mathrm{CDCl}_{3}, 150 \mathrm{MHz}$ )

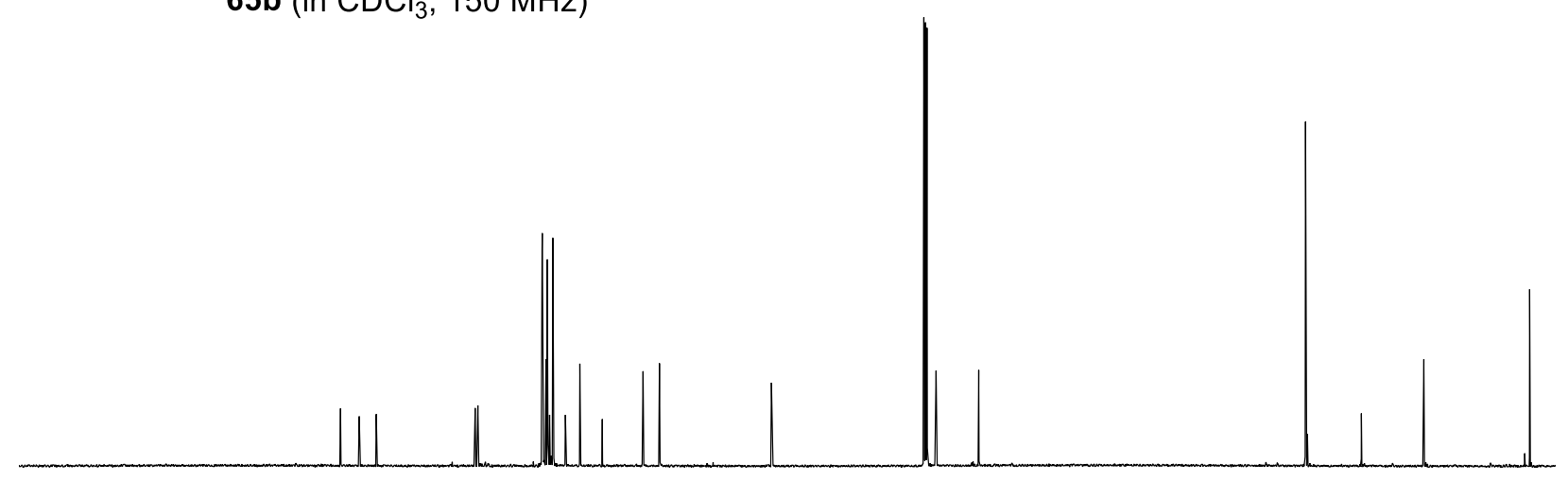


<smiles>Cc1cccc(C(=O)Cc2c(C)c(OCc3ccccc3)c(Br)c3c2C(=O)C=CC3=O)c1</smiles>

\section{$\mathrm{H}$ and C-NMR $\left(\mathrm{CDCl}_{3}\right)$ of compound 66}

$66\left(\mathrm{CDCl}_{3}, 400 \mathrm{MHz}\right)$

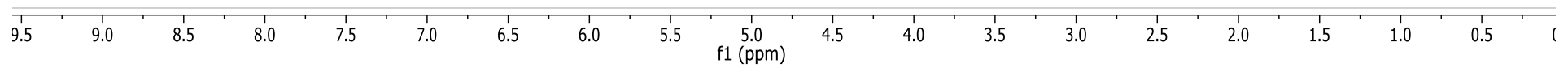<smiles>Cc1c(OCc2ccccc2)c(Br)c2c(c1OCc1ccccc1)C(=O)C=CC2=O</smiles>

$66\left(\mathrm{CDCl}_{3}, 100 \mathrm{MHz}\right)$

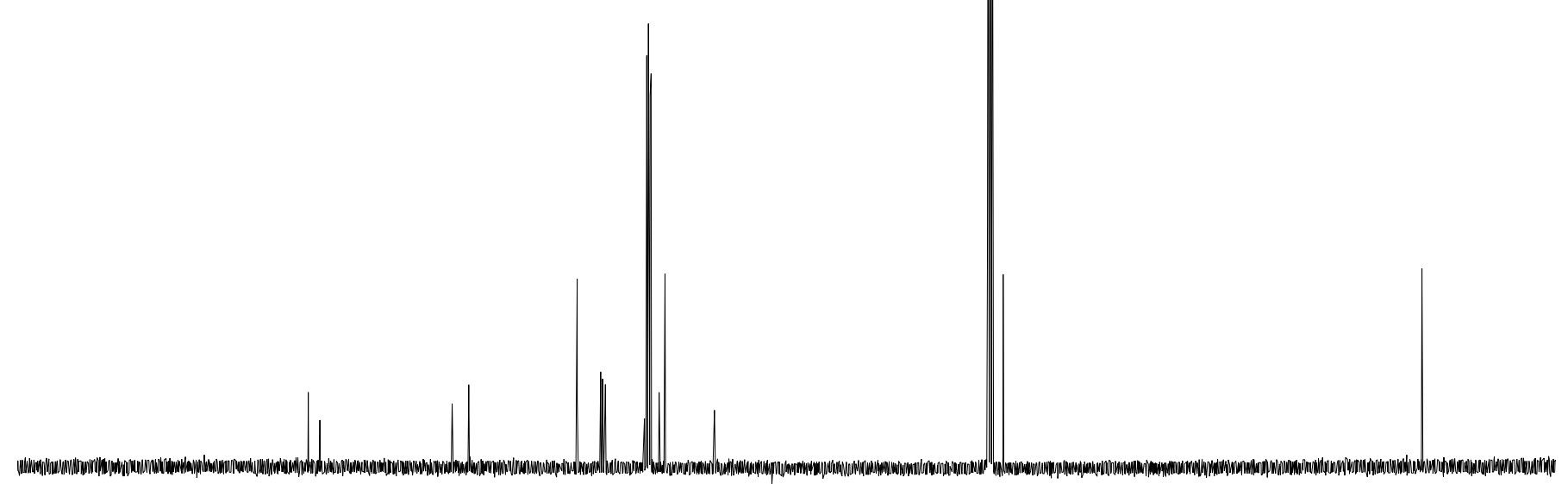

$\begin{array}{lllllllllllllllllllllllll}1 & 1 \\ 220 & 210 & 200 & 190 & 180 & 170 & 160 & 150 & 140 & 130 & 120 & \begin{array}{c}110 \\ \mathrm{f} 1(\mathrm{ppm})\end{array} & 100 & 90 & 80 & 70 & 60 & 50 & 40 & 30 & 20 & 10 & 0\end{array}$




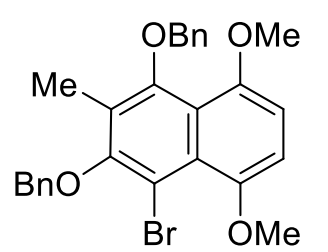

\section{$\mathrm{H}$ and C-NMR $\left(\mathrm{CDCl}_{3}\right)$ of compound 67}

$67\left(\mathrm{CDCl}_{3}, 400 \mathrm{MHz}\right)$

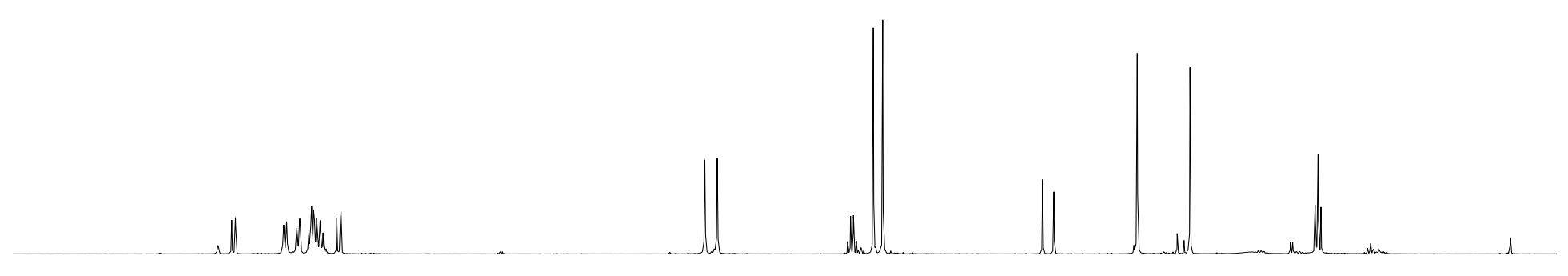

\begin{tabular}{lllllllllllllllllll}
\hline $9^{\prime} .0$ & 8.5 & 8.0 & 7.5 & 7.0 & 6.5 & 6.0 & 5.5 & $\begin{array}{l}5.0 \\
\mathrm{f} 1(\mathrm{ppm})\end{array}$ & 4.5 & 4.0 & 3.5 & 3.0 & 2.5 & 2.0 & 1.5 & 1.0 & 0.5 & 0.0
\end{tabular}<smiles>COc1ccc(OC)c2c(OC)c(C)c(OCc3ccccc3)c(Br)c12</smiles>

$67\left(\mathrm{CDCl}_{3}, 100 \mathrm{MHz}\right)$

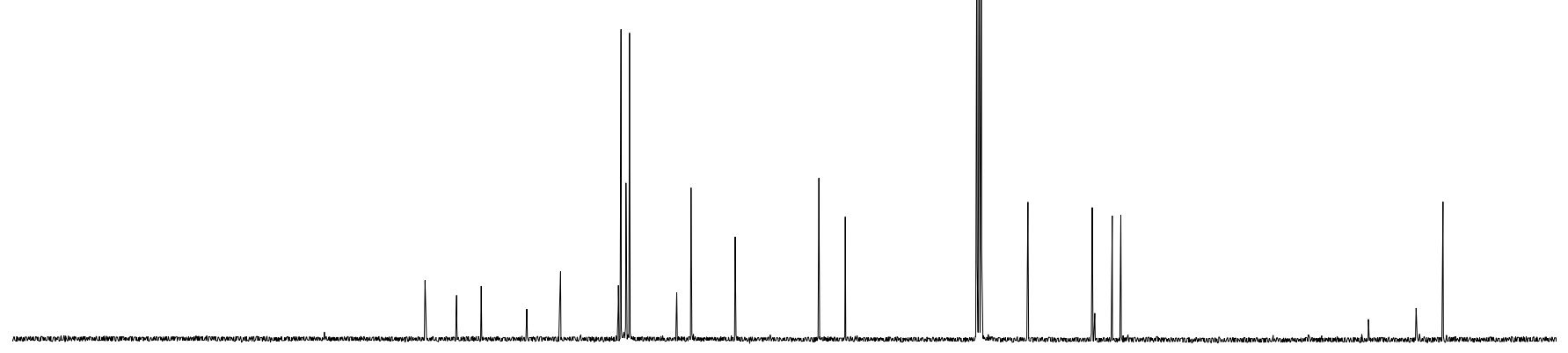

$\begin{array}{lllllllllllllllllllllllll}210 & 200 & 190 & 180 & 170 & 160 & 150 & 140 & 130 & 120 & \begin{array}{l}110 \\ \mathrm{f} 1(\mathrm{ppm})\end{array} & 90 & 80 & 70 & 60 & 50 & 40 & 30 & 20 & 10 & 0\end{array}$




\section{$\mathrm{H}$ and $\mathrm{C}-\mathrm{NMR}\left(\mathrm{CDCl}_{3}\right)$ of compound 68}<smiles>COC(=O)c1c(OC)c(C)c(OCc2ccccc2)c2c(OC)ccc(OC)c12</smiles>

$68\left(\mathrm{CDCl}_{3}, 400 \mathrm{MHz}\right)$

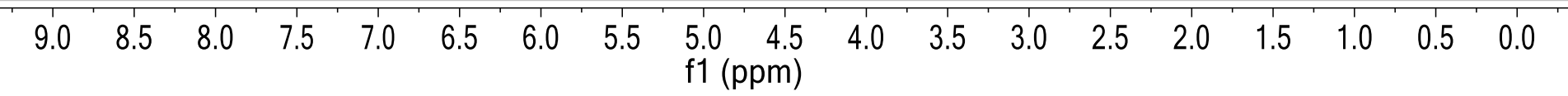<smiles>COC(=O)c1c(OC)c(C)c(OC)c2c(OC)ccc(OC)c12</smiles>

$68\left(\mathrm{CDCl}_{3}, 100 \mathrm{MHz}\right)$

$\begin{array}{lllllllllllllllllllllll}220 & 210 & 200 & 190 & 180 & 170 & 160 & 150 & 140 & 130 & \begin{array}{c}120 \\ \mathrm{f} 1\end{array}(\mathrm{ppm}) & 110 & 100 & 90 & 80 & 70 & 60 & 50 & 40 & 30 & 20 & 10 & 0\end{array}$ 


\section{$\mathrm{H}$ and C-NMR $\left(\mathrm{CDCl}_{3}\right)$ of compound 69}<smiles>COC(=O)c1c(O)c(C)c(O)c2c(OC)ccc(OC)c12</smiles>

$69\left(600 \mathrm{MHz}\right.$, in $\left.\mathrm{CDCl}_{3}\right)$
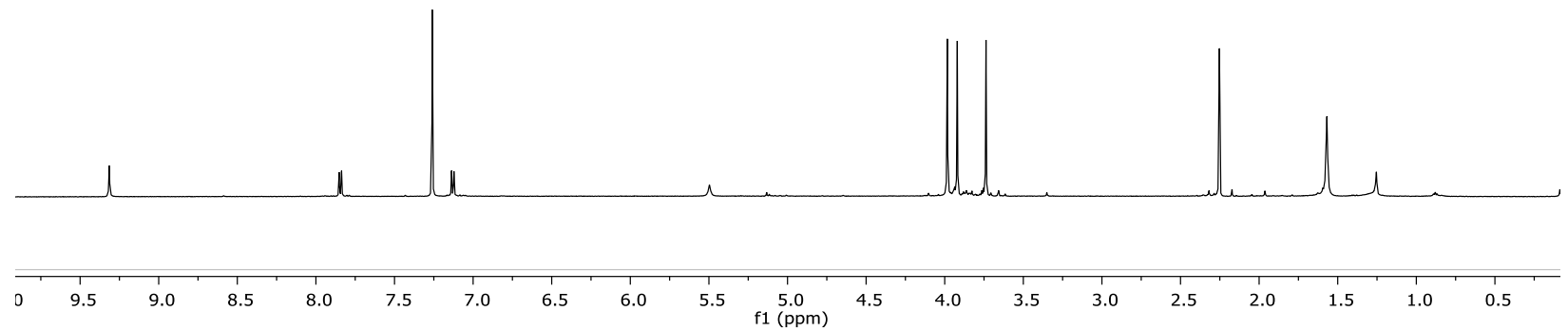<smiles>COC(=O)c1c(O)c(C)c(O)c2c(OC)ccc(OC)c12</smiles>

$69\left(150 \mathrm{MHz}\right.$, in $\left.\mathrm{CDCl}_{3}\right)$

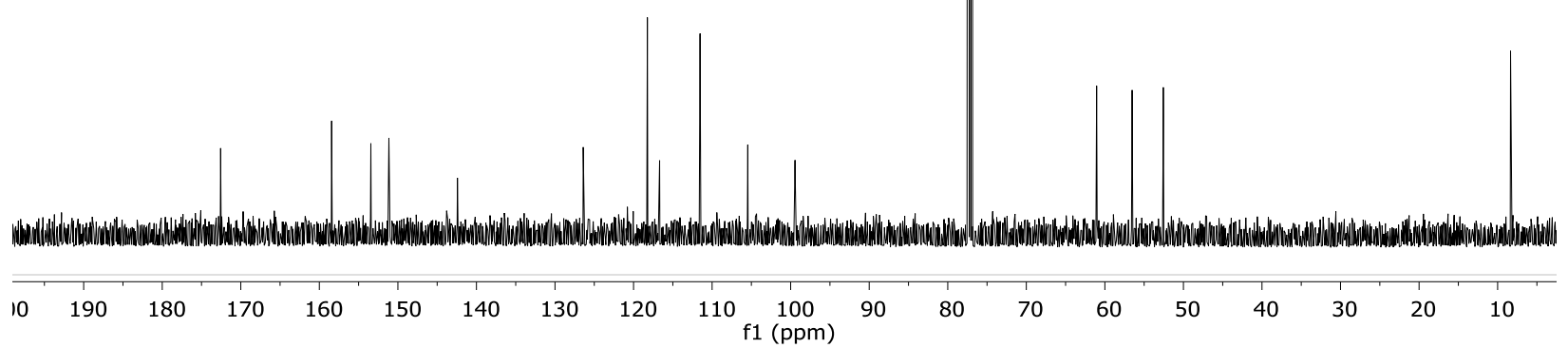




\section{$\mathrm{H}$ and C-NMR $\left(\mathrm{CDCl}_{3}\right)$ of compound 72}

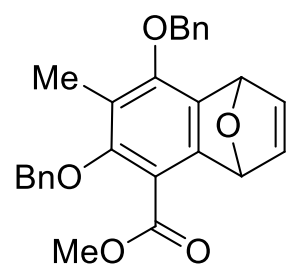

$72\left(\mathrm{CDCl}_{3}, 400 \mathrm{MHz}\right)$

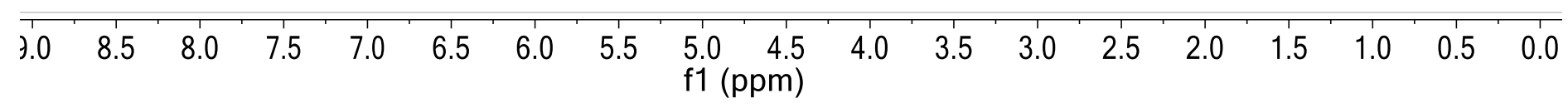<smiles>COC(=O)c1c(OC)c(OC)c(Br)c2c1C1CCC2O1</smiles>

$72\left(\mathrm{CDCl}_{3}, 100 \mathrm{MHz}\right)$

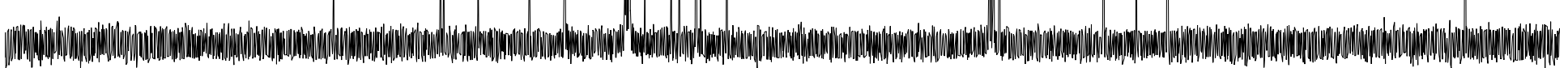

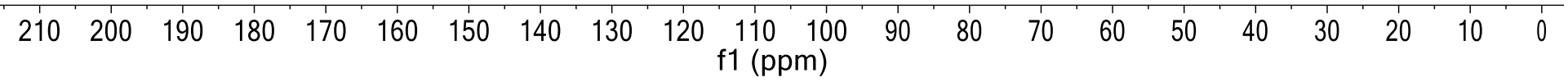




\section{$\mathrm{H}$ and C-NMR ( $\left.\mathrm{CDCl}_{3}\right)$ of compound 75}

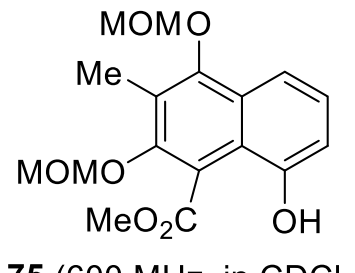

$75\left(600 \mathrm{MHz}\right.$, in $\left.\mathrm{CDCl}_{3}\right)$
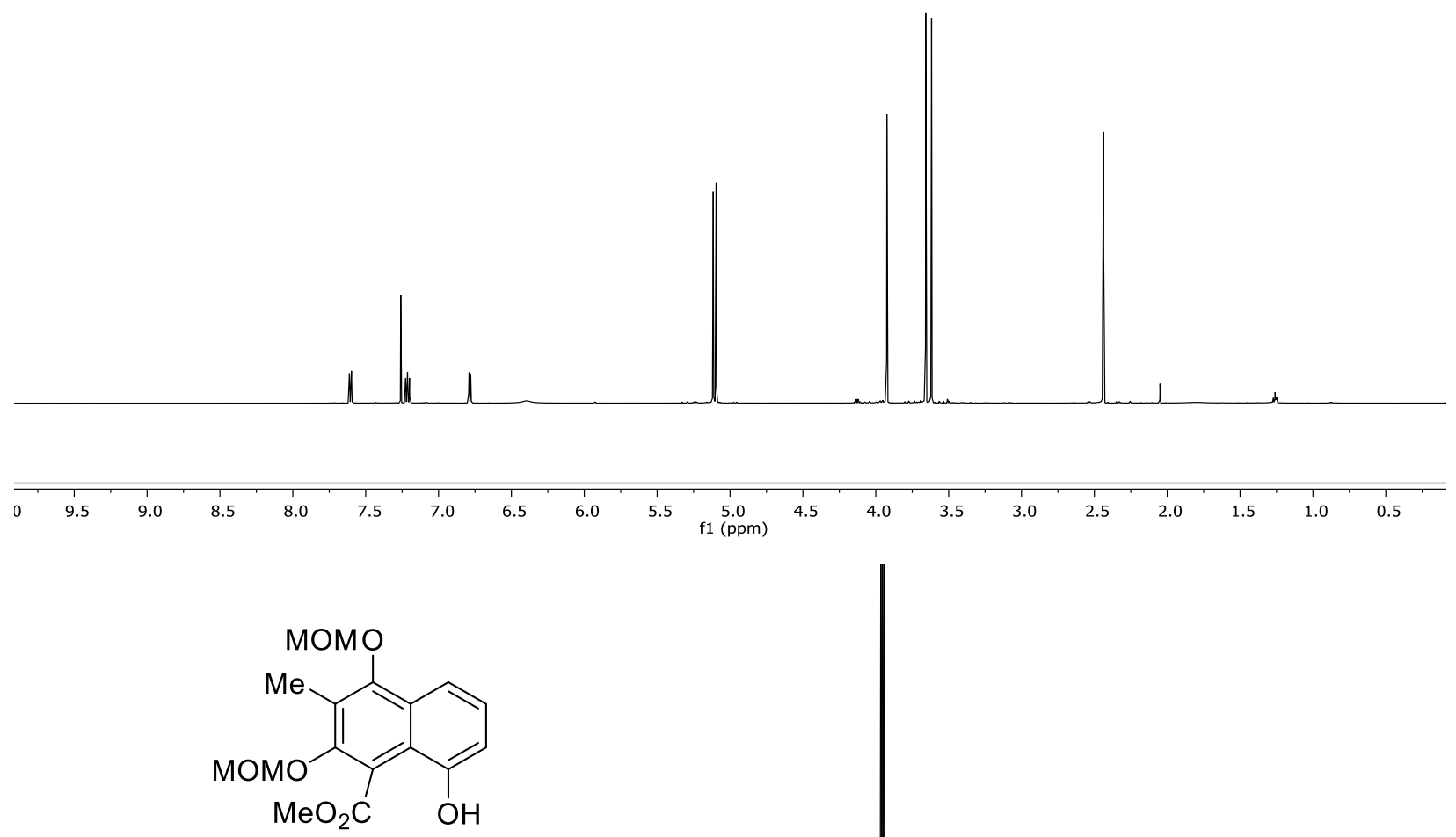

$75\left(150 \mathrm{MHz}\right.$, in $\left.\mathrm{CDCl}_{3}\right)$
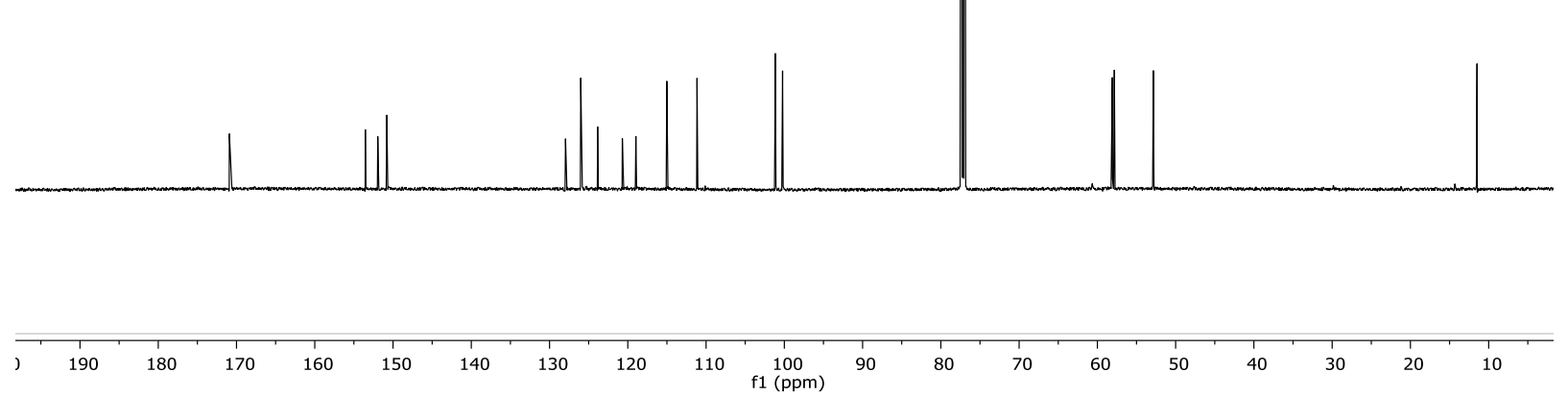


\section{$\mathrm{H}$ and C-NMR ( $\left.\mathrm{CDCl}_{3}\right)$ of compound 74}

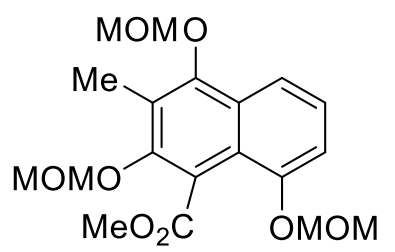

$74\left(600 \mathrm{MHz}\right.$, in $\left.\mathrm{CDCl}_{3}\right)$
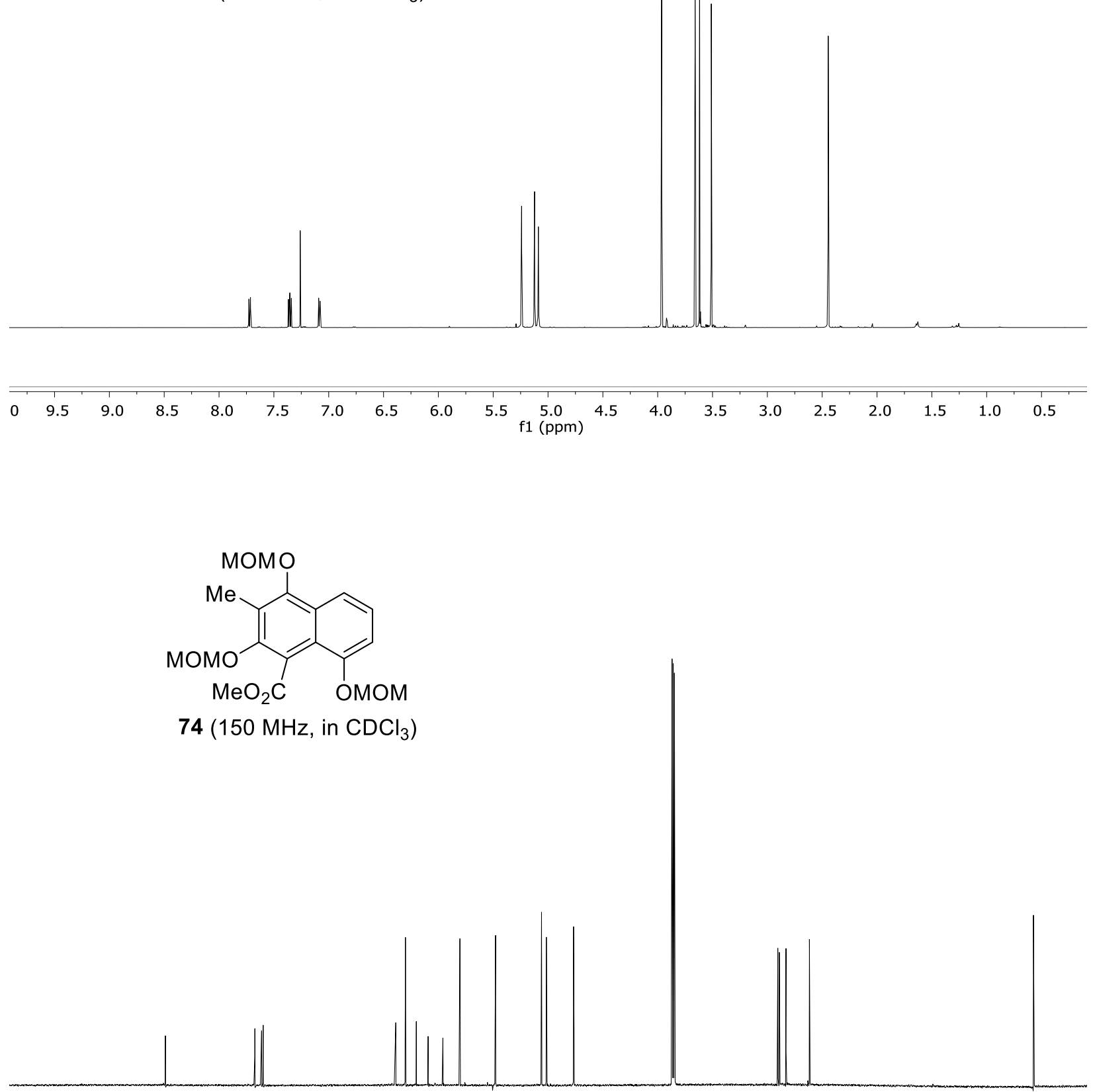

$\begin{array}{llll}190 & 180 & 170 & 160\end{array}$

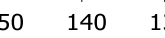

$130 \quad 12$

$\begin{array}{llllllllll}100 & 100 & 80 & 70 & 60 & 50 & 40 & 30 & 20 & 10 \\ \mathrm{f} 1(\mathrm{ppm}) & 90 & & & & \end{array}$




\section{$\mathrm{H}$ and C-NMR ( $\left.\mathrm{CDCl}_{3}\right)$ of compound 76}

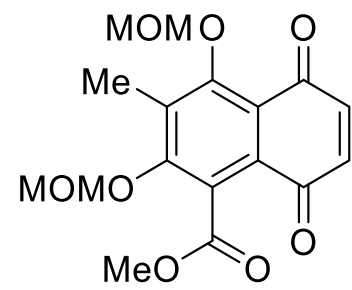

76 (in $\mathrm{CDCl}_{3}, 600 \mathrm{MHz}$ )

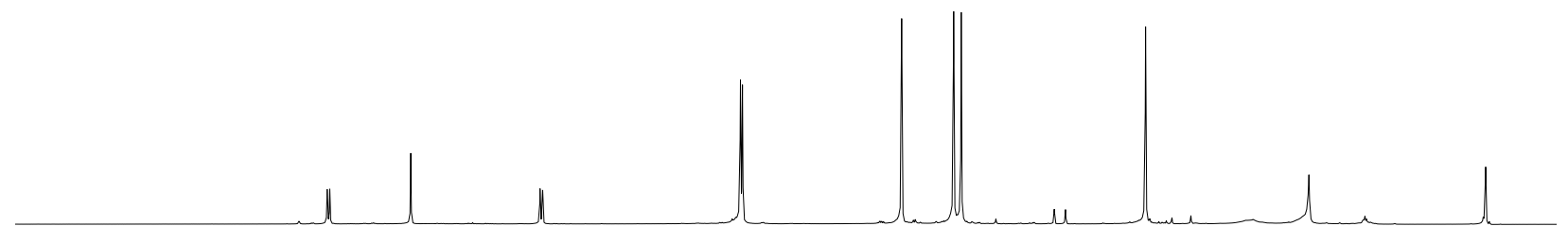

\begin{tabular}{llllllllllllllllllll}
\hline 0 & 9.5 & 9.0 & 8.5 & 8.0 & 7.5 & 7.0 & 6.5 & 6.0 & 5.5 & $\begin{array}{c}5.0 \\
f 1(\mathrm{ppm})\end{array}$ & 4.5 & 3.5 & 3.0 & 2.5 & 2.0 & 1.5 & 1.0 & 0.5 & 0.0
\end{tabular}.

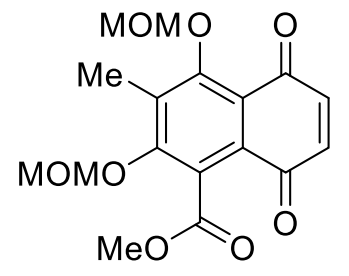

76 (in $\mathrm{CDCl}_{3}, 150 \mathrm{MHz}$ )

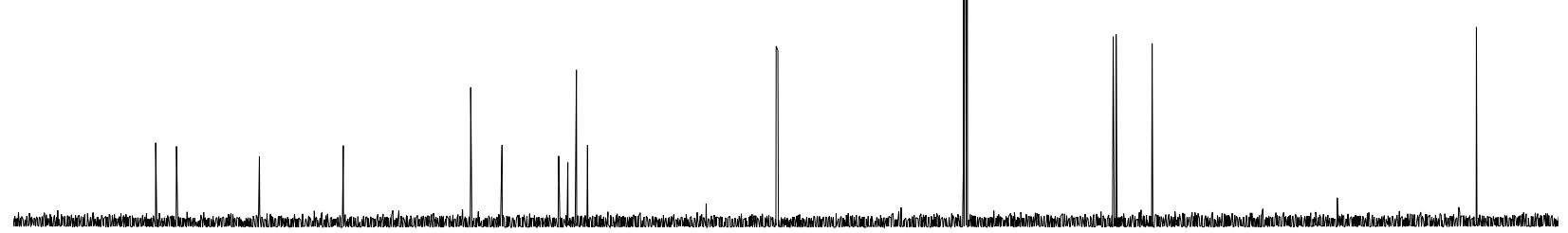

$\begin{array}{llllllllllllllllllll}0 & 190 & 180 & 170 & 160 & 150 & 140 & 130 & 120 & 110 & \begin{array}{c}100 \\ \mathrm{f} 1(\mathrm{ppm})\end{array} & 90 & 80 & 70 & 60 & 50 & 40 & 30 & 20 & 10\end{array}$ 


\section{$\mathrm{H}$ and C-NMR ( $\left.\mathrm{CDCl}_{3}\right)$ of compound 70}

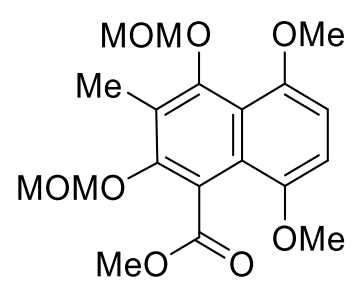

70 (in $\mathrm{CDCl}_{3}, 400 \mathrm{MHz}$ )
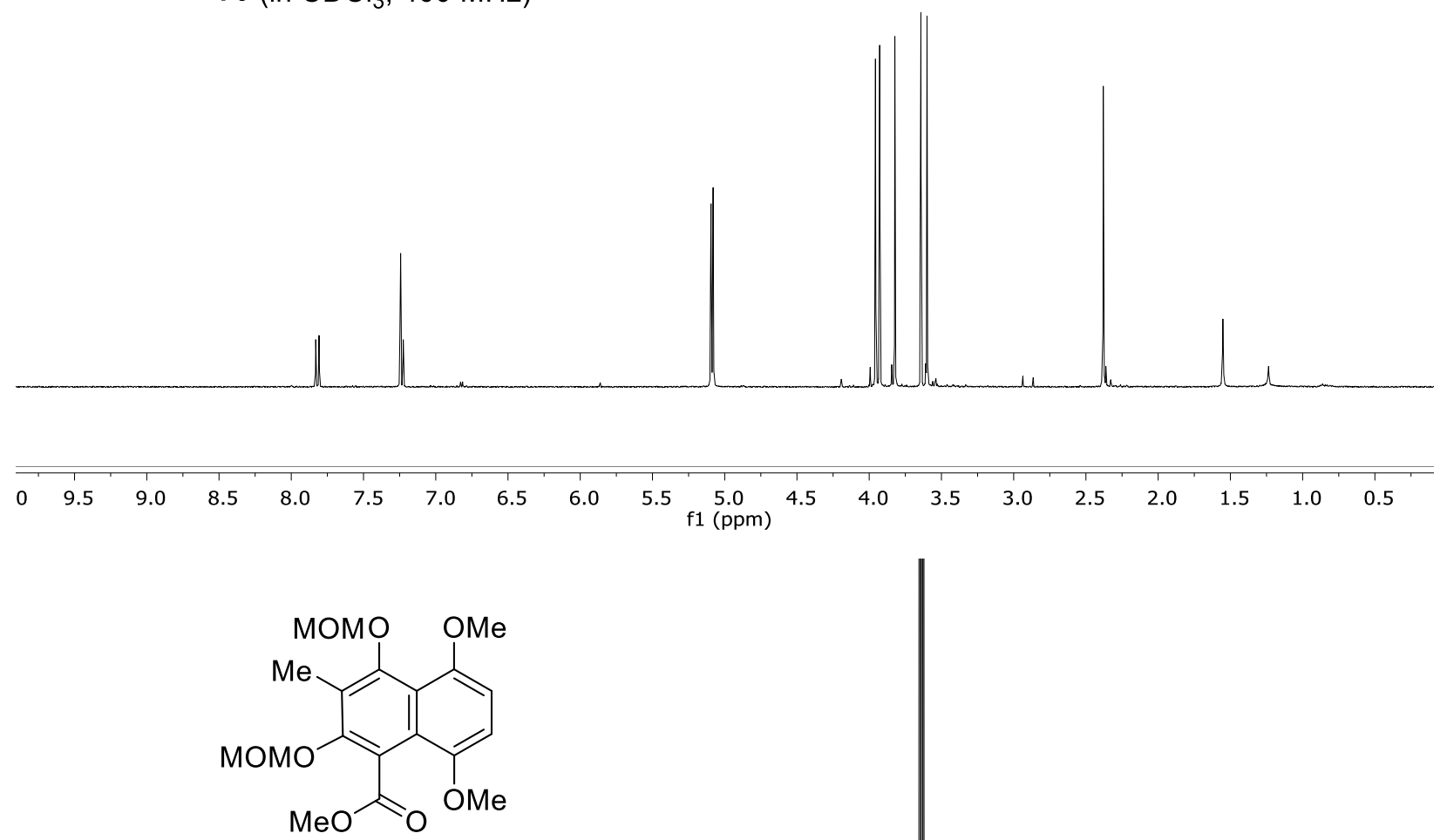

70 (in $\mathrm{CDCl}_{3}, 100 \mathrm{MHz}$ )

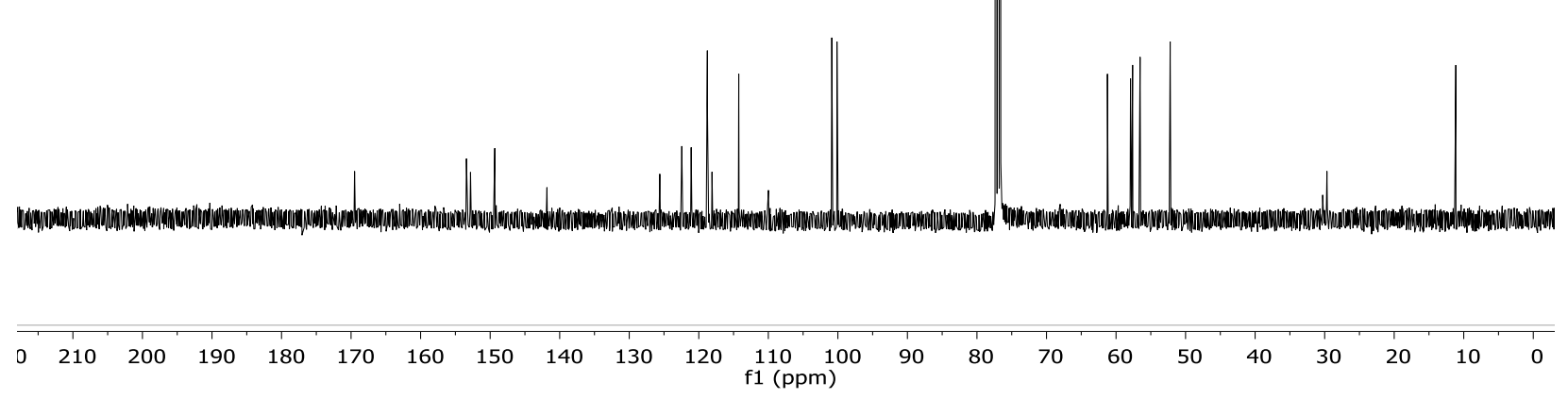




\section{$\mathrm{H}$ and C-NMR $\left(\mathrm{CD}_{3} \mathrm{COCD}_{3}\right)$ of compound 77}<smiles>COC(=O)c1c(OC)c(OC)c(OC)c2c(OC)c([N+](=O)[O-])cc(OC)c12</smiles>

77 (in $\mathrm{CD}_{3} \mathrm{COCD}_{3}, 125 \mathrm{MHz}$ )
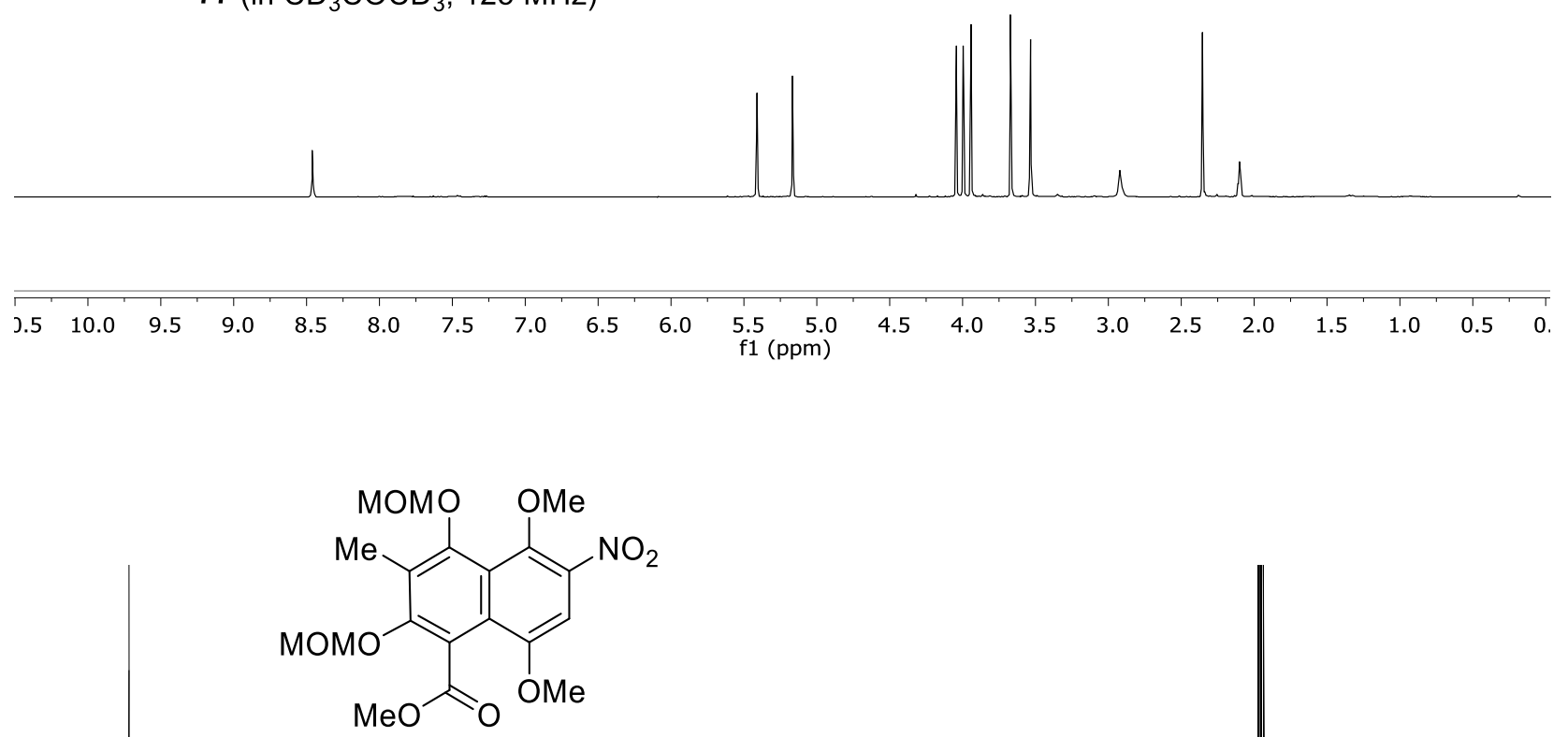

77 (in $\mathrm{CD}_{3} \mathrm{COCD}_{3}, 500 \mathrm{MHz}$ )

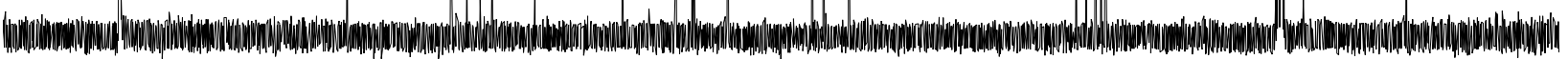

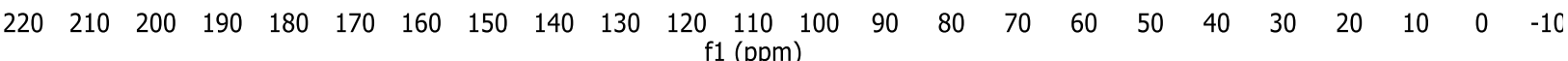




\section{H-NMR $\left(\mathrm{CDCl}_{3}\right)$ of compound 81 (crude)}

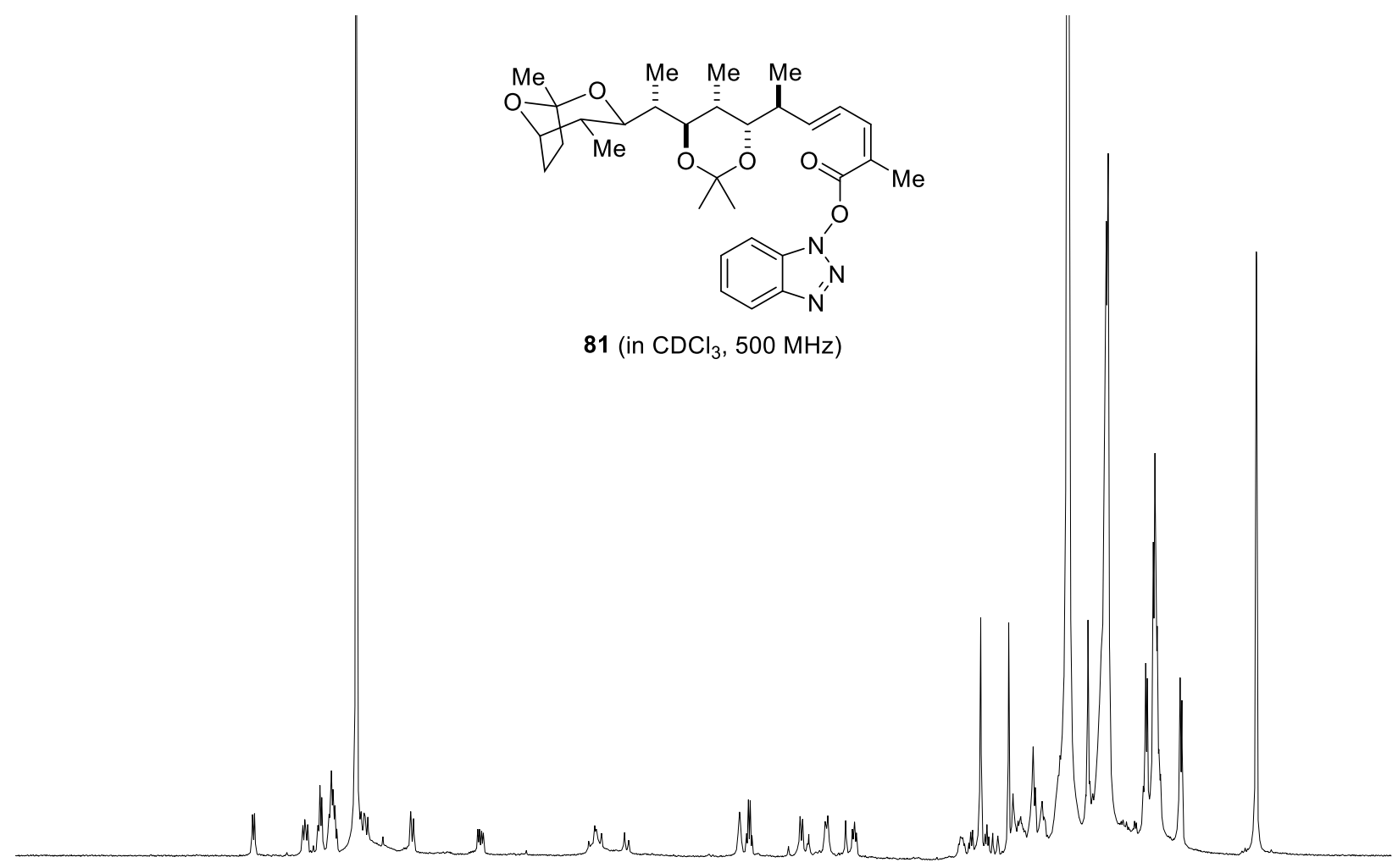

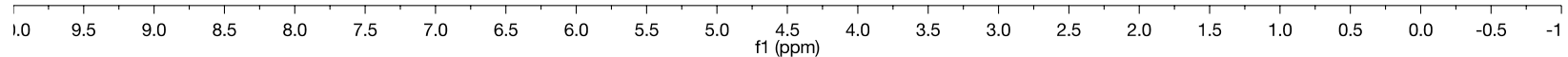




\section{$\mathrm{H}$ and C-NMR $\left(\mathrm{CD}_{3} \mathrm{OD}\right)$ of compound 84}
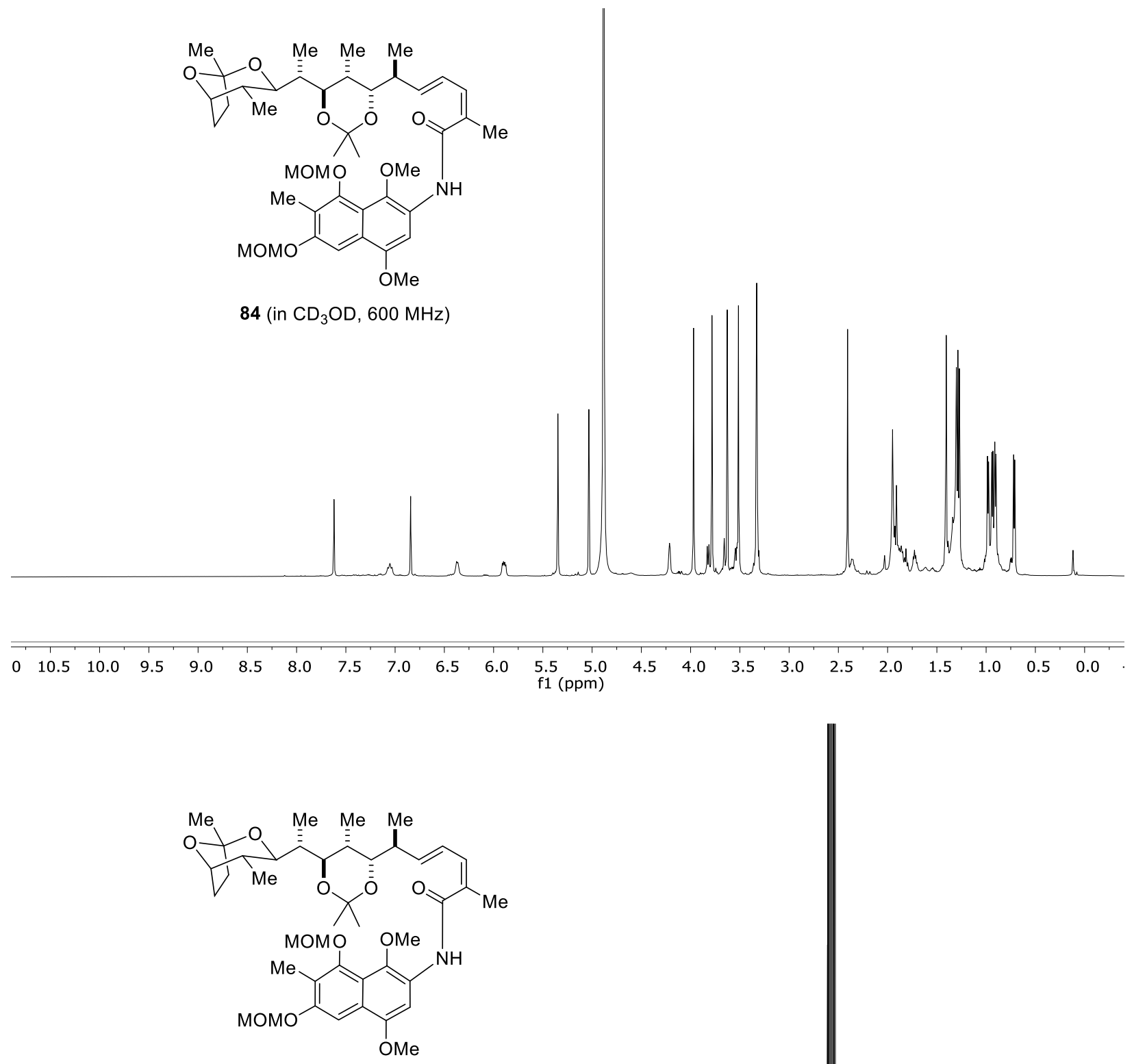

84 (in $\mathrm{CD}_{3} \mathrm{OD}, 150 \mathrm{MHz}$ )
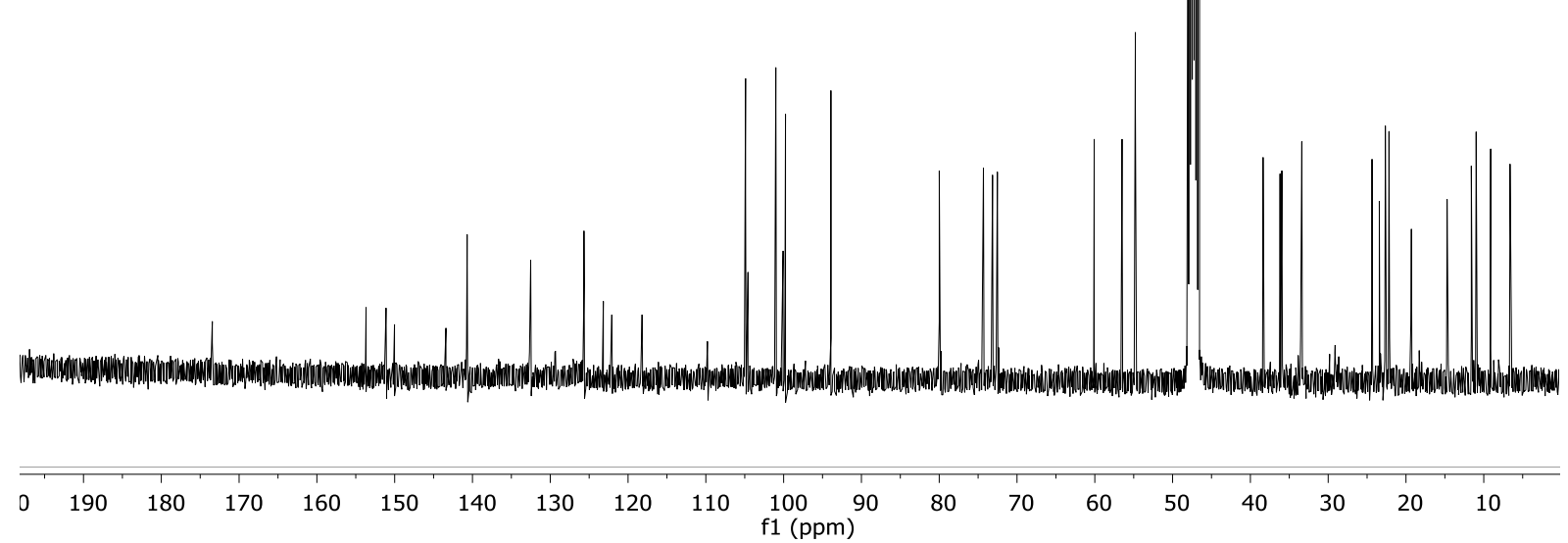
H-NMR ( $\left.\mathrm{CD}_{3} \mathrm{OD}\right)$ of Salinisporamycin (2)

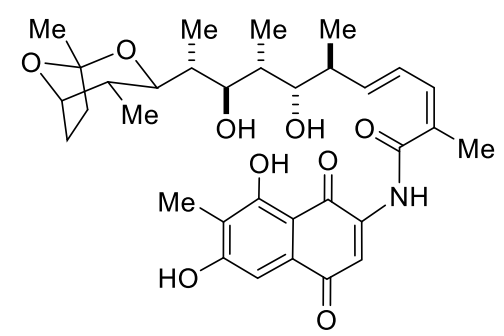

Salinisporamycin (synthetic 2, in $\mathrm{CD}_{3} \mathrm{OD}, 600 \mathrm{MHz}$ )

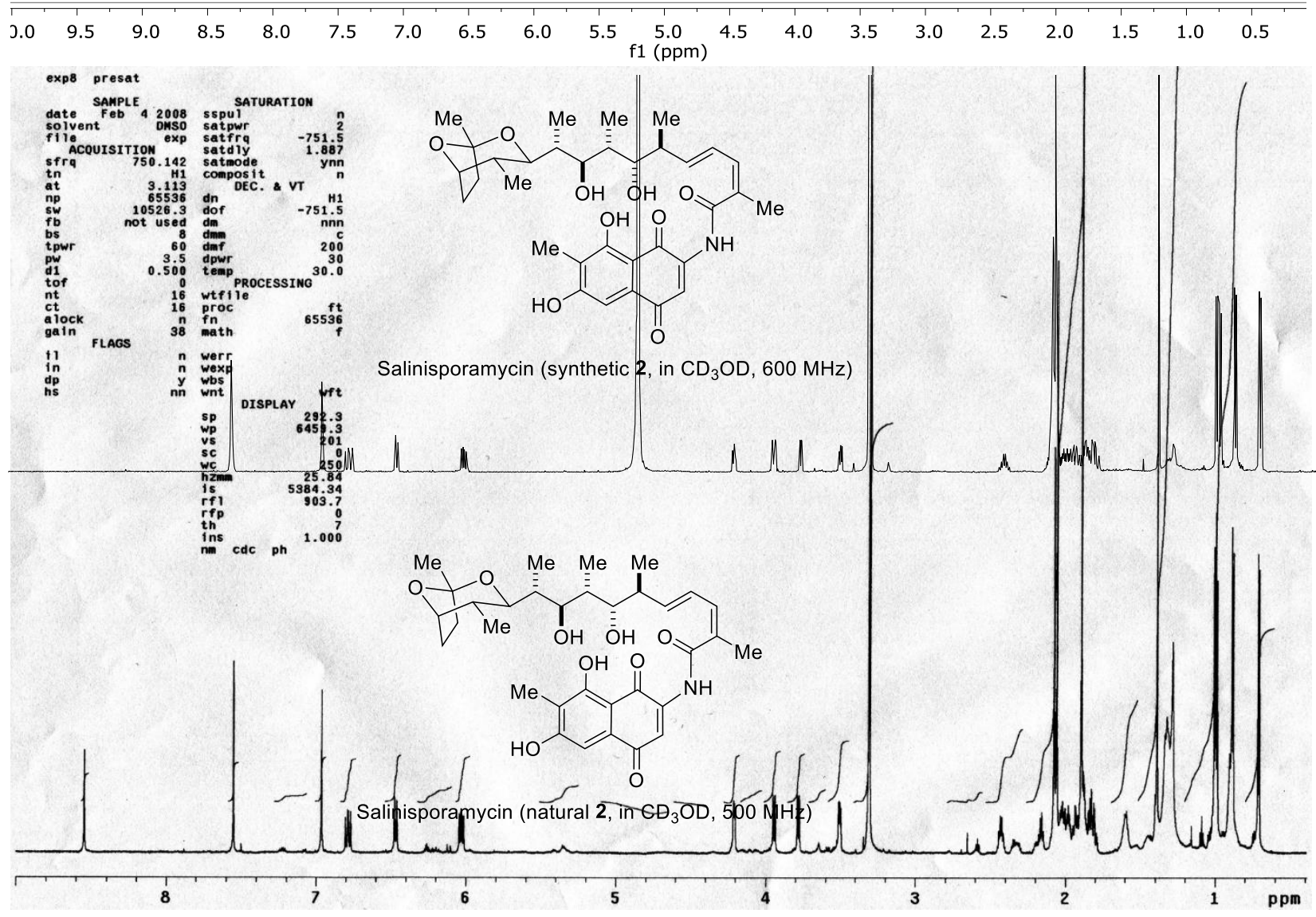




\section{C-NMR $\left(\mathrm{CD}_{3} \mathrm{OD}\right)$ of Salinisporamycin (2)}

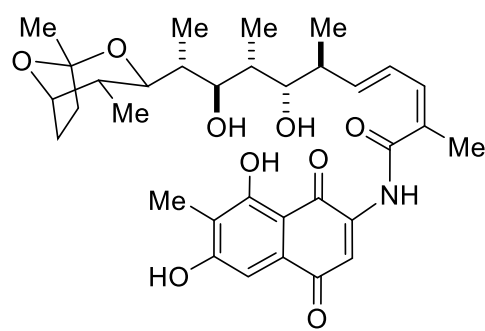

Salinisporamycin (2, synthetic,

in $\mathrm{CD}_{3} \mathrm{OD}, 125 \mathrm{MHz}$ )
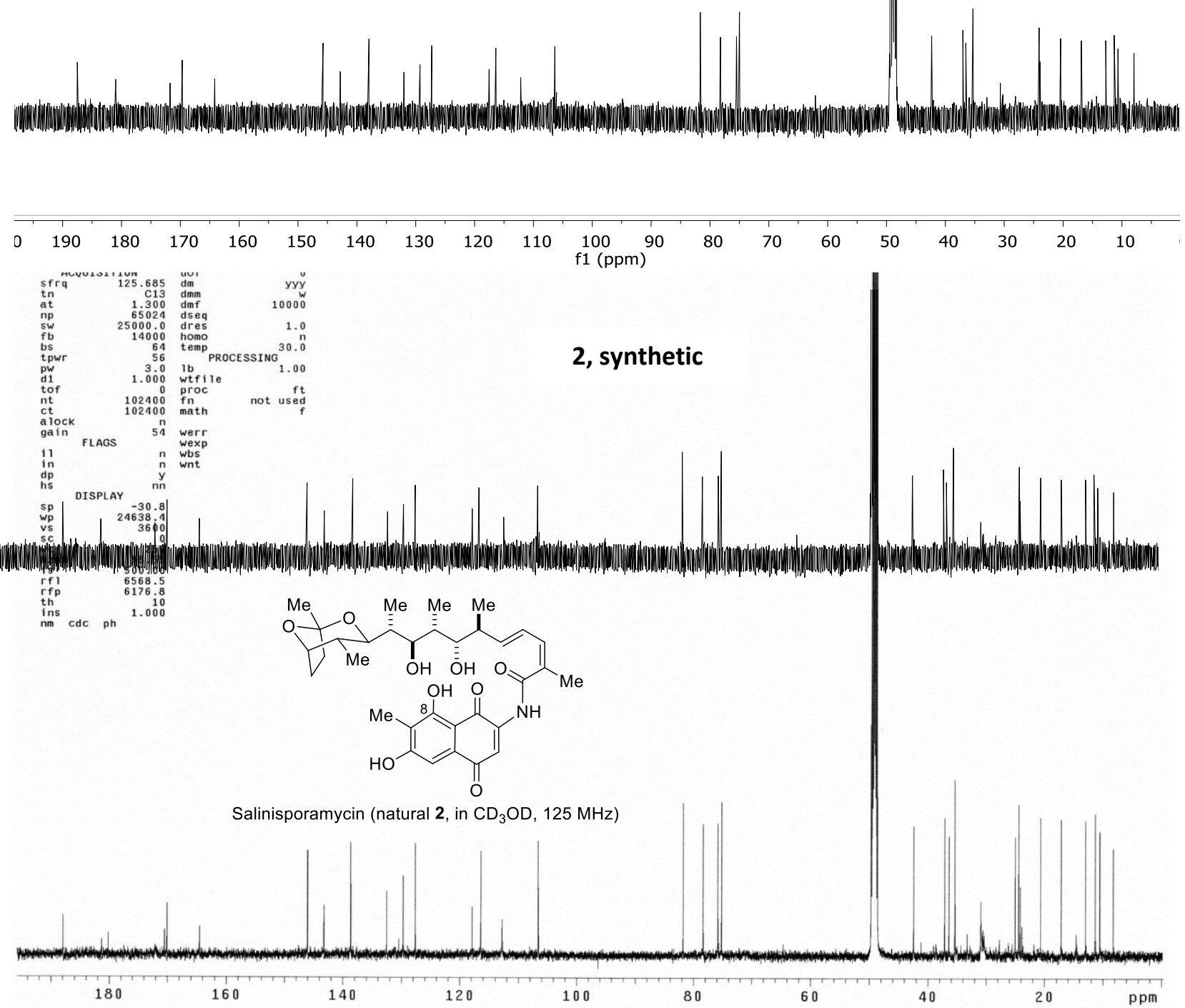


\section{$\mathrm{H}$ and C-NMR $\left(\mathrm{CD}_{3} \mathrm{OD}\right)$ of compound 85}
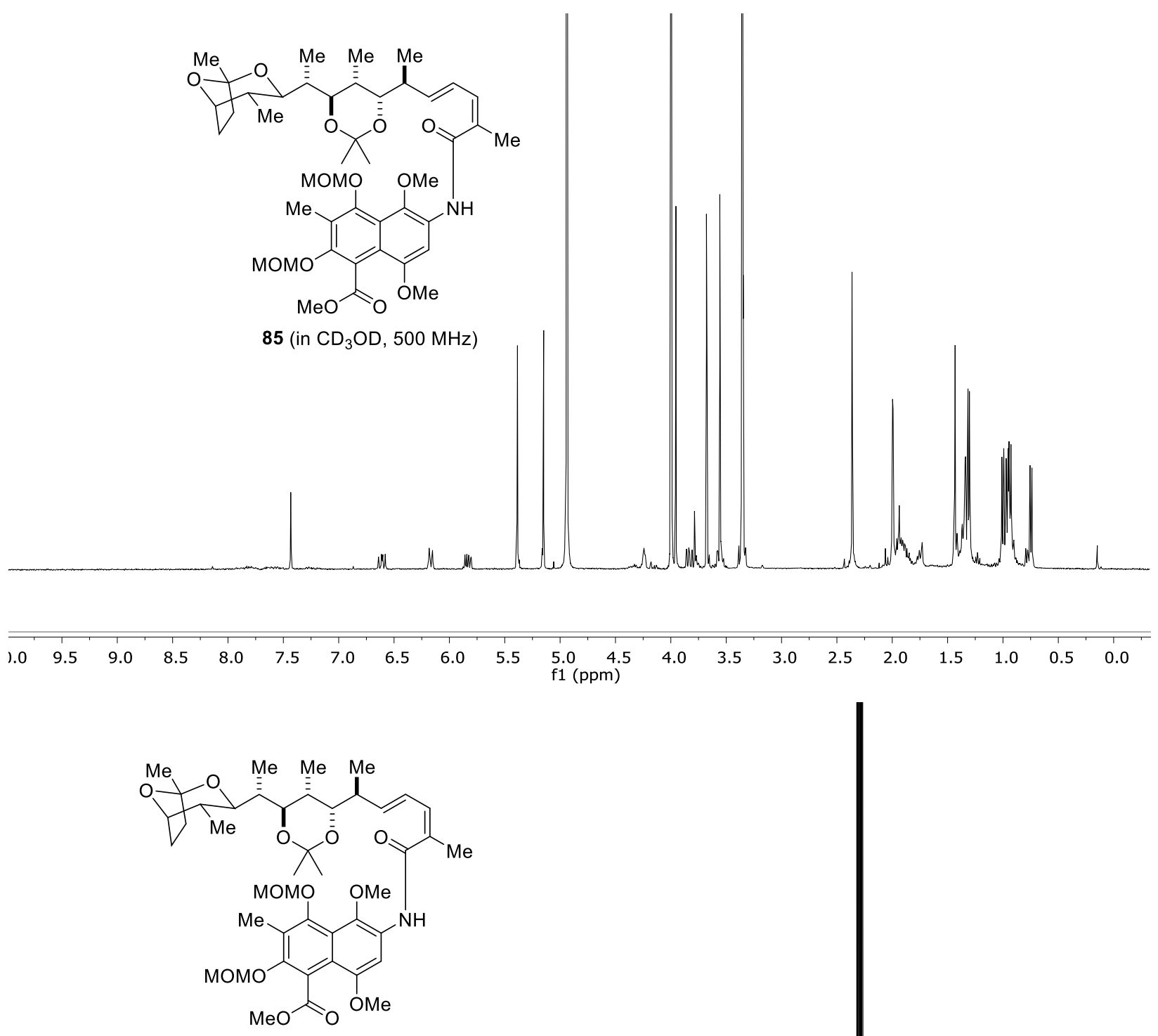

85 (in $\mathrm{CD}_{3} \mathrm{OD}, 125 \mathrm{MHz}$ )

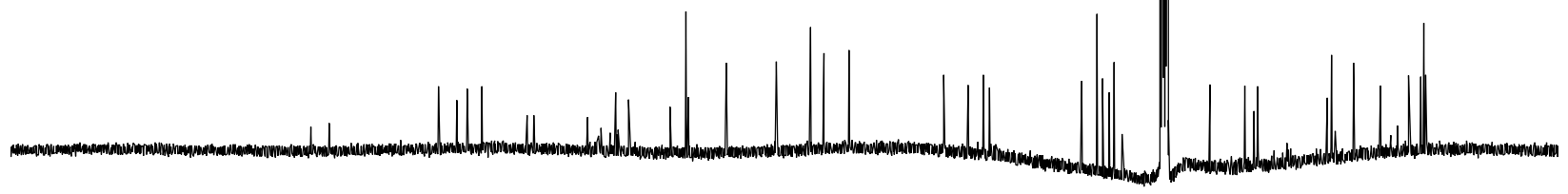

$\begin{array}{lllllllllllll}20 & 210 & 200 & 190 & 180 & 170 & 160 & 150 & 140 & 130 & 120 & 110 & 100\end{array}$

$\begin{array}{lllllll}60 & 50 & 40 & 30 & 20 & 10 & 0\end{array}$ 


\section{$\mathrm{H}$ and -NMR ( $\left.\mathrm{CD}_{3} \mathrm{OD}\right)$ of Rifsaliniketal (9)}

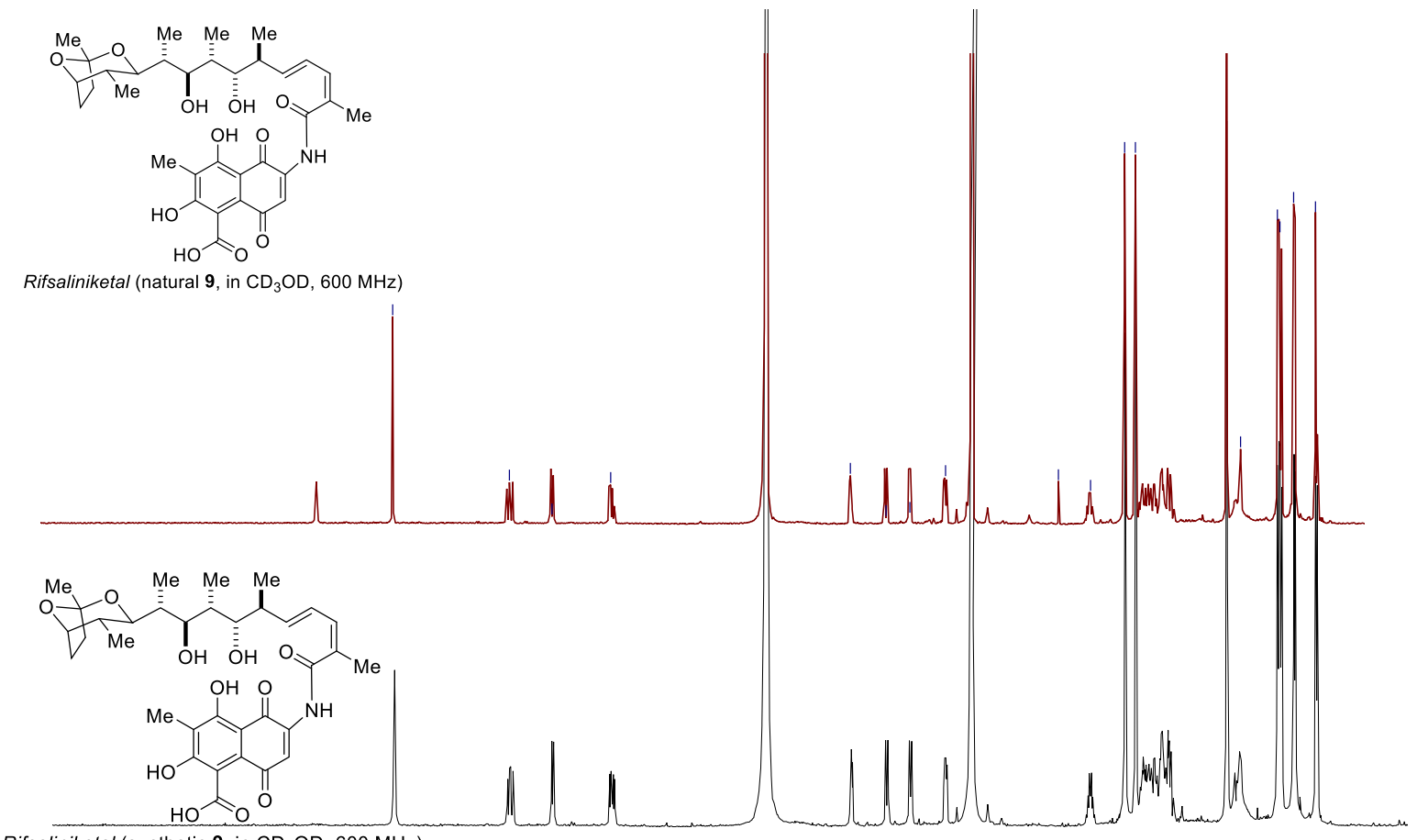

Rifsaliniketal (synthetic 9 , in $\mathrm{CD}_{3} \mathrm{OD}, 600 \mathrm{MHz}$ )
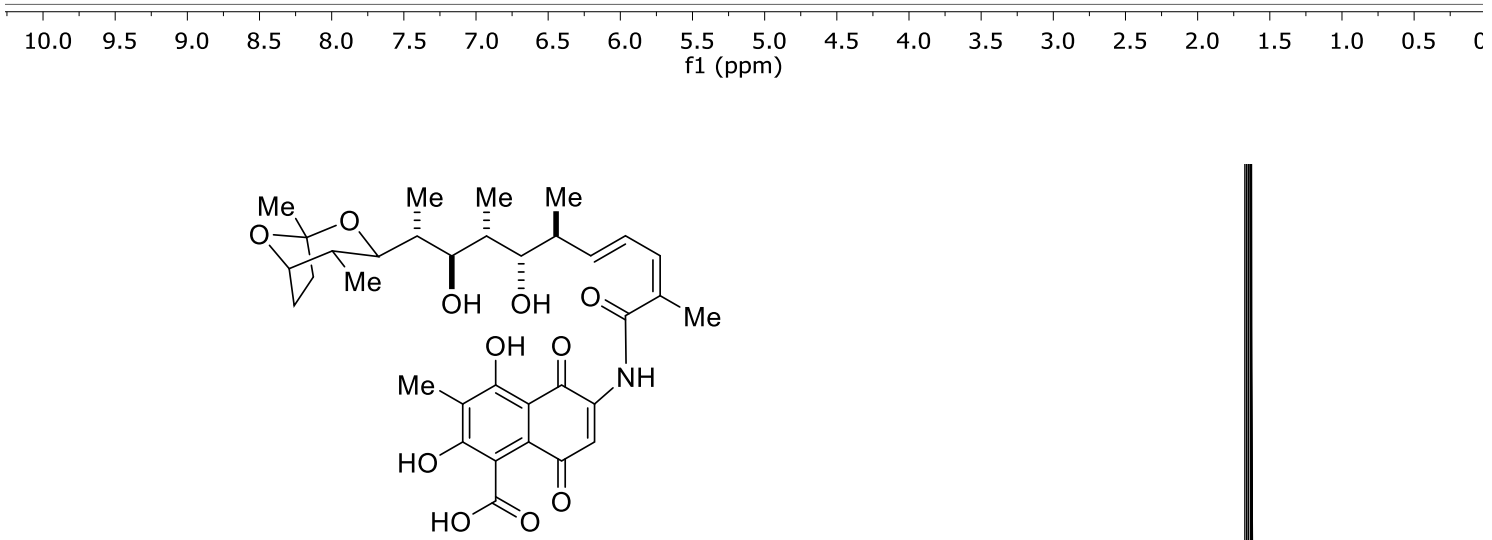

Rifsaliniketal (synthetic 9, in $\mathrm{CD}_{3} \mathrm{OD}, 150 \mathrm{MHz}$ )

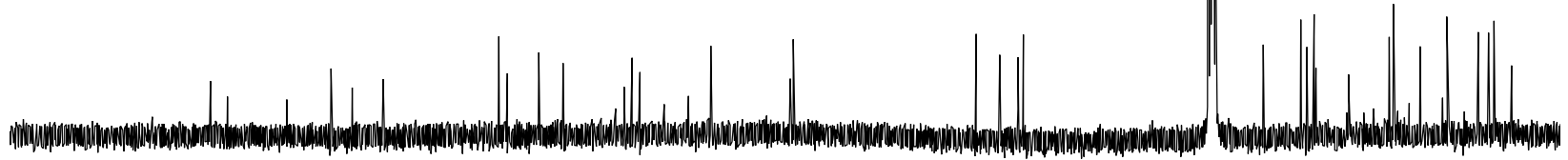

$\begin{array}{lllllllllllllllllllll}210 & 200 & 190 & 180 & 170 & 160 & 150 & 140 & 130 & 120 & \begin{array}{c}110 \\ \mathrm{f} 1(\mathrm{ppm})\end{array} & 100 & 90 & 80 & 70 & 60 & 50 & 40 & 30 & 20 & 10\end{array}$ 